\title{
5 Sakische Nekropolen mit Fürstenkurganen als Zentrale Orte der Nomaden
}

Im südöstlichen Siebenstromland ist eine enorm hohe Konzentration von früheisenzeitlichen Kurganen anzutreffen. Kennzeichen der Gräberfelder sind unterschiedlich lange Kurganketten. Die Größe der Tumuli ist unterschiedlich. Ihre Durchmesser variieren von einigen wenigen Metern bis zu über hundert Metern und es werden Höhen von einigen Zentimetern bis zu über zehn Metern erreicht. Die allergrößten Kurgane werden als Fürstenkurgane bezeichnet. Um die Gesamtheit der sakischen Gräberfelder zu differenzieren, wurden Nekropolen mit Fürstenkurgan und solche ohne unterschieden. Als große Kurgane gelten die Konstruktionen, die Durchmesser von mehr als 30 m und eine Höhe von über 2,0 m aufweisen. Falls Kurgane mit diesen Merkmalen festgestellt wurden, wurde das gesamte Gräberfeld dokumentiert und untersucht. Auf diese Weise wurden im gesamten Forschungsgebiet 28 Gräberfelder, die Großkurgane aufwiesen, sowie drei einzelne Kurgane ${ }^{\mathrm{I} 68}$ aufgenommen (Karte Io; vgl. Katalog, Einzelkurgane und Gräberfelder). Überwiegend handelt es sich jedoch um einfache Gräberfelder. In einem Fall grenzt ein solches Gräberfeld direkt an eine Siedlungsstelle ${ }^{\mathrm{I} 9}$. Die Kartierung der früheisenzeitlichen Nekropolen mit Fürstenkurganen im südöstlichen Siebenstromland zeigt, dass bei der Wahl des Platzes für ein Gräberfeld immer gleiche Landschaftsmarker ausschlaggebend waren. Es lassen sich zwei Verbreitungszonen unterscheiden (Karte II).

Die erste Verbreitungszone befindet sich an der nördlichen Seite des Tien-Shan, und zwar dort wo das nördliche Lösshügelland des Transili-Alatau in die Schwemmfächer der einzelnen Flüsse übergeht. Die Nekropolen sind in der Regel auf Schwemmfächern (800-900 m ü NN) angelegt und vom Lösshügelland im Durchschnitt I,5-3, o km entfernt. Diese Zone hat eine Ost-West Ausdehnung von I33 km, die vom unteren Flusslauf des Čilik und dem mittleren Flusslauf des Bol'šaja Almatinkar7o begrenzt wird. Die Nord-Süd Ausdehnung beträgt dagegen nur 16 km7. Es handelt sich also um einen schmalen, aber langgezogenen Landschaftsstreifen an der nördlichen Seite des Transili-Alatau, der für die Errichtung der Gräberfelder mit großen Fürstenkurganen benutzt wurde.

Eine zweite Konzentration dieser früheisenzeitlichen Bodendenkmäler wurde auf dem Hochplateau Kegen (I853-2055 m ü NN) und jenseits des Kuluktau-Gebirges (Nordseite) festgestellt. Im Gegensatz zur ersten Verbreitungszone wurden hier die Gräberfelder sowohl im mittleren Bereich des $42 \times$ $35 \mathrm{~km}$ großen Plateaus, als auch in der Peripherie des Plateaus, im Lösshügelland, festgestellt. Allerdings ist anzumerken, dass nicht die gesamte Peripherie des Plateaus mit solchen großen Kurganen der Früheisenzeit bebaut wurde. Gräberfelder mit Fürstenkurganen finden sich nur im Lösshügelland in der Nordhälfte des Plateaus, nämlich auf den Riedelkanten. Hier bilden sie eine Art peripheren Bogen, der im Temirlik Gebirge, das im Nordosten des Plateaus liegt, beginnt und sich über das im Norden gelegene Kuluktau-Gebirge nach Westen bis zum südlichen Ausläufer des Čaryn-Canyon ausdehnt.

In den Bergtälern des Transili-Alatau und an dessen Südseite treten solche Nekropolen gar nicht auf. Im Čilik Tal, an der Nordseite des Kungej-Alatau, befinden sich einzelne Kurgane (Karte II, Nr. 42,

I68 Zwei Kurgane 400 m westlich vom Dorf Žalauly und ein Kurgan $5 \mathrm{~km}$ südwestlich vom Dorf Žalauly.

I69 Archäologischer Komplex Kiikpaj; Vgl. Katalog.

I70 Vom Gräberfeld Žoan Tobe im Osten bis zur Nekropole

Ulžan im Westen (siehe Karte II, Nr. 27 und Nr. I).
I7I Die maximalen Entfernungen wiesen die Gf. Žoan Tobe im Norden und Gf. Asy Saga im Süden auf (siehe Karte II, Nr. 27 und Nr. 29). 
43, 45). Die Täler Žalanaš und Sogetin im Osten des Forschungsgebietes sind vom Torajgyr Gebirge und der Bergkette Sarytau umgeben. In diesen Tälern konnten keine Gräberfelder mit Fürstenkurganen festgestellt werden.

\subsection{Die Bauweise der Kurgane}

Die großen Fürstenkurgane der Früheisenzeit im südöstlichen Siebenstromland stellen überwiegend abgerundete, plattformähnliche Konstruktionen mit einer abgeflachten Kuppe dar. Die Kurgane haben drei steile und einen sanften Abhang (Abb. 27). In einigen Fällen (I6 Kurgane von zehn unterschiedlichen Gräberfeldern) wiesen die Tumuli eine viereckige, pyramidenähnliche Form mit einer abgeflachten Kuppe auf ${ }^{\mathrm{i} 72}$ (Abb. 28).

Insgesamt wurden 3I Fundorte mit Großkurganen der sakischen Elite im Arbeitsgebiet dokumentiert. Desweiteren wurde eine Siedlung mit zugehöriger Nekropole, die nur aus Kleinkurganen besteht, festgestellt. Die Gesamtzahl der Grabanlagen beläuft sich auf 955 Kurgane (Tabelle I2), von denen 3I9 als Großkurgane bezeichnet werden können. Fast alle Hügel zeigen Beraubungsspuren wie vertikal angelegte Raubtrichter oder horizontal hinein gegrabene Raubschächte.

\subsubsection{Groß- und Kleinkurgane}

Bei der Betrachtung der Kurgangröße schlug zuerst K. A. Akišev folgende Differenzierung der sakischen Kurgane für das Ili-Gebiet vor: a) große Kurgane (8-I7 m) - Königs- bzw. Fürstenkurgane; b) mittelgroße Kurgane (5-6 m) - Kurgane des Adels; c) Kleinkurgane (I-2 m) - Kurgane der Helden (Akišev/ Kušaev I963, 86). Später überarbeitete der Forscher seine Unterteilung, indem er die genauen Maßangaben entfernte und die soziale Kategorisierung der Bestatteten veränderte. Demzufolge schlug er drei Größenkategorien vor: a) große Kurgane - Königs- bzw. Fürstenkurgane; b) mittelgroße Kurgane - Kurgane des Adels und der Helden; c) Kleinkurgane - Kurgane der Krieger (Akišev K. A. I978, 56). Einen ähnlichen Vorschlag, jedoch unter Berücksichtigung der Beigaben, machte A. G. Grač im Zuge der Analyse der Kurgane des Altaj und Tuvas (Grač I975, I58-I74). Für B. N. Mozolevskij war die Aussage von Herodot gewichtig, dass jeder Teilnehmer an der Bestattungszeremonie bei der Errichtung der Kurganaufschüttung mitwirkte und bemüht war, einen möglichst großen Hügel aufzubauen (Herodot [4-7I], 305). Denn die Kurgangröße spiegelte die Stellung der bestatteten Person innerhalb der Gemeinschaft wider. Allerdings wurde bei Untersuchungen relativ häufig festgestellt, dass die Kurgangröße nicht immer im Verhältnis zur Qualität der Grabausstattung steht. Daher behauptete der Forscher, dass die bloße Kurgangröße ein relatives Merkmal sei und allein keinen realen Status des Bestatteten anzeige (Mozolevs'kij I979, I56; vgl. Mozolevskij/Polin 2005, 299). Später veröffentlichten B. N. Mozolevskij und S. V. Polin anhand ihrer Forschungen im mittleren Dnepr-Gebiett73 eine Größengliederung für die Kurgane des nordpontischen Raumes, die aus vier Gruppen besteht: I. Gruppe - Kurgane-Giganten, die

I72 Siehe Katalog: ein Kurgan 5 km südwestlich vom Dorf Žalauly, Gf. Asy Saga, K3; Gf. am Stausee Bartogaj, K6; Gf. Bes Tobe, K2; Gf. Kyzylšaryk, K2; Gf. Novoalekseevka, K4 und Kio sowie Kleinkurgane 2, 3 und 7, die I956 von I. I. Kopylov (Gf. Novoalekseevka, Forschungs- geschichte) ausgegraben worden sind; Gf. Taučilik, 6 KI; Gf. Turgen, Kı2; Gf. Žoan Tobe, K2, K6, K8 und Gf. Žylysaj-I, K6.

I73 Im „Skythischen Herros“. 


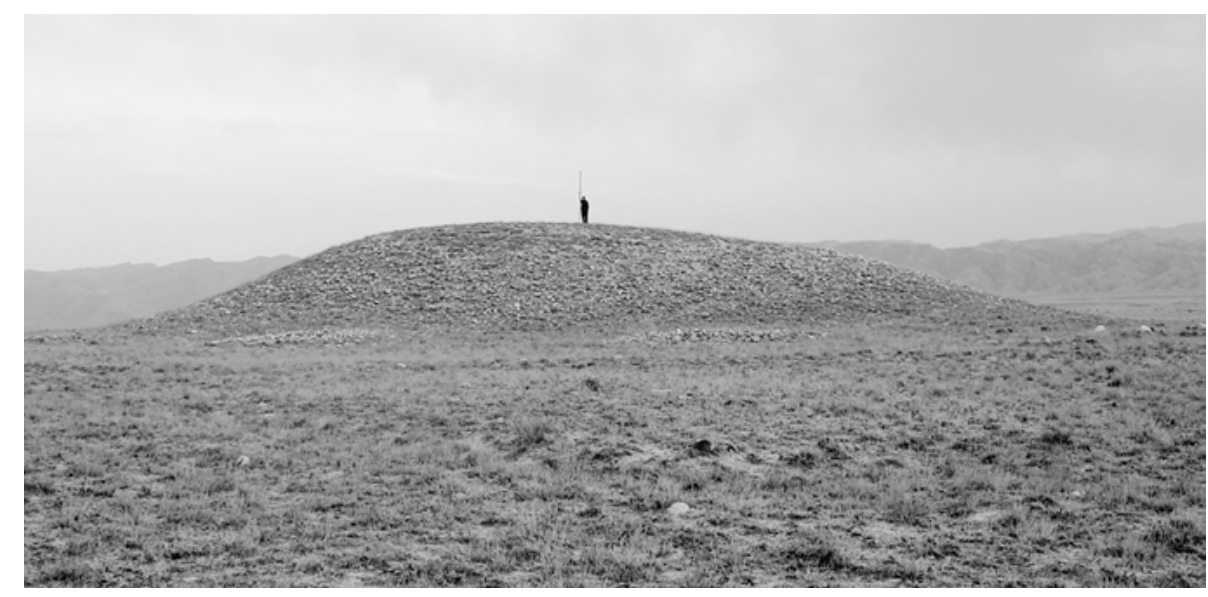

Abb. 27 | Gräberfeld Asy Saga. Kurgan 4. Blick von WNW

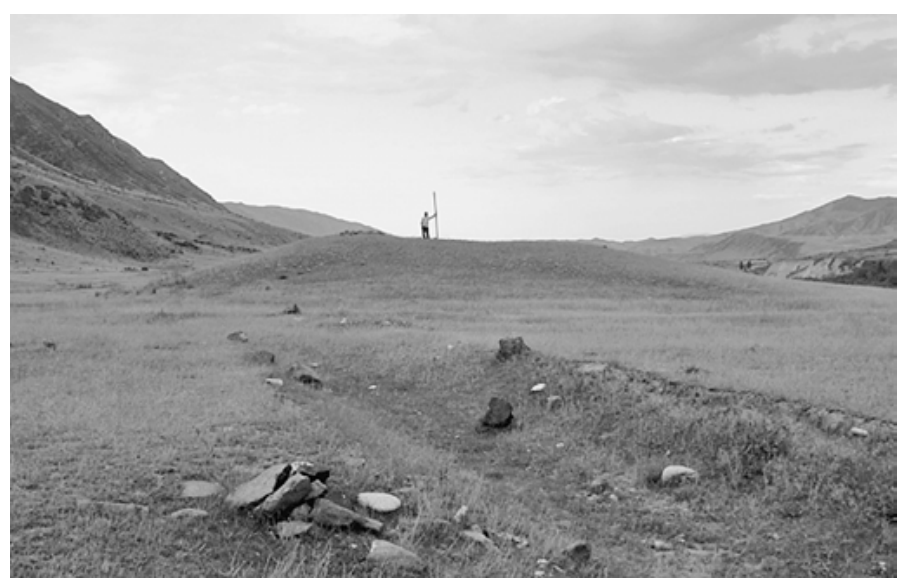

Abb. 28 | Viereckiger Kurgan. Gräberfeld Taučilik-6. Kurgan I. Blick von W

mit einer Höhe von I4-22 m für die Hauptkönige, Basileia, errichtet worden waren; II. Gruppe - Kurgane mit einer Höhe von 8-II m, die für die Familienmitglieder des Hauptkönigs und für die Nebenkönige, ebenso Basileia, gebaut worden waren; III. Gruppe - Kurgane mit einer Höhe von 4,8-7,0 m, die für den Nomarchen (Häuptlingen) der Nomoi (administrative Unterabteilungen) gedacht waren; und IV. Gruppe - 2,0-4,5 $\mathrm{m}$ hohe Kurgane, die für den Stammesältesten und für hoch angesehene Stammesmitglieder errichtet worden waren (Mozolevskij/Polin 2005, 299-300; vgl. Parzinger 2004, 90).

Die statistische Auswertung der Verteilung der Kurgane bezüglich der Parameter „Durchmesser“ und „Höhe“ zueinander mittels eines Streudiagramms (Abb. 29) zeigt, dass bei Betrachtung aller untersuchten Gräberfelder mit großen fürstlichen Kurganen nur zwei Cluster entstehen ${ }^{174}$. Die Punktwolken des Streudiagramms zeigen zwei Gruppen von Kurgangrößen: I. Gruppe - Kurgane mit einer 
Tabelle 12 | Merkmale der früheisenzeitlichen Kurgane (in alphabetischer Ordnung)

\begin{tabular}{|c|c|c|c|c|c|c|c|c|c|c|c|}
\hline \multirow{2}{*}{ 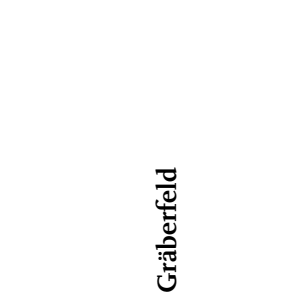 } & \multirow[t]{2}{*}{ 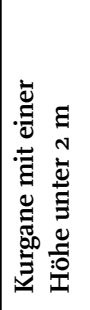 } & 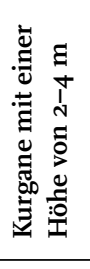 & 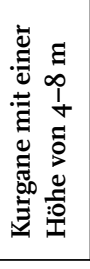 & 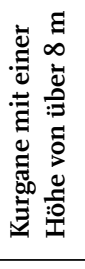 & \multirow[t]{2}{*}{ 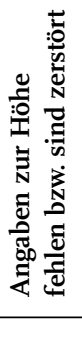 } & \multirow[t]{2}{*}{ 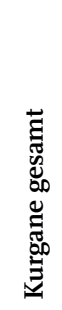 } & \multirow[t]{2}{*}{ 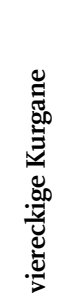 } & \multirow[t]{2}{*}{ 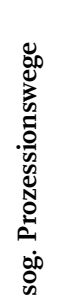 } & \multirow{2}{*}{ 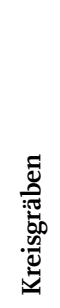 } & \multirow{2}{*}{ 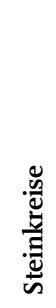 } & \multirow{2}{*}{ 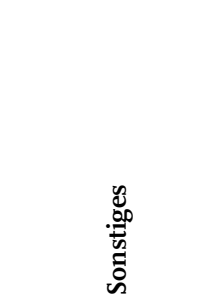 } \\
\hline & & \multicolumn{3}{|c|}{ Großkurgane } & & & & & & & \\
\hline${ }_{1} \mathrm{~K} 5 \mathrm{~km}$ SW von Žalauly & & I & & & & I & I & & I & & \\
\hline $2 \mathrm{~K} 400 \mathrm{~m} W$ von Žalauly & & I & I & & & 2 & & & & & \\
\hline${ }_{3} K{ }_{7} \mathrm{~km}$ W von Saty & & 2 & I & & & 3 & & & & & \\
\hline Aksaj-9 & 28 & 2 & & & & 30 & & I & & 3 & \\
\hline$A k s ̌ o k \gamma-4$ & 4 & 9 & I & & & I4 & & & & & \\
\hline Aktasty-3 & $4 \mathrm{I}$ & 2 & & & & 43 & & I & & 6 & \\
\hline Asy Saga & & 2 & 3 & I & & 6 & I & 3 & & & „Vorsprung“ IX \\
\hline Bartogaj Stausee & 39 & & & & & 39 & I & I & & 2 & \\
\hline Bes Tobe & 32 & $\mathrm{I} 2$ & 2 & & I & 47 & I & & & & \\
\hline Bestobe-3 & & 6 & & & & 6 & & & & & \\
\hline Boroldaj & 26 & I4 & 9 & 2 & I & 52 & & & I8 & 3 & Erdwall 8x \\
\hline Ereul & I & 16 & I8 & & & 35 & & & I & I & \\
\hline Issyk & 26 & 2I & I7 & 3 & I6 & 83 & & & & I & \\
\hline Plateau Kegen & 2 & 20 & 7 & 8 & & 37 & & & I & & $\begin{array}{l}\text { Plattform Ix und } \\
\text { Baurampen Ix }\end{array}$ \\
\hline Komplex Kiikpaj & I2 & 3 & & & & I5 & & & & & \\
\hline Kokpijaz & 4 & 3 & & & & 7 & & & & & \\
\hline Krasnyj Vostok & & 2 & & & & 2 & & & & & \\
\hline Kyzylšaryk & I6 & 7 & IO & & 4 & 37 & I & 2 & & & \\
\hline Nordwestlicher Friedhof & 5 & & 3 & I & & 9 & & & & & \\
\hline Novoalekseevka & 8 & 3 & 8 & I & I & $2 \mathrm{I}$ & 5 & & & & \\
\hline Komplex Sarytau & IO3 & & & & & I03 & & & & & \\
\hline Sarytau „A“ & & 3 & & & & 3 & & & & & \\
\hline Saty & 22 & 3 & & & & 25 & & & & & \\
\hline Šyrganak-2 & & 3 & 4 & $\mathrm{I}$ & & 8 & & & & & \\
\hline Taučilik-6 & I44 & & 2 & & & 146 & I & & & & \\
\hline Togyzbulak & I & 5 & & I & & 7 & & & I & & \\
\hline Turgen & 2 & 8 & 23 & 2 & I & 36 & I & 2 & & 2 & Steinwall Ix \\
\hline Ulžan & & 2 & 3 & & & 5 & & & & & Erdwall Ix \\
\hline Žalauly-2 & $5 \mathrm{I}$ & I4 & 3 & & & 68 & & I & & 6 & \\
\hline Žalauly-8 & II & 5 & & & & I6 & & & 6 & & $\begin{array}{l}\text { Doppelter Stein- } \\
\text { kreis ohne Kurgan }\end{array}$ \\
\hline Žoan Tobe & 22 & 4 & $\mathrm{I}$ & $\mathrm{I}$ & 3 & $3 \mathrm{I}$ & 3 & I & & & \\
\hline Žylysaj-1 & 9 & 9 & & & & I8 & I & 7 & & I & \\
\hline Insgesamt: & 609 & I82 & II6 & $2 \mathrm{I}$ & 27 & 955 & 16 & I9 & 28 & 23 & \\
\hline
\end{tabular}


Höhe von o,I $\mathrm{m}$ bis zu $8 \mathrm{~m}$ und einem Durchmesser von $3 \mathrm{~m}$ bis zu $80 \mathrm{~m}$; und die 2. Gruppe - eine Gruppe von Kurganen, die die Maße der I. Gruppe übertrifft.

Um die Großkurgane anhand ihrer Größe voneinander zu unterscheiden, steht die Frage im Mittelpunkt, welche Parameter dabei am aussagekräftigsten sind. Das Verhältnis der äußeren Eigenschaften eines Kurgans von Durchmesser zur Höhe (Abb. 29) erwies sich zunächst als nicht aussagekräftig. Bei der Häufigkeitsanalyse der einzelnen Parameter zeigte sich, dass das Auftretten unterschiedlicher Kurgane mit einem bestimmten Durchmesser im gesamten Untersuchungsgebiet als ein charakteristisches Merkmal nicht aussagekräftig ist (Abb. 30), da zu viele, unübersehbare Kombinationsmöglichkeiten entstanden.

Dagegen ist die Analyse der Häufigkeit von Kurganen mit einer bestimmten Höhe (Abb. 3I) etwas überschaubarer. Die hier entstandenen Cluster erlauben in gewisser Weise verschiedene Höhenkategorien der Tumuli herauszuarbeiten. Jedoch scheint diese Gliederung auch nicht ganz eindeutig zu sein.

Um die sakischen Kurgane, vor allem jedoch die Großkurgane im südöstlichen Siebenstromland, trotzdem voneinander zu unterscheiden, ist man auf eine „künstliche“ Differenzierung anhand des Ausmaßes der Grabhügel angewiesen. Damit ergibt sich die Möglichkeit, für einige bestimmte Fundstellen architektonische Regeln zu definieren, wie z.B. die Größe der Anlagen. Allerdings scheint es hier nicht uneingeschränkt sinnvoll zu sein, lediglich mit der Größe eines Kurgans auch einen bestimmten sozialen Status des bestatteten Individuums zu verbinden, ohne jedoch eine Analyse des ge-

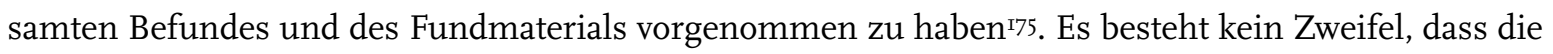
großen Kurgane für besondere Personen gebaut worden sind. Allerdings bleibt die Frage nach dem genauen Status des Bestatteten innerhalb der Gesellschaft weiterhin offen. So zum Beispiel wurde der "Goldene Mann“ von Issyk in einer Nebenkammer eines 6 m hohen Grabhügels entdeckt (vgl. Katalog, Forschungsgeschichte des Gräberfeldes Issyk). Die Tatsache, dass das bestattete Individuum in einer Nebenkammer lag, könnte ein Indiz für seine geringere Bedeutung sein. Andererseits zeigten die Untersuchungen am Fürstenkurgan von Aržan 2 in Tuva (Russische Föderation), dass die Hauptbestattung nicht immer exakt in der Mitte eines Kurgans angelegt sein muss (vgl. Čugunov u.a. 20I0, I6-22). Außerdem ist der Kurgan des „Goldenen Mannes“ mit einer Höhe von 6 m nicht der größte Kurgan der Nekropole, aber im Vergleich zu einer durchschnittlichen Kurganhöhe von $4 \mathrm{~m}$ etwas höher. Einige Kurgane des Gräberfeldes Issyk weisen dagegen eine Höhe von 9-Io $\mathrm{m}$ auf ${ }^{\mathrm{I} 76}$. So bleibt bezüglich dieses Merkmales der tatsächliche soziale Status des „Goldenen Mannes“ unklar. Weiterhin stellt sich die Frage, ob die besondere Stellung, die ja durch den Bau des Grabmals durch die Gemeinschaft hervorgehoben werden sollte, tatsächlich auf eine eigene Leistung des Verstorbenen zurückzuführen ist, zumal der „Goldene Mann“ von Issyk bereits im jugendlichen Alter verstarb (I6-I8 Jahre nach Akišev K. A. I978, 62; vgl. Parzinger 2006, 660). Es ist eher anzunehmen, dass das hohe soziale Ansehen des "Goldenen Mannes“ auf seine Abstammung zurückzuführen ist.

Setzt man die Höhenkategorien in Bezug zu Studien über die Größengliederung der früheisenzeitlichen Großkurgane im skytho-sakischen Kulturraum (Akišev/Kušaev I963, 86; Akišev K. A. I978, 56; Grač 1975, I58-I74; Mozolevskij/Polin 2005, 299-300), schlägt der Verfasser vor, die Großkurgane anhand ihrer Größe in drei „künstliche“ Kategorien zu unterteilen: a) große Kurgane - Höhen von 2 bis zu 4 m; b) größere Kurgane mit einer Höhe von 4 bis 8 m; und c) sehr große Kurgane - mit einer Höhe über $8 \mathrm{~m}$.

I75

An dieser Stelle möchte ich der Meinung Mozolevskijs absolut zustimmen (Mozolevs'kij 1979, 156).
I76 Vgl. Katalog, Gf. Issyk, Kurgancharakteristik: KI, K2 und $\mathrm{K} 3$. 


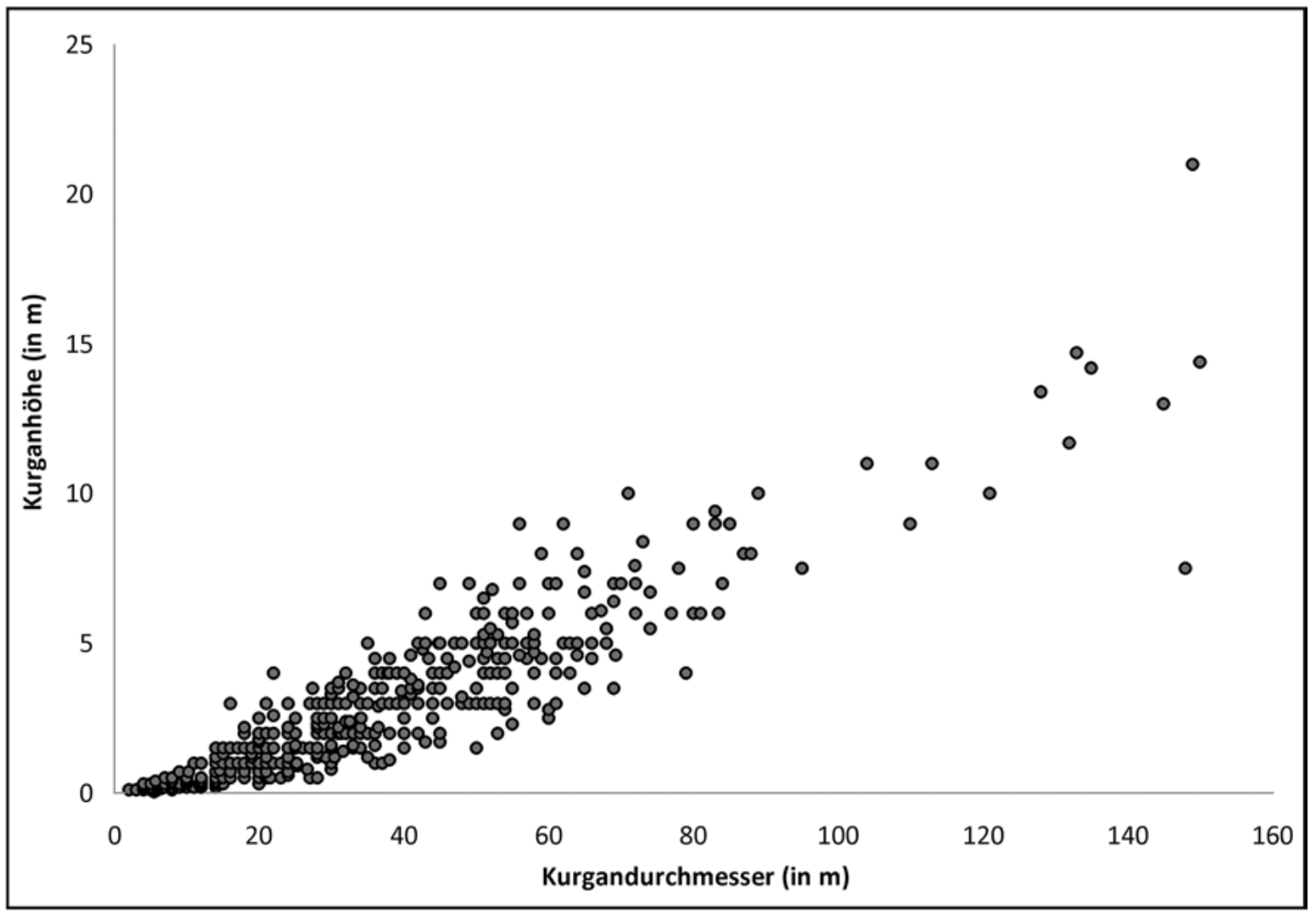

Abb. 29 | Statistische Verteilung der Kurgane bezüglich ihrer Größe mit der Berücksichtigung des Durchmessers und der Höhe

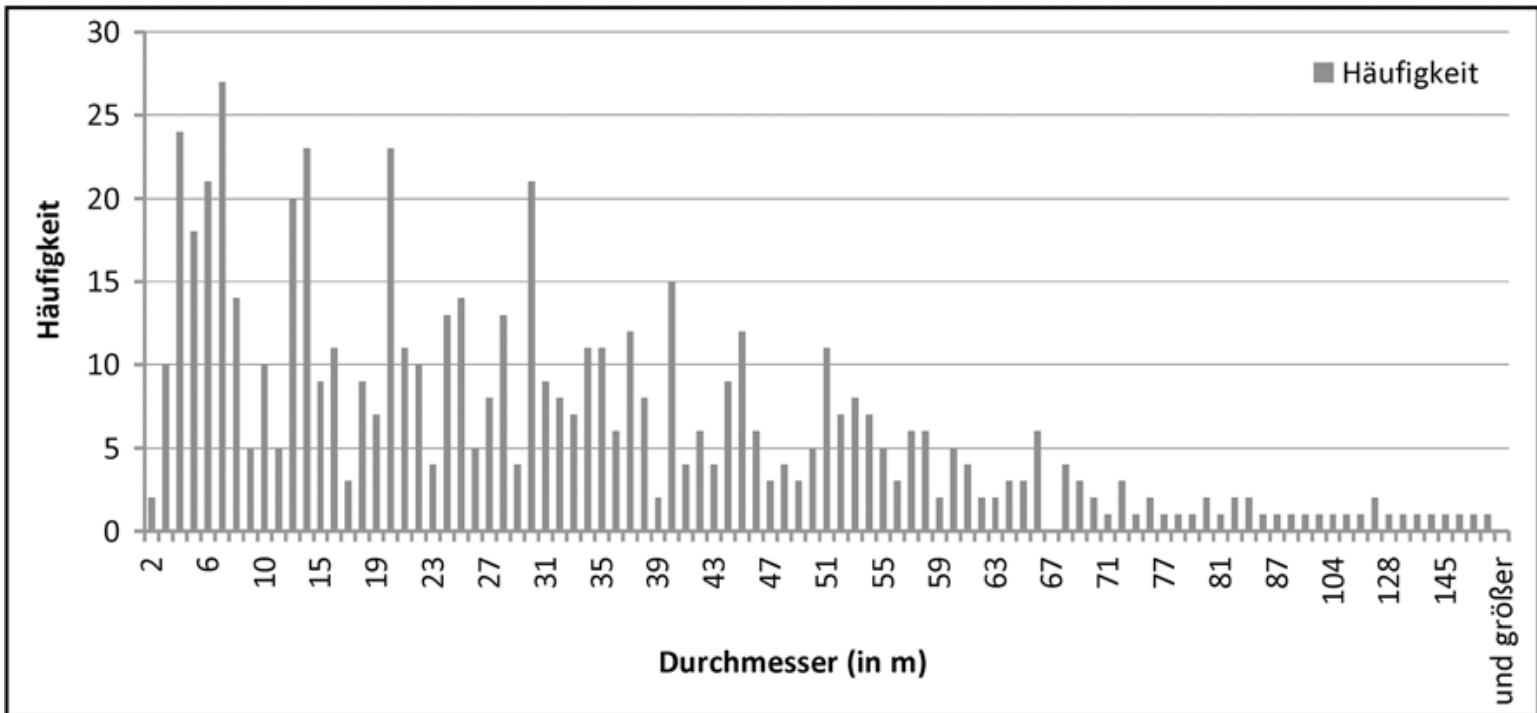

Abb. 30 | Häufigkeit von Kurganen mit einem bestimmten Durchmesser 


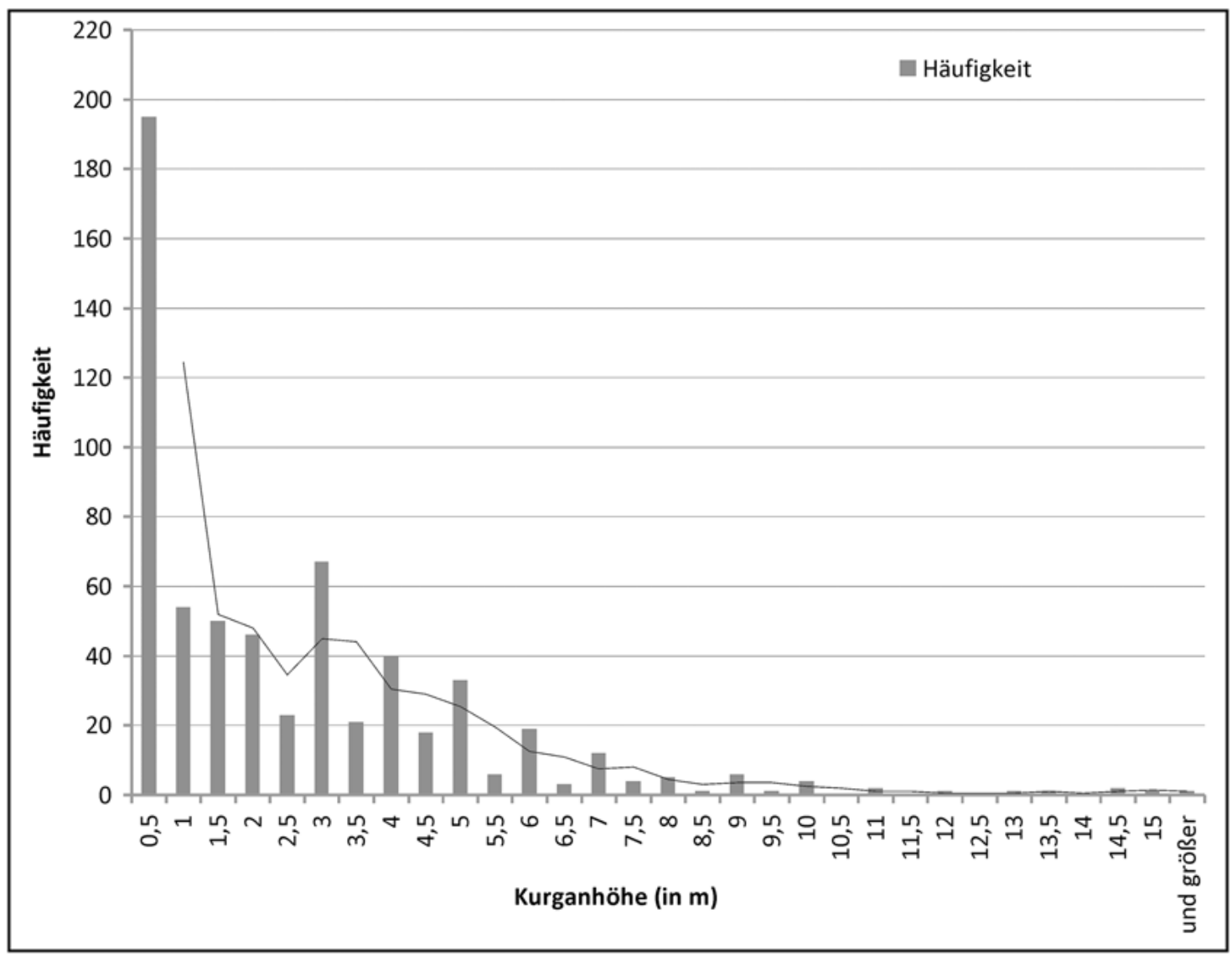

Abb. 31 | Häufigkeit von Kurganen mit einer bestimmten Höhe

Auf diese Weise bestehen die Gräberfelder aus jeweils zwei Kurgangruppen - Kleinkurgane (bis zu 2 m Höhe) und Großkurgane (über 2 m Höhe), die wiederum nach der Größe drei Unterkategorien bilden (Abb. 32). Die statistische Verteilung der Kurgane nach dem diagnostischen Merkmal ihrer Größe erbrachte das in Abb. 33 dargestellte Ergebnis (vgl. Abb. 29).

In Anbetracht der oben erwähnten Tatsachen wird vorgeschlagen, zunächst lediglich auf eine einfache Differenzierung nach Größe der Kurgane einzugehen. Die sich daraus ergebende vermeintliche soziale Gliederung, kann nur allgemein bestätigt werden ${ }^{177}$, nämlich dass die in besonders großen Kur-

Im Bezug auf ähnliche, bereits untersuchte „skythische“ Großkurgane Eurasiens (Rudenko I95I; Mozolevs'kij I979; Grjaznov 1984; Alekseev u.a. I99I; Galanina I997; Itina/Jablonskij I997; Polos'mak 200I; Mozolevskij/Polin 2005; Čugunov u.a. 2010; Parzinger u.a. 2010) kann man vermuten, dass die sehr großen Kurgane (über $8 \mathrm{~m}$ Höhe) für die sakischen Könige bzw. Fürsten, die größeren Kurgane $(4-8 \mathrm{~m}$ ) für den Adel (Nomarchen) und die Helden und die großen Kurgane (2-4 m) für den ruhmreichen Krieger, für den Stammesältesten und besonders angesehene Gesellschaftsmitglieder errichtet worden sind. Betrachtet man die früheisenzeit- lichen Gräberfelder jedoch als Friedhöfe einer Dynastie von Führungseliten bzw. einer Führungseinheit (Gass 20Iıа, 68), so könnte die unterschiedliche Größe der Kurgane auf ein steigendes oder sinkendes Ansehen des jeweiligen Mitgliedes der Dynastie hindeuten. Solange jedoch keine einzige Kurgankette mit Großkurganen der Saken im Forschungsgebiet vollständig ausgegraben und untersuch ist, müssen die Überlegungen in Bezug auf das Verhältnis von Kurgangröße zu sozialem Status der bestatteten Individuen sowie eine mögliche Verwandtschaft zwischen den hauptbestatteten Personen in den Kurganen einer Kette Theorie bleiben. 


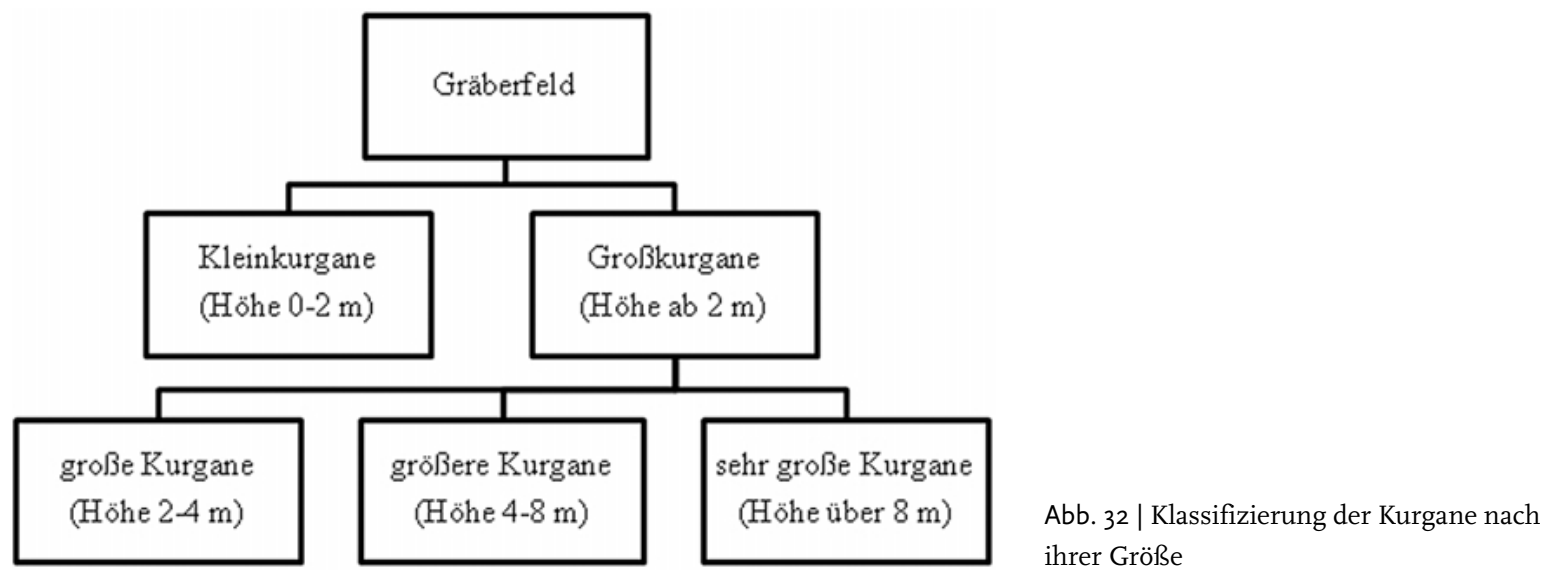

ganen Bestatteten ein hohes soziales Ansehen hatten. Eine weiterführende soziale Gliederung scheint jedoch voreilig zu sein.

Insgesamt dominieren unter den Kurganen die $6 \circ 9$ Kleinkurgane ( $64 \%$ aller untersuchten früheisenzeitlichen Kurgane). Zu knapp 3\% der Kurgane (27 Grabhügel) konnten aufgrund des hohen Zerstörungsgrades keine Aussagen bezüglich der Größe getroffen werden. Die Kategorie der „großen Kurgane“ (2-4 m) stellt die zahlenstärkste unter den Großkurganen dar (I82 Konstruktionen, I9\%). Es folgen „größere Kurgane“ (4-8 m) mit einer Gesamtzahl von II6 Grabhügeln (I2 \%). Am seltensten wurden „sehr große Kurgane“ (über $8 \mathrm{~m}$ ) für Personen, die das höchste Ansehen der Gesellschaft genossen, errichtet (2I Konstruktionen, 2\%).

Vergleicht man diese Daten zwischen den Verbreitungszonen der Gräberfelder mit großen fürstlichen Kurganen, so fällt auf, dass eine ähnliche Situation (Abb. 34) lediglich in der zweiten, südöstlich gelegenen Verbreitungszone (Karte II) existiert ${ }^{17}$. Ein anderes Bild zeichnet sich für die Großkurgane in der ersten Verbreitungszone ${ }^{\mathrm{I79}}$ (Karte II) entlang der Nordseite des Transili-Alatau (Abb. 35) ab. Hier kommen 79 größere Kurgane (4-8 m) vor, also $26 \%$ aller untersuchten Anlagen, sowie 75 große Kurgane (2-4 m), was einen Anteil von 25\% ausmacht. Die Häufigkeit dieser beiden Kurgankategorien ist also fast gleich. Das gilt nicht für die zweite Verbreitungszone. Hier treten die großen Kurgane (2-4 m; I02 Konstruktionen, 29\%) wesentlich häufiger auf als die größeren Kurgane (4-8 m; 34 Konstruktionen, I०\%). Sehr große Kurgane wurden in beiden Verbreitungszonen selten festgestellt, nämlich elf Anlagen (4\%) in der ersten und zehn Grabhügel (3\%) in der zweiten Zoner8o.

Im Durchschnitt unterscheiden sich die Maße der Großkurgane beider Verbreitungszonen nur um einige Meter (Tabelle I3). Daher kann die sich ergebende Differenz als irrelevant betrachtet werden.

I78 Da die Nekropole des archäologischen Komplexes Sarytau nur aus kleinen, nicht fürstlichen Kurganen besteht, wurden diese Kurgane (insgesamt I03) hier nicht berücksichtigt und von den statistischen Analysen ausgeschlossen.

I79 Da bei 27 Kurganen der ersten Verbreitungszone aufgrund des hohen Zerstörungsgrades keine Aussagen bezüglich ihrer Größe gemacht werden können, wurden sie zwar berücksichtigt, jedoch von den statistischen Analysen ausgeschlossen.

I80 Von insgesamt 329 Kurganen der ersten Verbreitungszone konnten lediglich 302 in die Analyse einbezogen werden, da die restlichen einen zu schlechten Erhaltungszustand aufwiesen. Von den 349 Grabhügeln der zweiten Verbreitungszone wurden alle ausgewertet. Im Raum zwischen den Zonen, im Čilik-Tal, an der Nordseite des Kungej-Alatau befinden sich I74 weitere in die Untersuchung einfließende Kurgane. Davon konnten I66 Kurgane als Klein- (die Durchschnittliche Höhe solcher Kurgane ist 0,7 m) und nur acht als Großkurgane bezeichnet werden. Von diesen acht Kurganen wiesen fünf eine Größe von 2-4 $\mathrm{m}$ und drei von 4-8 $\mathrm{m}$ auf. Sehr große Kurgane treten in diesem Gebiet gar nicht auf. 


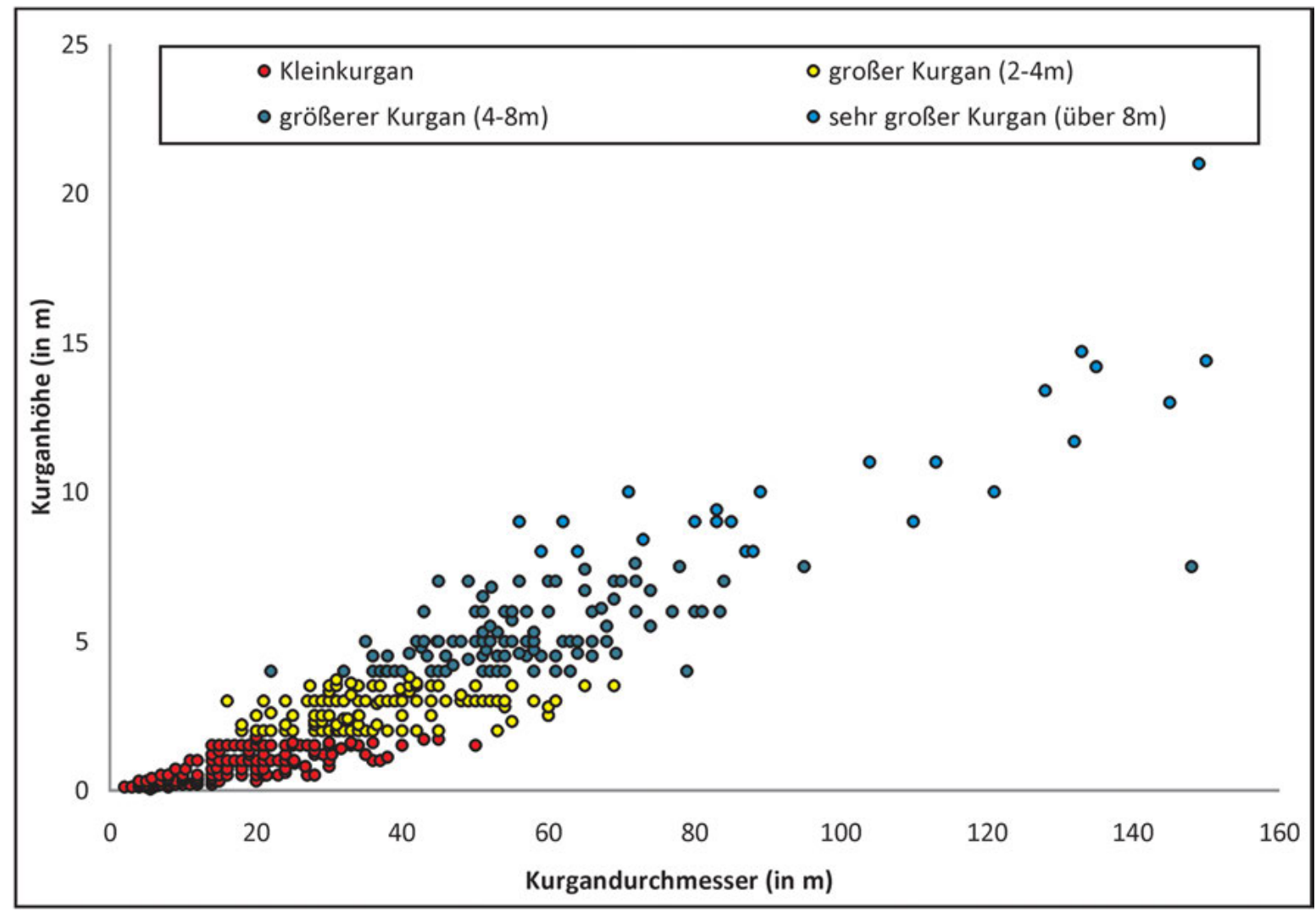

Abb. 33 | Statistische Verteilung der Kurgane bezüglich ihrer Größe unter Berücksichtigung des Durchmessers und der Höhe (farbig nach den künstlich definierten Kategorien gegliedert)

Abb. 34 | Verhältnis der Kurgane bezüglich ihrer Größe in der zweiten Verbreitungszone

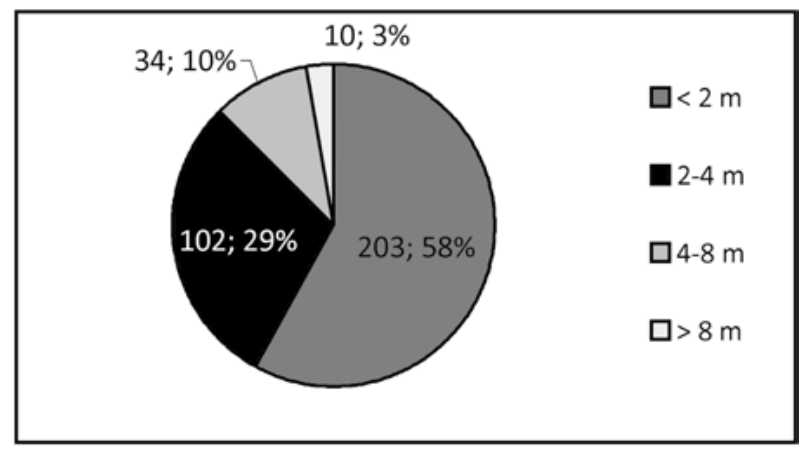

Abb. 35 | Verhältnis der Kurgane bezüglich ihrer Größe in

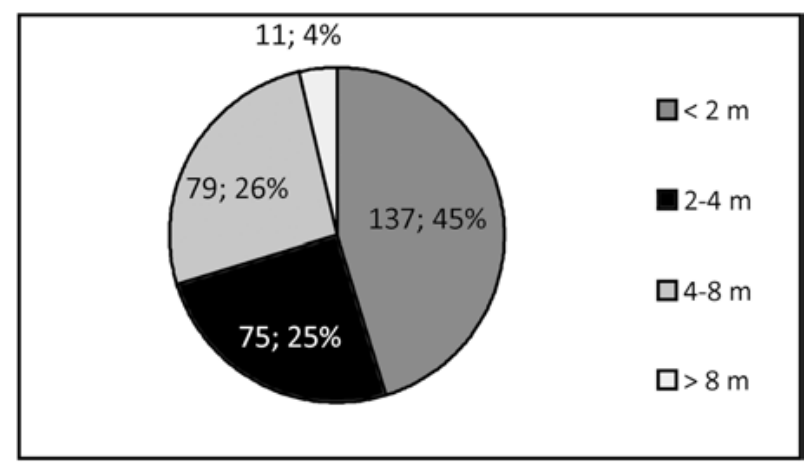
der ersten Verbreitungszone 
Tabelle 13 | Größenverhältnisse innerhalb der Verbreitungszonen

\begin{tabular}{|c|c|c|c|c|}
\hline \multirow{2}{*}{ Größe der Kurgane } & \multicolumn{2}{|c|}{ Erste Verbreitungszone } & \multicolumn{2}{c|}{ Zweite Verbreitungszone } \\
\cline { 2 - 5 } & $\begin{array}{c}\text { Durchschnittliche } \\
\text { Höhe }\end{array}$ & $\begin{array}{c}\text { Durchschnittlicher } \\
\text { Durchmesser }\end{array}$ & $\begin{array}{c}\text { Durchschnittliche } \\
\text { Höhe }\end{array}$ & $\begin{array}{c}\text { Durchschnittlicher } \\
\text { Durchmesser }\end{array}$ \\
\hline $2-4 \mathrm{~m}$ & $3, \mathrm{Im}$ & $40 \mathrm{~m}$ & $2,8 \mathrm{~m}$ & $35 \mathrm{~m}$ \\
\hline $4-8 \mathrm{~m}$ & $5,6 \mathrm{~m}$ & $60 \mathrm{~m}$ & $5,6 \mathrm{~m}$ & $52 \mathrm{~m}$ \\
\hline$>8 \mathrm{~m}$ & $\mathrm{II}, 6 \mathrm{~m}$ & $105 \mathrm{~m}$ & $\mathrm{I} 2 \mathrm{~m}$ & $103 \mathrm{~m}$ \\
\hline
\end{tabular}

Da die meisten untersuchten Nekropolen nicht nur aus großen, sondern auch aus kleinen Kurganen bestehen, kann man sie in vier Gruppen gliedern: I. Gräberfelder, die nur aus Großkurganen bestehen; II. Nekropolen, wo Großkurgane mehr als die Hälfte aller Kurgane ausmachen; III. Gräberfelder, die zu gleichen Teilen aus Groß- und Kleinkurganen bestehen; und IV. Nekropolen die überwiegend aus Kleinkurganen bestehen, jedoch auch einige Großkurgane aufweisen. Die graphische Darstellung (Abb. 36) zeigtr $^{18 I}$, dass im südöstlichen Siebenstromland am häufigsten entweder Nekropolen vorkommen, in denen sich ausschließlich Großkurgane befinden (neun Gräberfelder, $30 \%$ aller untersuchten Nekropolen mit Großkurganen) oder Bestattungsplätze, die überwiegend Kleinkurgane und einen geringen Anteil von Großkurganen aufweisen (elf Gräberfelder, 37\%). Sehr selten sind Nekropolen, in denen die Zahl der Groß- und der Kleinkurgane gleich ist. Bei 23\% der Fälle (sieben Gräberfelder) bilden die Großkurgane eine Mehrheit gegenüber den übrigen Anlagen. Es sei jedoch angemerkt, dass diese Situation lediglich einen gegenwärtigen Forschungsstand widerspiegelt. Fast alle Gräberfelder befinden sich auf modernen Ackerflächen und es muss die Möglichkeit der völligen Zerstörung, vor allem der Kleinkurgane, durch die landwirtschaftlichen Aktivitäten der letzten 50-150 Jahre ${ }^{\mathrm{I} 2}$ in Betracht gezogen werden.

Bei der Anwendung dieser Gliederung auf beide Verbreitungszonen sakischer Gräberfelder mit Großkurganen ist festzustellen, dass kein gravierender Unterschied im Verteilungsverhältnis der Kurgane unterschiedlicher Größe (Groß- und Kleinkurgane) auf einer Nekropole vorherrscht (Tabelle I4).

Nicht auf allen untersuchten Gräberfeldern sind sämtliche Kategorien von Kurganen vertreten. Wie Tabelle I5 zeigt, treten sehr große Kurgane (über $8 \mathrm{~m}$ Höhe) in der ersten Verbreitungszone in $64 \%$ aller Nekropolen ${ }^{18} 3$ auf, dagegen kommen sie auf dem Plateau Kegen und jenseits des Kuluktau-Gebirges (zweite Verbreitungszone) nur in I9 \% der Fäller ${ }^{84}$ vor. In der ersten Verbreitungszone zählen über die Hälfte aller Großkurgane zur Kategorie der größeren Kurgane (4-8 m), nämlich bei $45 \%$ der Nekropolen $^{185}$, im zweiten lediglich bei $6 \%$ der Gräberfelder ${ }^{186}$. Auf $36 \%$ aller Gräberfelder ${ }^{187}$ bilden die großen Grabhügel (2-4 m) die zahlenstärkste Kategorie der Anlagen in den früheisenzeitlichen sakischen Nekropolen der ersten Verbreitungszone, was auf dem einzelnen Gräberfeld über die Hälfte aller Anlagen ausmacht. In Verbreitungszone zwei gilt das lediglich für $25 \%$ der Gräberfelder ${ }^{188}$. Der Fall, dass ein Teil der Goßkurgane des Gräberfeldes ausschließlich aus großen Grabhügeln (2-4 $\mathrm{m}$ ) besteht,

I8I Da das Gräberfeld am Stausee Bartogaj und die Nekropole des archäologischen Komplexes Sarytau nur aus Kleinkurganen bestehen, werden sie hier nicht berücksichtigt.

I82 Moderner Ackerbau wird im südöstlichen Siebenstromland seit der zweiten Hälfte des Iو. Jh. ausgeübt.

I83 Sieben von elf Fundorten.

I84 Drei von sechzehn Fundorten.

I85 Fünf von elf Fundorten.

I86 Einer von sechzehn Fundorten.

I87 Vier von elf Fundorten.

I88 Vier von sechzehn Fundorten. 
Abb. 36 | Gruppierung der Nekropolen nach der Anteiligkeit von Groß- und Kleinkurganen

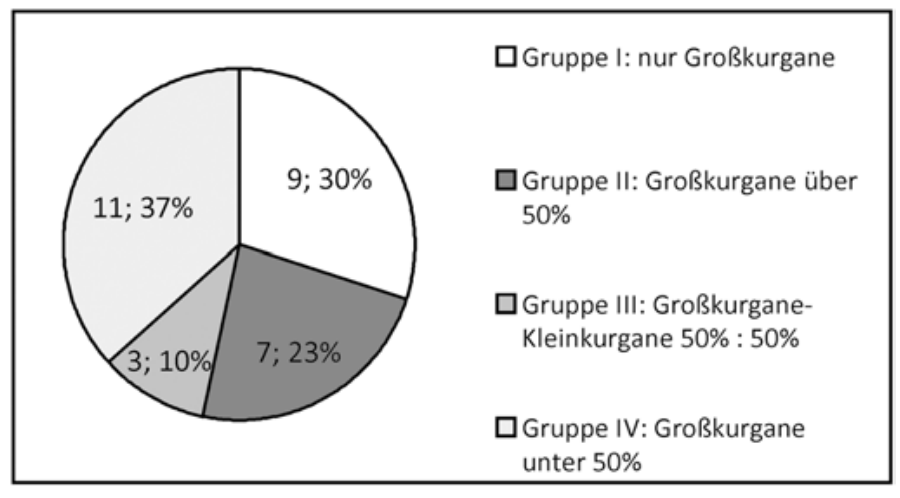

kommt nur im zweiten Verbreitungsgebiet vor (56\% der Fäller89). Eine Ausnahme stellt die Kurgangruppe Krasnyj Vostok der ersten Verbreitungszone dar (9\% der Nekropolen ${ }^{19 \circ}$ ).

\subsubsection{Abhänge der Kurgane}

Die meisten sakischen Großkurgane im südöstlichen Siebenstromland bestehen aus abgerundeten, plattformähnlichen Konstruktionen mit einer abgeflachten Kuppe. Zudem weist die Aufschüttung bei $86 \%$ dieser Kurgane ${ }^{19}$ drei steile und einen sanften Abhang auf (Abb. 27). Nur sechs Großkurgane (2\%) wurden ohne dieses architektonische Merkmal gebaut. Bei I2 \% der Großkurgane können keine Angaben bezüglich der Abhänge gemacht werden, da der Zerstörungsgrad entweder bereits zu hoch ist, oder sich keine Möglichkeit ergab, die Kurgane genauer zu untersuchen ${ }^{192}$.

Betrachtet man alle erforschten Kurgane des Untersuchungsgebietes (Abb. 37), stellt sich heraus, dass es lediglich bei $49 \%$ der Grabhügel'193 drei steile und einen sanften Abhang gibt. 353 Konstruktionen (43\%) weisen gleichmäßige Abhänge aufi 194 . Für die 64 beschädigten und zerstörten Kurgane ( $8 \%)$ können keine Aussagen bezüglich der Konstruktion und des ursprünglichen Aussehens gemacht werden.

In den meisten Fällen befindet sich der sanfte Abhang im Süden. Nach Süden wurden $40 \%$ aller untersuchten Kurgane (326 Konstruktionen) ausgerichtet. Wesentlich seltener zeigen die sanften Abhänge nach Südosten (39 Grabhügel, $5 \%$ ) bzw. Südwesten (I8 Konstruktionen, 2 \%). In wenigen Fällen kamen eine östliche, westliche und nordwestliche Ausrichtung des sanften Abhanges vor.

Die Analyse innerhalb der Verbreitungszonen zeigt, dass alle oben erwähnten Ausrichtungen des sanften Abhanges nur im Bereich der ersten Verbreitungszone anzutreffen sind. Die Kurgane der zweiten Verbreitungszone haben den sanften Abhang nur in südlicher Richtung. $98 \%$ aller Kurgane dieser Zone, bei denen die Abhänge ungleich waren, wurden mit dem sanften Abhang exakt nach Süden ausgerichtet. Der sanfte Abhang der restlichen Kurgane lag im Südosten.

Wenn man nur die Großkurgane des südöstlichen Siebenstromlandes bezüglich der Ausrichtung des sanften Abhanges untersucht, zeigt sich ganz deutlich (Abb. 38), dass es fast keine Kurgane gibt,

I89 Neun von sechzehn Fundorten.

I90 Einer von elf Fundorten.

I9I 274 Grabhügel.
I92 Elf Großkurgane des Gf. Turgen befinden sich in einem Sperrgebiet (vgl. Katalog, Gf. Turgen).

I93 396 Groß- und Kleinkurgane.

I94 Meist handelt es sich um Kleinkurgane. 
Tabelle 14 | Verhältnis der Gräberfelder mit unterschiedlichem Anteil an Groß- und Kleinkurganen innerhalb einer Nekropole mit Bezug auf die Verbreitungszonen

\begin{tabular}{|c|c|c|c|c|c|c|c|}
\hline & Gräberfeld & $\begin{array}{l}\text { Groß- } \\
\text { kurgane }\end{array}$ & $\begin{array}{l}\text { Klein- } \\
\text { kurgane }\end{array}$ & $\begin{array}{l}\text { Groß- } \\
\text { kurgane \% }\end{array}$ & $\begin{array}{l}\text { Klein- } \\
\text { kurgane \% }\end{array}$ & Zerstört & $\begin{array}{l}\text { Kurgane } \\
\text { gesamt }\end{array}$ \\
\hline \multirow{11}{*}{ 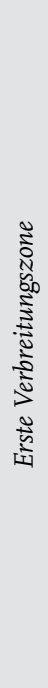 } & Asy Saga & 6 & & $100 \%$ & & & 6 \\
\hline & Krasnyj Vostok & 2 & & $100 \%$ & & & 2 \\
\hline & Ulžan & 5 & & $100 \%$ & & & 5 \\
\hline & Turgen & 33 & 2 & $94 \%$ & $6 \%$ & I & 36 \\
\hline & Issyk & $4 \mathrm{I}$ & 26 & 6г \% & $39 \%$ & I6 & 83 \\
\hline & Novoalekseevka & I2 & 8 & $60 \%$ & $40 \%$ & I & $2 \mathrm{I}$ \\
\hline & Kyzylšaryk & I7 & I6 & $52 \%$ & $48 \%$ & 4 & 37 \\
\hline & Boroldaj & 25 & 26 & $49 \%$ & $51 \%$ & I & 52 \\
\hline & Nordwestlicher Friedhof & 4 & 5 & $44 \%$ & $56 \%$ & & 9 \\
\hline & Bes Tobe & I4 & 32 & $30 \%$ & $70 \%$ & I & 47 \\
\hline & Žoan Tobe & 6 & 22 & $2 \mathrm{I} \%$ & $79 \%$ & 3 & $3 \mathrm{I}$ \\
\hline \multirow{16}{*}{ 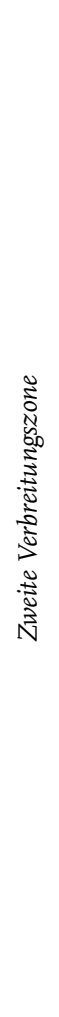 } & ${ }_{1} K{ }_{5} \mathrm{~km}$ SW von Žalauly & I & & $100 \%$ & & & I \\
\hline & ${ }_{2} K_{4} 00 m$ W von Žalauly & 2 & & $100 \%$ & & & 2 \\
\hline & Bestobe-3 & 6 & & $100 \%$ & & & 6 \\
\hline & Sarytau „A“ & 3 & & $100 \%$ & & & 3 \\
\hline & Šyrganak-2 & 8 & & $100 \%$ & & & 8 \\
\hline & Ereul & 34 & I & $97 \%$ & $3 \%$ & & 35 \\
\hline & Aufdem Plateau Kegen & 35 & 2 & $95 \%$ & $5 \%$ & & 37 \\
\hline & Togyzbulak & 6 & I & $86 \%$ & I4 \% & & 7 \\
\hline & $A k$ šoky-4 & IO & 4 & $71 \%$ & $29 \%$ & & I4 \\
\hline & Žylysaj-1 & 9 & 9 & $50 \%$ & $50 \%$ & & I8 \\
\hline & Kokpijaz & 3 & 4 & $43 \%$ & $57 \%$ & & 7 \\
\hline & Žalauly-8 & 5 & II & $3 \mathrm{I} \%$ & $69 \%$ & & 16 \\
\hline & Žalauly-2 & I7 & $5^{\mathrm{I}}$ & $25 \%$ & $75 \%$ & & 68 \\
\hline & Komplex Kiikpaj & 3 & $\mathrm{I} 2$ & $20 \%$ & $80 \%$ & & I5 \\
\hline & Aksaj-9 & 2 & 28 & $7 \%$ & $93 \%$ & & 30 \\
\hline & Aktasty-3 & 2 & $4^{\mathrm{I}}$ & $5 \%$ & $95 \%$ & & 43 \\
\hline \multirow{4}{*}{ 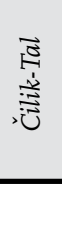 } & ${ }_{3} K{ }_{7} \mathrm{~km} W$ von Saty & 3 & & $100 \%$ & & & 3 \\
\hline & Saty & 3 & 22 & I2 $\%$ & $88 \%$ & & 25 \\
\hline & Taučilik-6 & 2 & I44 & I \% & $99 \%$ & & 146 \\
\hline & Insgesamt: & 319 & 467 & & & 27 & $8 \mathrm{I} 3$ \\
\hline
\end{tabular}


Tabelle 15 | Verhältnis der Gräberfelder mit unterschiedlichem Anteil der Kategorien der Großkurgane innerhalb einer Nekropole mit Bezug auf die Verbreitungszonen

\begin{tabular}{|c|c|c|c|c|c|c|c|c|}
\hline & Gräberfeld & $\begin{array}{l}\text { Kurgane } \\
\text { über } 8 \mathrm{~m}\end{array}$ & $\begin{array}{l}\text { Kurgane } \\
4-8 \mathrm{~m}\end{array}$ & $\begin{array}{l}\text { Kurgane } \\
2-4 \mathrm{~m}\end{array}$ & $\begin{array}{l}\text { Kurgane } \\
\text { über } 8 \mathrm{~m} \%\end{array}$ & $\begin{array}{l}\text { Kurgane } \\
4-8 \mathrm{~m} \%\end{array}$ & $\begin{array}{l}\text { Kurgane } \\
2-4 \mathrm{~m} \%\end{array}$ & $\begin{array}{l}\text { Kurgane } \\
\text { gesamt }\end{array}$ \\
\hline \multirow{11}{*}{ 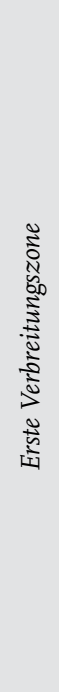 } & Nordwestlicher Friedhof & I & 3 & & $25 \%$ & $75 \%$ & & 9 \\
\hline & Asy Saga & I & 3 & 2 & $\mathrm{I} 7 \%$ & $50 \%$ & $33 \%$ & 6 \\
\hline & Žoan Tobe & I & I & 4 & I7\% $\%$ & I7\% & $67 \%$ & $3 \mathrm{I}$ \\
\hline & Boroldaj & 2 & 9 & I4 & $8 \%$ & $36 \%$ & $56 \%$ & 52 \\
\hline & Novoalekseevka & I & 8 & 3 & $8 \%$ & $67 \%$ & $25 \%$ & $2 \mathrm{I}$ \\
\hline & Issyk & 3 & I7 & $2 \mathrm{I}$ & $7 \%$ & $42 \%$ & $51 \%$ & 83 \\
\hline & Turgen & 2 & 23 & 8 & $6 \%$ & $70 \%$ & $24 \%$ & 36 \\
\hline & Ulžan & & 3 & 2 & & $60 \%$ & $40 \%$ & 5 \\
\hline & Kyzylšaryk & & IO & 7 & & $59 \%$ & $4 \mathrm{I} \%$ & 37 \\
\hline & Bes Tobe & & 2 & I2 & & I4 & $86 \%$ & 47 \\
\hline & Krasnyj Vostok & & & 2 & & & $100 \%$ & 2 \\
\hline \multirow{16}{*}{ 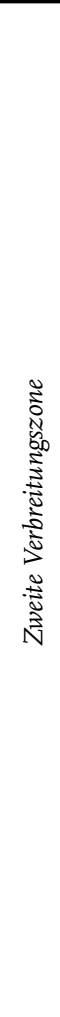 } & Auf dem Plateau Kegen & 8 & 7 & 20 & $23 \%$ & $20 \%$ & $57 \%$ & 37 \\
\hline & Togyzbulak & I & & 5 & $\mathrm{I} 7 \%$ & & $83 \%$ & 7 \\
\hline & Šrganak-2 & I & 4 & 3 & I3 \% & $50 \%$ & $37 \%$ & 8 \\
\hline & Ereul & & I8 & I6 & & $53 \%$ & $47 \%$ & 35 \\
\hline & ${ }_{2} \mathrm{~K} 400 \mathrm{~m} W$ von Žalauly & & I & I & & $50 \%$ & $50 \%$ & 2 \\
\hline & Žalauly-2 & & 3 & I4 & & I $8 \%$ & $82 \%$ & 68 \\
\hline & Akšoky-4 & & I & 9 & & I0\% & $90 \%$ & I4 \\
\hline & ${ }_{1} K{ }_{5} \mathrm{~km}$ SW von Žalauly & & & I & & & $100 \%$ & I \\
\hline & Aksaj-9 & & & 2 & & & $100 \%$ & 30 \\
\hline & Aktasty-3 & & & 2 & & & $100 \%$ & 43 \\
\hline & Bestobe-3 & & & 6 & & & $100 \%$ & 6 \\
\hline & Komplex Kiikpaj & & & 3 & & & $100 \%$ & I5 \\
\hline & Kokpijaz & & & 3 & & & $100 \%$ & 7 \\
\hline & Sarytau „A“ & & & 3 & & & $100 \%$ & 3 \\
\hline & Žalauly-8 & & & 5 & & & $100 \%$ & I6 \\
\hline & Žylysaj-1 & & & 9 & & & $100 \%$ & I8 \\
\hline \multirow{3}{*}{$\begin{array}{l}\overrightarrow{\vec{v}} \\
\frac{1}{\vec{\Xi}} \\
\dot{\vec{U}}\end{array}$} & Taučilik-6 & & 2 & & & $100 \%$ & & 146 \\
\hline & $3 K 7 k m$ W von Saty & & I & 2 & & $33 \%$ & $67 \%$ & 3 \\
\hline & Saty & & & 3 & & & $100 \%$ & 25 \\
\hline & Insgesamt: & $2 \mathrm{I}$ & II6 & I82 & & & & 813 \\
\hline
\end{tabular}



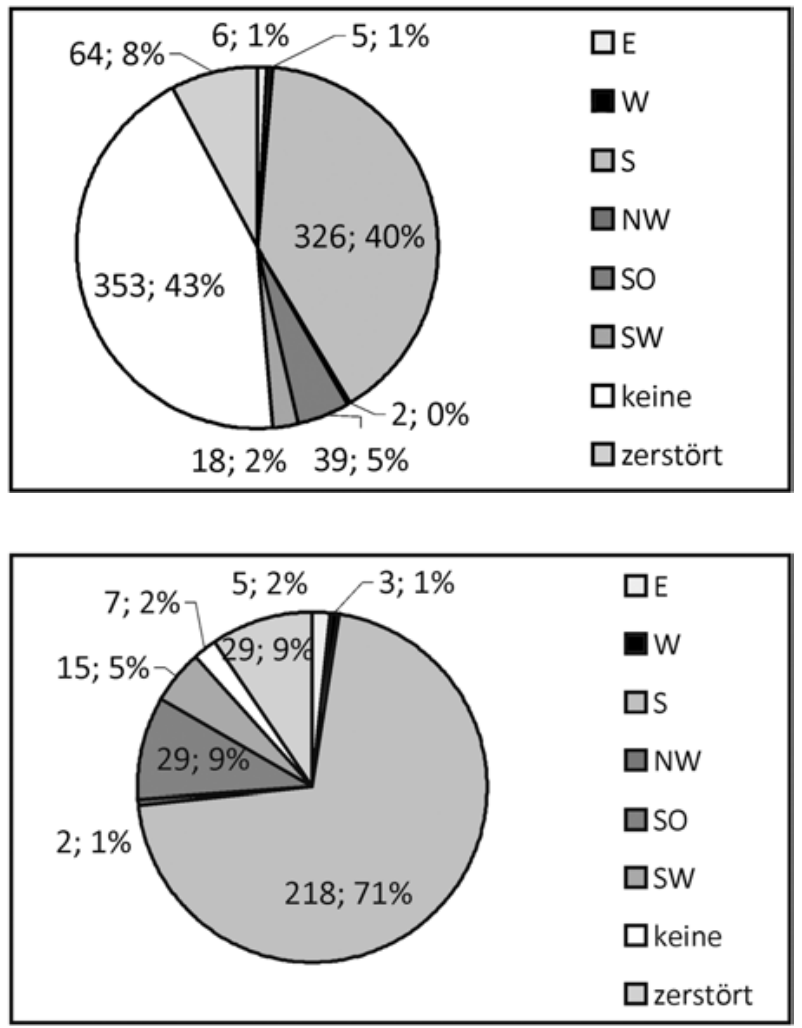

Abb. 37 | Die Ausrichtungen des sanften Abhanges aller sakischen Kurgane auf den Gräberfeldern mit Großkurganen
Abb. 38 | Die Ausrichtungen des sanften Abhanges der Großkurgane

bei denen alle Abhänge gleich geneigt sind 95 . Die exakte südliche Ausrichtung dominiert mit 71 \% (218 Kurgane). Es folgen die südöstliche (9\%, 29 Kurgane) und die südwestliche (5\%, I5 Kurgane). Ost-, West- und Nordwest-Ausrichtung kommen auch vor, allerdings nur vereinzelt.

Wie die Abbildungen 39-4I zeigen, bleibt die Tendenz zur Neigung der Abhänge bezüglich der unterschiedlichen Größenkategorien insgesamt ähnlich. Sowohl bei sehr großen Kurganen (Höhe über $8 \mathrm{~m}$ ) als auch bei größeren (4-8 m) und großen Kurganen (2-4 m) dominiert die südliche Ausrichtung des sanften Abhanges. Es spielt keine Rolle, zu welcher Verbreitungszone die Anlage gehört. Zur südlichen Ausrichtung kommen noch die südöstliche und südwestliche hinzu, so dass im Grunde genommen die südliche Ausrichtung, mit leichten Abweichungen, eine wichtige Rolle bei der Errichtung der Anlagen spielte.

Es sprechen folgende Tatsachen dafür, dass die Großkurgane bereits zum Zeitpunkt des Baus asymmetrisch, mit drei steilen und mit einem sanften Abhang angelegt wurden ${ }^{19}{ }^{6}$ :

Nicht alle Kurgane einer Kurgankette weisen eine annähernd gleiche Ausrichtung des sanften Abhanges auf, was allerdings bei einem natürlichen Verwitterungsprozess zu erwarten wäre. So stehen zum Beispiel in der Nekropole Boroldaj (vgl. Samašev u.a. 2006, 34-I28; vgl. Katalog, Gräberfeld Boroldaj) in einer Kurgankette, die von Norden nach Süden verläuft, jeweils ein Kurgan mit sanftem Abhang nach Osten (K4), nach Westen (KI) oder nach Nordwesten (K7), je zwei Grabhügel mit gleichmä-

I95 Sieben Konstruktionen, $2 \%$ aller untersuchten Großkurgane.
I96 Es wird auch die Meinung vertreten, dass die heutige äußere Form eines Kurgans ein Ergebnis der natürlichen Verwitterungsprozesse ist (Rudenko I9I8, 4; Mozolevskij/Polin 2005, 236-237). 
Abb. 39 | Die Ausrichtung des sanften Abhanges bei sehr großen Kurganen (Höhe über $8 \mathrm{~m}$ )

Abb. 40 | Die Ausrichtung des sanften Abhanges bei größeren Kurganen (4-8 m)

Abb. 41 | Die Ausrichtung des sanften Abhanges bei großen Kurganen $(2-4 \mathrm{~m})$
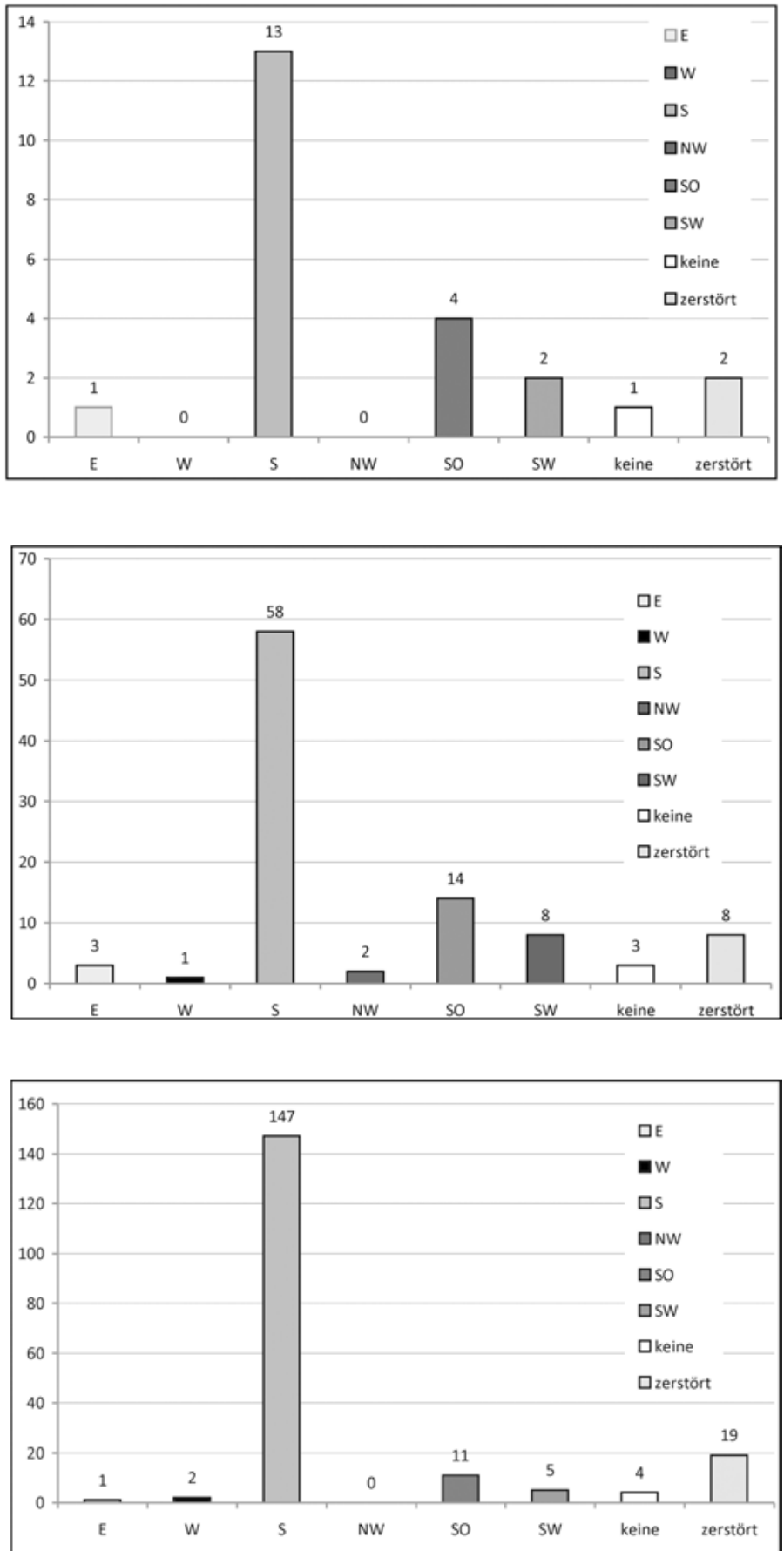

ßigen Abhängen (K5 und Kı5) oder mit sanftem Abhang nach Südosten (K9-Kıo) und fünf Tumuli mit sanftem Abhang nach Süden (K6, K8, Kı2-KI4). Bei den restlichen fünf Kurganen der Kette konnten keine Merkmale bezüglich der Abhänge festgestellt werden, da die Kurgane entweder zu klein waren (Kir, K44, K47) oder sich auf einem gesperrten Gebiet befanden (K45-K46). Eine weitere Kette desselben Gräberfeldes besteht aus jeweils einem Kurgan, dessen sanfter Abhang nach Westen (K26) bzw. Südwesten (K25) ausgerichtet wurde und zwei Kurganen, deren sanfter Abhang nach Süden (K27-K28) 
zeigte. Bei weiteren Kurganen derselben Kette wurden solche Baumerkmale nicht erwähnt (Samašev u.a. 2006, I08-IIO). Dass das Gräberfeld Boroldaj keine Ausnahme darstellt, beweist eine aus I8 Kurganen bestehende Kette auf dem Gräberfeld Issyk (vgl. Katalog, Gräberfeld Issyk). I5 Kurgane weisen hier einen sanften Abhang nach Südosten (KII, KI7-Kı9, K2I-K27, K32, K35-K36, K38), zwei Kurgane nach Süden (K39-K40) und ein Kurgan nach Nordwesten (K20) auf.

Die Theorie, dass die asiatischen Saken den europäischen Skythen sehr nahe standen und zum skythischen Kulturkreis gehörten (Parzinger 2004, 56; Mozolevskij/Polin 2005, 278, 337; Korol'kova 2006, I8-20), legt nahe, dass die Information bezüglich der „Skythen“, die aus antiken schriftlichen Quellen stammen, auch in gewisser Weise für die „Saken“ zutreffen. Einige Ereignisse, die Herodot über das „skythische“ Leben aufgeschrieben hat, treffen möglicherweise auch auf die Saken des südöstlichen Siebenstromlandes zu. Herodot berichtet über die Bestattungszeremonien skythischer Könige und über das Errichten der Königskurgane (Herodot [4-7I], 305). Über die Form solcher Kurgane sprach der griechische Historiker allerdings nicht (Herodot [4-7I], 305). Die äußere Form der Kurgane der sakischen Elite findet trotzdem einige Parallelen in den Beschreibungen Herodots, der sie allerdings nicht für Fürstenkurgane, sondern für Heiligtümer liefert (Herodot [4-62], 30I-302). Laut Herodot bauten die Skythen ihre Heiligtümer für Ares, den wichtigsten Gott des skythischen Götterpantheons, in der Form eines Hügels mit einer gevierten Ebene obenauf und mit drei steilen und einer sanften Seite. Die äußere Übereinstimmung der Kurgane der sakischen Elite, des skythischen Heiligtums auf dem Gräberfeld Bajkara in Nordkasachstan (Parzinger u.a. 2003) sowie die Ähnlichkeit mit den großen Fürstenkurganen des skythischen Kulturkreises (Mozolevskij/Polin 2005; Parzinger u.a. 20I0), zu welchem auch die Saken des Siebenstromlandes gehören, lassen vermuten, dass die großen Kurgane der sakischen Elite so gebaut wurden, dass sie zumindest in der Form den Heiligtümern des Ares ähnelten.

\subsubsection{Abdeckung der Kurgane}

Mehr als die Hälfte aller untersuchten Kurgane war auf der Oberfläche mit Steinen bedeckt (7ıo Kurgane, 74\%). Die Dichte der Steinabdeckung fällt unterschiedlich aus; es kommen einzelne Steine vor ${ }^{197}$, manchmal ist auch die gesamte Oberfläche mit Steinen bedeckt ${ }^{198}$. Die Steine haben unterschiedliche Größen ${ }^{199}$, auch Schotter wurde verwendet ${ }^{200}$. In einigen Fällen hat die Steinabdeckung einen ganz spezifischen Charakter. Zum Beispiel war die gesamte Oberfläche des Kurgans 3 vom Gräberfeld Asy Saga mit rötlichen, absichtlich zerschlagenen und gesplitterten Steinen bedeckt. Die Steinabdeckung des Kurgans 4 derselben Nekropole bestand ausschließlich aus weißen Steinen. Auf $22 \%$ aller Kurgane (2I4 Grabhügeln) finden sich an der Oberfläche gar keine Steine²or. Bei vier Großkurganen ${ }^{202}$ (г \%) wurden im unteren Bereich der Kurganaufschüttung mittelgroße Steine festgestellt, die Kurgankuppe jedoch wies gar keine Steine auf. Bei den restlichen 27 Bauten (3\%) konnten aufgrund eines hohen Zerstörungsgrades keine Aussagen gemacht werden.

197 z.B.: Kurgan 2 des Gf. Asy Saga (vgl. Katalog, Gf. Asy Saga, Kurgancharakteristik).

I98 z. B.: Kurgan 3 des Gf. Asy Saga (vgl. Katalog, Gf. Asy Saga, Kurgancharakteristik).

I99 z. B.: $60 \times 40 \times 30 \mathrm{~cm}$ auf dem Kurgan Io des Gf. Kyzylšaryk (vgl. Katalog, Gf. Kyzylšaryk, Kurgancharakteristik).
200 z.B.: Kurgan 4 des Gf. Saty (vgl. Katalog, Gf. Saty, Kurgancharakteristik).

$20 \mathrm{I}$ z. B.: Kurgane 7-9 des Gf. Akšoky-4 (vgl. Katalog, Gf. Akšoky-4, Kurgancharakteristik).

202 Gf. Asy Saga, K6; Gf. Issyk, K40; Gf. Kyzylšaryk, K8 und K9. 
Die Untersuchung der Großkurgane des Arbeitsgebietes zeigt, dass Kurgane mit Steinen an der Oberfläche dominieren (I7I Grabhügel, 54\% aller Großkurgane). Das ist knapp über die Hälfte der Anlagen. Die Großkurgane, die gar keine Steine an der Oberfläche aufweisen, nehmen auch einen sehr großes Anteil ein (I44 Objekte, 45\%). Die übrigen vier Großkurgane mit einer teilweisen Abdeckung wurden bereits oben erwähnt.

Solche Unterschiede in der Konstruktion der Kurgane, besonders der Großkurgane, können mit der letzten aktiven Bauphase erklärt werden. Über das endgültige Aussehen eines Kurgans wurde entschieden, indem die Oberfläche am Ende durch eine Steinabdeckung oder eine Lehmschicht versiegelt wurde.

\subsubsection{Aufbau der Kurgane: Funde und Befunde}

Erkenntnisse zu Konstruktion und Aufbau der Kurgane, der Stratigraphie der Aufschüttung und Informationen zur Grabgrube bzw. den Grabgruben können aus den Ergebnissen der alten Ausgrabungen gewonnen werden. Auf den erforschten Gräberfeldern wurden 69 Kurgane festgestellt, die zum Teil oder komplett ausgegraben worden sind. Es handelt sich um 2I Groß-203 und 48 Kleinkurgane ${ }^{204}$. Zudem wurde teilweise die Peripherie dreier Großkurgane ${ }^{205}$ untersucht. Trotz der relativ großen Zahl der untersuchten Konstruktionen bleibt der heutige Kenntnisstand sehr spärlich, da die meisten in der Nachkriegszeit ausgegrabenen Kurgane nicht immer oder nur unvollständig dokumentiert wurden.

Es wurde festgestellt, dass die Kurganaufschüttung der Großkurgane aus drei bis vier abwechselnden Schichten lehmigen Sandes bzw. Kieselsandes und Steinpackungen, die überwiegend aus mittelgroßen Kieselsteinen bestanden, aufgebaut war. Die oberste Schicht bildete gelegentlich eine Steinpackung. In einigen Fällen bedeckten die Steine nur die untere Hälfte der Kurganabhänge, so dass die Kuppe kahl blieb. Gelegentlich fand man auf dem gewachsenen Boden ein Steinpflaster, und in den oberen Schichten wurde eine Doppelsteinreihe angelegt, wobei die Steine durch einen Lehmmörtel fi-

203 Zehn Großkurgane wurden auf dem Gf. Issyk ausgegraben (vgl. Katalog, Gf. Issyk, Kurgancharakteristik und Forschungsgeschichte) - Kurgane 40 und $4 \mathrm{I}$ (siehe Katalog), Kurgan „Issyk“ und vier weitere Großkurgane (vgl. Akišev K. A. 1978), „Kurgan beim Dorf Issyk“ (vgl. Maksimova I97I), „Kurgan auf dem Gf. Issyk, I. Stelle“ (vgl. Nurmuchanbetov u.a. 1992, 29, 32, Abb. 4) und Kurgan № 6 (Bajpakov u. a. 2005c, 7-8, 26-27, 29, Foto I8-20, Abb. 7-8). Sechs Großkurgane wurden in der Nekropole Turgen untersucht (vgl. Katalog, Gf. Turgen, Kurgancharakteristik und Forschungsgeschichte) - Kurgan 3 (siehe Katalog; vgl. Dublickij I939a, 5-8; vgl. Dublickij I946a, I3, I6-I9, Zeichenblätter I-5; vgl. Nurmuchanbetov 1998, 35-37, Abb. 6-7; vgl. Gass 2011a, 64-65, Fig. 9-13), Kurgan 7 (siehe Katalog, vgl. Nurmuchanbetov I998, 27-50), Kurgane I5 und I6 (siehe Katalog, vgl. Nurmuchanbetov I992, 30), „Kurgan am Steilhang des Flusses Turgen“ (vgl. Bernštam/Dublickij I936, 5-6). Fünf Großkurgane wurden auf dem Gf. Ulžan ausgegraben (vgl. Katalog, Gf. Ulžan, Kurgancharakteristik) - Kurgane I-5 (siehe Katalog, vgl. Nurpeisov u.a. 2008, 225-240).

20423 Kleinkurgane wurden auf der Nekropole Issyk untersucht (vgl. Katalog, Gf. Issyk, Forschungsgeschichte) Kleinkurgane I-4, 7-9, I2-I3, I5-20, 25, 27, 29-3I, 35 (vgl. Čarikov I960), Kurgane № 4 und № 5 (Bajpakov u.a. 2005c, 6-7, Foto II-I6). Vier Kleinkurgane wurden auf dem Gf. Novoalekseevka ausgegraben (vgl. Katalog, Gf. Novoalekseevka, Forschungsgeschichte) - Kurgane № I, № 2, № 4, № 7 (vgl. Kopylov i956, 2-I8, Taf. I, 4-6). 2I Kleinkurgane wurden in der Nekropole Žoan Tobe untersucht (vgl. Katalog, Gf. Žoan Tobe, Kurgancharakteristik und Forschungsgeschichte) - Kurgane 8 und 9 (siehe Katalog), Kleinkurgane 3I (5), 34 (6) und 36 (9) (vgl. Carikov I956a, 29-36), Kleinkurgane 8 und I2 (vgl. Maksimova I956a, 3-8), Kleinkurgane I (43), 2 (44), 3 (62), 4 (48), 5 (77), 6 (78), 6I, 8I, 84 (5), 94 (7) (vgl. Carikov I956b, 27-55), Kleinkurgane I, 4, 6 und I8 (vgl. Pacevič I956, 32-38) und die Kleinkurgane Io, 49, 70 (vgl. Maksimova I960a, 62).

205 Gf. auf dem Plateau Kegen, Kurgan 2 (vgl. Katalog, Gf. auf dem Plateau Kegen, Kurgancharakteristik; vgl. Nagler u.a. 2010, 49-54, Abb. 37; vgl. Gass 2011b, 216, Abb. 20-2I), Gf. Turgen, Kurgan 3 (siehe Katalog; vgl. Dublickij I939a, 5-8; vgl. Dublickij I946a, I3, I6-I9, Zeichenblatt 2) und Gf. Žoan Tobe, Kurgan I (vgl. Katalog, Gf. Žoan Tobe, Kurgancharakteristik; vgl. Nagler 2009; vgl. Samašev u.a. 2009; vgl. Nagler u.a. 20I0, 49-54, Abb. 38-39; vgl. Gass 20iıa, 67-68, Fig. 23; vgl. Gass 20Irb, 2I3, Abb. 9). 

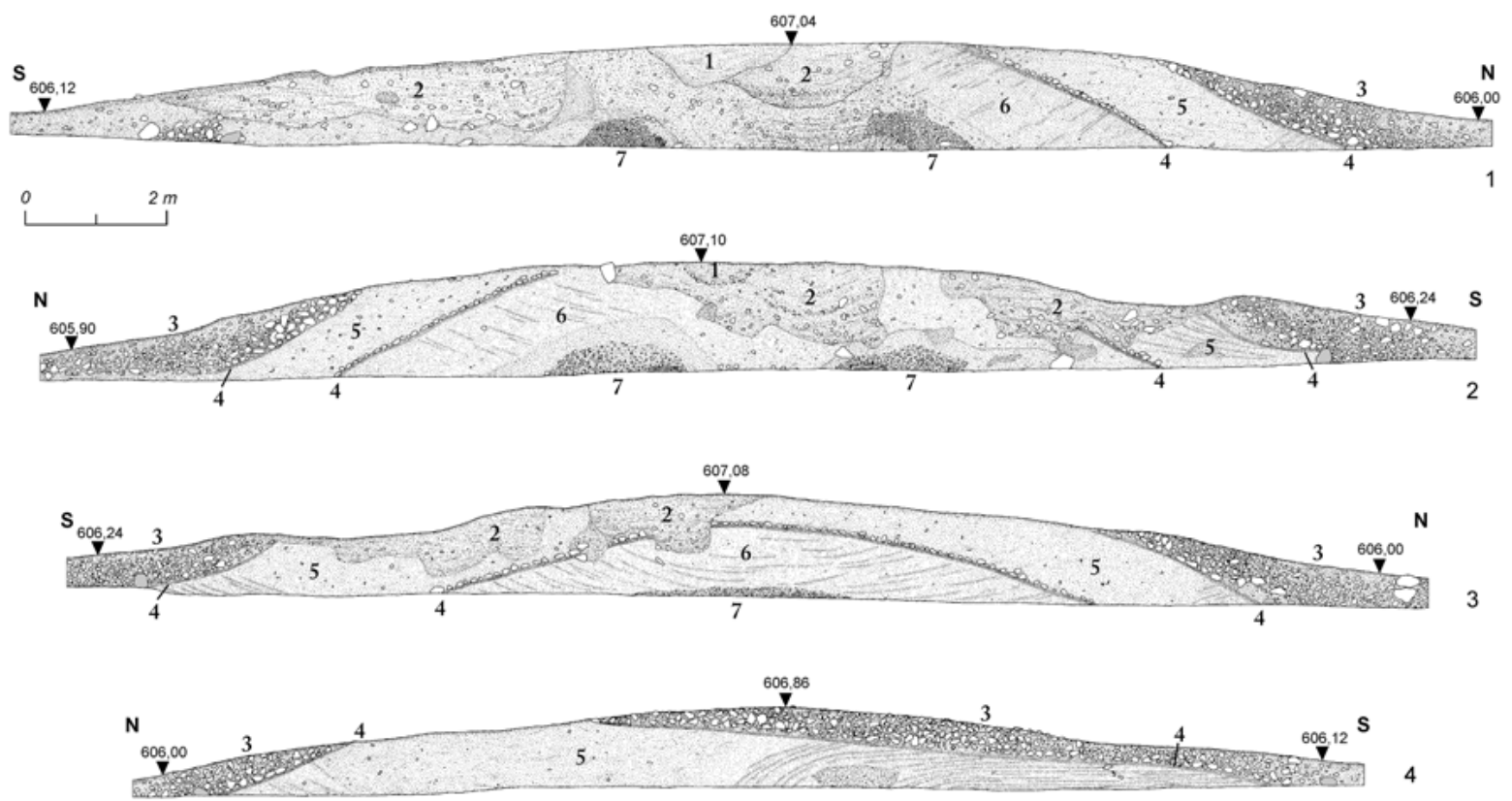

Abb. 42 | Gf. Žoan Tobe, Kurgan 8. Profile (Zeichnung M.-R. Boroffka). Legende: I.-2. Schichten des Raubtrichters; 3. Kurganpanzer bzw. Steinpackung; 4. Nasser Lehm? bzw. harter, feiner, dünnflüssiger Lehm; 5. Harter, z.T. gestampfter Lehm mit Sand- und Kiespartikeln; 6. Sandiger, feiner Lehm; 7. Kies von der Grabaushub

xiert wurden (Gf. Turgen, K3 ${ }^{206}$ ). Sowohl bei einigen Großkurganen (Gf. Turgen, K7; Gf. Ulžan, K2207), als auch bei Kleinkurganen (Gf. Žoan Tobe, K8 und $\mathrm{K}^{208}$ ), deren Höhe über I $\mathrm{m}$ maß, wurde im Zentrum des Kurgans, über der Grabgrube eine lehmige Halbkugel errichtet (Abb. 42). Diese Halbkugel wurde dann mit dünnflüssigem Lehm verschmiert (Abb. 43). Darüber folgte eine Steinpackung. In den meisten Fällen wurde um und auf diese mit Steinen verstärkte Halbkugel entweder gestampfter Lehm oder lehmiger Sand aufgebracht, so dass die ganze Konstruktion eine plattformähnliche bzw. pyramidenähnliche Form mit abgeflachter Kuppe erhielt. Bereits in dieser Bauphase wurde ein Abhang wesentlich sanfter als die restlichen gebaut. Dann folgten, wie beschrieben, dünnflüssiger Lehm oder Lehmmörtel-, Lehm- und Steinschichten. Letztendlich hatte der Kurgan meist eine abgerundete, plattformähnliche Form mit abgeflachter Kuppe sowie drei steile und einen sanften Abhang. Die Konstruktion wurde mit besagter Steinschicht abgedeckt.

Die Details des Kurganaufbaus kann man anhand der 2008 untersuchten Kurgane 8 und 9 vom Gräberfeld Žoan Tobe genauer betrachten ${ }^{209}$. Beide Kurgane befanden sich am Westrand der Nekropole

206 Siehe Katalog, Gf. Turgen, Kurgancharakteristik.

207 Siehe Katalog, Gf. Turgen und Gf. Ulžan, Kurgancharakteristik.

208 Siehe Katalog, Gf. Žoan Tobe, Kurgancharakteristik.

209 Kleinkurgane 8 und 9 des Gf. Žoan Tobe sowie die Peripherie des Großkurgans I wurden im Sommer 2008 durch die Kasachisch-Deutsche Ausgrabung untersucht. Die Ausgrabung fand im Rahmen der internationalen Kooperationsarbeit des Archäologischen Institutes „A. Ch. Margulan“ (Almaty/ Kasachstan), der Stiftung Preußischer Kulturbesitz (Berlin), des Deutschen Archäologi- schen Institutes (Berlin) und des Exzellenzclusters Topoi (Berlin) statt. Die Leitung auf kasachischer Seite hatte Z. Samašev inne, auf deutscher Seite lag die Leitung bei H. Parzinger, A. Nagler und M. Nawroth. Weitere Teilnehmer der Expedition waren M.-R. Boroffka, A. Čotbaev, B. Nurmuchanbetov und A. Gass. Die zeichnerische Dokumentation oblag M.-R. Boroffka. Die fotografische Dokumentation übernahmen Z. Samašev, H. Parzinger, M.-R. Boroffka und M. Nawroth. Die Erdarbeiten leisteten die Arbeiter aus dem Dorf Masak. Allen Beteiligten sei an dieser Stelle für ihren Einsatz herzlich gedankt. 
Abb. 43 | Gf. Žoan Tobe, Kurgan 8. Profil I. Fragment. Verschmierter dünnflüssiger Lehm und darauf folgende Steinpackung

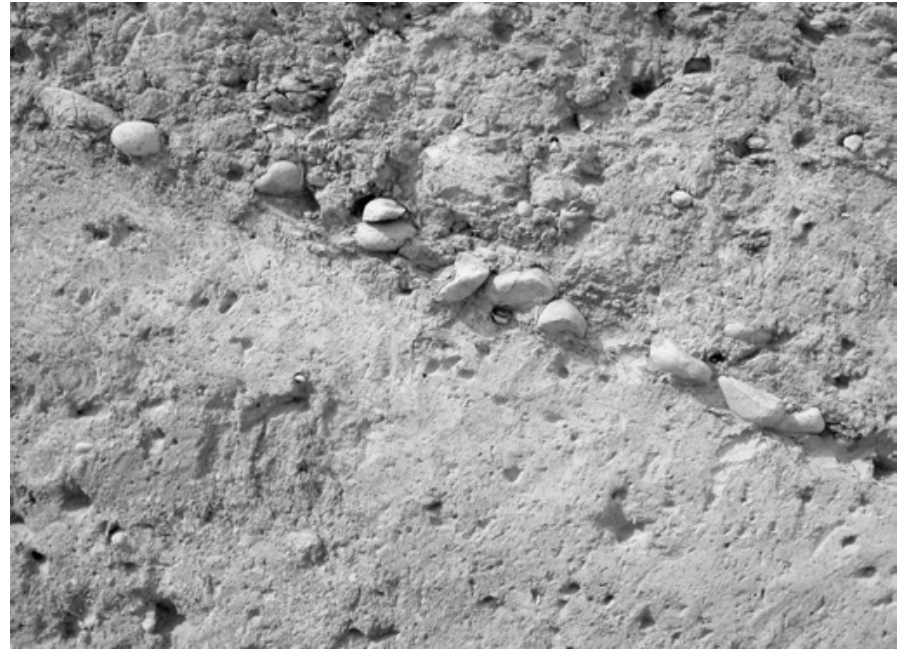

und bildeten eine nordsüdlich verlaufende Kette ${ }^{210}$. Die Oberfläche beider Kurgane war durch die landwirtschaftlichen Aktivitäten gestört. Kurgan 8, der in der Kette nördlich stand, wies eine abgerundete mit abgeflachter Kuppe Form, die durch langjähriges Pflügen entstanden war. Im südwestlichen Bereich des Kurgans fand sich ein Raubtrichter. Mit einer Höhe von I,2 m und einem Durchmesser von ca. 2I m kann dieses Bodendenkmal als ein Kleinkurgan betrachtet werden. Die geophysikalische Aufnahme ${ }^{2 I I}$ zeigte dagegen eine viereckige Struktur innerhalb des Kurgans (Abb. 44).

Kurgan 9, der 30 m südlich vom Kurgan 8 stand, wies einen Durchmesser von iو m und eine Höhe von I,2 $\mathrm{m}$ auf. Der Grabhügel hatte eine abgerundete Form mit einer abgeflachten Kuppe, die, wie beim Kurgan 8, durch langjähriges Pflügen verformt worden war. In der Mitte des Kurgans fand sich ein Raubtrichter.

Beide Kurgane wurden fast gleichzeitig mittels eines Radladers ausgegraben, dessen Ladeschaufel ein Fassungsvermögen von $2 \mathrm{~m} 3$ hat. Dadurch konnten beträchtliche Mengen Erdreich abtransportiert werden. Der Einsatz der technischen Hilfsmittel erlaubte es schnell und sparsam und ohne großen Aufwand der Arbeitskräfte, solche Bauten zu untersuchen. Dabei wurden nordsüdlich verlaufende parallele Schnitte, die durch Profilstege voneinander getrennt waren, durch die Kurgane 8 und 9 angelegt. Die Breite der Schnitte maß 2,6 m und der Profilstege I,8-2,5 m. Solche Verfahren wurden bereits in den Jahren I997-99 und 2004-06 bei der Freilegung des großen Kurgans von Bajkara in Nordkasachstan (Parzinger u.a. 2003, 4-I2) und des Großkurgans von Barsučij Log in Chakassien, Russland (Parzinger u.a. 20I0, I77-I78) erfolgreich angewandt. Am Kurgan 8 (Abb. 45) wurden drei Schnitte angelegt, so dass zwei Profilstege mit demensprechend vier Profilen entstanden sind. Ein Profilsteg mit den Profilen I und 2 verlief mittig durch den Kurgan, der zweite mit den Profilen 3 und 4 in der Westhälfte des Grabhügels.

Der Kurgan 9 (Abb. 46) bekam vier Schnitte, so dass dort drei Profilstege mit insgesamt sechs Profilen entstanden sind. Der mittlere Steg mit den Profilen 3 und 4 verlief durch das Zentrum des Kurgans. Ein Profilsteg mit den Profilen I und 2 wurde in der Osthälfte der Konstruktion gelassen und der

210 Siehe dazu Abb. 202 im Katalog.

2II Die geomagnetische Prospektion wurde von PD Dr. Jörg Faßbinder und Dr. Tomasz Gorka, Bayerisches Landes- amt für Denkmalpflege (München) durchgeführt, denen ich für die Überlassung der Daten zu Dank verpflichtet bin. 


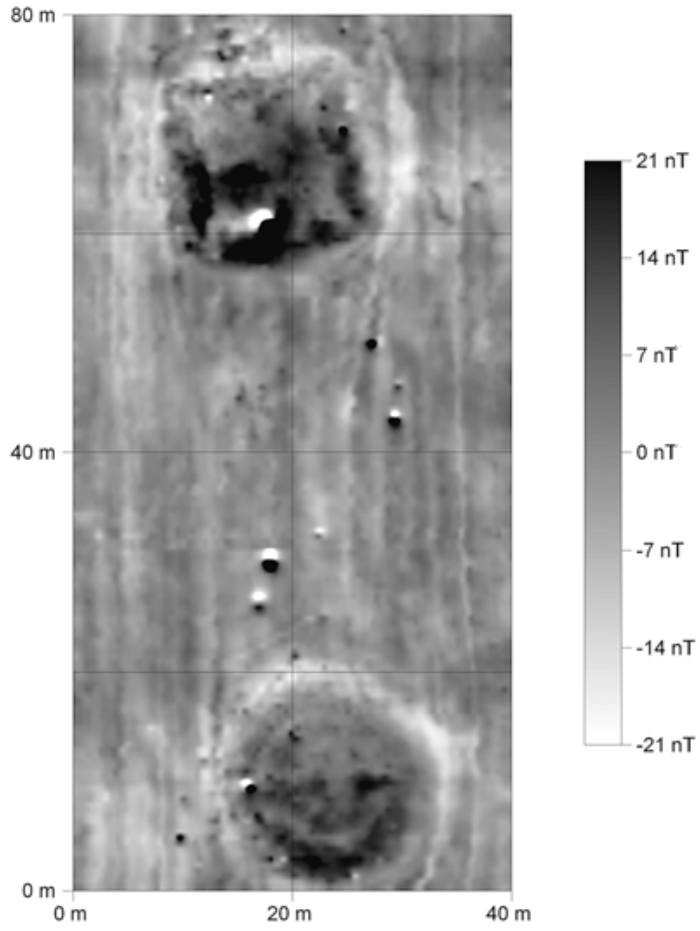

Abb. 44 | Gf. Žoan Tobe. Kurgane 8 (oben) und 9 (unten). Ergebniss der Magnetomessung (Caesium Smartmag $\mathrm{SM}_{4} \mathrm{G}$ special Magnetometer in duo-sensor Konfiguration, Empfindlichkeit +/- Io Picotesla). Dynamik +/- 2I nT in 256 Graustufen von Schwarz bis Weiß; reduziert auf den Mittelwert, Messgitter $20 \times 20$ Meter; Messpunktdichte I2,5 $\times$ I2, $5 \mathrm{~cm}$; Das Erdmagnetische Totalfeld im August 2008 ca. $55520+/-20$ Nanotesla. Norden ist oben (Bildnachweis und Datenverarbeitung: J. Faßbinder BLfD München)

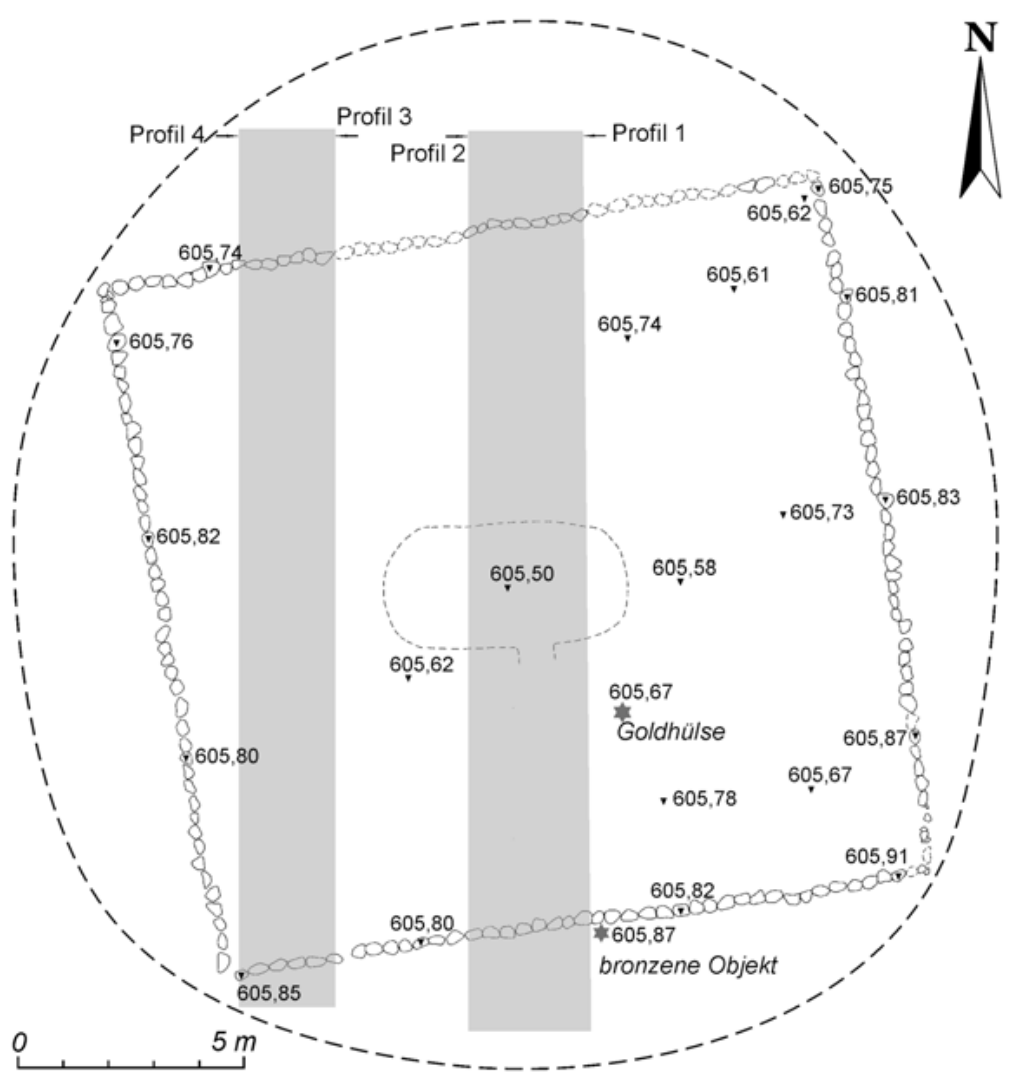

Abb. $45 \mid$ Gf. Žoan Tobe. Kurgan 8. Plan des Kurgans mit genauer Lage der Profilstege und Profile (Zeichnung M.-R. Boroffka) 
Abb. 46| Gf. Žoan Tobe. Kurgan 9. Plan des Kurgans mit genauer Lage der Profilstege und Profile (Zeichnung M.-R. Boroffka)

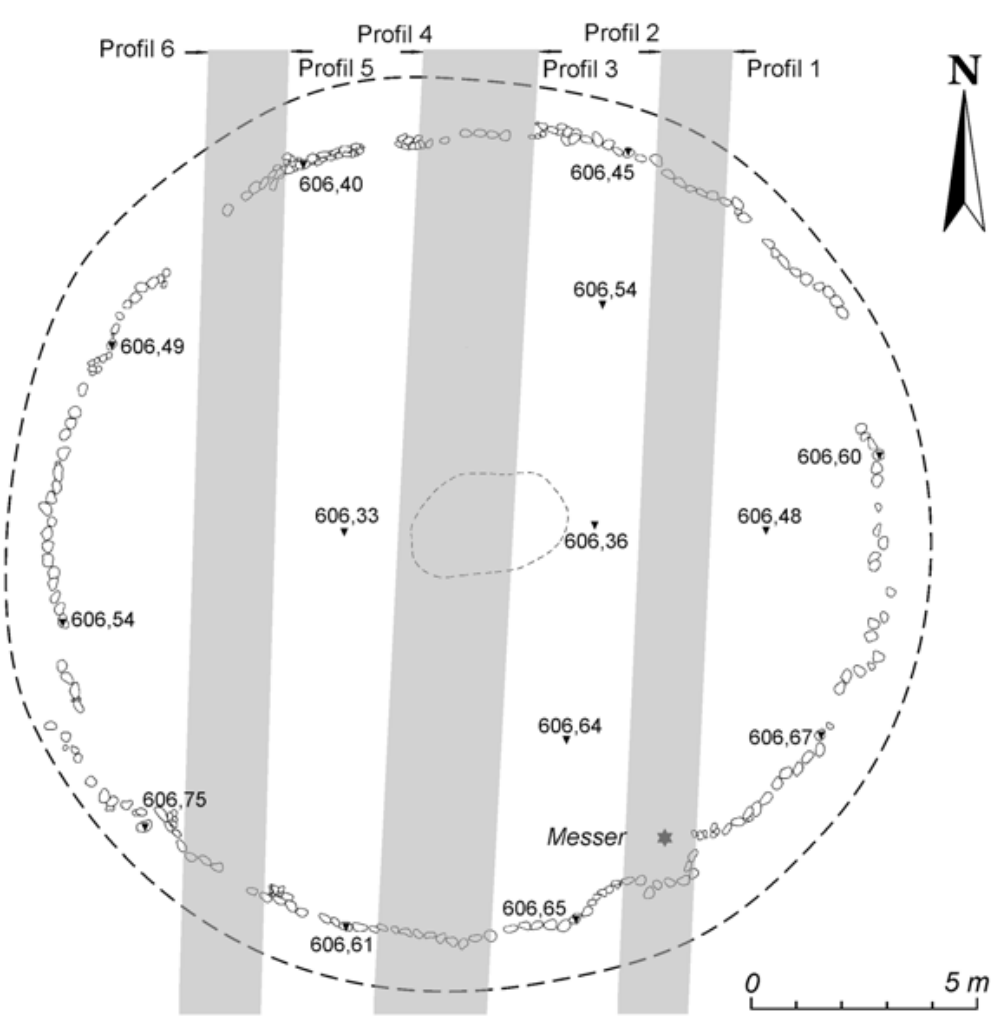

letzte mit den Profilen 5 und 6 fand sich in der Westhälfte des Grabhügels. Die Profilstege wurden nordsüdlich orientiert, erhielten jedoch durch die Beschaffenheit der Oberfläche des Kurgans, der mit mittelgroßen Steinen bedeckt worden war, eine leichte Ost-West-Abweichung.

Auf beiden Kurganen wurde die Aufschüttung in den Schnitten in etwa 15-20 cm starken Schichten abgetragen. Nach jeder Fahrt des Radladers wurden die entstanden Plana zwischen den Stegen auf Befunde und Verfärbungen untersucht. Kam eines von beiden vor, wurden weitere Untersuchungen per Hand weitergeführt und im Nachhinein dokumentiert. Sowohl westliche als auch östliche Kurgansegmente wurden soweit freigelegt, bis der Kurganrand sichtbar wurde. Daher sind die Segmentteile beider Kurgane breiter als die Schnitte des mittleren Bereichs. Durch diese Vorgehensweise gelang es, beide Kurgane zügig, und gleichzeitig sorgfältig zu erforschen.

Die angewandte Methode ermöglicht es - im Gegensatz zur Untersuchung in vier großen Sektoren mit zwei kreuzförmig angelegten Profilstegen - mehr Profile zu schaffen. Mehrere parallel verlaufende Stege bieten einen besseren Blick auf die Kurganaufschüttung und erlauben ein besseres Verständnis für den Aufbau des Kurgans zu gewinnen. Die Aufschüttung, die in Scheiben geschnitten ist, stellt eine Art „überdimensionale Computertomographie“ dar (Parzinger u.a. 20I0, I77). Der Einsatz eines Radladers eignete sich für die Durchführung einer Ausgrabung wesentlich besser als der einer Planierraupe, da diese den Aushub bewegt und vor sich herschiebt. Dadurch wird die Untersuchungsfläche zugeschüttet. Der Radlader schneidet die Aufschüttung des Kurgans und transportiert sie in der Ladeschaufel gleich weg. Durch dieses Verfahren bleibt die Untersuchungsfläche frei vom Aushub und man kann Verfärbungen gleich erkennen.

Die sorgfältig dokumentierten Profile beider Kurgane ergaben ein ähnliches Bild in Hinblick auf den Aufbau der Kurganaufschüttung (Abb. 42 und Abb. 47). Die meisten Informationen zum Aufbau 

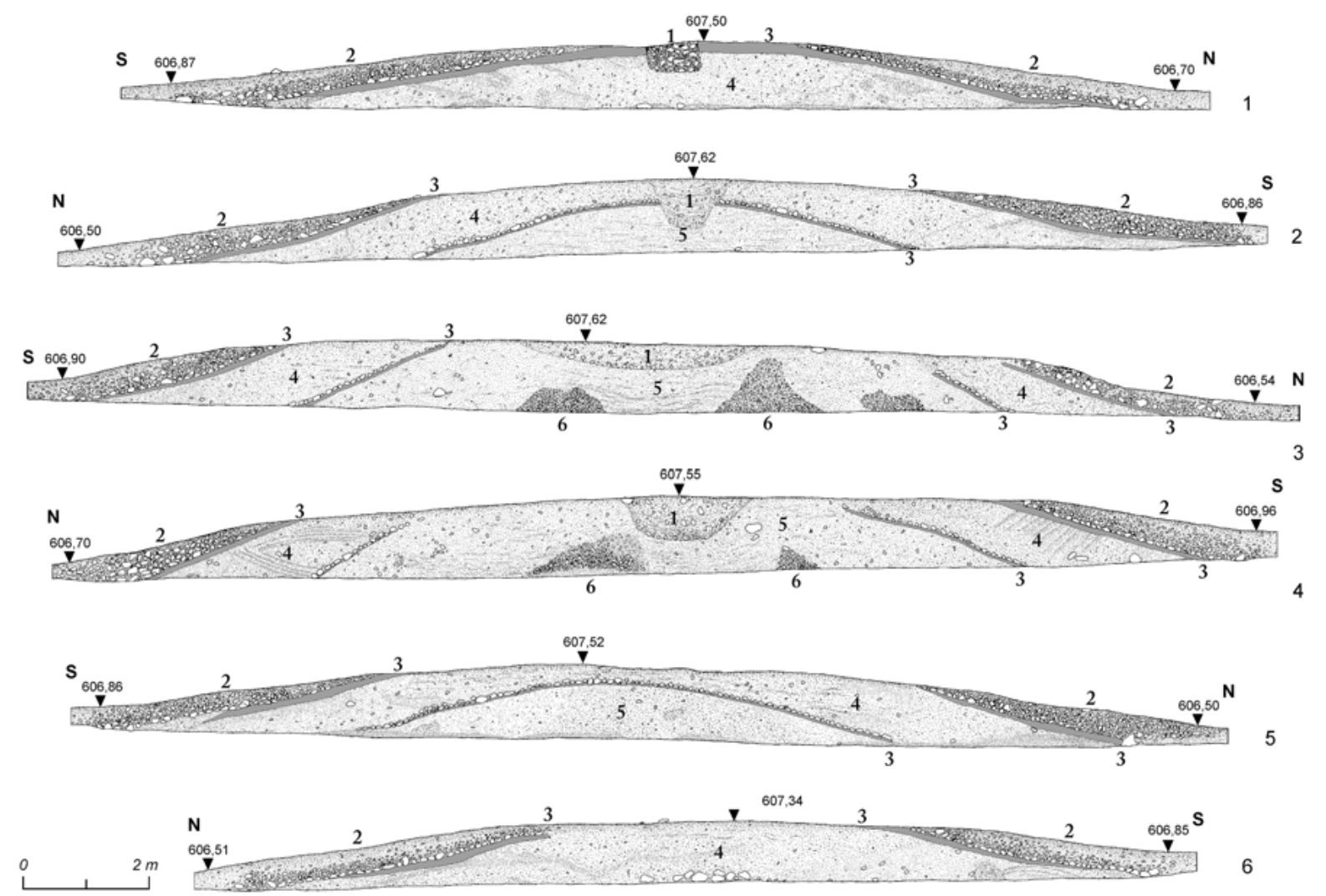

Abb. 47 | Gf. Žoan Tobe, Kurgan 9. Profile (Zeichnung M.-R. Boroffka). Legende: I. Verfüllung des Raubtrichters; 2. Kurganpanzer bzw. Steinpackung; 3. Nasser Lehm? bzw. harter, feiner, dünnflüssiger Lehm; 4. Harter, z.T. gestampfter Lehm mit Sand- und Kiespartikeln; 5. Sandiger, feiner Lehm; 6. Kies von der Grabaushub

der Kurgane liefern die Profile 2 und 3 vom Kurgan 8 (Abb. 42) und Profile 3 und 4 vom Kurgan 9 (Abb. 47), die durch das Zentrum der jeweiligen Kurgane verliefen.

Im Zentrum beider Kurgane befand sich eine Grabgrube. Der Aushub der Grabgrube lag in beiden Fällen, sowohl bei Kurgan 8 als auch bei Kurgan 9, hauptsächlich nördlich und südlich von ihr, wobei der Aushub bei Kurgan 9 mächtiger als beim Kurgan 8 ist. Über den Aushub der Grabgrube baute man eine Halbkugel, die aus feinem sandigem Lehm bestand und die teils in feinen Streifen sorgfältig aufgetragen wurde. Beim Kurgan 8 wies sie einen Durchmesser von II,7 m und eine Höhe von ca. I,7 m auf; beim Kurgan 9 bis ı, 6 m im Durchmesser und I,2 $\mathrm{m}$ in der Höhe. Diese Halbkugel wurde mit feinem, hartem, dünnflüssigem Lehm versiegelt, dessen Dicke 8-Io $\mathrm{mm}$ im oberen Bereich und bis zu $2 \mathrm{~cm}$ unten beim Halbkugelfuß betrug (Abb. 43). Eventuell wurden gleichzeitig auf dem dünnflüssigen Lehm mittelgroße und kleine Steine angesetzt. Als nächster Schritt folgte eine ca. $০, 4 \mathrm{~m}$ bis I, $\mathrm{m}$ mächtige Lehmschicht, teils fein und dicht, teils sandiger und grober, so dass es sich dann eher um mit Kies vermischten, lehmigen Sand handelte. Am Kurganrand wurde diese Schicht besonders verstärkt.

Bereits in dieser Bauphase sind erste Unterschiede bei der Kurgangestaltung festzustellen. Beim Kurgan 8 baute man mittels dieser Lehmschicht eine viereckige, pyramidenähnliche Konstruktion mit abgeflachter Kuppe, so dass der südliche Abhang wesentlich sanfter als die anderen Hänge war. Kurgan 9 bekam durch eine ähnliche Aufbauschicht eine abgerundete Form mit abgeflachter Kuppe. Ob 
die Abhänge des Kurgans 9 alle gleich oder asymmetrisch waren, lässt sich aufgrund des schlechten Erhaltungszustands der Kurganaufschüttung nicht mehr rekonstruieren. Dem Profil I zufolge ist der Südabhang sanft abfallend (Abb. 47,I), jedoch weist das Profil 6 beim Nordabhang ein identisches Gefälle auf (Abb. 47,6). In derselben Phase wurden um den Kurgan 8 auf dem gewachsenen Boden als äußere Grenze des Kurgans mittelgroße und große Steine in einer Reihe aufgelegt, jeweils eine in jeder Haupthimmelsrichtung, so dass eine Art viereckige Umfriedung entstand (Abb. 45, Abb. 48-49). Die Seitenlänge der Umfriedung beträgt I5,7 m. Sie wurde grob an den vier Haupthimmelsrichtungen, leicht nach Westen aus der Nord-Süd-Achse verschoben, orientiert.

Die Abhänge sowohl der gebauten kegelstumpfartigen Pyramide des Kurgans 8 als auch der abgerundeten halbkugeligen Konstruktion des Kurgans 9 wurden im nächsten Bauabschnitt mit feinem dünnflüssigem Lehm versiegelt, dessen Dicke bis zu I cm beim Kurgan 8 oder bis zu I, $8 \mathrm{~cm}$ beim Kurgan 9 reichte. Darauf folgte eine Steinschicht aus mittelgroßen Steinen und Kieselsteinen, die mit lehmigem Sand vermischt und durch dünnflüssigen Lehm an den Kurgan „geklebt“ wurde (Abb. 50). Diese Steinschicht stellte die letzte Bauphase des Grabhügels dar und gestaltete den Kurgan 8 endgültig zu einer viereckigen, pyramidenähnlichen Konstruktion mit abgeflachter Kuppe und den Kurgan 9 zu einem abgerundeten Grabhügel mit abgeflachter Kuppe.

Das Profil I des Kurgans 8 (Abb. 42,I) weist massive Störungen in seinem mittleren Bereich und in seiner Südhälfte auf. Wie bei den zuvor beschriebenen Profilen zeigt auch Profil I den kiesartigen Aushub der Grabgrube südlich und nördlich von ihr. Über dem Aushub liegt eine I,2-I,3 m mächtige Schicht des sandigen feinen Lehms von der Halbkugel, die vor allem in der Nordhälfte gut erhalten ist. Hier folgt eine ähnliche, stratigraphische Situation wie in den Profilen 2 und 3. Die sandige Lehmkugel wurde mit feinem dünnflüssigem Lehm versiegelt; darauf folgte eine Steinschicht. Diese wurde durch eine bis zu I,o m mächtige Schicht aus hartem, zum Teil gestampftem Lehm bedeckt, der auch Sandund Kiesanteile aufwies. Darauf folgte eine bis zu I cm dicke dünnflüssige Lehmschicht, auf die die mittelgroßen Steine gesetzt worden sind. Die mittelgroßen Steine und Kieselsteine, die mit lehmigem Sand gemischt wurden, stellen den Steinpanzer des Kurgans dar. Der mittlere Bereich des Profils ist fast komplett durch einen Raubschacht beschädigt. Zudem sieht man an den Störungsschichten mehrere Angriffe auf den Kurgan. Da die tiefergreifende, nördlich von der Mitte angelegte Störung stark sedimentiert worden war, könnte es sich um eine antike Beraubung handelt, die möglicherweise bald nach der Bestattung geschah. Bei der südlich von der Mitte angelegten Störung könnte es sich um einen wiederholten neuzeitlichen Versuch der Grabberaubung handeln. Dies belegt ein in der Störung belassenes, beiges, scheibengedrehtes, dickwandiges Gefäß der Neuzeit (I7.-I9. Jh.), das zum größten Teil erhalten war'212. Die Südhälfte des Profils weist nur die Störungsschichten auf. Lediglich am Südrand des Profils kommt eine Steinschicht der Kurganabdeckung vor.

Im Profil 4 des Kurgan 8 (Abb. 42,4) wird die Konstruktion vom Grabhügel an seinem Westrand sichtbar (Abb. 45). Hier ist eine I5,5 $\mathrm{m}$ lange und max. I,I $\mathrm{m}$ mächtige Schicht des harten zum Teil gestampften Lehms zu sehen, die in der Nordhälfte mit Kies gemischt worden und in der Südhälfte sehr fein und dicht war. Diese Schicht gehörte zur zweiten, äußeren Lehmüberlagerung des Kurgans, die den inneren lehmigen Kern abdeckte. Auf der darauffolgenden dünnflüssigen Lehmschicht der äußeren Versiegelung, die Maße von $8 \mathrm{~mm}$ bis zu I8 mm aufwies, wurden mittelgroße Steine aufgesetzt, die noch zusätzlich durch kleinere Steine und lehmigen Sand verstärkt wurden. 


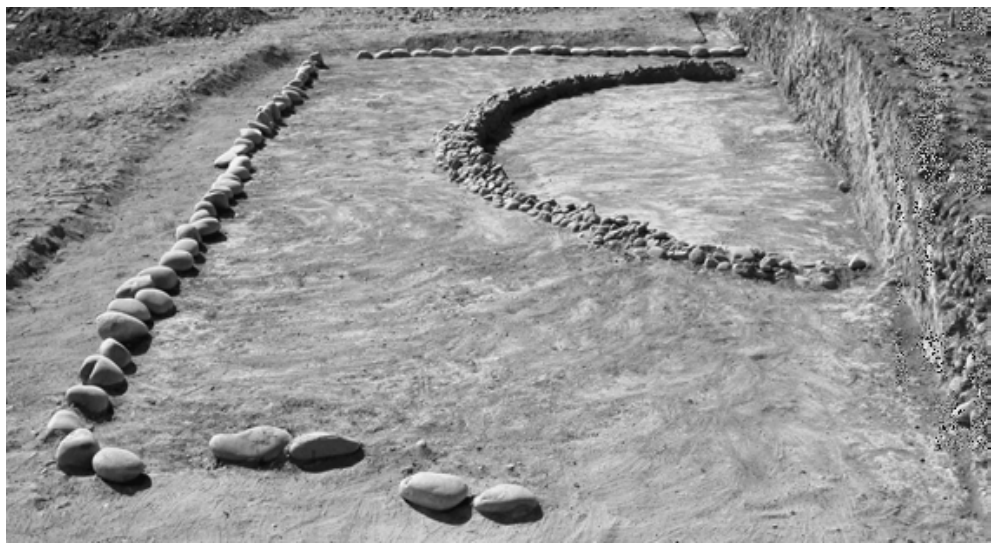

Abb. 48 | Gf. Žoan Tobe, Kurgan 8.

Planum. Osthälfte. Blick von N

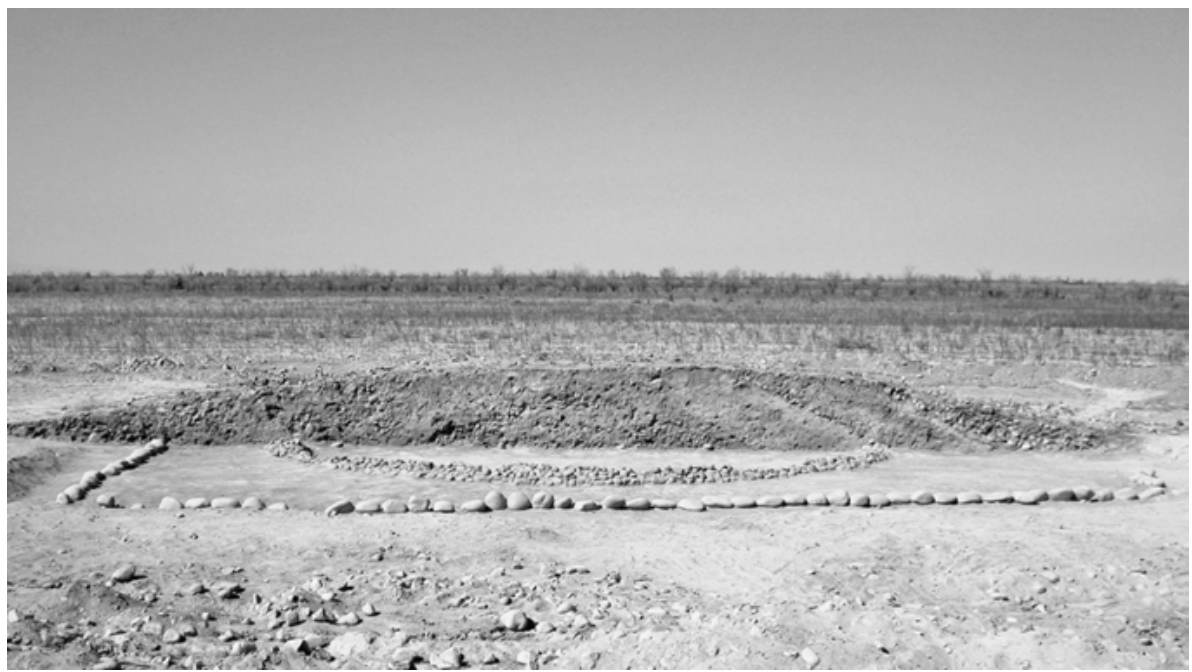

Abb. 49 | Gf. Žoan Tobe, Kurgan 8. Planum und Profil I. Blick von O

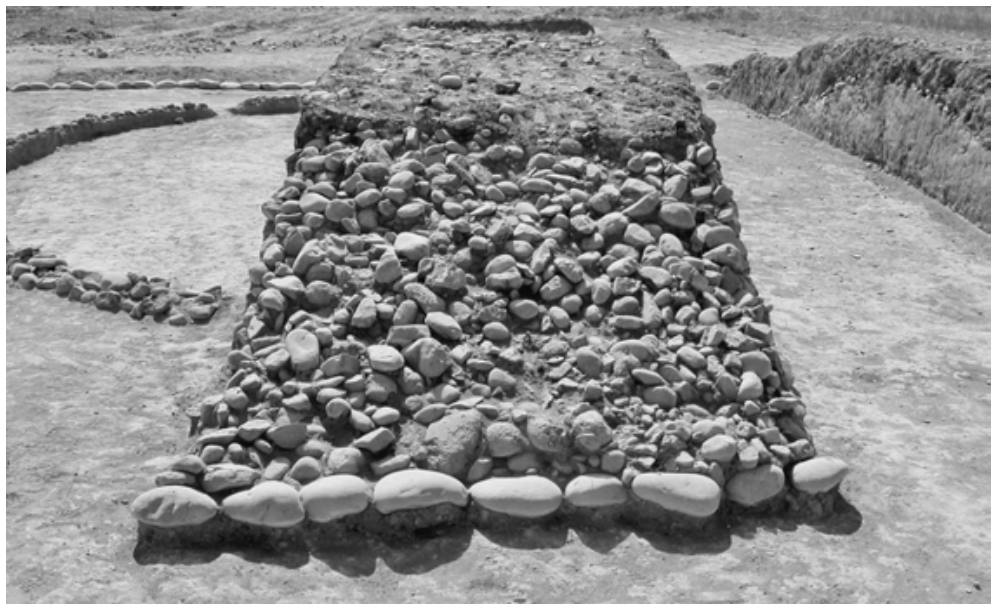

Abb. 50 | Gf. Žoan Tobe, Kurgan 8.

Fragment des Steinpanzesr. Blick von N 
Im Bereich der Kurgankuppe konnten weder eine äußere dünnflüssige Lehmversiegelung noch die Steinabdeckung des Kurgans festgestellt werden. Als ein einfaches Spezifikum der Baukonstruktion darf es nicht erklärt werden, da die ganze Oberfläche des Grabhügels intensiv durchgepflügt worden ist. Möglicherweise wurden diese Schichten durch den Pflug zerstört. Anhand der neugewonnenen Kenntnisse konnte das endgültige Aussehen des Kurgans nicht eindeutig rekonstruiert werden.

Dieselbe Aussage gilt auch für Kurgan 9 (Abb. 47).

Der Kurgan 9 wies eine ähnliche Struktur der Aufschüttung wie der Kurgan 8 auf, allerdings mit Unterschieden in den äußeren Formen der Tumuli. Als Randmarkierung dienten hier große und mittlere Steine, die zum äußeren Steinpanzer gehören, jedoch keinen klaren Kranz, Ring oder Ähnliches darstellen.

Die Profile 2 und 5 weisen eine weitgehend identische Situation auf, mit dem Unterschied, dass im Profil 2 mittig ein Raubtrichter zu sehen ist. Der Kurgan wurde mehrmals beraubt und die Spuren dafür befinden sich überwiegend im Zentrum des Kurgans sowie im mittleren Bereich mit leichter östlicher Versetzung (Profile I-4; Abb. 47,I-4 und Abb. 46). An beiden Profilen konnte ein mittlerer Kern aus sandhaltigem feinem Lehm festgestellt werden, der mit einer dünnen Schicht (8-20 mm) dünnflüssigen Lehms verschmiert wurde. Darauf wurde im gleichen Bauabschnitt noch eine Schicht aus mittelgroßen Steinen gesetzt. Es folgte eine Schicht harten, zum Teil gestampften Lehms mit Sand- und Kiesanteilen, die dem Kurgan sein endgültiges Aussehnen verlieh. Diese Schicht maß im Zentrum des Kurgans $0,2-0,4 \mathrm{~m}$ und am Kurganrand I,I-I,3 m. Als nächster Schritt folgte eine weitere Versiegelung des Kurgans mit verschmiertem dünnflüssigem Lehm, auf den wiederum die mittelgroßen Steine gesetzt wurden. Diese Steinabdeckung wurde durch kleinere Steine verstärkt, besonders am Kurganrand.

Im Grunde genommen unterscheiden sich die Profile I und 6 vom Kurgan 9 nicht wesentlich voneinander (Abb. 47,I.6). Beide zeigen eine äußere lehmhaltige Schicht, die durch eine dünnflüssige Lehmschicht bedeckt wurde. Auf diesem dünnflüssigen Lehm wurden die mittelgroßen Steine ausgelegt, die wiederum durch kleinere Steine verstärkt wurden, so dass ein Steinpanzer entstand. Der Unterschied beider Profile liegt darin, dass beim Profil I die Versiegelung des Kurgans, die aus dem nassverschmierten Lehm bestand, durch das ganze Profil läuft. Es ist vorstellbar, dass derartige Lehmverschmierungen den ganzen Kurgan überzogen und darauf gesetzte Steine lückenlos den Kurgan überdeckten. Ob es tatsächlich so war, lässt sich nicht mehr rekonstruieren, da die Oberfläche des Kurgans stark durch die landwirtschaftlichen Aktivitäten moderner Zeit beschädigt ist.

Auf dem Hochplateau Kegen sind einige Kurgane so beschädigt, dass der innere Aufbau sichtbar wurde. So zum Beispiel bestand Kurgan I8213 aus einer dunklen, feinlehmigen Halbkugel, die mit einer helleren Lehmschicht bedeckt worden war (Abb. 5I). Das Ganze verlieh dem Tumulus eine abgerundete, plattformähnliche Form mit abgeflachter Kuppe. Steine fehlten komplett und die äußere Schicht wurde so angelegt, dass der Kurgan drei steile und einen sanften Abhang aufwies. Direkt auf Kurgan I des Plateaus ${ }^{214}$ befindet sich ein durch eine Baumaschine angelegter Weg vom Kurganrand bis zur Kurgankuppe. Die dabei entstandenen Aufschlüsse zeigen, dass die Kurganaufschüttung aus gestampften, mit Lehmmörtel zusammengefügten Lehmziegeln $(20 \times 40 \mathrm{~cm}$ und $20 \times 10 \mathrm{~cm})$ bestand (Abb. 52).

Die Kleinkurgane, die noch als einfache abgerundete Erd- oder Steinerhebungen bzw. Steinkreise sichtbar waren, bestanden überwiegend aus nur zwei Schichten - nämlich aus der oberen Steinpackung und der unteren sandig-lehmigen Schicht bzw. der Lössschicht215.

2I3 Siehe Katalog, Gf. auf dem Plateau Kegen, Kurgancharakteristik.

2I4 Siehe Anm. 213.
2I5 Zum Beispiel: Kleinkurgane vom Gf. Issyk oder vom Gf. Novoalekseevka (siehe Katalog, Gf. Issyk, Forschungsgeschichte und Gf. Novoalekseevka, Forschungsgeschichte.). 


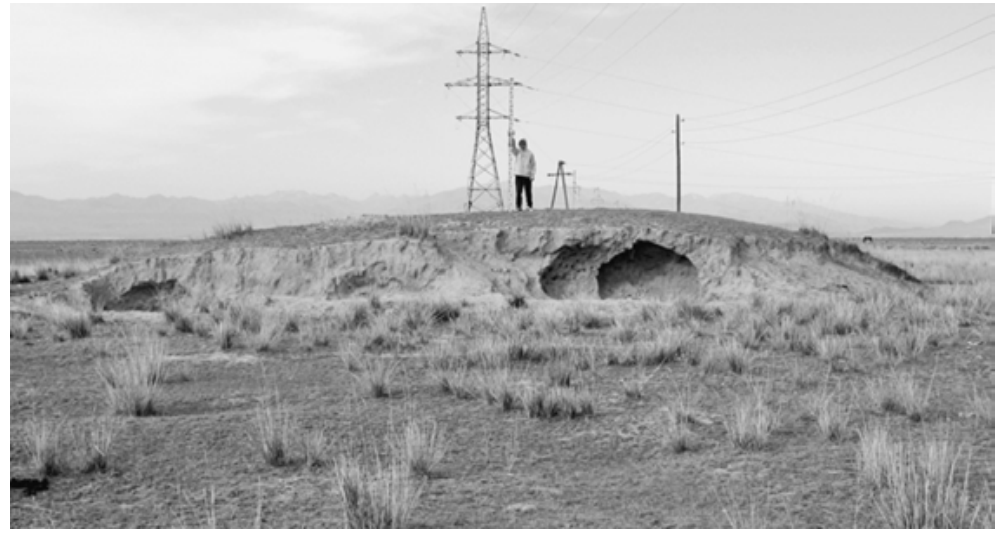

Abb. 51 | Plateau Kegen, Kurgan I8. Blick von $\mathrm{O}$

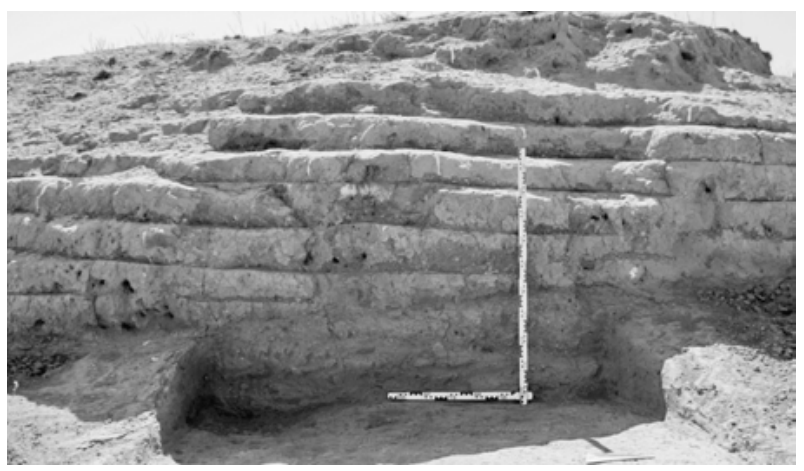

Abb. 52 | Plateau Kegen, Kurgan I. Aufschluss des Kurganaufschüttung, Lehmziegel. Blick von O

Die Großkurgane enthielten in der Regel im Zentrum eine rechteckige Grabgrube mit abgerundeten Ecken (Gf. Issyk, Kurgan № 6; Gf. Turgen, K7; Gf. Ulžan, K2) oder eine quadratische (Gf. Issyk „Kurgan beim Dorf Issyk“). Unter dem „Kurgan Issyk“ befanden sich in der Mitte ein Zentralgrab, das völlig zerstört und komplett beraubt war, sowie ein südlich davon gelegenes Nebengrab mit einer Grabkammer aus Lärchenbalken. Dort lag der. „Goldene Mann von Issyk“. Die Grabgruben der Kurgane 2, 3 und 5 vom Gräberfeld Ulžan wurden mit Halbbohlen oder mit Brettern bedeckt. Im „Kurgan am Steilhang des Turgen-Ufers“ (Gf. Turgen) kamen eine Grabkammer mit Überresten einer undefinierbaren Holzkonstruktion und eine Nachbestattung in einer Grabgrube mit Absätzen zum Vorschein.

Die Gräber waren west-östlich ausgerichtet. Nur im Kurgan 2 des Gräberfeldes Ulžan hatte das Grab eine Nordwest-Südost-Ausrichtung. Die Grabgruben waren 3,I $\mathrm{m}$ bis $5 \mathrm{~m}$ lang, I, $9 \mathrm{~m}$ bis $5 \mathrm{~m}$ breit und I $\mathrm{m}$ bis 2,8 $\mathrm{m}$ tief. In der überwiegenden Zahl handelte es sich um Einzelbestattungen. Nur im Grab des „Kurgans beim Dorf Issyk“ (Gf. Issyk) fand sich eine Doppelbestattung. Fast alle bestatteten Personen erreichten das Erwachsenenalter. Eine Ausnahme stellt der „Goldene Mann von Issyk“ dar, der im juvenilen Alter starb. Anthropologische Geschlechtsbestimmungen fehlen fast vollständigª. Die Toten ruhten in einer Rückenstreckerlage mit den Köpfen nach Westen bzw. Südwesten.

2I6 Nur der „Goldene Mann von Issyk“ wurde von O. I. Ismagulov als ein männliches Individuum bestimmt (Akišev K. A. 1978,62 ). 
Abb. 53 | Silberschale mit einer runenartigen Inschrift, Gf. Issyk „Kurgan Issyk“ (Akišev K. A. I978, Abb. 7I)

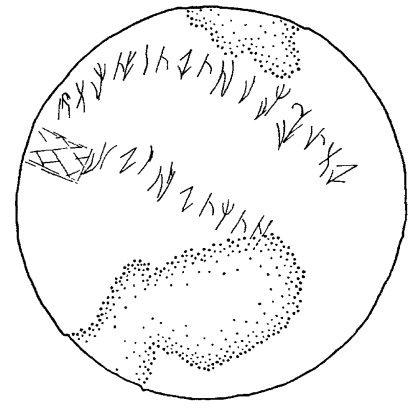

Zum Beigabenrepertoire gehören Waffen, wie z.B. Dolche/ Akinakai²17 und Schwerter aus Eisen mit goldenen Inkrustationen ${ }^{218}$, Pfeilspitzen ${ }^{219}$ und Messer aus Eisen ${ }^{220}$. Weiterhin fand man in solchen Gräbern Trachtgegenstände und Schmuck, eine Reitpeitsche ${ }^{221}$ mit einem von Golddraht umwickelten Knauf, einen Spiegel aus Bronze ${ }^{222}$, eine eiserne Nadel ${ }^{223}$, die in ein Goldblatt eingewickelt war, ein Halsreif aus Gold 224 , goldene Fingerringe 225 und ein Ohrring, ebenfalls aus Gold und mit Türkisperlen besetzt $^{22}$. Zusätzlich wurden den Verstorbenen zahlreiche Gefäße und unterschiedliche Behälter aus verschiedenen Materialien, wie Ton, Holz, Bronze und Silber beigegeben ${ }^{227}$. Besonders hervorzuheben ist eine Silberschale mit einer runenartigen Inschrift (Abb. 53) aus dem „Kurgan Issyk“ (Gf. Issyk), die im Grab des „Goldenen Mannes“ geborgen wurde. Diese Inschrift gilt als bisher ältestes sakisches Schriftzeugnis (Akišev K. A. I978, 57-60, Abb. 70-71; Parzinger 2006, 660).

Die Verstorbenen der sakischen Elite waren in prachtvollen Kleidern bestattet. So trug zum Beispiel der „Goldene Mann von Issyk“228 „eine hohe, spitz zulaufende Kopfbedeckung, eine gegürtete Jacke mit langen Ärmeln, eine Hose sowie halbhohe Stiefel. Haube, Jacke und Stiefel bestanden aus rotem Stoff oder waren mit rotem Stoff überzogen sowie mit fast 4000 aufgenähten Goldblechstücken verziert, die verschiedene figurale Muster wiedergeben“ (Parzinger 2006, 660). Die „Vergoldung“ der Kleider mit Goldblechen oder gegossenen Goldappliken besitzt viele Analogien in den Fürstengräbern der Früheisenzeit im Verbreitungsgebiet des skytho-sakischen Kulturkreises. Parallelen dazu finden sich im Kurgan I des Gräberfeldes Čilikty-2 in Ostkasachstan (Samašev u. a. 2004b, I46-I57), im Fürstengrab 5 des Kurgans Aržan 2 in Tuva, Russische Föderation (Pozdnjakov 2010, Abb. 225-226) oder im Haupt- und Nebengrab des Kurgans Tolstaja Mogila (Mozolevs'kij 1979) bzw. in der Bestattung 2 des Kurgans Soboleva Mogila im mittleren Dnepr-Gebiet, Ukraine (Mozolevskij/Polin 2005, I55-I62, Abb. 9I-I05, Tab. I3-I9).

Die meisten untersuchten Kleinkurgane 229 wiesen im Zentrum eine rechteckige Grabgrube auf ${ }^{230}$. In drei Kurganen wurden zwei parallel zueinander liegende Grabgruben vorgefunden (Gf. Is-

2I7 Siehe Katalog, Gf. Issyk, Forschungsgeschichte, Kurgan "Issyk“.

2I8 Siehe Katalog, Gf. Issyk, Forschungsgeschichte, Kurgan "Issyk“ oder „Kurgan beim Dorf Issyk“.

2I9 Siehe Katalog, Gf. Issyk, Forschungsgeschichte, „Kurgan beim Dorf Issyk“.

220 Siehe Anm. 219.

22I Siehe Anm. 2I7.

222 Siehe Anm. 2I7.

223 Siehe Anm. 219.

224 Siehe Anm. 2I7.
225 Siehe Anm. 2I7.

226 Siehe Anm. 2I7.

227 Siehe Katalog, Gf. Issyk, Forschungsgeschichte, Kurgan "Issyk“ und „Kurgan beim Dorf Issyk“ oder Gf. Turgen, Kurgancharakteristik, Kurgan 7.

228 Siehe Anm. 2I7.

229 Gf. Issyk, Gf. Novoalekseevka und Gf. Žoan Tobe (siehe Katalog).

230 Z. B.: Kleinkurgan 3 des Gf. Issyk (siehe Katalog, Gf. Issyk, Forschungsgeschichte, Auflistung der untersuchten Kurgane [Čarikov ig6o]). 


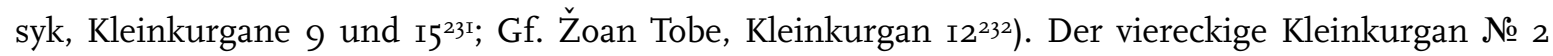
des Gräberfeldes Novoalekseevka enthielt im Zentrum eine Grabgrube, eine Feuerstelle im nordöstlichen Bereich und zwei Nachbestattungen ohne erkennbare Grabgruben in der nordöstlichen Ecke des Kurgans ${ }^{233}$. Drei Kurgane beinhalteten gar keine Grabgruben unter der Kurganaufschüttung (Gf. Issyk, Kleinkurgan 4234; Gf. Novoalekseevka, Kleinkurgane № 4 und № $7^{235}$ ). Neben rechteckigen Grabgruben fand man in Kleinkurganen auch rechteckige Grabgruben mit abgerundeten Ecken² mit einer Nische ${ }^{237}$, mit abgerundeten Ecken und mit Absätzen ${ }^{238}$. Zudem kamen auch abgerundete $^{239}$ bzw. länglich-ovale Grabgruben ${ }^{240}$ zum Vorschein. Die meisten Gruben wurden in der Mitte des Kurgans ${ }^{24 \mathrm{I}}$ ausgehoben. Alternativ befanden sie sich in der Nord-242, Süd-243, Ost-244 oder Westhälfte ${ }^{245}$ sowie im nordöstlichen ${ }^{246}$, südöstlichen ${ }^{247}$ oder südwestlichen Bereich ${ }^{248}$ des Kurgans, am häufigsten jedoch in der Osthälfte. Einige Gräber wurden mit querliegenden Holzbalken bzw. Holzbrettern abgedeckt ${ }^{249}$. Auf dem Grabboden des Kleinkurgans 34 (6) des Gräberfeldes Žoan Tobe befand sich sogar ein gezimmerter Holzrahmen²5. Mehr als die Hälfte aller Gräber wurde westöstlich

23I Siehe Katalog, Gf. Issyk, Forschungsgeschichte, Auflistung der untersuchten Kurgane (Čarikov i960).

232 Siehe Katalog, Gf. Žoan Tobe, Forschungsgeschichte; vgl. Maksimova ig6oa, 6o-6I, Abb. II.

233 Siehe Katalog, Gf. Novoalekseevka, Forschungsgeschichte, Auflistung der untersuchten Kurgane (Kopylov 1956).

234 Siehe Katalog, Gf. Issyk, Forschungsgeschichte, Auflistung der untersuchten Kurgane (Čarikov i960).

235 Siehe Katalog, Gf. Novoalekseevka, Forschungsgeschichte, Auflistung der untersuchten Kurgane (Kopylov I956).

236 Gf. Issyk, Kleinkurgan № 5 (siehe Katalog, Gf. Issyk, Forschungsgeschichte, Auflistung der untersuchten Kurgane [Bajpakov u.a. 2005c]).

237 Gf. Novoalekseevka, Kleinkurgane № I und № 2 (siehe Katalog, Gf. Novoalekseevka, Forschungsgeschichte, Auflistung der untersuchten Kurgane [Kopylov 1956]).

238 Gf. Žoan Tobe, K8 (siehe Katalog, Gf. Žoan Tobe, Kurgancharakteristik).

239 Gf. Issyk, Kleinkurgan I5, Grab I (siehe Katalog, Gf. Issyk, Forschungsgeschichte, Auflistung der untersuchten Kurgane [Carikov ig6o]).

240 z. B.: Gf. Žoan Tobe, Kleinkurgan io (siehe Katalog, Gf. Žoan Tobe, Forschungsgeschichte; vgl. Maksimova I96oa, 62, Abb. I2,9).

24I 28 Kleinkurgane: Gf. Issyk, Kleinkurgane I-3, 7, I8-I9, 25, 3I, 35 (siehe Katalog, Gf. Issyk, Forschungsgeschichte, Auflistung der untersuchten Kurgane [Čarikov I96o]) und Kleinkurgan № 5 (siehe Katalog, Gf. Issyk, Forschungsgeschichte, Auflistung der untersuchten Kurgane [Bajpakov u.a. 2005c]); Gf. Novoalekseevka, Kleinkurgane № I und № 2 (siehe Katalog, Gf. Novoalekseevka, Forschungsgeschichte, Auflistung der untersuchten Kurgane [Kopylov I956]); Gf. Žoan Tobe, K8-9 (siehe Katalog, Gf. Žoan Tobe, Kurgancharakteristik), Kleinkurgane 3I (5), 34 (6), 36 (9) (siehe Katalog, Gf. Žoan Tobe, Forschungsgeschichte; vgl. Čarikov ${ }_{195} 6 \mathrm{a}$, 29-36), Kleinkurgan 8 (siehe Katalog, Gf. Žoan Tobe, Forschungsgeschichte; vgl. Maksimova I956a, 3-8), Kleinkurgane 5 (77), I (43), 2 (44), 3 (62), 94 (7), 6I (siehe Katalog, Gf. Žoan Tobe, Forschungsgeschichte; vgl. Čarikov I956b, 27-29, 33-40, 47-52), und Kleinkurgane I (Plan № 5), 4, 6 und I8 (siehe Katalog, Gf. Žoan Tobe, Forschungsgeschichte; vgl. Pacevič I956, 33-38).

242 Gf. Žoan Tobe, Kleinkurgan I2, Grab I (siehe Katalog, Gf. Žoan Tobe, Forschungsgeschichte; vgl. Maksimova I960a, 6o-6I, Abb. II).

243 Gf. Issyk, Kleinkurgane I2 und I6 (siehe Katalog, Gf. Issyk, Forschungsgeschichte, Auflistung der untersuchten Kurgane [Čarikov I960]) und Gf. Žoan Tobe, Kleinkurgan I2, Grab 2 (siehe Katalog, Gf. Žoan Tobe, Forschungsgeschichte; vgl. Maksimova I96oa, 60-6I, Abb. II).

244 Gf. Issyk, Kleinkurgan I5, Grab 2; Kleinkurgane 20 und 29 (siehe Katalog, Gf. Issyk, Forschungsgeschichte, Auflistung der untersuchten Kurgane [Čarikov 1960]) und Gf. Žoan Tobe, Kleinkurgane 4 (48), 6 (78), 84 (5), 8I (siehe Katalog, Gf. Žoan Tobe, Forschungsgeschichte; vgl. Carikov I956b, 30-32, 4I-46, 53-55).

245 Gf. Issyk, Kleinkurgane I3 und I5, Grab I und Kleinkurgan I7 (siehe Katalog, Gf. Issyk, Forschungsgeschichte, Auflistung der untersuchten Kurgane [Čarikov I96o]).

246 Gf. Issyk, Kleinkurgan 9, Grab 2 (siehe Katalog, Gf. Issyk, Forschungsgeschichte, Auflistung der untersuchten Kurgane [Čarikov ig60]).

247 Gf. Issyk, Kleinkurgan 8 (siehe Katalog, Gf. Issyk, Forschungsgeschichte, Auflistung der untersuchten Kurgane [Carikov i960]).

248 Gf. Issyk, Kleinkurgan 9, Grab I und Kleinkurgane 27 und 30 (siehe Katalog, Gf. Issyk, Forschungsgeschichte, Auflistung der untersuchten Kurgane [Čarikov i960]).

249 Sieben Gräber: Gf. Žoan Tobe, Kleinkurgan 3I [5] (siehe Katalog, Gf. Žoan Tobe, Forschungsgeschichte; vgl. Čarikov I956a, 29-3I), Kleinkurgan I2, Gräber I und 2, Kleinkurgane to und 70 (siehe Katalog, Gf. Žoan Tobe, Forschungsgeschichte; vgl. Maksimova I96oa, 60-62, Abb. II-I2,2. 5. 9-IO), Kleinkurgan I [43] (siehe Katalog, Gf. Žoan Tobe, Forschungsgeschichte; vgl. Čarikov I956b, 33-35), und Kleinkurgan 4 (siehe Katalog, Gf. Žoan Tobe, Forschungsgeschichte; vgl. Pacevič 1956 , 36-37).

250 Siehe Katalog, Gf. Žoan Tobe, Forschungsgeschichte; vgl. Čarikov I956a, 32-33. 
ausgerichtet ${ }^{251}$. Zu gleichen Teilen wurden zudem Nord-Süd-252 und Nordwest-Südost-Ausrichtungen 253 nachgewiesen. Die Grabgruben waren I,6 $\mathrm{m}$ bis 5,3 $\mathrm{m}$ lang, 0,5 $\mathrm{m}$ bis 2,75 $\mathrm{m}$ breit und 0,4 bis I,6 $\mathrm{m}$ tief ${ }^{254}$. Fast ausschließlich ruhten in diesen Gräbern einzeln bestattete, erwachsene Individuen255. Nur in zwei Gräbern (Kleinkurgane I (nach Plan K5) und 8I des Gräberfeldes Žoan Tobe) wurde je eine Doppelbestattung ${ }^{25} 6$ festgestellt. In beiden Fällen handelte es sich um eine erwachsene Person und um ein Kind ${ }^{257}$. In vier Fällen lagen nur Kinder in den Gräbern ${ }^{25}{ }^{8}$. Eine separate Pferdebestattung ist ebenso dokumentiert (Gf. Žoan Tobe, Kleinkurgan I2)259. Mit einer Ausnahme waren die Bestatteten in Rückenstreckerposition bestattet. Nur ein einziges Individuum (Gf. Žoan Tobe, Kleinkurgan 94 [7]) lag in Rückenhockerlage260.

Die Gräber wurden überwiegend ärmlich ausgestattet. Zum Beigabenrepertoire ${ }^{26 \mathrm{I}}$ gehören Messer $^{262}$, Ohrringe ${ }^{263}$, Besatzstücke ${ }^{264}$, Riemenverteiler ${ }^{265}$, Steigbügelschnallen ${ }^{266}$ und Trensen ${ }^{267}$ aus Bronze, Keramikscherben ${ }^{268}$, Krüge ${ }^{269}$ und Schalen ${ }^{270}$, Perlen aus Aragonit ${ }^{271}$ oder Glaspaste ${ }^{272}$ bzw. Pyrit273, Astragale274, Tierknochen ${ }^{275}$ (Schaf/Ziege oder Pferd), Ockerklumpen ${ }^{276}$, Holzfragmente ${ }^{277}$ oder Holzkohle278. Einmalig kamen ein Goldplättchen²79, eine Goldhülse ${ }^{280}$, ein Pfriem² ${ }^{281}$ aus Bronze, eine eiserne Haarnadel, die in einem Goldblatt eingewickelt worden war ${ }^{282}$, eine einfache Haarnadel 283 , eine Pfeilspitze ${ }^{284}$ und ein Messer aus Eisen"285, ein $\mathrm{Napf}^{286}$, eine steinerne Handmühle ${ }^{287}$, ein Spinn-

25I 29 Gräber, z. B.: Gf. Issyk, Kleinkurgane I und 2 (siehe Katalog, Gf. Issyk, Forschungsgeschichte, Auflistung der untersuchten Kurgane [Čarikov i960]) oder Kleinkurgan № 5 (siehe Katalog, Gf. Issyk, Forschungsgeschichte, Auflistung der untersuchten Kurgane [Bajpakov u.a. 2005 c]).

252 Zehn Gräber: Gf. Issyk Kleinkurgane 3, 7-8, I2, I5 Grab 2, I6, I8, 20, 25 und 30 (siehe Katalog, Gf. Issyk, Forschungsgeschichte, Auflistung der untersuchten Kurgane [Črikov ig6o]).

253 Zehn Gräber: Gf. Issyk, Kleinkurgan 9, Gräber I und 2, Kleinkurgane I7, I9, 27, 29, 3I und 35 (siehe Katalog, Gf. Issyk, Forschungsgeschichte, Auflistung der untersuchten Kurgane [Čarikov I96o]); Gf. Žoan Tobe, Kleinkurgan 36 [9] (siehe Katalog, Gf. Žoan Tobe, Forschungsgeschichte; vgl. Čarikov I956a, 34-36) und Kleinkurgan i8 (siehe Katalog, Gf. Žoan Tobe, Forschungsgeschichte; vgl. Pacevič $1956,37-38$ ).

254 Siehe im Katalog Forschungsgeschichte der Gf. Issyk, Gf. Novoalekseevka und Gf. Žoan Tobe.

25520 Gräber, z. B.: Gf. Novoalekseevka, Kleinkurgan № I (siehe Katalog, Gf. Novoalekseevka, Forschungsgeschichte, Auflistung der untersuchten Kurgane [Kopylov I956]).

256 Siehe Katalog, Gf. Žoan Tobe, Forschungsgeschichte; vgl. Čarikov i956b, 53-55, Pacevič 1956, 33.

257 Die anthropologische Bestimmung stammt vom Ausgräber: Kleinkurgan I (Plan K5) - G. I. Pacevič und Kleinkurgan 8I - A. A. Čarikov.

258 Gf. Issyk, Kleinkurgan I (siehe Katalog, Gf. Issyk, Forschungsgeschichte, Auflistung der untersuchten Kurgane [Čarikov ig6o]); Gf. Novoalekseevka Kleinkurgan № 2, Nachbestattungen I und 2 (siehe Katalog, Gf. Novoalekseevka, Forschungsgeschichte, Auflistung der untersuchten Kurgane [Kopylov I956]); Gf. Žoan Tobe, Kleinkurgan 4 (siehe Katalog, Gf. Žoan Tobe, Forschungsgeschichte; vgl. Pacevič I956, 36).
259 Siehe Katalog, Gf. Žoan Tobe, Forschungsgeschichte; vgl. Maksimova ı96oa, 6o-6ı, Abb. II.

260 Siehe Katalog, Gf. Žoan Tobe, Forschungsgeschichte; vgl. Čarikov I956b, 47-49.

26I Siehe im Katalog Forschungsgeschichte der Gf. Issyk, Gf. Novoalekseevka und Gf. Žoan Tobe.

262 Gf. Žoan Tobe, Kleinkurgane 4 (48) und I2.

263 Gf. Žoan Tobe, Kleinkurgane 5 (77) und 6 (78).

264 Gf. Žoan Tobe, Kleinkurgan I2.

265 Siehe Anm. 264.

266 Siehe Anm. 264.

267 Siehe Anm. 264.

268 Gf. Issyk, Kleinkurgane 2 und № 5; Gf. Novoalekseevka, Kleinkurgane № I-2 und № 4; Gf. Žoan Tobe, Kleinkurgane 6 (78), 8, Іо, $34(6), 84$ (5).

269 Gf. Žoan Tobe, Kleinkurgane 5 (77) und 70.

270 Gf. Žoan Tobe, Kleinkurgane 8 und 49.

27 I Gf. Žoan Tobe, Kleinkurgane 4 (48) und Io.

272 Gf. Žoan Tobe, Kleinkurgane I (43) und Io.

273 Gf. Žoan Tobe, Kleinkurgan 4 (48).

274 Gf. Novoalekseevka, Kleinkurgan № I.

275 Gf. Novoalekseevka, Kleinkurgan № I; Gf. Žoan Tobe, Kleinkurgane 5 (77), 8, 36 (9), 6r und 70.

276 Gf. Žoan Tobe, Kleinkurgan I (43).

277 Gf. Issyk, Kleinkurgan № 5; Gf. Žoan Tobe, Kleinkurgane I (43), 2 (44), 3 (62), 4 (48), 5 (77), 6 (78), 36 (9), 6 г.

278 Gf. Issyk, Kleinkurgan 8; Gf. Novoalekseevka, Kleinkurgan № 2.

279 Gf. Žoan Tobe, Kleinkurgan 8.

280 Siehe Anm. 279.

28I Gf. Žoan Tobe, Kleinkurgan 5 (77).

282 Gf. Žoan Tobe, Kleinkurgan 84 (5).

283 Gf. Žoan Tobe, Kleinkurgan 84 (5).

284 Gf. Žoan Tobe, Kleinkurgan 9.

285 Siehe Anm. 284.

286 Siehe Anm. 279.

287 Gf. Issyk, Kleinkurgan 3I. 
wirtel aus Sandstein ${ }^{288}$, ein trapezförmiger Kalkstein ${ }^{289}$, ein Kamm aus Knochen ${ }^{290}$, ein weiterer aus Holz $^{291}$ sowie eine Plastik aus Knochen (Pferdekopf ?) ${ }^{292}$ zum Vorschein.

\subsubsection{Peripherie der Kurgane}

Am Rande von 73 Großkurganen (23\% aller untersuchten Großkurgane) und von 35 Kleinkurganen (6\% aller untersuchten Kleinkurgane), was insgesamt is \% aller untersuchten Kurgane ausmacht (Iо8 Grabhügel), wurden in der Peripherie noch weitere Konstruktionen festgestellt. Es handelt sich hier um sog. Prozessionswege 293 (bei I9 Kurganen), Kreisgräben²94 (bei 28 Kurganen), Steinkreise 295 (bei 35 Kurganen) oder um Sonderfälle ${ }^{29}$, wie Wälle (bei zehn Kurganen), Plattformen (bei zwei Kurganen) oder Baurampen (bei einem Kurgan). Weitere Befunde werden im Folgenden als „Peripheriekonstruktionen“ bezeichnet. Sie treten im Umfeld von 22 Großkurganen auf. Dazu zählen kleinere, abgerundete, steinerne Konstruktionen, die einige wenige Meter vom Rand des Kurgans liegen und die man als Kleinkurgane ansprechen kann. Außerdem wurden steinkistenähnliche Konstruktionen dokumentiert. Ferner befanden sich im Randbereich der Kurgane einzelne Befunde, die man nicht mehr eindeutig interpretieren kann. Alle Peripheriekonstruktionen lagen, falls noch weitere Konstruktionselemente wie sog. Prozessionswege, Kreisgräben oder Steinkreise vorkamen, zwischen diesen und dem Kurganrand 297.

Im Allgemeinen lässt sich noch anmerken, dass sich die überwiegende Mehrheit aller Kurgane heute auf modernen Ackerflächen befindet und die Peripherie der Grabanlagen durch den Pflug komplett zerstört oder stark beschädigt ist.

\subsubsection{Peripheriekonstruktionen}

Im Randbereich von 22 Kurganen 298 , auf insgesamt elf untersuchten Gräberfeldern wurden unterschiedliche Peripheriekonstruktionen (Tabelle I6; Karte I2) festgestellt (Abb. 54-55). Diese Bauten finden sich neben Kurganen aller drei Größenkategorien, mit einer Ausnahme (Gf. Aksaj-9, Kıo²99: H=I,5 m; $\mathrm{Dm}=\mathbf{2 2} \mathrm{m})$. Fast die Hälfte solcher Tumuli mit angrenzenden Konstruktionen sind große Kurgane (2-4 m) (zehn Grabhügel; 48\% aller Kurgane mit Peripheriekonstruktionen). Etwas seltener finden sich solche Befunde neben größeren Kurganen (4-8 m) (acht Grabhügeln; $38 \%$ ). In drei Fällen (Höhe über 8 m) (I4\%; Gf. Asy Saga, KI ${ }^{300}$, Gf. Turgen, K3 $3^{301}$ und Gf. Žoan Tobe KI ${ }^{302}$ ) handelt es sich um sehr große Kurgane, deren Peripherie mit mehreren Peripheriekonstruktionen bebaut worden war. Die Kurgane, de-

288 Gf. Žoan Tobe, Kleinkurgan I (43).

289 Gf. Žoan Tobe, Kleinkurgan 5 (77).

290 Siehe Anm. 264.

29I Siehe Anm. 273.

292 Siehe Anm. 264.

293 Dazu siehe Kap. 5.I.5.2 Sog. Prozessionswege.

294 Dazu siehe Kap. 5.I.5.3 Kreisgräben.

295 Dazu siehe Kap. 5.I.5.4 Steinkreise.

296 Dazu siehe Kap. 5.I.5.5 Sonderfälle.

297 Selbstverständlich befinden sich sog. Prozessionswege, Kreisgräben und Steinkreise auch in der Peripherie der Kurgane. Um jedoch solche architektonischen Elemente voneinander $\mathrm{zu}$ unterscheiden, werden in der Arbeit Kleinkurgane und steinkistenähnliche Konstruktionen als „Peripheriekonstruktionen“, und weitere Befunde als „Prozessionsweg“, „Kreisgraben“, „Steinkreis“ oder "Sonderfälle“ bezeichnet.

298 Ein Kurgan 5 km südwestlich vom Dorf Žalauly; Gf. Aksaj-9, K8 und Kıo; Gf. Aktasty-3, K4; Gf. Asy Saga, KI und K4; Gf. Boroldaj, Kı6; Gf. Issyk, K9, K2I-22 und K30-3I; Gf. Kyzylšaryk, KiI und KI4-I5; Gf. Turgen, K3 und K4; Gf. Žalauly-2, K6I; Gf. Žoan Tobe, KI; Gf. Žylysaj-I, K6-7 und K9.

299 Siehe Katalog, Gf. Aksaj-9, Kurgancharakteristik.

300 Siehe Katalog, Gf. Asy Saga, Kurgancharakteristik.

30I Siehe Katalog, Gf. Turgen, Kurgancharakteristik.

302 Siehe Katalog, Gf. Žoan Tobe, Kurgancharakteristik. 
Tabelle 16 | Kurgane mit Peripheriekonstruktionen (Peripheriekonstruktionen grau markiert)

\begin{tabular}{|c|c|c|c|c|c|c|c|c|c|c|c|c|}
\hline$\frac{\overrightarrow{0}}{8}$ & 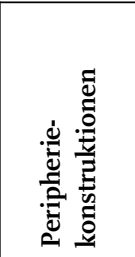 & 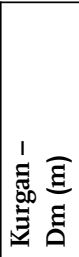 & 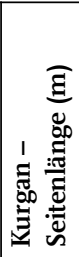 & 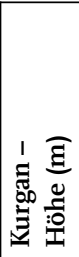 & 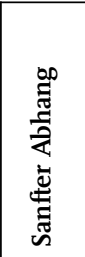 & 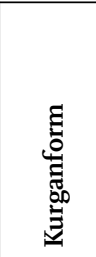 & 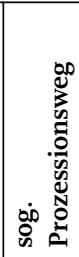 & 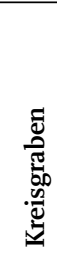 & 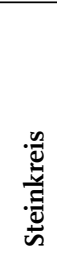 & 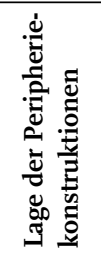 & 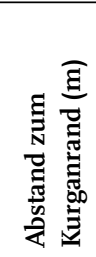 & 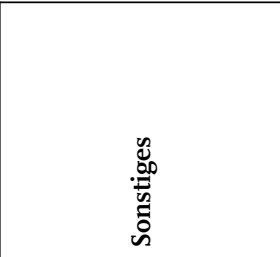 \\
\hline \multirow{2}{*}{$\begin{array}{l}\text { Ein Kurgan } \\
5 \mathrm{~km} \text { süd- } \\
\text { westlich } \\
\text { vom Dorf } \\
\text { Žalauly }\end{array}$} & $\mathrm{IK}_{5} \mathrm{SW}$ & & 28 & 3 & keine & $\begin{array}{l}\text { vier- } \\
\text { eckig }\end{array}$ & & $\mathrm{x}$ & & NO & $\begin{array}{l}\text { direkt } \\
\text { am }\end{array}$ & $\begin{array}{l}\text { Kleinkurgan innerhalb } \\
\text { des Kreisgrabens }\end{array}$ \\
\hline & $\begin{array}{l}\text { Klein- } \\
\text { kurgan I }\end{array}$ & I4 & & $\mathrm{I}, 2$ & & $\begin{array}{l}\text { abge- } \\
\text { rundet }\end{array}$ & & & & & & \\
\hline \multirow[t]{4}{*}{ Gf. Aksaj-9 } & K8 & $3 \mathrm{I}$ & & 3 & S & $\begin{array}{l}\text { abge- } \\
\text { rundet }\end{array}$ & $\mathrm{x}$ & & & NW & 5 & $\begin{array}{l}\text { Kleinkurgan innerhalb } \\
\text { des Kreisgrabens }\end{array}$ \\
\hline & $\begin{array}{l}\text { K9 (Klein- } \\
\text { kurgan) }\end{array}$ & 5 & & O,I & & $\begin{array}{l}\text { abge- } \\
\text { rundet }\end{array}$ & & & & & & \\
\hline & Kio & 22 & & $\mathrm{I}, 5$ & $S$ & $\begin{array}{l}\text { abge- } \\
\text { rundet }\end{array}$ & & & $\mathrm{x}$ & SW & 0,3 & $\begin{array}{l}\text { Kleinkurgan innerhalb } \\
\text { des Kreisgrabens }\end{array}$ \\
\hline & $\begin{array}{l}\text { KII (Klein- } \\
\text { kurgan) }\end{array}$ & 3 & & $0, \mathrm{I}$ & & $\begin{array}{l}\text { abge- } \\
\text { rundet }\end{array}$ & & & & & & \\
\hline $\begin{array}{l}\text { Gf. } \\
\text { Aktasty-3 }\end{array}$ & $\mathrm{K}_{4}$ & 25 & & 2,5 & $S$ & $\begin{array}{l}\text { abge- } \\
\text { rundet }\end{array}$ & $x$ & & & $\mathrm{~N}$ & 2 & $\begin{array}{l}5 \text { rechteckigen Vertie- } \\
\text { fungen-Bedeutung ??? }\end{array}$ \\
\hline \multirow[t]{2}{*}{$\begin{array}{l}\text { Gf. } \\
\text { Asy Saga }\end{array}$} & KI & I49 & & $2 \mathrm{I}$ & $S$ & $\begin{array}{l}\text { abge- } \\
\text { rundet }\end{array}$ & $x$ & & & $\begin{array}{l}\text { um den } \\
\text { Kurgan }\end{array}$ & 20 & 2I Kleinkurgane \\
\hline & $\mathrm{K}_{4}$ & 62 & & 5 & SSW & $\begin{array}{l}\text { abge- } \\
\text { rundet }\end{array}$ & $\mathrm{x}$ & & & NW & IO & 4 Kleinkurgane \\
\hline \multirow[t]{2}{*}{$\begin{array}{l}\text { Gf. } \\
\text { Boroldaj }\end{array}$} & Ki6 & 150 & & $\mathrm{I} 4,4$ & keine & $\begin{array}{l}\text { abge- } \\
\text { rundet }\end{array}$ & & & & $\mathrm{S}$ & $\begin{array}{l}\text { direkt } \\
\text { am } \\
\text { Rand }\end{array}$ & $\begin{array}{l}\text { in der Peripherie: zwei } \\
\text { Steinkreise, ein Klein- } \\
\text { kurgan, eine Stele }\end{array}$ \\
\hline & $\begin{array}{l}\text { Ki6 } \\
\text { (Klein- } \\
\text { kurgan) }\end{array}$ & 20 & & $?$ & & $\begin{array}{l}\text { abge- } \\
\text { rundet }\end{array}$ & & & & & & \\
\hline \multirow[t]{8}{*}{ Gf. Issyk } & K9 & 46 & & 4 & $\mathrm{O}$ & $\begin{array}{l}\text { abge- } \\
\text { rundet }\end{array}$ & & & & $\mathrm{N}+\mathrm{NW}$ & II & $\begin{array}{l}3 \text { Kreiskonstruktionen } \\
\text { (ethnographische Zeit) }\end{array}$ \\
\hline & zum K9 & IO & & $?$ & & $\begin{array}{l}\text { abge- } \\
\text { rundet }\end{array}$ & & & & & & Kreiskonstruktion \\
\hline & zum K9 & I8 & & $?$ & & $\begin{array}{l}\text { abge- } \\
\text { rundet }\end{array}$ & & & & & & Kreiskonstruktion \\
\hline & zum K9 & I6 & & $?$ & & $\begin{array}{l}\text { abge- } \\
\text { rundet }\end{array}$ & & & & & & Kreiskonstruktion \\
\hline & K2I & 46 & & 4,5 & $\mathrm{SO}$ & $\begin{array}{l}\text { abge- } \\
\text { rundet }\end{array}$ & & & & S & 37 & 2 Kleinkurgane \\
\hline & $\mathrm{K} 22$ & 34 & & 2,5 & $\mathrm{SO}$ & $\begin{array}{l}\text { abge- } \\
\text { rundet }\end{array}$ & & & & S & I2+I9 & 2 Kleinkurgane \\
\hline & zum K22 & 8 & & $?$ & & $\begin{array}{l}\text { abge- } \\
\text { rundet }\end{array}$ & & & & & I2 & \\
\hline & zum K22 & 14 & & $?$ & & $\begin{array}{l}\text { abge- } \\
\text { rundet }\end{array}$ & & & & & I9 & \\
\hline
\end{tabular}




\begin{tabular}{|c|c|c|c|c|c|c|c|c|c|c|c|c|}
\hline 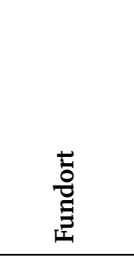 & 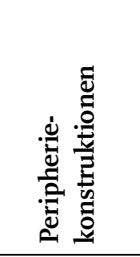 & 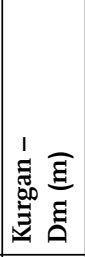 & 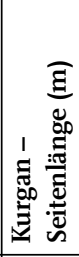 & 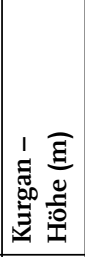 & 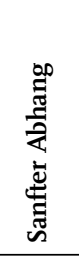 & 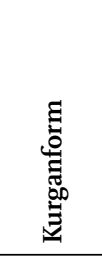 & 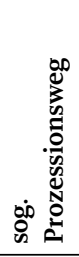 & 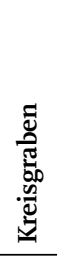 & 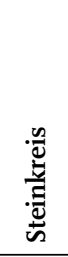 & 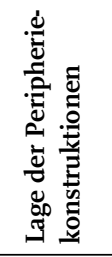 & 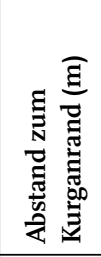 & 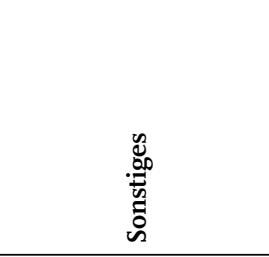 \\
\hline \multirow[t]{4}{*}{ Gf. Issyk } & $\mathrm{K}_{3} \mathrm{O}$ & 58 & & 5,3 & SO & $\begin{array}{l}\text { abge- } \\
\text { rundet }\end{array}$ & & & & $S$ & $\begin{array}{l}\text { direkt } \\
\text { am } \\
\text { Rand }\end{array}$ & I Kleinkurgan \\
\hline & $\begin{array}{l}\text { K30 } \\
\text { (Klein- } \\
\text { kurgan) }\end{array}$ & I4 & & 0,3 & & $\begin{array}{l}\text { abge- } \\
\text { rundet }\end{array}$ & & & & & & \\
\hline & $\mathrm{K}_{3} \mathrm{I}$ & 66 & & 6 & $\mathrm{~S}$ & $\begin{array}{l}\text { abge- } \\
\text { rundet }\end{array}$ & & & & SO & $\begin{array}{l}\text { direkt } \\
\text { am } \\
\text { Rand }\end{array}$ & I Kleinkurgan \\
\hline & $\begin{array}{l}\text { K33 } \\
\text { (Klein- } \\
\text { kurgan) }\end{array}$ & 35 & & ? & SO & $\begin{array}{l}\text { abge- } \\
\text { rundet }\end{array}$ & & & & & & Kuppe abgeflacht \\
\hline \multirow[t]{8}{*}{$\begin{array}{l}\text { Gf. } \\
\text { Kyzylšaryk }\end{array}$} & KII & 37 & & 3 & S & $\begin{array}{l}\text { abge- } \\
\text { rundet }\end{array}$ & & & & & & 5 Steinkonstruktionen \\
\hline & zum KII & $\begin{array}{l}2,9 \mathrm{x}- \\
0,5\end{array}$ & & 0,2 & & $\begin{array}{l}\text { recht- } \\
\text { eckig }\end{array}$ & & & & $\mathrm{O}$ & I5 & N-S Ausrichtung \\
\hline & zum KiI & $\begin{array}{l}2,9 \mathrm{X}- \\
2, \mathrm{I}\end{array}$ & & 0,2 & & $\begin{array}{l}\text { recht- } \\
\text { eckig }\end{array}$ & & & & $\mathrm{O}$ & I2 & W-O Ausrichtung \\
\hline & zum KiI & $\begin{array}{l}2,6 \mathrm{x}- \\
1,5\end{array}$ & & 0,2 & & $\begin{array}{l}\text { recht- } \\
\text { eckig }\end{array}$ & & & & W & I5 & N-S Ausrichtung \\
\hline & zum KiI & $\begin{array}{l}4,3 \mathrm{X}- \\
\mathrm{I}, 4\end{array}$ & & 0,2 & & $\begin{array}{l}\text { recht- } \\
\text { eckig }\end{array}$ & & & & W & I2 & W-O Ausrichtung \\
\hline & zum KiI & $\begin{array}{l}2,8 \mathrm{x}- \\
0,8\end{array}$ & & 0,2 & & $\begin{array}{l}\text { recht- } \\
\text { eckig }\end{array}$ & & & & W & 9 & N-S Ausrichtung \\
\hline & KI4 & 56 & & 7 & S & $\begin{array}{l}\text { abge- } \\
\text { rundet }\end{array}$ & $\mathrm{x}$ & & & $\begin{array}{l}\text { um den } \\
\text { Kurgan }\end{array}$ & 5 & 3 Kleinkurgane \\
\hline & KI5 & 6I & & 7 & S & $\begin{array}{l}\text { abge- } \\
\text { rundet }\end{array}$ & & & & $\mathrm{S}+\mathrm{O}$ & überall & 8 Kleinkurgane \\
\hline \multirow[t]{4}{*}{ Gf.Turgen } & $\mathrm{K}_{3}$ & 70 & & 7 & S & $\begin{array}{l}\text { abge- } \\
\text { rundet }\end{array}$ & $\mathrm{x}$ & & $\mathrm{x}$ & $\begin{array}{l}\text { um den } \\
\text { Kurgan }\end{array}$ & überall & \\
\hline & $\begin{array}{l}\text { 4I Klein- } \\
\text { kurgan } \\
\mathrm{Dm}= \\
\mathrm{I}, 5-6,3 \mathrm{~m}\end{array}$ & & & & & & & & & & & \\
\hline & $\mathrm{K}_{4}$ & $5^{\mathrm{I}}$ & 6,5 & $S$ & $\begin{array}{l}\text { abge- } \\
\text { run- } \\
\text { det }\end{array}$ & $\mathrm{x}$ & & & NO & I2 & $\begin{array}{l}\text { I Klein- } \\
\text { kurgan }\end{array}$ & \\
\hline & $\begin{array}{l}\text { K4-Klein- } \\
\text { kurgan } \\
\text { im O }\end{array}$ & I2 & & 0,2 & & $\begin{array}{l}\text { abge- } \\
\text { rundet }\end{array}$ & & & & & & \\
\hline \multirow[t]{2}{*}{$\begin{array}{l}\text { Gf. } \\
\text { Žalauly-2 }\end{array}$} & K6I & 37 & & 4 & S & $\begin{array}{l}\text { abge- } \\
\text { rundet }\end{array}$ & $\mathrm{x}$ & & & & & 3 Steinkonstruktionen \\
\hline & zum K6I & $4,5 \mathrm{XI}$ & & 0,2 & & $\begin{array}{l}\text { recht- } \\
\text { eckig }\end{array}$ & & & & SO & $\begin{array}{l}\text { direkt } \\
\text { am } \\
\text { Rand }\end{array}$ & W-O Ausrichtung \\
\hline
\end{tabular}




\begin{tabular}{|c|c|c|c|c|c|c|c|c|c|c|c|c|}
\hline 咅 & 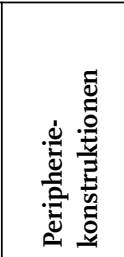 & 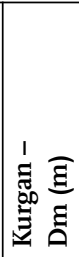 & 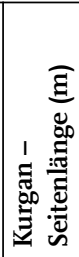 & 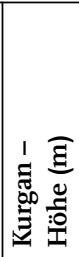 & 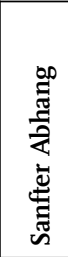 & 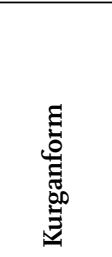 & 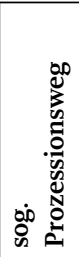 & 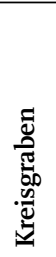 & 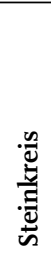 & 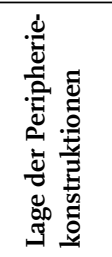 & 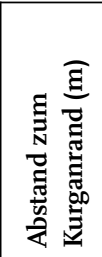 & 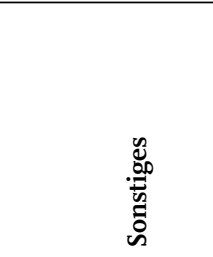 \\
\hline \multirow[t]{2}{*}{$\begin{array}{l}\text { Gf. } \\
\text { Žalauly-2 }\end{array}$} & zum K6I & $\begin{array}{l}3,0 x- \\
0,6\end{array}$ & & 0,2 & & $\begin{array}{l}\text { recht- } \\
\text { eckig }\end{array}$ & & & & SO & $\begin{array}{l}\text { direkt } \\
\text { am } \\
\text { Rand }\end{array}$ & W-O Ausrichtung \\
\hline & zum K6I & \begin{tabular}{|l|}
$2,2 \mathrm{X}-$ \\
0,9
\end{tabular} & & 0,2 & & $\begin{array}{l}\text { recht- } \\
\text { eckig }\end{array}$ & & & & SO & $\begin{array}{l}\text { direkt } \\
\text { am } \\
\text { Rand }\end{array}$ & W-O Ausrichtung \\
\hline $\begin{array}{l}\text { Gf. } \\
\text { Žoan Tobe }\end{array}$ & $\mathrm{KI}_{\mathrm{I}}$ & III & & II & $\mathrm{S}$ & $\begin{array}{l}\text { abge- } \\
\text { rundet }\end{array}$ & $\mathrm{x}$ & & & $\begin{array}{l}\text { um den } \\
\text { Kurgan }\end{array}$ & überall & $\begin{array}{l}28 \text { Kleinkurgane } \\
\text { (Dm: 3,6-8,5m) }\end{array}$ \\
\hline \multirow[t]{4}{*}{$\begin{array}{l}\text { Gf. } \\
\text { Ž́lysaj-1 }\end{array}$} & K6 & & 28 & 3 & S & $\begin{array}{l}\text { vier- } \\
\text { eckig }\end{array}$ & $\mathrm{x}$ & & & $\mathrm{N}$ & 3 & $\begin{array}{l}2 \text { frühtürkische } \\
\text { Kurgane }\end{array}$ \\
\hline & $\mathrm{K}_{7}$ & 39 & & 3 & S & $\begin{array}{l}\text { abge- } \\
\text { rundet }\end{array}$ & $\mathrm{x}$ & & & $\mathrm{N}$ & I & $\begin{array}{l}2 \text { frühtürkische } \\
\text { Kurgane }\end{array}$ \\
\hline & K9 & $2 \mathrm{I}$ & & 2 & S & $\begin{array}{l}\text { abge- } \\
\text { rundet }\end{array}$ & $\mathrm{x}$ & & & $\mathrm{N}$ & 2 & $\begin{array}{l}\text { frühtürkischer } \\
\text { Kurgan }\end{array}$ \\
\hline & $\begin{array}{l}\text { Kio } \\
\text { (Klein- } \\
\text { kurgan) }\end{array}$ & & 5 & 0,2 & & $\begin{array}{l}\text { quadra- } \\
\text { tisch }\end{array}$ & & & & & & \\
\hline
\end{tabular}

ren Peripherie Befunde aufweisen, treten in allen Verbreitungszonen früheisenzeitlicher Gräberfelder mit Großkurganen der sakischen Elite auf. Fast alle diese Kurgane haben eine abgerundete, plattformähnliche Form mit abgeflachter Kuppe. Ausnahmen stellen ein Kurgan 5 km südwestlich vom Dorf Žalauly303 und Kurgan 6 des Gräberfeldes Žylysaj-I304 dar, da sie eine viereckige, pyramidenähnliche Form mit abgeflachter Kuppe aufweisen. Nur zwei Kurgane (ein Kurgan 5 km südwestlich vom Dorf Žalauly305 und Gf. Boroldaj, $\mathrm{KI}_{\mathrm{I}}{ }^{306}$ ) hatten an allen Seiten gleichmäßige Abhänge. Die restlichen hatten drei steile und einen sanften Abhang. Bei über der Hälfte der Kurgane (I5 Grabhügeln; 68\%) befindet sich der sanfte Abhang exakt im Süden. Bei drei Kurganen 307 (I4\%) ist dieser Abhang nach Südosten ausgerichtet. Jeweils ein Kurgan mit Befunden in der Peripherie hat den sanften Abhang im Osten308 bzw. Südwesten309.

Am Rande von I4 Kurganen wurden weitere Konstruktionen wie sog. Prozessionswege, Kreisgräben oder Steinkreise festgestellt.

Bei diesen Befunden in der Peripherie der Kurgane handelt es sich um kleine, flache, abgerundete bzw. ovale Steinanhäufungen, die möglicherweise Kleinkurgane darstellen. Zudem treten noch rechteckigen Vertiefungen oder steinkistenähnliche Konstruktionen auf, deren Ansprache bislang unklar bleibt. Ferner kamen noch flache, quadratische Kurgane zum Vorschein, die mit der frühtürkischen Periode zu verbinden sind (vgl. Bernštam I949b, 365-366; vgl. Vasjutin 2009, 82-90, Abb. 4-5, 7). Diese Peripheriekonstruktionen wurden entweder zeitgleich mit dem Großkurgan errichtet oder aber später.

303 Siehe Katalog, ein Kurgan 5 km südwestlich vom Dorf Žalauly.

304 Siehe Katalog, Gf. Žylysaj-I, Kurgancharakteristik.

305 Siehe Katalog, ein Kurgan 5 km südwestlich vom Dorf Žalauly.

306 Siehe Katalog, Gf. Boroldaj, Kurgancharakteristik.
307 Gf. Issyk, K2I-22 und K30 (siehe Katalog, Gf. Issyk, Kurgancharakteristik).

308 Gf. Issyk, K9 (siehe Katalog, Gf. Issyk, Kurgancharakteristik).

309 Gf. Asy Saga, K4 (siehe Katalog, Gf. Asy Saga, Kurgancharakteristik). 

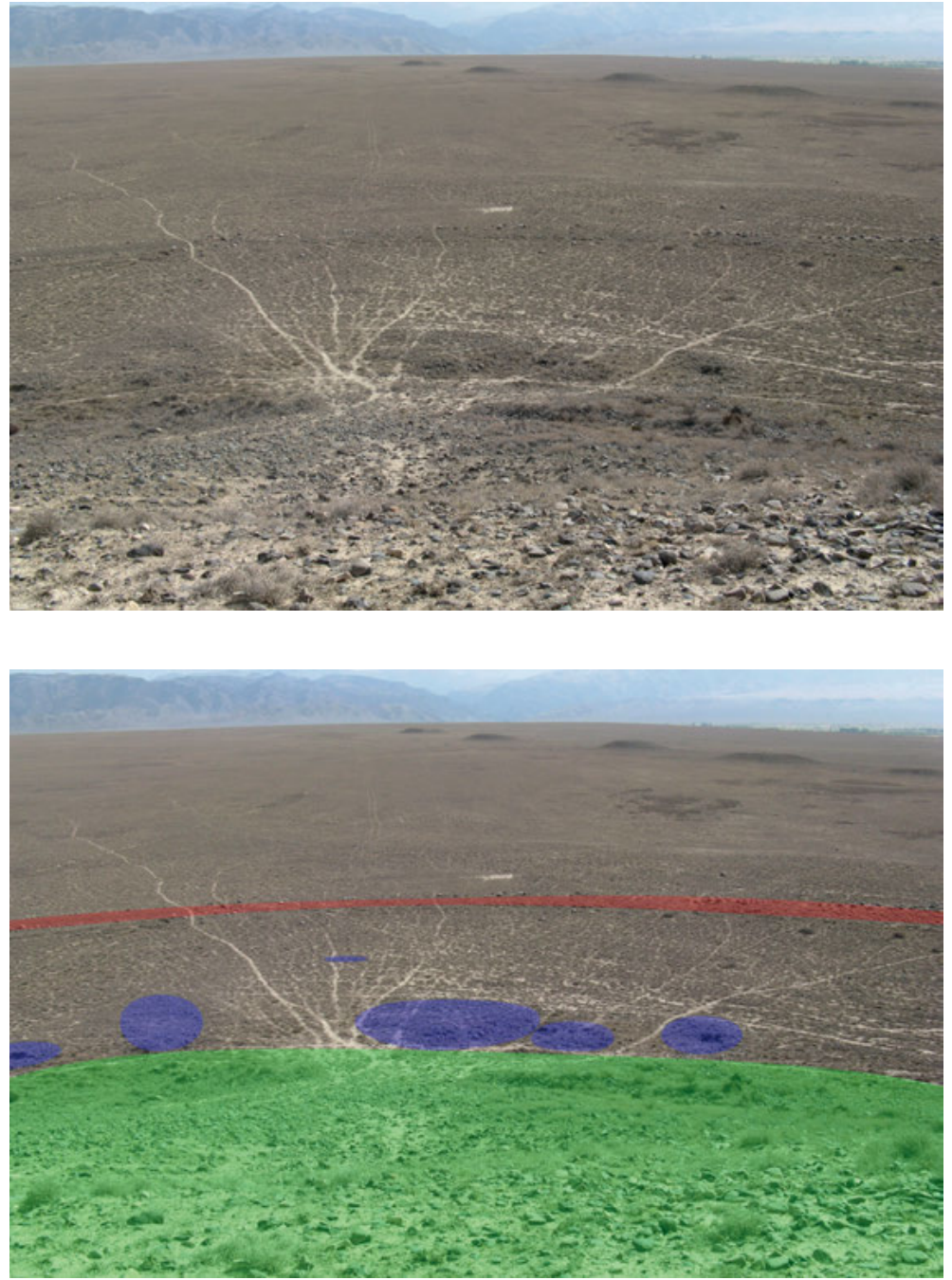

Abb. 54 | Gräberfeld Asy Saga. Südliche Peripherie des Kurgans I. Blick vom Kurgan I Richtung Süden

\author{
Abb. 55 | Gräberfeld Asy Saga. Südliche \\ Peripherie des Kurgans I (grün - Abhang \\ des Kurgans und Kurganrand; rot - sog. \\ Prozessionsweg; blau - abgerundete, \\ kleine Steinkonstruktionen/ Klein- \\ kurgane ?)
}

Die kleinen, flachen, abgerundeten Steinanhäufungen, die man als Kleinkurgane interpretieren kann, treten im Zusammenhang mit Großkurganen in unterschiedlicher Häufigkeit auf. In sechs Fällen fand sich nur ein einziger solcher Befund neben einem Großkurgan, in zwei Fällen zwei Peripheriekonstruktionen; drei, vier, acht, 2I, 28 und 4I Befunde traten jeweils nur einmalig neben einem Kurgan auf. Insgesamt befinden sich in der Peripherie der aufgenommenen Kurgane II5 Kleinkurgane (84\% aller Peripheriekonstruktionen), deren Höhen zwischen o,I $\mathrm{m}$ bis I,2 $\mathrm{m}$ schwanken und die Durchmesser von $3 \mathrm{~m}$ bis $20 \mathrm{~m}$ aufweisen. Zehn solcher Kleinkurgane (IO\% aller Peripheriekonstruktionen) lagen südlich der Großkurgane. Fünf weitere Anlagen (4\%) befanden sich nordwestlich der Großkurgane. Weitere vier Kleinkurgane (3\%) wurden östlich der Großkurgane errichtet. Zwei Anlagen kamen nordöstlich der Großkurgane zum Vorschein. Jeweils ein Kleinkurgan befand sich südöstlich bzw. südwestlich vom Großkurgan. Weitere 93 Kleinkurgane $(80 \%)$ wurden in der Peripherie von insgesamt vier Großkurganen festgestellt. Sie wurden um die Kurgane herum errichtet. Es fällt auf, dass sich an der südlichen und östlichen Seite der Großkurgane wesentlich mehr Kleinkurgane befanden, als an den übrigen Seiten. Süd- und Ostseite scheinen also die bevorzugte Lage für die Errichtung von Peripherieanlagen der Großkurgane gewesen zu sein. Die Kleinkurgane schlossen direkt am Rand eines Großkur- 


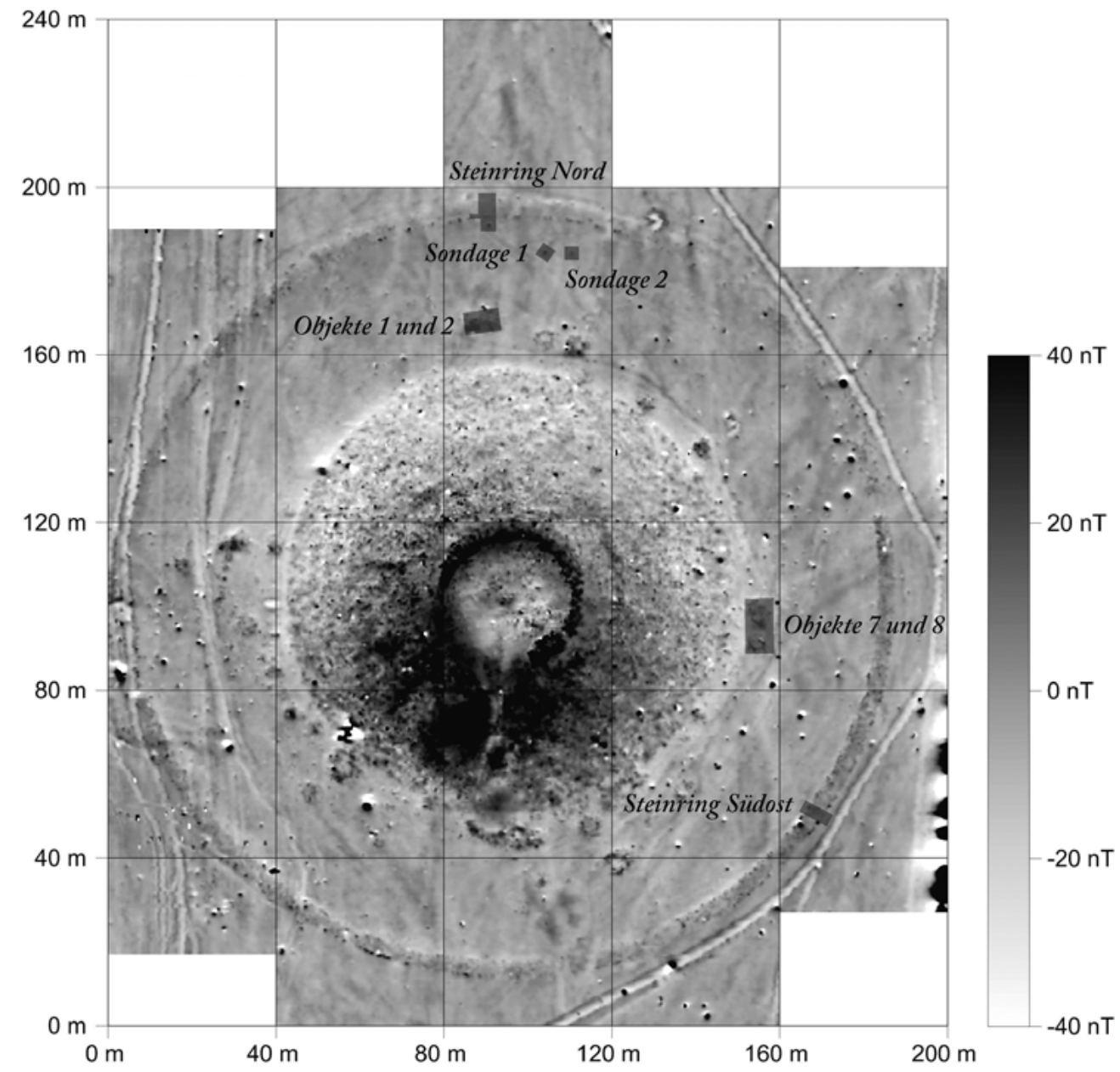

Abb. 56 | Gf. Žoan Tobe. Kurgan I. Verteilung der untersuchten Objekten der Peripherie. Ergebniss der Magnetomessung (Caesium Smartmag SM4G special Magnetometer in duo-sensor Konfiguration, Empfindlichkeit +/- Io Picotesla). Dynamik +/- 2I nT in 256 Graustufen von Schwarz bis Weiß; reduziert auf den Mittelwert, Messgitter $40 \times 40$ Meter; Abtastrate I2,5 $\times 50 \mathrm{~cm}$; Das Erdmagnetische Totalfeld im August 2008 ca. $55520+/-20$ Nanotesla. Norden ist oben (Bildnachweis und Datenverarbeitung: J. Faßbinder BLfD München)

gans an. Es fanden sich aber auch Kleinkurgane in einem Abstand von $0,3 \mathrm{~m}$ bis zu $37 \mathrm{~m}$ vom Rand eines großen Grabhügels. Im Durchschnitt lagen I4 $\mathrm{m}$ zwischen Kleinkurgan und Rand des Großkurgans. Im Umfeld von Kurgan 3 des Gräberfeldes Turgen ${ }^{\text {10 }}$ und Kurgan I des Gräberfeldes Žoan Tobe3rI wurden einige wenige Kleinkurgane untersucht. Es handelt sich um die Gräber 2 und 5, die am östlichen Rand des Kurgans 3 des Gräberfeldes Turgen errichtet worden waren (Dublickij I939a, 5-8) und um die Objekte $^{32}$ I, 2 und 7, die nördlich und östlich vom Rand des Kurgans I der Nekropole Žoan Tobe lagen313

3IO Siehe Katalog, Gf. Turgen, Kurgancharakteristik.

3II Siehe Katalog, Gf. Žoan Tobe, Kurgancharakteristik.

3I2 In diesem Falle wurde der Terminus aus der Originaldokumentation übernommen.
3I3 Für Informationen zur Untersuchung der Objekte I, 2 und 7, die im Sommer 2008 durch die Kasachisch-Deutsche Archäologische Expedition vorgenommen wurden, möchte ich mich bei H. Parzinger, Z. Samašev, A. Nagler, M. Nawroth und M.-R. Boroffka herzlich bedanken. 

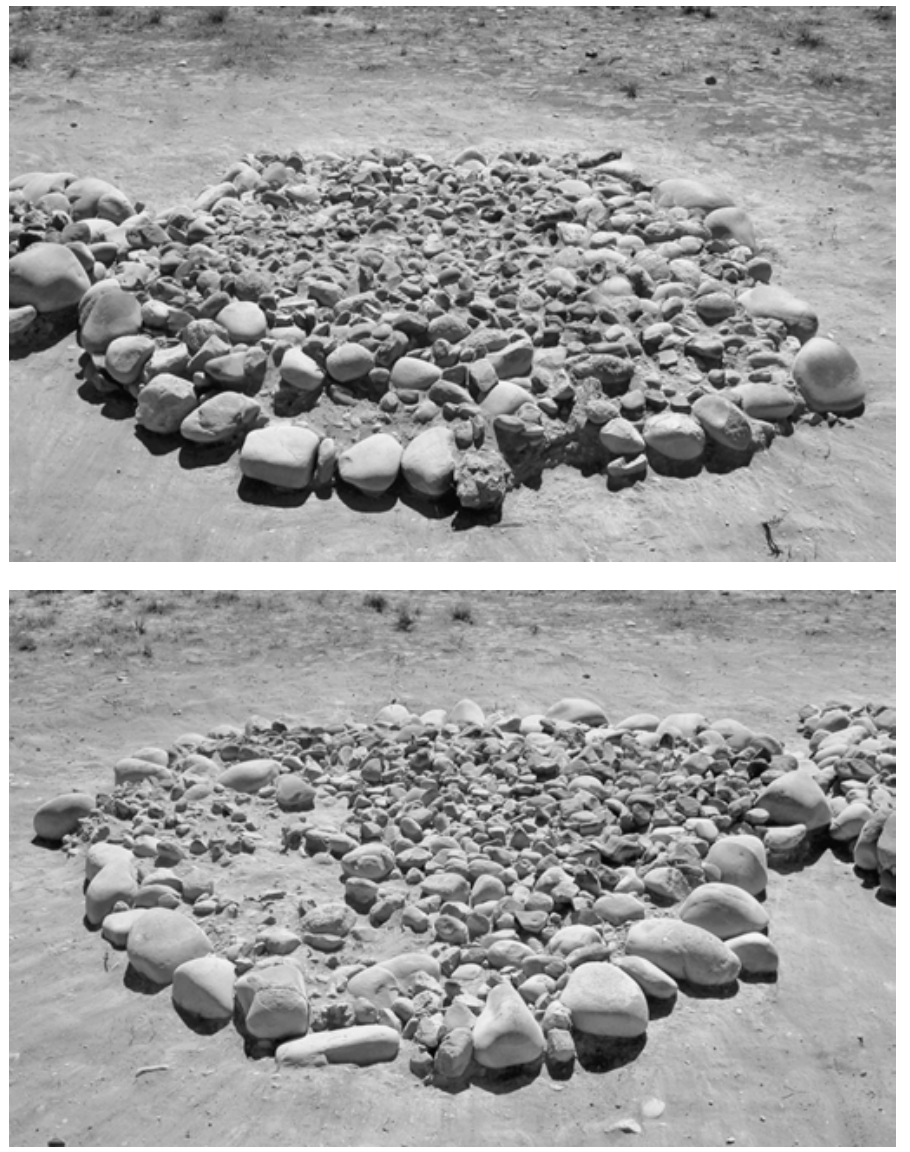

Abb. 57 | Gf. Žoan Tobe. Kurgan I. Objekt I. Planum I. Blick von N (Foto M. Nawroth)

Abb. 58 | Gf. Žoan Tobe. Kurgan I. Objekt 2. Planum I. Blick von N (Foto M. Nawroth)

(Abb. 56). Diese Konstruktionen erwiesen sich als ovale bzw. abgerundete Steinbauten mit einer Höhe von o,I bis 0,2 $\mathrm{m}$ und einem Durchmesser von 4 bis $7 \mathrm{~m}$. Die äußere Grenze wurde durch größere Steine markiert.

In der Mitte dieser Objekte befand sich je eine Grabgrube von länglicher, ovaler oder rechteckiger Form mit abgerundeten Ecken und Ausmaßen von 3,O-I,O × 2,5-O,5 × 3,O-O,35 m¹4. Die Steinbauten waren entweder nord-südlich (drei Gräber) oder nordwest-südost (zwei Gräber) ausgerichtet. Auf dem Grabboden befand sich jeweils ein Skelett eines erwachsenen Individuums in Rückenstreckerposition mit dem Kopf nach Norden (drei Individuen), Westen (ein Individuum) oder Nordwesten (ein Individuum). Beigaben fehlten in allen fünf Gräbern völlig. Dabei könnte es sich hier sowohl um die zeitgleich mit dem Großkurgan errichteten als auch um die später gebauten Befunde handeln.

Um sich mit den Einzelheiten der Kleinkurgane intensiver zu beschäftigen, empfiehlt es sich, die Untersuchungen zum Kurgan I auf dem Gräberfeld Žoan Tobe der Kasachisch-Deutschen Archäologischen Expedition in Betracht zu ziehen. Da es vor der Untersuchung nicht klar gewesen war, ob es sich um einen Kleinkurgan oder um eine andere Art des Befundes handelt, wurde entschieden, die untersuchten Konstruktionen als Objekte zu bezeichnen.

Objekt I befand sich 8,4 m nördlich vom Kurganrand und grenzte westlich an das Objekt 2 (Abb. 56-59). Objekt I stellte eine ovalförmige Konstruktion dar, die ein Ausmaß von 4,2 × 3,6 m hatte 

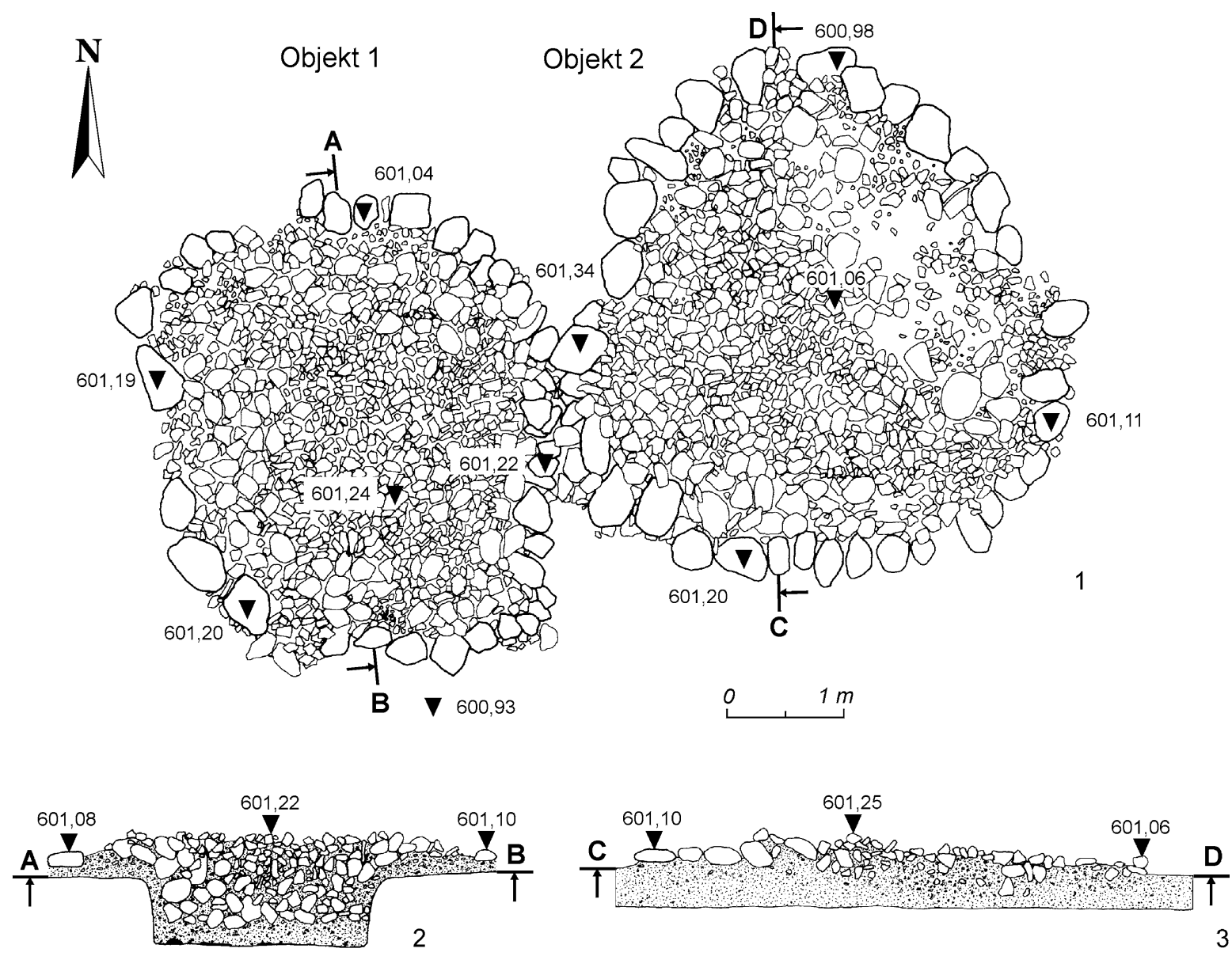

Abb. 59 | Gf. Žoan Tobe. Kurgan I. Objekte I und 2. Planum I und Profile (Zeichnung M.-R. Boroffka)

und eine Höhe von 12 cm erreichte. Es bestand aus einem ovalen Kranz aus größeren Steinen, die auf groben Kies eingebettet wurden. Der Steinkranz wurde von ein bis zwei Schichten der mittelgroßen Steine gefüllt und zum Teil überdeckt. Die Zwischenräume der Steine wurden, um sie zu stabilisieren, mit dem Kies aufgefüllt. Die Steinkonstruktion überlagerte eine in der Mitte angelegte langovale Grabgrube, die 2,2 × I,2 m groß und o,6 m tief war (Abb. 6o). Die Grabgrube wurde nordnordwest-südsüdöstlich ausgerichtet und mit den großen sowie mittelgroßen Steinen und Kies verfüllt. Auf dem Grabboden lag ein Skelett mit am Körper angelegten Armen in Rückenstreckerlage, den Kopf nach Nordnordwesten ausgerichtet (Abb. 6I-62). Den Erhaltungszustand der Knochen kann man als eher schlecht bezeichnen. Die Bestattung der Grabgrube im Objekt I war ohne Beigaben.

Objekt 2 grenzte östlich an das Objekt I und befand sich 8,2 m nördlich vom Rand des Großkurgans I (Abb. 56, 58-59). Objekt 2 stellte eine eiförmige Steinpackung dar (4,6 m × 4,4 m; Höhe 0,I5 m). Wie Objekt I bestand Objekt 2 aus einem Kranz großer Steine, die auf groben Kies gebettet worden sind. Der Innenraum des Steinkranzes wurde mit mittelgroßen sowie kleinen Steinen und dem Kies verfüllt, die ein bis zwei Schichten bildeten.

In der Mitte des Objekts 2 im gewachsenen Boden wurde eine rechteckige Grabgrube mit abgerundeten Ecken ausgehoben. Die nordwest-südöstlich ausgerichtete Grabgrube maß 2,3 × I,I5 m und war o,6 m tief. Die Grabgrube wurde mit mittelgroßen Steinen und mit Kies verfüllt (Abb. 63). 

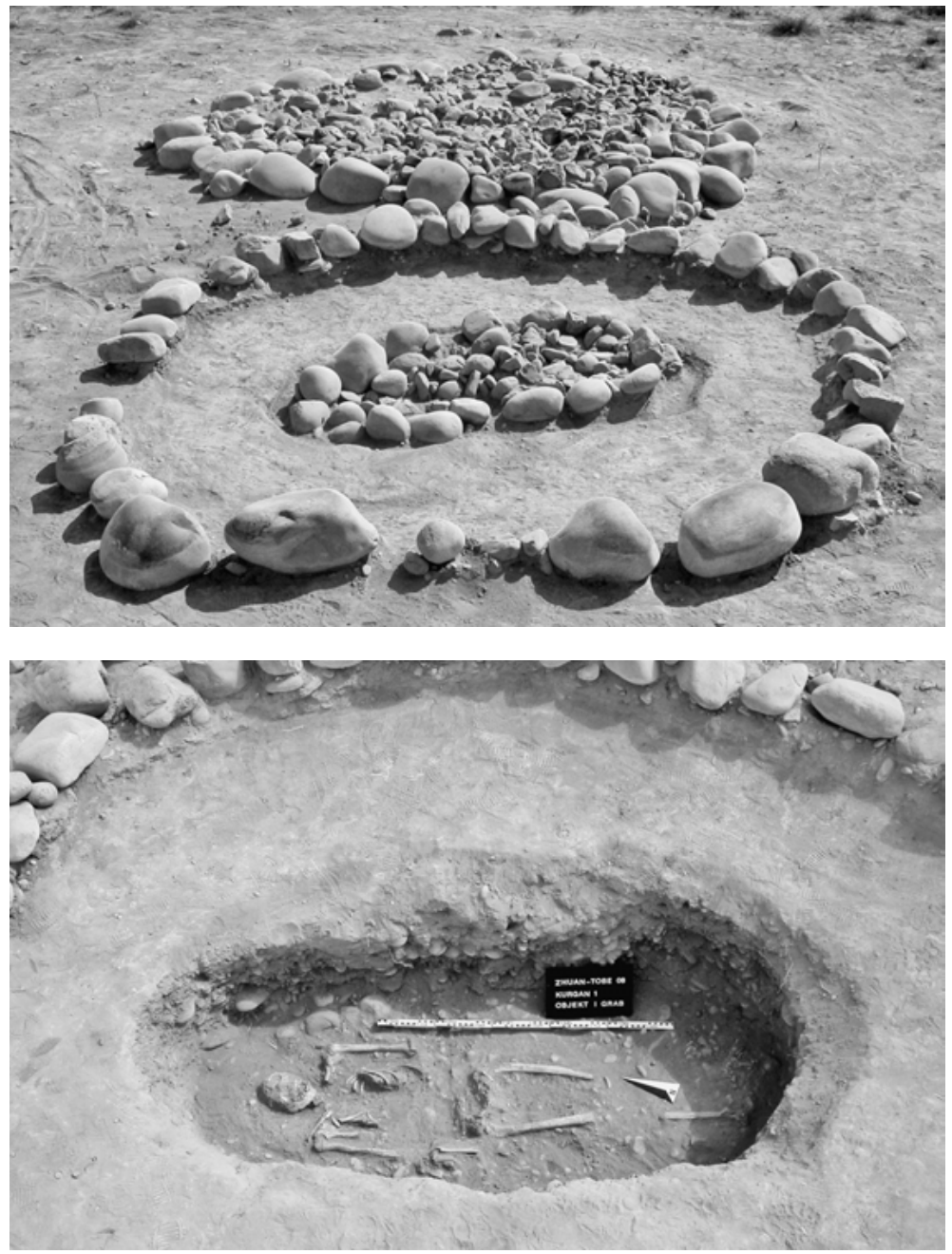

Abb. 6o | Gf. Žoan Tobe. Kurgan I. Objekt I. Planum 2. Blick von W (Foto M. Nawroth)
Abb. 61 | Gf. Žoan Tobe. Kurgan I. Objekt I. Grab (Foto M. Nawroth)

Auf dem Boden der Grube lag ein Skelett in Rückenstreckerlage mit dem Kopf nach Nordwesten (Abb. 62, Abb. 64). Der Kopf des bestatteten Individuums wurde leicht auf die linke Seite gekippt, die Arme wurden am Körper angelegt. Die Grabgrube wies keine Beigaben auf. Die Bestattung im Objekt 2 des Kurgans I auf der Nekropole Žoan Tobe wurde absolutchronologisch in das IO.-II. Jh.315 datiert.

Eine chronologische Abfolge der Errichtung beider Objekte lässt sich aufgrund der Beobachtungen der horizontalen Stratigraphie oder der Besonderheiten des Bestattungsrituals nicht rekonstruieren.

Objekt 7 befand sich direkt am östlichen Rand des Großkurgans I (Abb. 56). Im Laufe der Freilegung dieses Objekts wurde auch die südlich von ihm gelegene, benachbarte Steinkonstruktion, Objekt 8, aufgenommen (Abb. 65-66). Jedoch wurde eine komplette Untersuchung des Objekts 8 nicht durchgeführt.

Objekt 7 stellte eine Steinkonstruktion mit einer unregelmäßigen, eher abgerundeten Form (7,I $\times$ 7,0 m; Höhe o,2 m) dar. Deutlich größere Steine, von denen mehrere fehlten, bildeten einen ovalen Steinkranz $(6,5 \times 6,0 \mathrm{~m})$. Die großen Steine der Kranzmarkierung lagen direkt auf dem alten Laufho- 


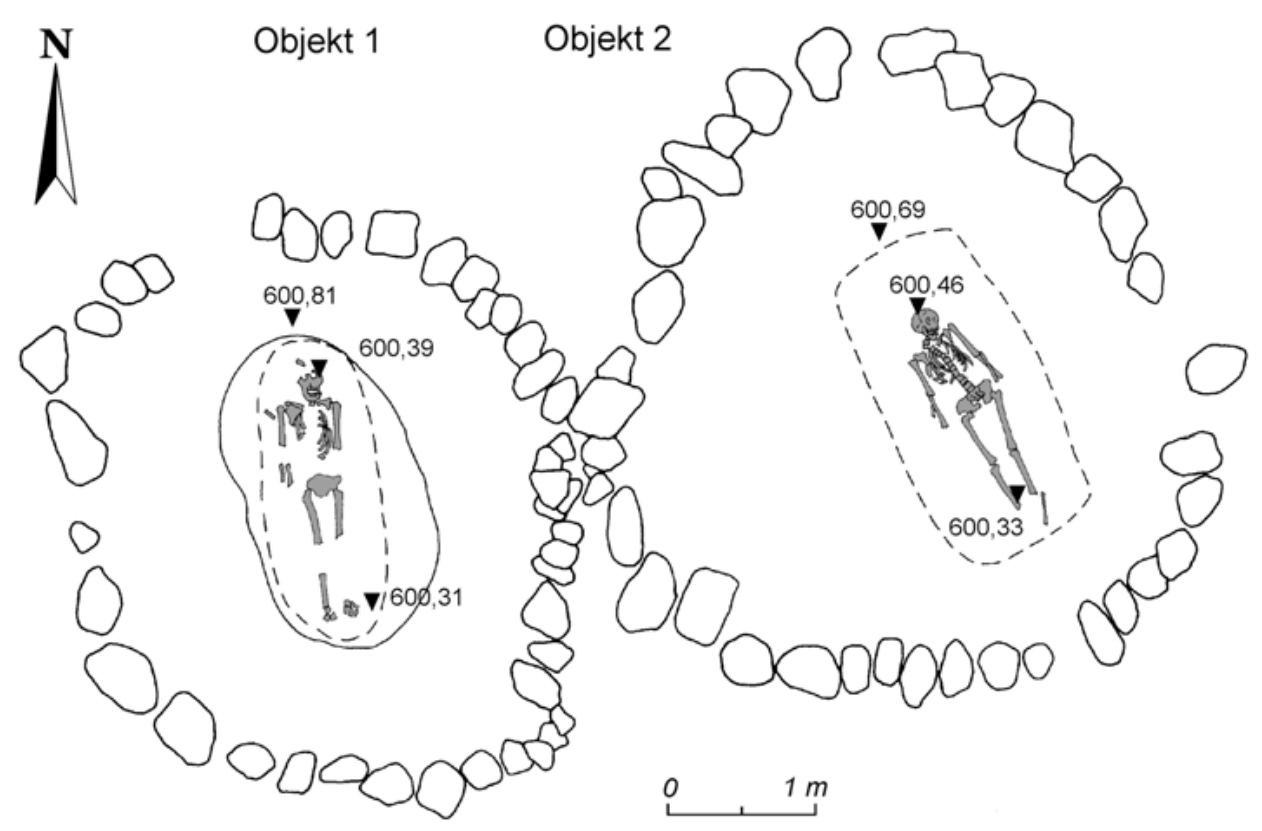

Abb. 62 | Gf. Žoan Tobe. Kurgan I. Objekte I und 2. Planum 2 mit eingetragenen Gräbern (Zeichnung M.-R. Boroffka)

rizont. Dieser Kranz wurde mit vier bis fünf Schichten mittelgroßer sowie kleiner Steine und dem Kies verfüllt, so dass an einigen Stellen diese Verfüllung den Steinkranz überlagerte.

Beim Freilegen des großen äußeren Kranzes kam im Innenen ein weiterer, kleinerer Steinkranz zum Vorschein, der teilweise nur aus wenigen kleineren Steinen bestand (Abb. 67). Dieser innere Kranz wies eine ovale Form $(5,3 \times 4,5 \mathrm{~m})$ auf. Sein nordwestliches Segment fehlte komplett. Im Zentrum des Objektes 7 wurde eine schmale, langovale Grabgrube festgestellt. Die nordnordost-südsüdwestlich ausgerichtete Grube maß 2,00 × 0,55 $\mathrm{m}$ und war 0,4 $\mathrm{m}$ tief. Die Grabgrube wurde mit Kies und mittelgroßen bis kleinen Steinen verfüllt, zudem kam noch ein Stück eines linken Schulterblattes vom Pferd ${ }^{15}$ zum Vorschein. Auf dem Boden der Grabgrube lagen einige wenige Fragmente eines schlecht erhaltenen Skelettes (Abb. 68-69). Im mittleren Bereich der Grube wurden einige Schädelfragmente geborgen, die sich in sekundärer Lage befanden. Entlang der nordöstlichen Grubenwand lag ein Oberarmknochen. In der Südhälfte der Grabgrube kamen die gestreckten Beinknochen zum Vorschein, die noch in situ waren. Die Knochen der unteren Extremitäten lassen vermuten, dass das bestattete Individuum sich in Rückenstreckerlage mit dem Kopf nach Norden befand. Die Anzeichen einer Beraubung oder einer intentionellen Störung fehlten. Das Skelett wurde höchstwahrscheinlich von Tieren gestört. Ebenso wie die Bestattungen von Objekt I und 2 enthielt die Bestattung im Grab von Objekt 7 keinerlei Beigaben.

Objekt 8 wurde in unmittelbarer Nähe zu Objekt 7 gebaut (Abb. 66). Die Steinpakung des Objekts hatte eine unregelmäßige, eher abgerundete Form $(6,0 \times 5,2 \mathrm{~m})$. Heraus ragten deutlich größere Steine, die einen Steinkranz zu bilden scheinen, wie in oben beschriebener Situation.

3I6 Archäozoologische Bestimmung von Prof. Dr. rer. nat. Norbert Benecke, Deutsches Archäologisches Institut,
Naturwissenschaftliches Referat an der Zentrale, Archäozoologie. 

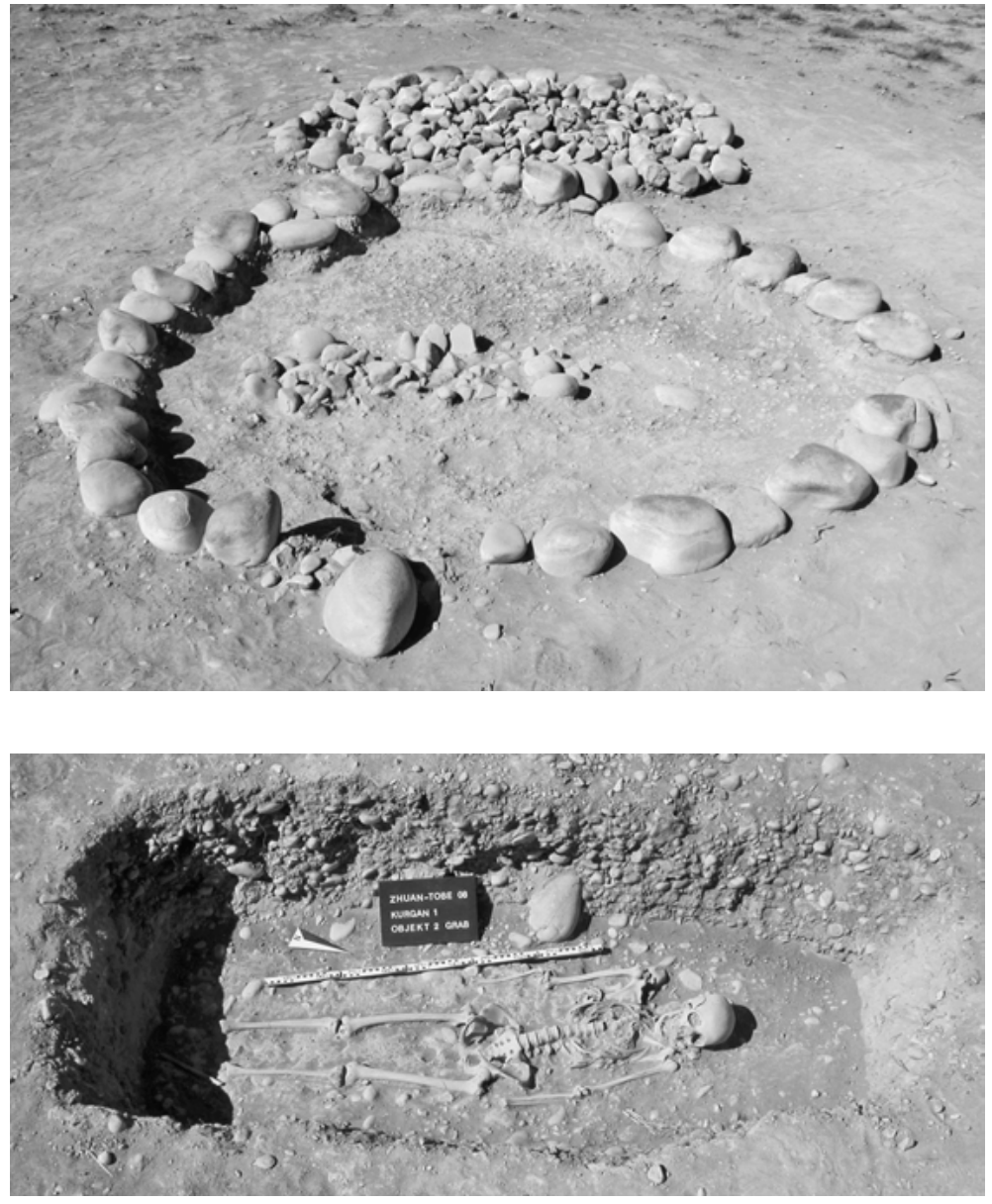

Abb. $6_{3} \mid$ Gf. Žoan Tobe. Kurgan I. Objekt 2. Planum 2. Blick von O (Foto M. Nawroth)

Abb. 64 | Gf. Žoan Tobe. Kurgan I. Objekt 2. Grab (Foto M. Nawroth)

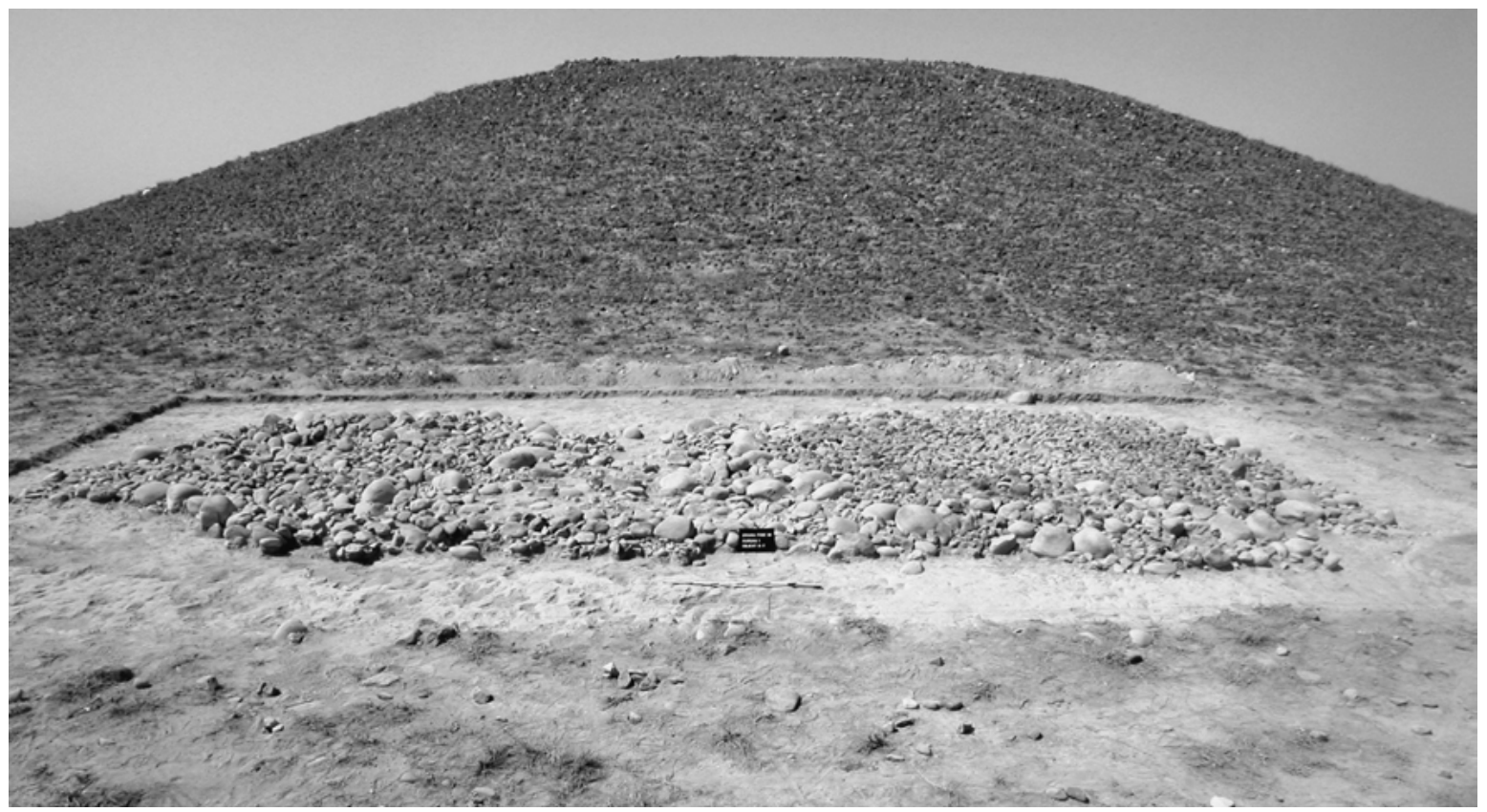

Abb. $65 \mid$ Gf. Žoan Tobe. Kurgan I. Objekte 7 und 8. Planum I. Blick von O (Foto M. Nawroth) 


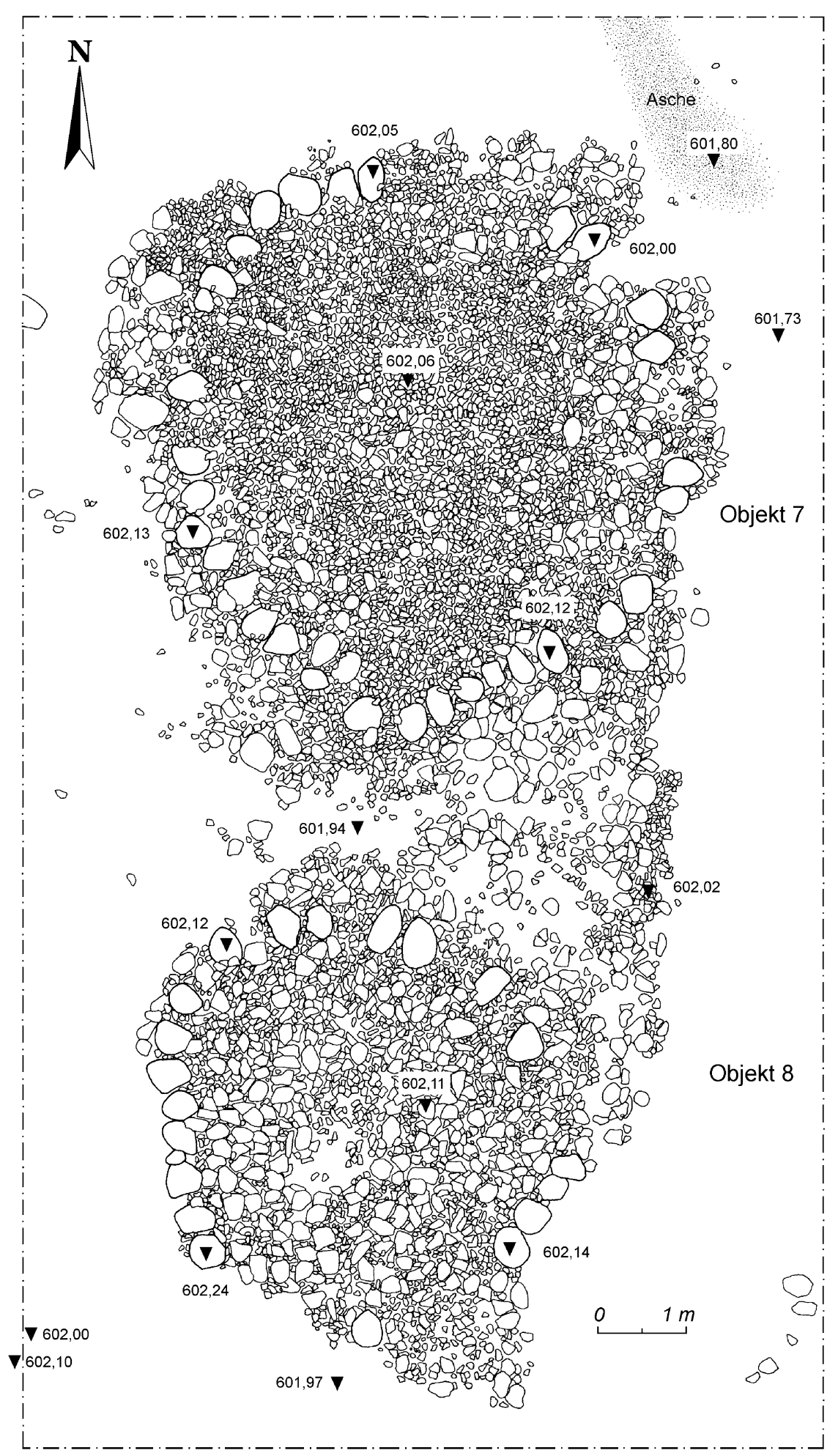

Abb. 66 | Gf. Žoan Tobe. Kurgan I. Objekte 7 und 8. Planum I (Zeichnung M.-R. Boroffka) 


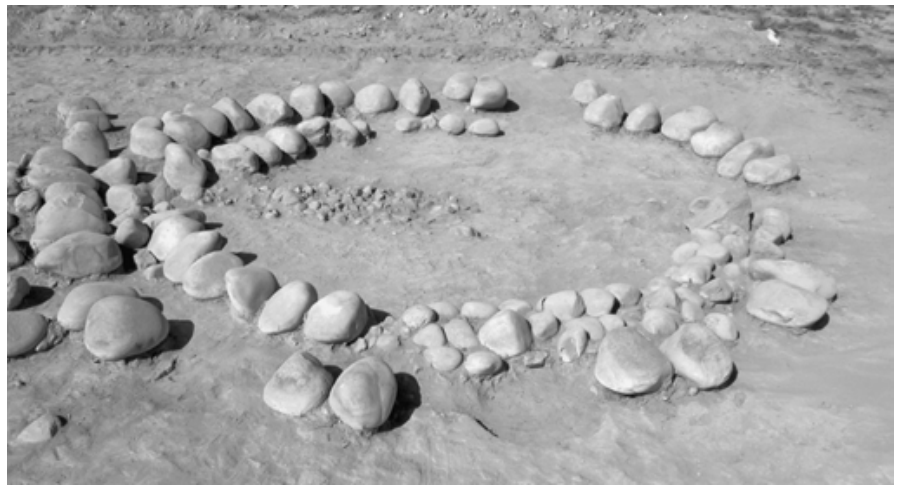

Abb. $67 \mid$ Gf. Žoan Tobe. Kurgan I. Objekt 7. Planum 2. Blick von O (Foto M. Nawroth)
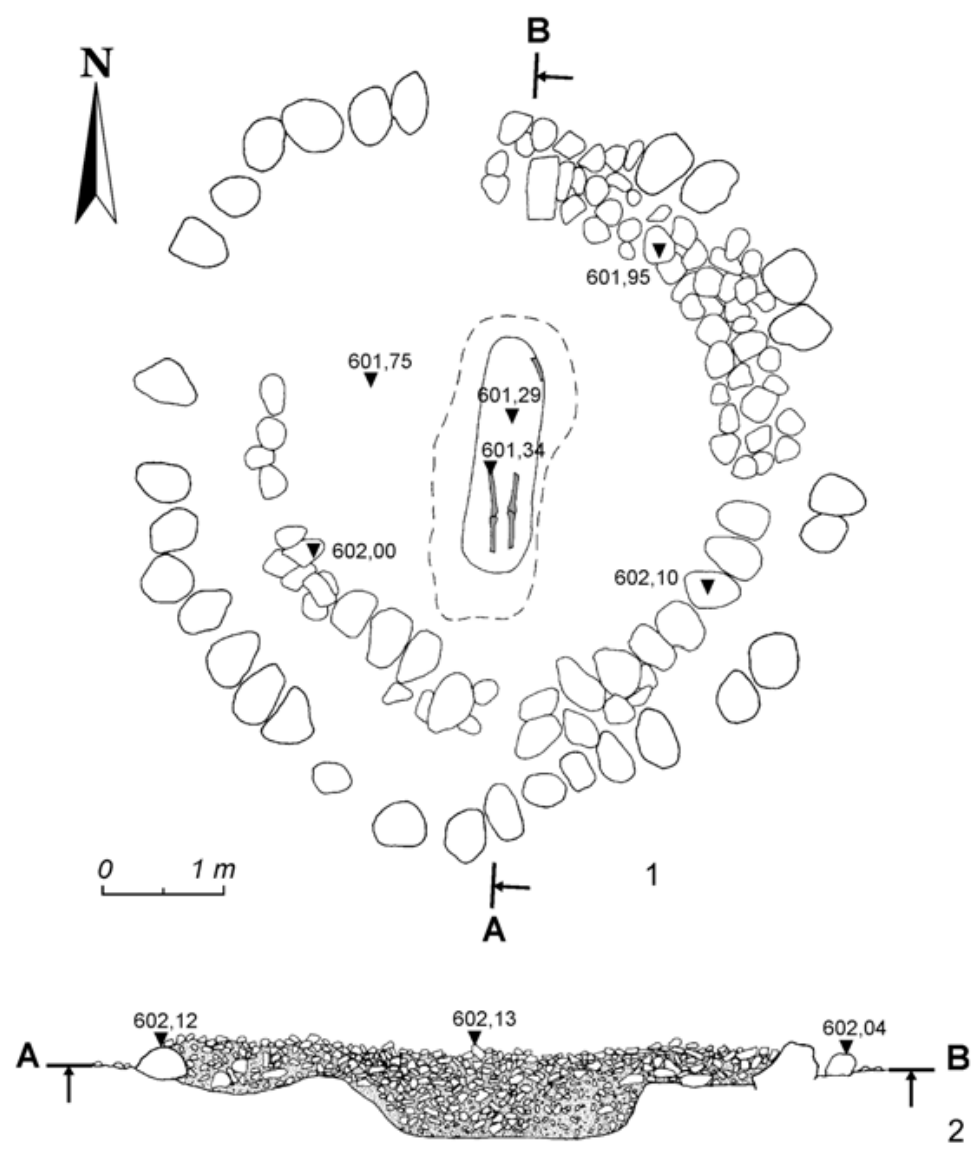

Abb. 69 | Gf. Žoan Tobe. Kurgan I. Objekt 7. Planum 2 mit eingetragener Grabgrube und der Profil des Objektes (Zeichnung M.-R. Boroffka)

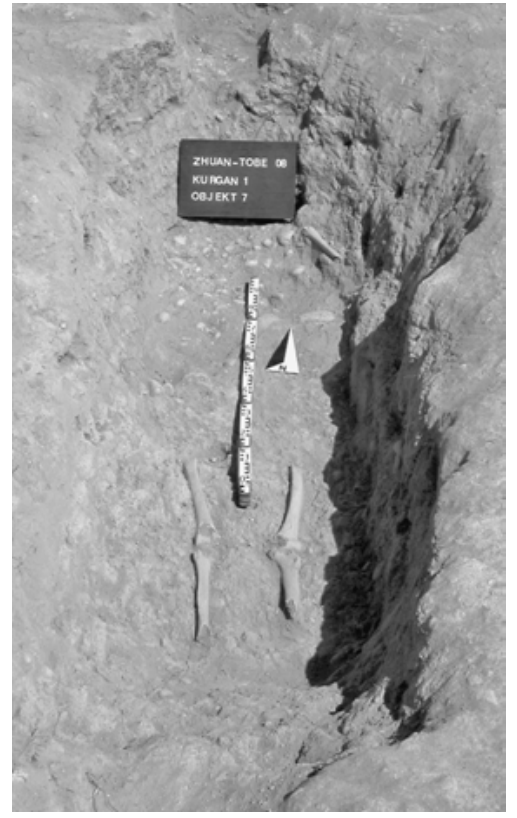

Abb. 68 | Gf. Žoan Tobe. Kurgan I. Objekt 7. Grab (Foto M. Nawroth) 
In der Peripherie der Kurgane II vom Gräberfeld Kyzylšaryk317 und 6I der Nekropole Žalauly-2318 kamen steinkistenähnliche Konstruktionen zum Vorschein ( $6 \%$ aller Peripheriekonstruktionen). Insgesamt handelt es sich um acht rechteckige Konstruktionen, die aus 50-30 ×30-20 × 20-10 cm großen, senkrecht aufgestellten Steinen bestanden. Der innere Bereich war frei von Steinen. Diese steinkistenähnlichen Konstruktionen maßen 4,O-2,9 × 2,I-0,5 $\mathrm{m}$ und waren in 62\% der Fälle (fünf Konstruktionen) west-östlich ausgerichtet. Drei Steinkisten wiesen eine Nord-Süd Achse auf und befanden sich jeweils in drei Fällen westlich bzw. südöstlich vom Großkurgan, zwei weitere wurden östlich davon errichtet. Sie befanden sich entweder direkt am Rand des Großkurgans oder in einer Entfernung von 9 bis $15 \mathrm{~m}$.

Auf dem Gräberfeld Žylysaj-1¹9, nördlich der Kurgane 6, 7 und 9, in einem Abstand von I bis 3 m, traf man $5 \times 5$ m oder $2 \times 2$ m große, flache, quadratische Kurgane an (fünf Stück; $4 \%$ aller Peripheriekonstruktionen). Sie sind als frühtürkisch zu klassifizieren.

Nördlich vom Kurgan 4 des Gräberfeldes Aktasty-3230 wurden fünf rechteckige Vertiefungen $(2,0-2,5 \times$ I, O $\mathrm{m}$ ) festgestellt ( $4 \%$ aller Peripheriekonstruktionen). Diese befanden sich $2 \mathrm{~m}$ vom Rand des Kurgans entfernt, wobei jeweils die schmalen Seiten dem Kurgan zugewandt waren. Die Bedeutung dieser Befunde sowie ihre chronologische Stellung sind ohne Ausgrabung nicht zu klären.

Drei kreisförmige Wälle (Abb. 70) lagen II m nördlich und nordwestlich vom Kurgan 9 des Gräberfeldes Issyk ${ }^{22 I}$ entfernt. Der Durchmesser der Wälle betrug ı-I8 m. Nach der Form zu urteilen, liegt eine Datierung in kasachisch-ethnographische Zeit nahe.

Abb. 70 | Kreisförmige Wälle in der Peripherie des Kurgans 9 des Gräberfeldes Issyk. Blickvon SO

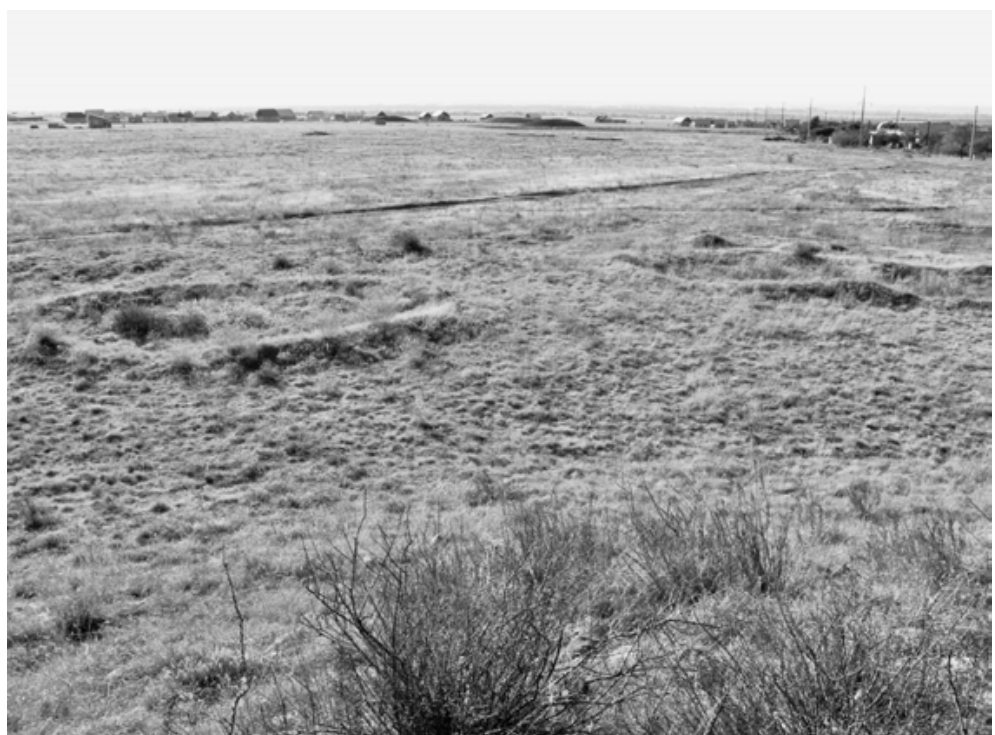

3I7 Siehe Katalog, Gf. Kyzylšaryk, Kurgancharakteristik.

3I8 Siehe Katalog, Gf. Žalauly-2, Kurgancharakteristik.

3I9 Siehe Katalog, Gf. Žylysaj-I, Kurgancharakteristik.
320 Siehe Katalog, Gf. Aktasty-3, Kurgancharakteristik.

32I Siehe Katalog, Gf. Issyk, Kurgancharakteristik. 


\subsubsection{Sog. Prozessionswege}

Auf neun Gräberfeldern wurden bei insgesamt i9 Kurganen322 (Tabelle I7; Karte i3) den Hügel umlaufende sog. Prozessionswege festgestellt (Abb. 54-55 und Abb. 7I). Dabei handelt es sich um doppelte Steinkreise, die sich in einem Abstand von $0,5-5,0 \mathrm{~m}$ zueinander befanden und im Innenraum mit mittelgroßen bis kleinen Flusskieselsteinen sowie mit Lehm vermischtem Schotter bzw. gestampften Steinschutt verfüllt waren.

Zum ersten Mal wurden solche doppelten Steinkreise im Jahre 2008 durch die Kasachisch-Deutsche Archäologische Expedition auf dem Gräberfeld Žoan Tobe um den Kurgan I herum323 archäologisch untersucht (Abb. 72). Die Konstruktion bekam eine laufende Nummerierung „Objekt 3I“ und wurde an zwei Stellen angeschnitten, nämlich im nördlichen (Objekt 3I-Nord) und im östlichen (Objekt 3I-Ost) Bereich. Die Befundsituation in beiden Schnitten war identisch324. Der gewachsene Boden war auf einer Breite von 4,5-5, O m mit mittelgroßen Kieselsteinen gepflastert. Die Seiten waren durch große, längliche Grenzsteine markiert, so dass eine Art „Wegebett“ entstand. Das wurde mit einer lehmigen, mit Steinschutt vermischten Schicht verfüllt. Die oberste bestand aus gestampftem Schotter (Samašev u.a. 2009, 35I, Gass 20I2, 47I). Der mittlere Bereich war im Vergleich zum Rand um ein paar Zentimeter erhöht. Dadurch konnte ein besserer Regenwasserablauf gewährleistet werden (Gass 2orıb, 213). Die gesamte Konstruktion erreichte eine Höhe von 0,20-0,25 m. Die Ergebnisse der Untersuchung zeigen, dass es sich hier nicht nur um doppelte Steinkreise handelt, sondern um eine aufwendig angelegte straßenpflasterähnliche Konstruktion bzw. „Prozessionsweg“. Diese Bezeichnung des Befundes325 wurde zuerst 2009 in der russischsprachigen Literatur eingeführt (Samašev u.a. 2009, 35I) und im Nachhinein übernommen. Es soll jedoch angemerkt werden, dass es sich hier ausschließlich auf den metaphorischen Charakter des Begriffs bezieht, da es momentan keinerlei Anhaltspunkte dafür gibt, dass solche Konstruktionen zu Prozessionen oder ähnlichen rituellen Handlungen genutzt wurden. Es ist vorstellbar, dass der sog. Prozessionsweg einen rituellen Raum um den Kurgan herum markierte und ähnlich wie bei Kreisgräben oder Wällen eine architektonisch etwas aufwendiger gestaltete Abgrenzung des Totenbereiches von der weiteren Umgebung symbolisierte.

Nichtsdestotrotz sind solche Befunde die ältesten Zeugnisse der Straßenbautechnik in Mittelasien (Samašev u.a. 2009, 35I; Nagler 2009, 406; Nagler u.a. 2010, 50-53; Gass 2011a, 68).

Ähnliche „Straßen“ wurden noch bei I8 weiteren Kurganen im südöstlichen Siebenstromland festgestellt (Tabelle I7).

Die sog. Prozessionswege wurden um i7 Groß- (5\% aller untersuchten Großkurgane) und um zwei Kleinkurgane (O,3\% aller untersuchten Kleinkurgane) (Gf. am Stausee Bartogaj, Nordostufer K6326 und Gf. Žylysaj-I, K3327) errichtet. Großkurgane mit sog. Prozessionswegen kommen in allen Größenkategorien vor: sieben große Kurgane $(2-4 \mathrm{~m})^{328}$, acht größere Kurgane $(4-8 \mathrm{~m})^{329}$ und zwei sehr große Kur-

$3222 \%$ aller untersuchten Kurgane im südöstlichen Siebenstromland.

323 Siehe Katalog, Gf. Žoan Tobe, Kurgancharakteristik, Kurgan I.

324 Für Informationen zur Untersuchung der Objekte 3I-Nord und 3I-Ost beim Kurgan I auf dem Gf. Žoan Tobe im Jahre 2008 möchte ich mich bei H. Parzinger, Z. Samašev, A. Nagler, M. Nawroth und M.-R. Boroffka herzlich bedanken.
325 «Ритуальная дорога».

$326 \mathrm{H}=0,7 \mathrm{~m}$; Dm=20 m; siehe Katalog, Gf. am Stausee Bartogaj, Kurgancharakteristik.

$327 \mathrm{H}=\mathrm{I}, 5 \mathrm{~m}$; Dm=I7 m; siehe Katalog, Gf. Žylysaj-I, Kurgancharakteristik.

$32837 \%$ aller Kurgane mit sog. Prozessionsweg.

$32943 \%$ aller Kurgane mit sog. Prozessionsweg. 
Tabelle 17 | Kurgane mit sog. Prozessionswegen

\begin{tabular}{|c|c|c|c|c|c|c|c|c|}
\hline 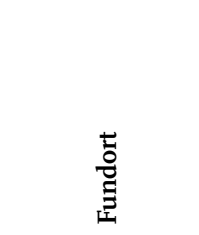 & $\frac{\frac{\pi}{2}}{\frac{0}{0}}$ & 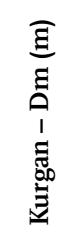 & 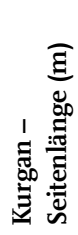 & 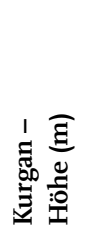 & 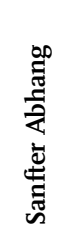 & 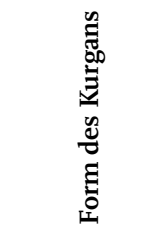 & 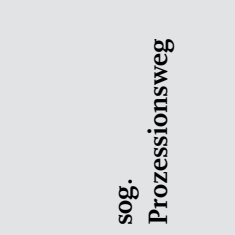 & 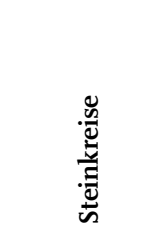 \\
\hline Gf. Aksaj-9 & K8 & $3 \mathrm{I}$ & & 3 & S & abgerundet & $\mathrm{Dm}=52 \mathrm{~m} ; \mathrm{B}=\mathrm{Im}$ & \\
\hline Gf. Aktasty-3 & $\mathrm{K}_{4}$ & 25 & & 2,5 & S & abgerundet & $\begin{array}{l}\mathrm{Dm}=39 \mathrm{~m} ; \\
\mathrm{B}=0,5-0,7 \mathrm{~m}\end{array}$ & \\
\hline Gf. Asy Saga & $\begin{array}{l}\mathrm{KI} \\
\mathrm{K}_{3} \\
\mathrm{~K}_{4}\end{array}$ & $\begin{array}{l}\mathrm{I} 49 \\
62\end{array}$ & 56 & $\begin{array}{l}2 \mathrm{I} \\
4 \\
5\end{array}$ & $\begin{array}{l}\text { S } \\
\text { S } \\
\text { SW }\end{array}$ & $\begin{array}{l}\text { abgerundet } \\
\text { viereckig } \\
\text { abgerundet }\end{array}$ & $\begin{array}{l}\mathrm{Dm}=252 \mathrm{~m} ; \mathrm{B}=5 \mathrm{~m} \\
\text { Seitenlänge }=95 \mathrm{~m} ; \\
B=2,5 \mathrm{~m} \\
\mathrm{Dm}=106 \mathrm{~m} ; \mathrm{B}=2 \mathrm{~m}\end{array}$ & \\
\hline $\begin{array}{l}\text { Gf. am Bartogaj } \\
\text { Stausee }\end{array}$ & $\begin{array}{l}\text { Nordostu- } \\
\text { fer - K6 }\end{array}$ & 20 & & 0,7 & $\begin{array}{l}\text { kein- } \\
\text { e }\end{array}$ & viereckig & $\begin{array}{l}\text { Dm ca. } 36-4 \mathrm{Im} ; \\
\mathrm{B}=\mathrm{I}, \mathrm{O}-\mathrm{I}, 5 \mathrm{~m}\end{array}$ & \\
\hline Gf. Kyzylšaryk & $\begin{array}{l}\text { Kio } \\
\text { KI4 }\end{array}$ & $\begin{array}{l}60 \\
56\end{array}$ & & $\begin{array}{l}7 \\
7\end{array}$ & $\begin{array}{l}\mathrm{SW} \\
\mathrm{S}\end{array}$ & $\begin{array}{l}\text { abgerundet } \\
\text { abgerundet }\end{array}$ & $\begin{array}{l}\mathrm{Dm}=86 \mathrm{~m} ; \mathrm{B}=2 \mathrm{~m} \\
\mathrm{Dm}=86 \mathrm{~m} ; \mathrm{B}=\mathrm{I}, 5 \mathrm{~m}\end{array}$ & \\
\hline Gf. Turgen & $\begin{array}{l}\mathrm{K}_{3} \\
\mathrm{~K}_{4}\end{array}$ & $\begin{array}{l}70 \\
5 \mathrm{I}\end{array}$ & & $\begin{array}{l}7 \\
6,5\end{array}$ & $\begin{array}{l}\text { S } \\
\text { S }\end{array}$ & $\begin{array}{l}\text { abgerundet } \\
\text { abgerundet }\end{array}$ & $\begin{array}{l}\mathrm{Dm}=139 \mathrm{~m} ; \mathrm{B}=3 \mathrm{~m} \\
\mathrm{Dm}=84 \mathrm{~m} ; \mathrm{B}=2 \mathrm{~m}\end{array}$ & $\begin{array}{l}\text { Ja } \\
(\mathrm{Dm}=200 \mathrm{~m})\end{array}$ \\
\hline Gf. Žalauly-2 & K6I & 37 & & 4 & S & abgerundet & $\begin{array}{l}\mathrm{Dm}=62 \mathrm{~m} \text { (rekon- } \\
\text { struiert); B=I,5m }\end{array}$ & \\
\hline Gf. Žoan Tobe & KI & II3 & & II & $\mathrm{S}$ & abgerundet & $\begin{array}{l}\mathrm{Dm}=185 \mathrm{~m} \\
\mathrm{~B}=3,0-3,5 \mathrm{~m}\end{array}$ & \\
\hline Gf. ŽYlysaj-1 & $\begin{array}{l}\mathrm{K}_{2} \\
\mathrm{~K}_{3} \\
\mathrm{~K}_{5} \\
\mathrm{~K} 6 \\
\mathrm{~K}_{7} \\
\mathrm{~K} 9 \\
\mathrm{KII}\end{array}$ & $\begin{array}{l}39 \\
21 \\
27\end{array}$ & 28 & $\begin{array}{l}4 \\
\mathrm{I}, 5 \\
3,5 \\
3 \\
3 \\
2 \\
3\end{array}$ & $\begin{array}{l}\mathrm{S} \\
\mathrm{S} \\
\mathrm{S} \\
\mathrm{S} \\
\mathrm{S} \\
\mathrm{S} \\
\mathrm{S}\end{array}$ & $\begin{array}{l}\text { abgerundet } \\
\text { abgerundet } \\
\text { abgerundet } \\
\text { viereckig } \\
\text { abgerundet } \\
\text { abgerundet } \\
\text { abgerundet }\end{array}$ & $\begin{array}{l}\mathrm{Dm}=50 \mathrm{~m} ; \mathrm{B}=\mathrm{Im} \\
\mathrm{Dm}=28 \mathrm{~m} ; \mathrm{B}=\mathrm{Im} \\
\mathrm{Dm}=49 \mathrm{~m} ; \mathrm{B}=\mathrm{Im} \\
\text { Seitenlänge=45m; } \\
\mathrm{B}=\mathrm{Im} \\
\mathrm{Dm}=5 \mathrm{Im} ; \mathrm{B}=\mathrm{Im} \\
\mathrm{Dm}=42 \mathrm{~m} ; \mathrm{B}=\mathrm{Im} \\
\mathrm{Dm}=4 \mathrm{Im} ; \mathrm{B}=\mathrm{Im}\end{array}$ & \\
\hline
\end{tabular}

gane330, deren Höhe acht Meter übertrifft (Gf. Asy Saga, KI331 und Gf. Žoan Tobe, KI332). Die Kombination von Kurgan und sog. Prozessionsweg tritt in allen Verbreitungszonen der Gräberfelder mit großen fürstlichen Kurganen der Saken im Forschungsgebiet auf. Fast alle Kurgane mit sog. Prozessionsweg hatten drei steile und einen sanften Abhang. Bei i6 Kurganen wurde der sanfte Abhang exakt nach Süden $(84 \%$ aller Kurgane mit einem sog. Prozessionsweg) und bei zwei weiteren Tumuli nach Südwesten (II\%) ausgerichtet. Die Aufschüttung eines Kurgans war sehr gleichmäßig errichtet und zeigte 

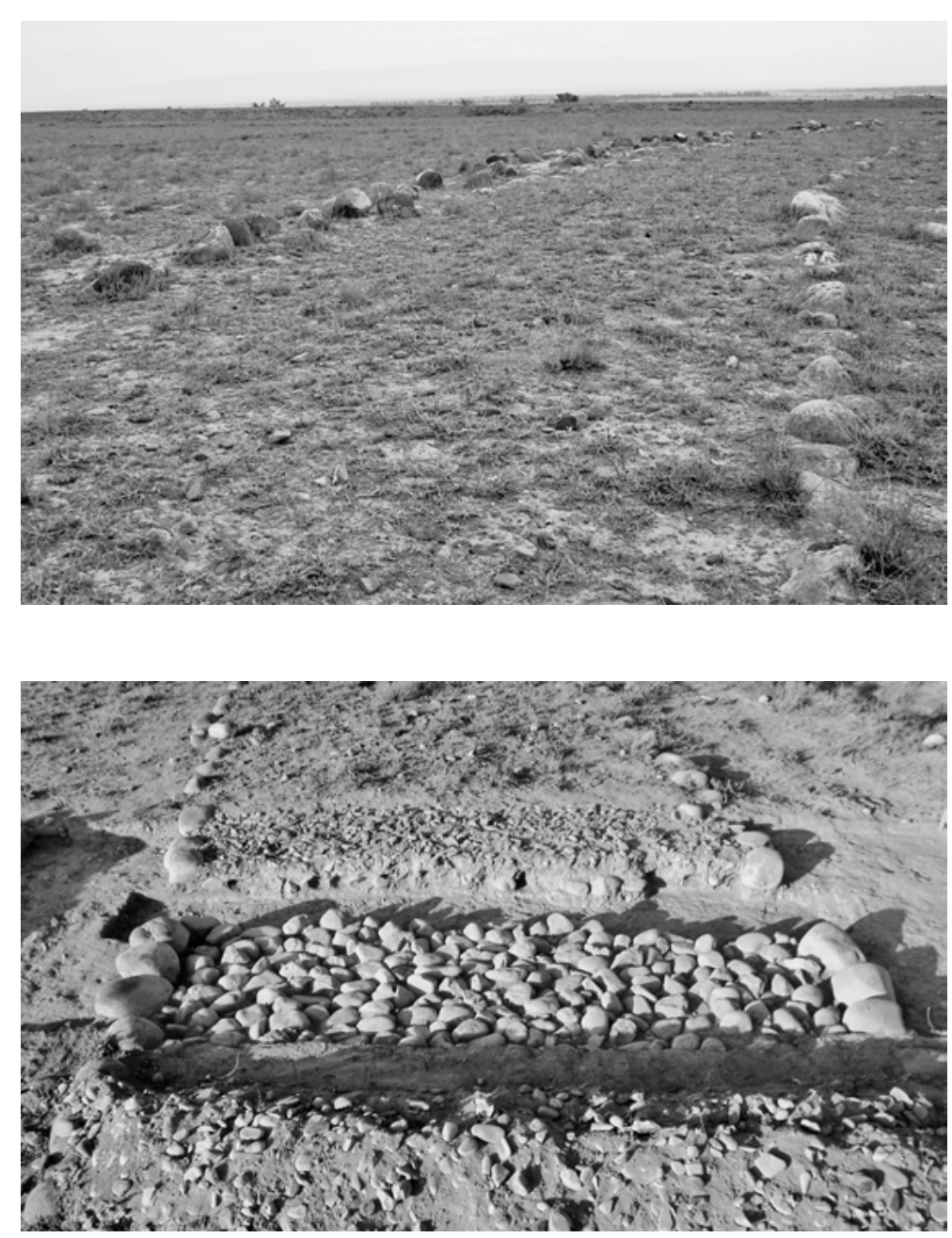

Abb. ו | Sog. Prozessionsweg. Gf. Asy Saga. Kurgan I. Südwestlicher Bereich. Blick von O
Abb. 72 | Sog. Prozessionsweg. Gf. Žoan Tobe. Kurgan I. Objekt 3I-Ost. Blick von SW (Samašev u.a. 2009, Abb. 2)

keine solche Merkmalet333. In drei Fällen (Gf. Asy Saga, K3334; Gf. am Stausee Bartogaj Nordostufer, K6335 und Gf. Žylysaj-I, K6336) wies die Kurganaufschüttung eine viereckige, pyramidenähnliche Form mit abgeflachter Kuppe auf. Die restlichen I6 Kurgane hatten eine abgerundete Form mit abgeflachter Kuppe.

Bei neun Kurganen auf insgesamt sechs Gräberfeldern wurden zwischen dem Rand und dem sog. Prozessionsweg zusätzlich die Peripheriekonstruktionen festgestellt. Außerhalb des sog. Prozessionsweges von Kurgan 3 des Gräberfeldes Turgen337 befand sich ein Steinkreis.

Die äußere Markierung der sog. Prozessionswege um den Kurgan I des Gräberfeldes Asy Saga338 und um die Kurgane 3 und 4 der Nekropole Turgen339 bestand ausschließlich aus rötlichen, mittelgroßen und großen Steinen $(60-50 \times 40 \times 30 \mathrm{~cm})$.

333 K6 am Nordostufer des Gf. am Stausee Bartogaj; siehe Katalog, Gf. am Stausee Bartogaj, Kurgancharakteristik.

334 Siehe Katalog, Gf. Asy Saga, Kurgancharakteristik.

335 Siehe Katalog, Gf. am Stausee Bartogaj, Kurgancharakteristik.
336 Siehe Katalog, Gf. Žylysaj-I, Kurgancharakteristik.

337 Siehe Katalog, Gf. Turgen, Kurgancharakteristik.

338 Siehe Katalog, Gf. Asy Saga, Kurgancharakteristik.

339 Siehe Katalog, Gf. Turgen, Kurgancharakteristik. 
Tabelle 18 | Verhältnis von Kurgandurchmesser zum

Durchmesser des sog. Prozessionsweges

\begin{tabular}{|c|c|c|}
\hline $\begin{array}{l}\text { Kurgan-Dm } \\
\text { bzw. Seiten- } \\
\text { länge (m) }\end{array}$ & $\begin{array}{l}\text { Dm des sog. } \\
\text { Prozessions- } \\
\text { weges }(\mathrm{PW})(\mathrm{m})\end{array}$ & $\begin{array}{l}\text { Verhältnis der } \\
\varnothing \text { vom Kurgan } \\
\text { zum PW }\end{array}$ \\
\hline $3 \mathrm{I}$ & 52 & 0,596 \\
\hline 25 & 39 & 0,64 \\
\hline I49 & 252 & $0,59^{\mathrm{I}}$ \\
\hline 56 & 95 & 0,589 \\
\hline 62 & 106 & 0,584 \\
\hline 20 & 36 & 0,555 \\
\hline 60 & 86 & o,697 \\
\hline 56 & 86 & $0,65 \mathrm{I}$ \\
\hline 70 & I39 & 0,504 \\
\hline $5^{\mathrm{I}}$ & 84 & 0,607 \\
\hline 37 & 62 & 0,596 \\
\hline II3 & I85 & $0,6 \mathrm{II}$ \\
\hline 37 & 50 & 0,74 \\
\hline I7 & 28 & 0,607 \\
\hline $3 \mathrm{I}$ & 49 & 0,632 \\
\hline 28 & 45 & 0,622 \\
\hline 39 & $5^{\mathrm{I}}$ & 0,764 \\
\hline $2 \mathrm{I}$ & $4^{2}$ & 0,5 \\
\hline 27 & $4 \mathrm{I}$ & 0,658 \\
\hline \multicolumn{2}{|c|}{ Mittelwert: } & $0,6 \mathrm{I} 8$ \\
\hline
\end{tabular}

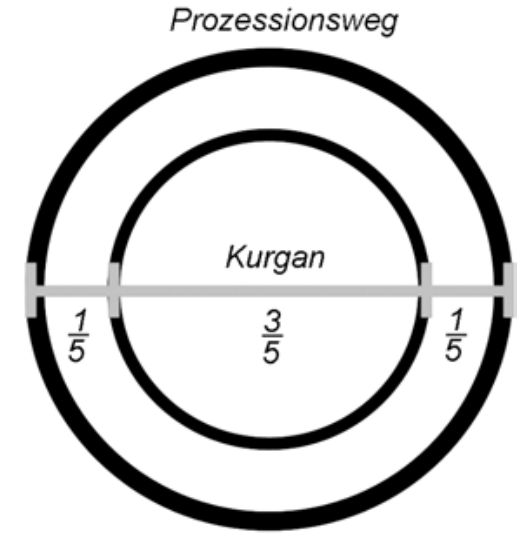

Abb. 73 | Graphische Darstellung des proportionalen Verhältnisses von Kurgandurchmesser zu sog. Prozessionsweg (ohne Maßstab)

Die aufgenommenen sog. Prozessionswege bei den sakischen Kurganen im südöstlichen Siebenstromland hatten einen äußeren Durchmesser von $2834^{\circ}$ bis $25^{2} \mathrm{~m}^{34 \mathrm{I}}$ und wiesen eine Breite von o,5 bis 5, $0 \mathrm{~m}$ auf. Der Durchmesser der sog. Prozessionswege 342 stand in direktem Verhältnis zum Durchmesser des Kurgans343 (Tabelle I8). Betrachtet man den Durchmesser von Kurgan und sog. Prozessionsweg als Einheit, misst der Durchmesser eines Kurgans immer $3 / 5$ und der Abstand zwischen dem Kurganrand und dem sog. Prozessionsweg I/5 des Konstruktionsdurchmessers344 (Abb. 73). Diese Tatsache betrifft so-

340 Kleinster sog. Prozessionsweg - Gf. Žylysaj-I K3, siehe Tabelle I7, vgl. Katalog, Gf. Žylysaj-I, Kurgancharakteristik. 34I Größter sog. Prozessionsweg - Gf. Asy Saga KI, siehe Tabelle I7, vgl. Katalog, Gf. Asy Saga, Kurgancharakteristik.

342 Von der Außenseite gemessen.

343 Hierbei muss jedoch beachtet werden, dass gewisse Abweichungen beim Messen der Durchmesser von Kurgan und sog. Prozessionsweg entstehen können, da der heutige Durchmesser nicht unbedingt dem ursprünglichen entsprechen muss. Der ursprüngliche Durchmesser kann durch Verwitterungsprozesse der Kurganaufschüttung verändert worden sein.
344 Nimmt man an, dass der Durchmesser eines Kurgans $a$ und der Durchmesser des sog. Prozessionsweges $b$ ist, kommt man beim Ausrechnen des Verhältnisses von Kurgandurchmessern zu sog. Prozessionsweg auf die Formel der "Gleichungen “ $a \div b=x \div c$, wobei $x$ - das unbekannte Verhältnis und $c$ - die ganze Konstruktion in der Proportion ist. Die Proportion des Durchmessers des Kurgans zu dem Durchmesser des sog. Prozessionsweges kann durch Kreuzmultiplikation in eine Gleichung der Form $a \cdot x=b \cdot c$ gebracht werden (Gellert u.a. I979, 447). Da die gesamte Konstruktion in der Proportion 1 gleicht, erfolgt dann die Form $a \cdot x=b \cdot 1$. 
wohl die abgerundeten als auch die viereckigen Kurgane. Demzufolge spielte offensichtlich nicht das Ausmaß eines Kurgans, sondern die Gesamtproportion eine Rolle.

Im zentralen Bergaltai fand man um einige Großkurgane besondere Steinkonstruktionen vor345. Es handelte sich um doppelte Steinkreise, die im Innenraum gepflastert worden sind. Direkt am Rand der Kurgane Bašadar-I (Rudenko I960, 26) und Bašadar-2 (Rudenko I960, 30) befand sich ein Steinkreis aus senkrecht stehenden Steinen. In einem Abstand von $4 \mathrm{~m}$ nach außen wurde ein zweiter Steinkreis errichtet. Im Zwischenraum wurde eine doppelte Pflasterschicht angelegt, so dass die Pflasterung dieselbe Höhe wie die Oberkante des Steinkreises erreichte. Ähnliche Doppelkreise aus senkrechtstehenden Steinen und der Pflasterung im Zwischenraum wurden auch bei den Großkurganen Tuekta-I und Tuekta-2 errichtet (Rudenko I960, Io6, Abb. 53). Zudem wurden Doppelsteinkreise mit Innenpflasterung direkt am Kurganrand auch auf dem Plateau Ukok um den größten Kurgan des Gräberfeldes Kuturguntas festgestellt (Polos'mak 200I, IOI). Eine ähnliche Konstruktion wies ferner der „Fürstenkurgan“ in Sentelek auf, mit dem Unterschied, dass dort der innere Steinkreis direkt im Kurganpanzer integriert wurde und die Pflasterung der Zwischenräume nicht die Höhe der Oberkanten der senkrechtstehenden Steine erreichte (Šul'ga 20IO, Abb. I). Eine derartige Konstruktion, wie um den Kurgan in Sentelek, wurde von den Ausgräbern als ein „Korridor“ bezeichnet (Kubarev/Šul'ga 2007, 47).

Die „Korridore“ um die Kurgane vom Bergaltai weisen eine gewisse Ähnlichkeit zu den sog. Prozessionswegen um die Kurgane im südöstlichen Siebenstromland auf. Mit größter Wahrscheinlichkeit hatten diese ähnlichen Konstruktionen auch dieselbe Bedeutung. Allerdings sind hier sowohl die Unterschiede in der Bautechnik als auch in der Platzierung dieser Befunde um einen Kurgan anzumerken.

Die sog. Prozessionswege der sakischen Kurgane im südöstlichen Siebenstromland zeigen eine größere Ähnlichkeit zu einer gepflasterten Straße und befinden sich immer in einem streng reglementierten Abstand vom Kurganrand. Damit sind die sog. Prozessionswege um sakische Kurgane im gesamten skytho-sakischen Kulturkreis Eurasiens einmalig.

\subsubsection{Kreisgräben}

Auf sechs Nekropolen wurden Kurgane mit umlaufendem Kreisgraben festgestellt, die heute noch an der Oberfläche sichtbar sind (Abb. 74). Es handelt sich um insgesamt 28 Grabhügel346 (Tabelle I9; Karte I4). Davon können neun Kurgane als Klein- (I,5\% aller untersuchten Kleinkurgane) und die restlichen I9 als Großkurgane ( $6 \%$ aller untersuchten Großkurgane) bezeichnet werden. Großkurgane mit Kreisgraben sind unter allen Größenkategorien zu finden, darunter sieben große Kurgane (2-4 m) ${ }^{347}$, neun größere Kurgane (4-8 m) 348 $^{8}$ und drei sehr große Kurgane mit Kreisgraben349 (Höhe über 8 m). Kurgane mit Kreisgraben treten in allen Verbreitungszonen der Gräberfelder mit großen fürstlichen

Die Ergebnisse sind in Tabelle I8 dargestellt. Rechnet man den Mittelwert aus und rundet die Ergebnisse, bekommt man schlussendlich einen Wert von o,6. Was bei einer Umwandlung einer Dezimalzahl in eine Bruchzahl 3/5 ergibt. 3/5 stellt den Anteil des Kurgandurchmessers von der gesamten Konstruktion dar.

345 Für die freundlichen Hinweise bezüglich der Peripherie der Großkurgane im Bergaltai bedanke ich mich herzlich beim Dr. P. I. Šul'ga, Barnaul (Russland).
$3463 \%$ aller im südöstlichen Siebenstromland untersuchten Kurgane.

$34725 \%$ aller Kurgane mit Kreisgraben.

$348 \quad 32 \%$ aller Kurgane mit Kreisgraben.

349 II \% aller Kurgane mit Kreisgraben. Gf. Boroldaj, K5, Gf. auf dem Plateau Kegen, K4 und Gf. Togyzbulak, KI (siehe Katalog, dementsprechende Gräberfelder, Kurgancharakteristik). 
Abb. 74 | Kurgan 5 km südwestlich vom Dorf Žalauly. Westseite des Kreisgrabens. Eingangsbereich. Blick vom Kurgan, von O

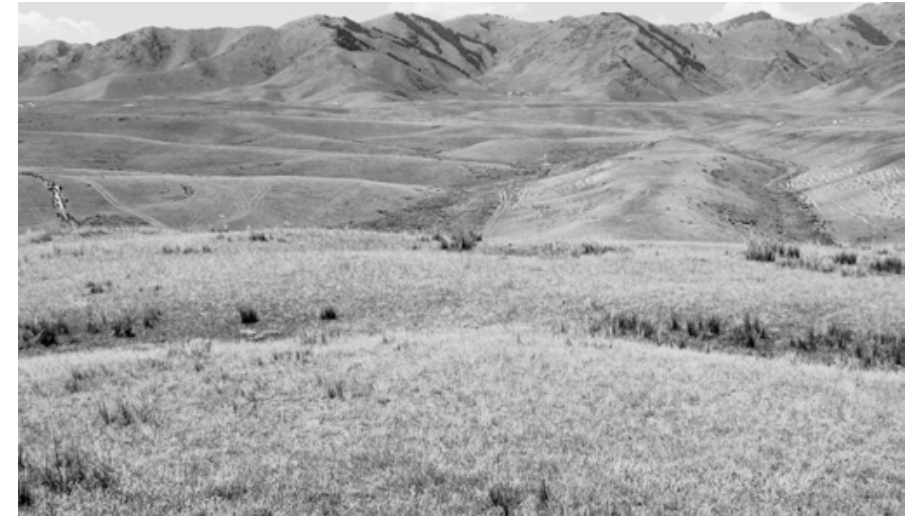

Kurganen der Saken im Forschungsgebiet auf. Nur bei I7 Kurganen (6r\% aller Kurgane mit einem Kreisgraben) konnte festgestellt werden, dass die Kurganaufschüttung drei steile und einen sanften Abhang hatte. Die restlichen elf Kurgane zeigten diese Merkmale entweder gar nicht oder ihre Oberfläche war so stark zerstört bzw. beschädigt, dass dort diesbezüglich keine Aussagen getroffen werden konnten. Bei neun von diesen I7 Kurganen wurde der sanfte Abhang exakt nach Süden ausgerichtet (32\% aller Kurgane mit einem Kreisgraben). Jeweils drei Kurgane (je Iı \%) wiesen einen sanften Abhang nach Südosten bzw. Westen auf. In nur einem Fall wies ein solcher Abhang nach Südwesten bzw. Nordwesten. Fast alle untersuchten Kurgane haben eine abgerundete Form mit abgeflachter Kuppe. Nur ein Grabhügel stellte eine Ausnahme dar, nämlich der Kurgan 5 km südwestlich vom Dorf Žalauly. Die Aufschüttung ist hier von viereckiger, pyramidenartiger Form mit abgeflachter Kuppe.

Zwischen dem Kurganrand und dem Kreisgraben wurde ein Kleinkurgan dokumentiert. Er zählt zur Kategorie Peripheriekonstruktionen. Die Anlage eines Kleinkurgans zwischen Großkurgan und Kreisgraben ist nur in diesem einen Fall nachgewiesen.

Um den Kurgan 25 des Gräberfeldes Boroldaj350 wurde ein Steinkreis außerhalb des Kreisgrabens gebaut. Ebenso auf der Nekropole Boroldaj wurden um den Rand von Kurgan I035ז zunächst ein Kreisgraben, dann ein Wall und noch ein Kreisgraben errichtet (Gass 20I2, 47I). Alle anderen Kurgane mit einem Kreisgraben wiesen gar keine weiteren Konstruktionen in ihrer Peripherie auf. Eine Kombination aus Graben und sog. Prozessionsweg wurde bis jetzt ebenfalls nicht festgestellt.

Was das Ausmaß der Kreisgräben betrifft, die an der modernen Oberfläche sichtbar sind, kann folgendes vermerkt werden. Die Gräben haben einen Durchmesser von Io m bis zu $223 \mathrm{~m}$, ihre Breite schwankt zwischen 0,5-0,7 $\mathrm{m}$ und $24 \mathrm{~m}$, die Tiefe beträgt $0,2 \mathrm{~m}$ bis zu 2,0-2,5 m. Der Abstand zwischen dem Kreisgraben und dem Rand des Kurgans variiert zwischen einigen Zentimeter bis zu $67 \mathrm{~m}$.

Bei acht Kurganen sind die Kreisgräben unterbrochen, so dass Eingangsbereiche entstanden sind (siehe Tabelle I9). Davon wurde bei fünf Kurganen nur ein Eingangsbereich festgestellt. In drei Fällen befand er sich in der Westhälfte der Konstruktion $35^{2}$ und jeweils einmal trat der Eingangsbereich exakt im Süden353 bzw. im Südwesten354 auf. Die Kreisgräben um drei weitere Kurgane wiesen zwei Eingangs-

Siehe Katalog, Gf. Boroldaj, Kurgancharakteristik.

$\begin{array}{ll}35 \mathrm{I} & \text { Siehe Anm. 350. } \\ 352 & \text { Kurgan } 5 \mathrm{~km} \text { südwestlich vom Dorf Žalauly, Gf. Borol- }\end{array}$ daj, Kurgane 32 und 33; siehe Katalog, Kurgan 5 km südwestlich vom Dorf Žalauly und Gf. Boroldaj, Kurgancharakteristik.
353 Gf. Boroldaj, Ki5; siehe Katalog, Gf. Boroldaj, Kurgancharakteristik.

354 Gf. Boroldaj, K7; siehe Katalog, Gf. Boroldaj, Kurgancharakteristik. 
Tabelle 19 | Kurgane mit einem Kreisgraben

\begin{tabular}{|c|c|c|c|c|c|c|c|c|}
\hline 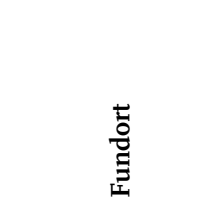 & $\frac{\pi}{\frac{\pi}{0}}$ & 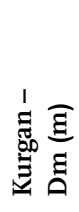 & 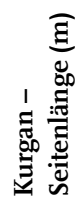 & 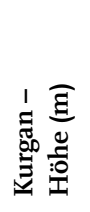 & 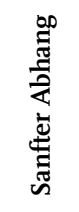 & 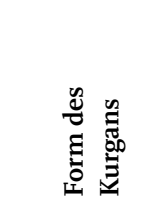 & 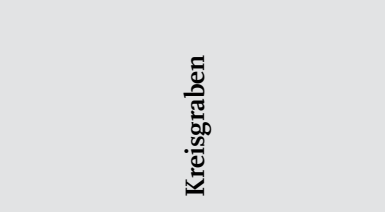 & 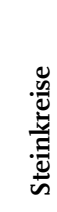 \\
\hline $\begin{array}{l}\text { Ein Kurgan } 5 \mathrm{~km} \\
\text { südwestlich vom } \\
\text { Dorf Žalauly }\end{array}$ & $\mathrm{IK}_{5} \mathrm{SW}$ & & 28 & 3 & & viereckig & $\begin{array}{l}\mathrm{Dm}=73 \mathrm{~m} ; \mathrm{B}=\mathrm{Iom} ; \mathrm{T}=\mathrm{Im} ; \\
\text { W-Hälfte Eingangsbereich }\end{array}$ & \\
\hline \multirow[t]{18}{*}{ Gf. Boroldaj } & KI & 39,7 & & 3,4 & $\mathrm{~W}$ & abgerundet & $\mathrm{Dm}=4 \mathrm{I}, 7 \mathrm{~m} \mathrm{~B} \circ, 7-\mathrm{I}, \circ \mathrm{m} ; \mathrm{T}$ ? & \\
\hline & $\mathrm{K}_{5}$ & 83 & & 9,4 & keine & abgerundet & $\begin{array}{l}\mathrm{Dm}=85 \mathrm{~m} ; \mathrm{B} \text { I,o } \mathrm{m} ; \mathrm{T} \text { ?; } \mathrm{W}+\mathrm{SW} \\
\text { zwei Eingangsbereiche }\end{array}$ & \\
\hline & $\mathrm{K}_{7}$ & 52,2 & & 6,8 & NW & abgerundet & $\begin{array}{l}\mathrm{Dm}=54,5 \mathrm{~m}, \mathrm{~B} \text { ०,5-I,o } \mathrm{m} ; \mathrm{T} \text { ?; } \\
\mathrm{SW} \text { ein Eingangsbereich }\end{array}$ & \\
\hline & K8 & 30 & & $\mathrm{I}, 6$ & $\mathrm{~S}$ & abgerundet & $\mathrm{B}$ ?; $\mathrm{T}$ ? & \\
\hline & K9 & 37,8 & & 4 & SO & abgerundet & $\mathrm{B}$ ?; $\mathrm{T}$ ? & \\
\hline & KIO & 44,8 & & 5 & $\mathrm{SO}$ & abgerundet & $\begin{array}{l}\text { Graben-Wall-Graben; } \\
\text { B I,5 m; T ०,5-I,० m }\end{array}$ & \\
\hline & KI5 & 32,5 & & 2,4 & keine & abgerundet & $\begin{array}{l}\mathrm{Dm}=34,5 \mathrm{~m} ; \mathrm{B} \text { I, } \mathrm{o} \mathrm{m} ; \mathrm{T} \text { ?; } \\
\mathrm{S} \text { ein Eingangsbereich }\end{array}$ & \\
\hline & KI7 & 36,5 & & 2,2 & S & abgerundet & $\mathrm{B}$ o,5-0,7 m; T ? & \\
\hline & Кı 8 & $3 \mathrm{I}, 6$ & & I,4 & SO & abgerundet & $\mathrm{B}$ ?; $\mathrm{T}$ ? & \\
\hline & $\mathrm{K} 24$ & $7 \mathrm{I}, 9$ & & 7,6 & S & abgerundet & $\mathrm{Dm}=74 \mathrm{~m} ; \mathrm{B}$ Im; $\mathrm{T} \circ, 3 \mathrm{~m}$ & \\
\hline & $\mathrm{K} 25$ & 83,5 & & 6 & SW & abgerundet & $\mathrm{B}$ ?; $\mathrm{T}$ ? & $\mathrm{x}$ \\
\hline & $\mathrm{K} 26$ & $5 \mathrm{I}, 5$ & & 4,7 & W & abgerundet & $\mathrm{B}$ ?; $\mathrm{T}$ ? & \\
\hline & $\mathrm{K} 28$ & 46,2 & & $?$ & $\mathrm{~S}$ & abgerundet & $\mathrm{B}$ ?; $\mathrm{T}$ ? & \\
\hline & $\mathrm{K}_{3} \mathrm{O}$ & 33 & & 3,6 & $?$ & abgerundet & B o,7-I,० m; T ? & \\
\hline & $\mathrm{K}_{3} \mathrm{I}$ & 20 & & $\mathrm{I}, 5$ & $?$ & abgerundet & $\mathrm{B} \circ, 7 \mathrm{~m} ; \mathrm{T}$ ? & \\
\hline & $\mathrm{K}_{32}$ & $3 \mathrm{I}, 3$ & & 2 & ? & abgerundet & B ?; $\mathrm{T}$ ?; W ein Eingangsbereich & \\
\hline & $\mathrm{K}_{33}$ & $34, \mathrm{I}$ & & 2,5 & $\mathrm{~W}$ & abgerundet & $\mathrm{B}$ ?; $\mathrm{T}$ ?; $\mathrm{W}$ ein Eingangsbereich & \\
\hline & $\mathrm{K}_{4} \mathrm{O}$ & 40 & & 4 & $\mathrm{~S}$ & abgerundet & $\mathrm{B}$ ?; T ? & \\
\hline Gf. Ereul & $\mathrm{K}_{35}$ & 54 & & 4,5 & $\mathrm{~S}$ & abgerundet & $\mathrm{Dm}=\mathrm{II} 4 ; \mathrm{B}=\mathrm{IIm} ; \mathrm{T}=\mathrm{I}, \mathrm{O}-\mathrm{I}, 5 \mathrm{~m}$ & \\
\hline $\begin{array}{l}\text { Gf. auf dem } \\
\text { Plateau Kegen }\end{array}$ & $\mathrm{K}_{4}$ & 85 & & 9 & $\mathrm{~S}$ & abgerundet & $\begin{array}{l}\mathrm{Dm}=\mathrm{I} 93 \mathrm{~m} ; \mathrm{B}=\mathrm{I} 5,5 \mathrm{~m} ; \\
\mathrm{T}=2, \mathrm{O}-2,5 \mathrm{~m} ; \mathrm{W} \text { - und } \\
\text { O-Hälften 8-Iom breite Ein- } \\
\text { gangsbereiche }\end{array}$ & \\
\hline Gf. Togyzbulak & KI & 89 & & IO & $\mathrm{S}$ & abgerundet & $\begin{array}{l}\mathrm{Dm}=223 \mathrm{~m} ; \mathrm{B}=24 \mathrm{~m} \text {; } \\
\mathrm{T}=2, \mathrm{O}-2,5 \mathrm{~m} \text {; W-Eingangs- } \\
\text { bereich: I3m; O-Eingangs- } \\
\text { bereich: } 9 \mathrm{~m}\end{array}$ & \\
\hline \multirow[t]{6}{*}{ Gf. Žalauly-8 } & $\mathrm{K}_{2}$ & IO & & 0,5 & keine & abgerundet & $\mathrm{Dm}=\mathrm{I} 4 \mathrm{~m} ; \mathrm{B}=2 \mathrm{~m} ; \mathrm{T}=0,5 \mathrm{~m}$ & \\
\hline & $\mathrm{K}_{3}$ & 7 & & 0,5 & keine & abgerundet & $\mathrm{Dm}=\mathrm{IIm} ; \mathrm{B}=2 \mathrm{~m} ; \mathrm{T}=0,5 \mathrm{~m}$ & \\
\hline & $\mathrm{K}_{5}$ & Io & & 0,5 & keine & abgerundet & $\mathrm{Dm}=\mathrm{I} 4 \mathrm{~m} ; \mathrm{B}=2 \mathrm{~m} ; \mathrm{T}=0,5 \mathrm{~m}$ & \\
\hline & K6 & I4 & & $\mathrm{I}, 5$ & keine & abgerundet & $\mathrm{Dm}=\mathrm{I} 8 \mathrm{~m} ; \mathrm{B}=2 \mathrm{~m} ; \mathrm{T}=0,5 \mathrm{~m}$ & \\
\hline & $\mathrm{K}_{7}$ & 6 & & 0,3 & keine & abgerundet & $\mathrm{Dm}=\mathrm{IOm} ; \mathrm{B}=2 \mathrm{~m} ; \mathrm{T}=0,2 \mathrm{~m}$ & \\
\hline & $\mathrm{K}_{9}$ & 16 & & $\mathrm{I}, 5$ & S & abgerundet & $\mathrm{Dm}=22 \mathrm{~m} ; \mathrm{B}=3 \mathrm{~m} ; \mathrm{T}=0,5 \mathrm{~m}$ & \\
\hline
\end{tabular}


Tabelle 20 | Verhältnis des Kurgandurchmessers zum umgebenden Graben (nach Steigerung der Verhältniszahl angeordnet)

\begin{tabular}{|c|c|c|c|}
\hline Objekt & Kurgan Dm (m) & Kreisgraben Dm (m) & $\begin{array}{l}\text { Verhältnis der } \varnothing \\
\text { von Kurgan zu Graben }\end{array}$ \\
\hline${ }_{I} K_{5} S W$ & 28 & 73 & 0,38 \\
\hline Gf. Togyzbulak - KI & 89 & 223 & 0,40 \\
\hline Gf. Kegen - K4 & 85 & I93 & 0,44 \\
\hline Gf. Ereul - K35 & 54 & II4 & 0,47 \\
\hline Gf. Žalauly- $8-K_{7}$ & 6 & IO & 0,60 \\
\hline Gf. Žalauly- $8-K_{3}$ & 7 & II & 0,64 \\
\hline Gf. Žalauly- 8 - K2 & IO & I4 & $0,7 \mathrm{I}$ \\
\hline Gf. Žalauly- 8 - K5 & I0 & I4 & $0,7 \mathrm{I}$ \\
\hline Gf. Žalauly- 8 - Kg & 16 & 22 & 0,73 \\
\hline Gf. Žalauly- 8 - K6 & I4 & I8 & 0,78 \\
\hline Gf. Boroldaj - KIo & 44,8 & 48,2 & 0,93 \\
\hline Gf. Boroldaj - KI5 & 32,5 & 34,5 & 0,94 \\
\hline Gf. Boroldaj - KI & 39,7 & $4 \mathrm{I}, 7$ & 0,95 \\
\hline Gf. Boroldaj - K7 & 52,2 & 54,5 & 0,96 \\
\hline Gf. Boroldaj - K24 & 71,9 & 74 & 0,97 \\
\hline Gf. Boroldaj - K5 & 83 & 85 & 0,98 \\
\hline
\end{tabular}

bereiche auf. In zwei Fällen befanden sie sich auf den gegenüberliegenden Seiten (West-Ost) 355 und einmal im Westen und Südwesten der Konstruktion356. Die Breite dieser Eingänge betrug 8 bis I3 m (siehe Tabelle I9).

Das Verhältnis der Durchmesser von Kurgan zu Kreisgraben zeigte keine Regelhaftigkeit so wie es im Gegensatz dazu bei dem Verhältnis von Kurgan zu sog. Prozessionsweg der Fall ist. Trotzdem konnten einige sich wiederholende Tendenzen festgestellt werden. Wie die Tabelle 20 zeigt, unterscheidet sich dieses Verhältnis von Gräberfeld zu Gräberfeld. Innerhalb einer Nekropole jedoch sind diese Unterschiede nicht so gravierend und weisen nur minimale Schwankungen auf. So beträgt zum Beispiel das Verhältnis des Kurgandurchmessers zum Durchmesser eines Kreisgrabens auf dem Gräberfeld Žalauly-8 7:Io und auf der Nekropole Boroldaj 9,5:IO. Bei den sehr großen Kurganen, deren Höhe 8 m übersteigt, beträgt dieses Verhältnis 2:5.

Die Kreisgräben finden mehrere Parallelen im skytho-sakischen Kulturkreis. So ist zum Beispiel ein Kreisgraben um den Kurgan I des Gräberfeldes Bajkara nachgewiesen (Zajbert u.a. I999, I9; vgl. Parzinger u.a. 2003, 30-34, Abb. 36-39, Taf. 4, Beilage I-2, 6-7), gleichwohl um Kurgan I der Nekropole Javlenka (Chabdulina I994, Taf. II,I), um Kurgan 2 des Gräberfeldes Urnek (Chabdulina I994, Taf. 22,III), um Kurgan Kara-Oba (Chabdulina I994, Taf. 28) sowie um Kurgan Obaly (Chabdulina I994, Taf. 3०,Б) auf der Išim-Ebene in Nordkasachstan. Das Phänomen der Kreisgräben ist von den Kurganen 
der Sargat-Kultur im mittleren Irtyš- und Baraba-Tiefland Westsibiriens bekannt (Parzinger 2006, 57I, Abb. I85,26). Man fand sie ebenso bei den Kurganen im Altai wie z.B. um Kurgan II des Gräberfeldes Berel' (Samašev u.a. 2000, 8; Samašev 2007, Abb. 2). Ferner sind Kreisgräben für die Kleinkurgane Transnistriens357 (Ketrau/Četverikov 2003-2004, II9-I57, Abb. 27,I, 35,I, 38, 44,I) und für die großen fürstlichen Kurgane des mittleren Dnepr-Gebietes in der Ukraine dokumentiert (Slonovskaja Bliznica: Bliznicy Slonovskie I872, 64; Tolstaja Mogila: Mozolevs'kij ı979, I53; Vodjana Mogila: Mozolevskij/Polin 2005, 78-79, Abb. 4-5, II; Babina Mogila: Mozolevskij/Polin 2005, I04-I07, Abb. 27-28, 43; Soboleva Mogila: Mozolevskij/Polin 2005, I50, Abb. 77, 79-8I; Oguz: Boltrik/Fialko 2007, 269, Abb. I oder Aleksandropol'-Kurgan: Aleksandropol'skij kurgan I866, I-2; Polin 2007, 258-259; Polin/Daragan 2008, I50-I55, Abb. I-2; Polin/Daragan 20II, I93, Abb. 8-IO). In den Kreisgräben der Ukraine358 und Transnistriens 359 wurden stets Überreste der Trizna 360 gefunden, wie etwa Tierknochen von Haus- und Wildtieren, in einigen Fällen sogar Teilskelette bzw. vollständige Skelette vom Pferd, Fragmente griechischer Amphoren, griechische Import- und einheimische, skythische Keramik, Pfeilspitzen, Teile der Pferdeausrüstung, Bronzeschmuck vom Totenwagen und sogar einzelne Menschenknochen, die als Überreste von Opferhandlungen gedeutet werden konnten (Ketrau/Četverikov 2003-2004, II9, I32, I37, I48, Abb. 28, 37; Mozolevskij/Polin 2005, 78-8I, I50, 294-298; Polin 2007, 259; Polin/Daragan 2008, I56-I6o, Abb. 3).

Es wird angenommen, dass mit der Anlage des Steinkreises und dem Markieren der Eingangsbereiche der Bau des gesamten Kurgans begann (Parzinger u.a. 2003, 30; vgl. Mozolevskij/Polin 2005, 296). Möglicherweise schaffte man eine erste vorläufige Markierung mit einem einfachen Zirkel, der aus einem Seil und ein oder zwei Pflöcken bestand. So entstand dann ein magischer Kreis, der die Welt der Toten und der Lebenden voneinander trennte. In diesem rituellen Raum erfolgten die nächsten Schritte zum Bau des Kurgans (Ol'chovskij I99I, I75; Mozolevskij/Polin 2005, 296-297).

\subsubsection{Steinkreise}

Bei 33 Kurganen36r auf neun unterschiedlichen Gräberfeldern (Tabelle 2I; Karte I5) wurde ein den Tumulus umgebender Steinkreis festgestellt (Abb. 75). Diese Feststellung konnt für zwölf Groß- (4\% aller untersuchten Großkurgane) und 2I Kleinkurgane (3\% aller untersuchten Kleinkurgane) getroffen werden. Großkurgane mit einem Steinkreis wiesen nur die zwei kleineren Größenkategorien von Großkurganen auf. Vier Grabhügel sind als große Kurgane $(2-4 \mathrm{~m})^{362}$ und acht als größere Kurgane $(4-8 \mathrm{~m})^{363}$ anzusprechen. In der Peripherie der sehr großen Kurgane, deren Höhe über $8 \mathrm{~m}$ misst, sind keinerlei Steinkreise belegt. Kurgane mit einem Steinkreis fand man in allen Verbreitungszonen der Gräberfelder mit großen fürstlichen Kurganen der Saken im Forschungsgebiet. Ohne Ausnahme hatten alle Kurgane eine abgerundete Form mit abgeflachter Kuppe. Bei is Kurganen (58\% aller Kurgane mit Steinkreis) wurde beobachtet, dass die Kurganaufschüttung drei steile und einen sanften Abhang aufwies.

357 Kurgane 18, 20, 2I und 44 bei der Stadt Dubăsari.

358 Eine vollständige Untersuchung der Kreisgräben um die Großkurgane fand nur in der Ukraine statt: hier wurden die Kurgane Vodjana Mogila, Soboleva Mogila und Aleksandropol' unter der Leitung von B. N. Mozolevskij und später S. V. Polin ausgegraben.

359

Die Kreisgräben um die Kurgane bei der Stadt Dubăsari wurden auch vollständig untersucht. Allerdings handelt es sich hier nur um Kleinkurgane, deren Höhe unter $2 \mathrm{~m}$ und deren Durchmesser unter $40 \mathrm{~m}$ blieb.

360 Leichenschmaus.

36I $3 \%$ aller untersuchten Kurgane im südöstlichen Siebenstromland.

362 I2 $\%$ aller Kurgane mit einem Steinkreis.

$36324 \%$ aller Kurgane mit einem Steinkreis. 
Tabelle 21 | Kurgane mit einem Steinkreis

\begin{tabular}{|c|c|c|c|c|c|c|c|c|}
\hline 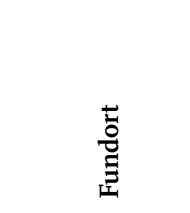 & $\frac{\frac{\pi}{0}}{\frac{0}{0}}$ & 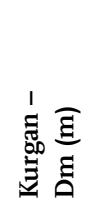 & 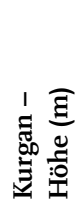 & 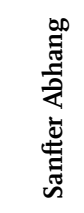 & 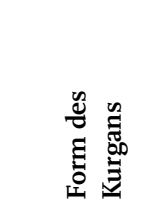 & 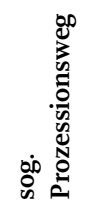 & 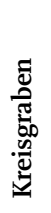 & 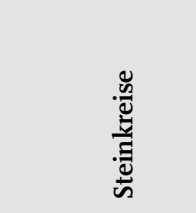 \\
\hline \multirow[t]{3}{*}{ Gf. Aksaj-9 } & $\mathrm{K}_{4}$ & $2 \mathrm{I}$ & $\mathrm{I}, 5$ & S & abgerundet & & & $\mathrm{Dm}=32 \mathrm{~m}$ \\
\hline & $\mathrm{K}_{7}$ & 28 & 2,2 & S & abgerundet & & & $\mathrm{Dm}=44 \mathrm{~m}$ \\
\hline & Kio & 22 & $\mathrm{I}, 5$ & $\mathrm{~S}$ & abgerundet & & & $\mathrm{Dm}=29 \mathrm{~m}$ \\
\hline \multirow[t]{6}{*}{ Gf. Aktasty-3 } & KI & 25 & $\mathrm{I}, 5$ & $\mathrm{~S}$ & abgerundet & & & $\mathrm{Dm}=36 \mathrm{~m}$ \\
\hline & $\mathrm{K}_{2}$ & 24 & 2 & $\mathrm{~S}$ & abgerundet & & & $\mathrm{Dm}=3 \mathrm{Im}$ \\
\hline & $\mathrm{K}_{3}$ & I8 & I & $\mathrm{S}$ & abgerundet & & & $\mathrm{Dm}=24 \mathrm{~m}$ \\
\hline & $\mathrm{K}_{5}$ & 28 & $\mathrm{I}, 5$ & $\mathrm{~S}$ & abgerundet & & & $\mathrm{Dm}=42 \mathrm{~m}$ \\
\hline & $\mathrm{K}_{2 \mathrm{I}}$ & 9 & 0,3 & keine & abgerundet & & & $\mathrm{Dm}=\mathrm{I} 5 \mathrm{~m}$ \\
\hline & $\mathrm{K}_{3} \mathrm{O}$ & I8 & 0,5 & keine & abgerundet & & & $\mathrm{Dm}=24 \mathrm{~m}$ \\
\hline \multirow[t]{3}{*}{ Gf. Boroldaj } & $\mathrm{K} 25$ & 83,5 & 6 & SW & abgerundet & & $\mathrm{x}$ & $\mathrm{x}$ \\
\hline & $\mathrm{K} 27$ & 67,3 & $6, \mathrm{I}$ & S & abgerundet & & & $\mathrm{x}$ \\
\hline & $\mathrm{K} 29$ & 69,3 & 4,6 & $?$ & abgerundet & & & $\mathrm{x}$ \\
\hline Gf. Ereul & $\mathrm{K} 2 \mathrm{O}$ & $5^{\mathrm{I}}$ & 3 & S & abgerundet & & & $\mathrm{Dm}=59 \mathrm{~m}$ \\
\hline Gf. Issyk & K8 & 47 & 5 & SO & abgerundet & & & eventuell \\
\hline \multirow[t]{2}{*}{ Gf. Turgen } & $\mathrm{K}_{3}$ & 70 & 7 & $\mathrm{~S}$ & abgerundet & $\mathrm{x}$ & & \multirow{2}{*}{$\begin{array}{l}\mathrm{Dm}=200 \mathrm{~m} \\
\text { drei Steinkreise } \\
\text { um Kurgan? }\end{array}$} \\
\hline & $\begin{array}{l}\text { „Karakemerskij } \\
\text { Kurgan“ - (I934) } \\
\text { R. Sulejmanov }\end{array}$ & I 48 & 7,5 & $?$ & $?$ & & & \\
\hline \multirow[t]{6}{*}{ Gf. Žalauly-2 } & $\mathrm{K} 2$ & 36 & 3,5 & $\mathrm{~S}$ & abgerundet & & & $\mathrm{Dm}=57 \mathrm{~m}$ \\
\hline & $\mathrm{K}_{3}$ & I5 & 0,5 & S & abgerundet & & & $\mathrm{Dm}=25 \mathrm{~m}$ \\
\hline & Ki4 & 20 & I & S & abgerundet & & & $\mathrm{Dm}=23 \mathrm{~m}$ \\
\hline & Ki5 & 9 & 0,7 & S & abgerundet & & & $\mathrm{Dm}=\mathrm{I} 5 \mathrm{~m}$ \\
\hline & Kig & 43 & 6 & S & abgerundet & & & $\mathrm{Dm}=57 \mathrm{~m}$ \\
\hline & $\mathrm{K}_{5} 6$ & I9 & $\mathrm{I}, 3$ & S & abgerundet & & & $\mathrm{Dm}=24 \mathrm{~m}$ \\
\hline Gf. Žylysaj-1 & K8 & 37 & 4 & $\mathrm{~S}$ & abgerundet & & & $\mathrm{Dm}=5 \mathrm{Im}$ \\
\hline \multirow{10}{*}{$\begin{array}{l}\text { Nekropole des } \\
\text { archäologischen } \\
\text { Komplexes } \\
\text { Sarytau }\end{array}$} & KI4 & IO & 0,3 & keine & abgerundet & & & $\mathrm{Dm}=\mathrm{I} 8 \mathrm{~m}$ \\
\hline & $\mathrm{K}_{70}$ & II & 0,3 & keine & abgerundet & & & $\mathrm{Dm}=\mathrm{I} 4 \mathrm{~m}$ \\
\hline & $\mathrm{K}_{7 \mathrm{I}}$ & I2 & 0,3 & keine & abgerundet & & & $\mathrm{Dm}=\mathrm{I} 6 \mathrm{~m}$ \\
\hline & $\mathrm{K}_{72}$ & 8 & 0,3 & keine & abgerundet & & & $\mathrm{Dm}=\mathrm{IIm}$ \\
\hline & $\mathrm{K}_{73}$ & 7 & 0,3 & keine & abgerundet & & & $\mathrm{Dm}=\mathrm{IO} \mathrm{m}$ \\
\hline & $\mathrm{K}_{74}$ & 8 & 0,3 & keine & abgerundet & & & $\mathrm{Dm}=\mathrm{I} 2 \mathrm{~m}$ \\
\hline & $\mathrm{K}_{76} 6$ & 9 & 0,3 & keine & abgerundet & & & $\mathrm{Dm}=\mathrm{I} 3 \mathrm{~m}$ \\
\hline & $\mathrm{K}_{77}$ & $\mathrm{I} 2$ & 0,3 & keine & abgerundet & & & $\mathrm{Dm}=\mathrm{I} 6 \mathrm{~m}$ \\
\hline & K79 & II & 0,3 & keine & abgerundet & & & $\mathrm{Dm}=\mathrm{I} 5 \mathrm{~m}$ \\
\hline & $\mathrm{K} 82$ & IO & 0,3 & keine & abgerundet & & & $\mathrm{Dm}=\mathrm{I} 3 \mathrm{~m}$ \\
\hline
\end{tabular}




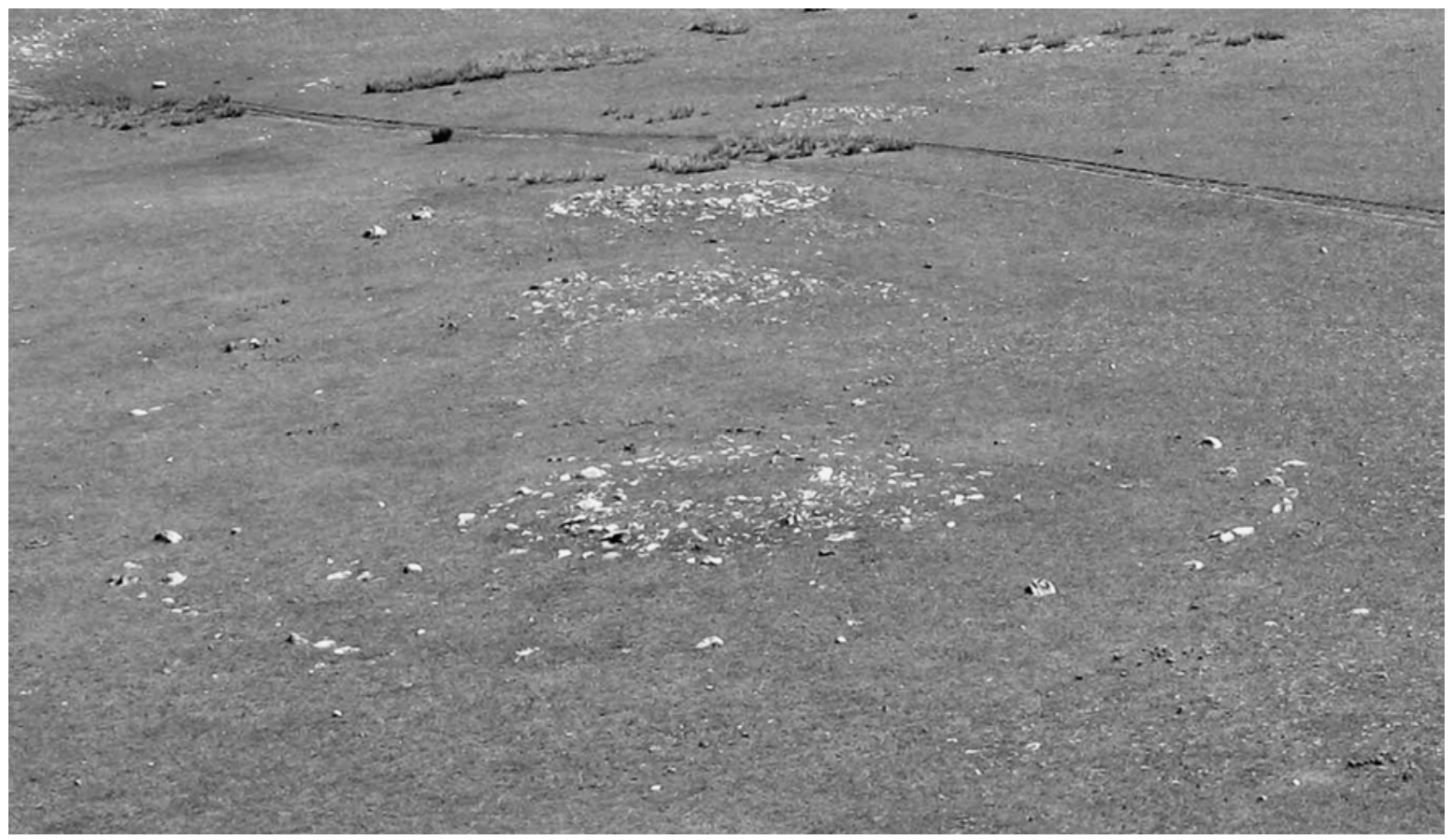

Abb. 75 | Steinkreis um den Kurgan 2I des Gräberfeldes Aktasty-3. Blick von S

Zwölf weitere Kurgane (36\%) zeigten diese Merkmale der Kurganaufschüttung nicht. Bei zwei Kurganen konnte aufgrund des hohen Zerstörungsgrades nicht festgestellt werden, wie die Abhänge angelegt worden waren. Die Kurgane, die einen sanften Abhang aufwiesen, unterschieden sich dadurch voneinander, dass bei I7 Grabhügeln (52\% aller Kurgane mit einem Steinkreis) dieser Abhang exakt nach Süden und jeweils bei einem Kurgan nach Südosten bzw. nach Südwesten ausgerichtet wurde.

Wie die Tabellen I6 und 2I zeigen, traten bei Kurganen mit einem Steinkreis folgende Kombinationen von Konstruktionen in der Peripherie der Kurgane auf: Innerhalb des Steinkreises, der den Kurgan 3 des Gräberfeldes Turgen umgab364, wurden ein sog. Prozessionsweg und 4I kleinere Befunde der Kategorie Peripheriekonstruktionen, nämlich Kleinkurgane festgestellt. Ferner fand sich innerhalb des Steinkreises um den Kurgan 25 der Nekropole Boroldaj365 ein Kreisgraben. Generell wurde festgestellt, dass der Steinkreis immer die äußerste Position innerhalb des Gesamtkomplexes einnahm, falls außer einem Steinkreis noch weitere Konstruktionen am Rande des Kurgans zum Vorschein kamen. Zudem wurden im Grabungsbericht (I934) von R. Sulejmanov in der Beschreibung des „Karakemerskij Kurgans“ vom Gräberfeld Turgen drei Steinkreise um diesen einen Kurgan erwähnt366 (Bernštam/Dublickij I936, 5-6; vgl. Katalog, Gräberfeld Turgen, Forschungsgeschichte).

364 Siehe Katalog, Gf. Turgen, Kurgancharakteristik.

365 Siehe Katalog, Gf. Boroldaj, Kurgancharakteristik.
366 Es könnte jedoch sein, dass man hier eine ähnliche Situation wie beim Kurgan 3 desselben Gräberfeldes hat. Um diesen Kurgan herum wurde ein sog. Prozessionsweg innerhalb eines Steinkreises beobachtet. 
Tabelle 22 | Verhältnis des Durchmessers von Kurganen und sie umlaufenden Steinkreise (nach den Fundorten geordnet)

\begin{tabular}{|c|c|c|c|}
\hline Objekt & Kurgan - Dm (m) & Steinkreis- Dm (m) & $\begin{array}{l}\text { Verhältnis von Kurgan } \\
\varnothing \text { zu Steinkreis }\end{array}$ \\
\hline Gf. Aksaj-9-K4 & $2 \mathrm{I}$ & 32 & 0,66 \\
\hline Gf. Aksaj-9-K7 & 28 & 44 & 0,64 \\
\hline Gf. Aksaj-9 - K1o & 22 & 29 & 0,76 \\
\hline Gf. Aktasty-3 - K1 & 25 & 36 & 0,69 \\
\hline Gf. Aktasty-3 - K2 & 24 & $3 \mathrm{I}$ & 0,77 \\
\hline Gf. Aktasty-3 $-K_{3}$ & I8 & 24 & 0,75 \\
\hline Gf. Aktasty-3 - K5 & 28 & 42 & 0,67 \\
\hline Gf. Aktasty-3-K21 & 9 & I5 & 0,60 \\
\hline Gf. Aktasty-3 - K3o & I8 & 24 & 0,75 \\
\hline Gf. Ereul - K20 & $5 \mathrm{I}$ & 59 & 0,86 \\
\hline Kpx. Sarytau - K14 & IO & I8 & 0,56 \\
\hline Kpx. Sarytau - K7o & II & $\mathrm{I} 4$ & 0,79 \\
\hline Kpx. Sarytau - K71 & $\mathrm{I} 2$ & I6 & 0,75 \\
\hline Kpx. Sarytau - K72 & 8 & II & 0,73 \\
\hline Kpx. Sarytau - K73 & 7 & IO & 0,70 \\
\hline Kpx. Sarytau - K74 & 8 & $\mathrm{I} 2$ & 0,67 \\
\hline Kpx. Sarytau - K76 & 9 & I3 & 0,69 \\
\hline Kpx. Sarytau - K77 & $\mathrm{I} 2$ & 16 & 0,75 \\
\hline Kpx. Sarytau - K79 & II & I5 & 0,73 \\
\hline Kpx. Sarytau - K82 & IO & I3 & 0,77 \\
\hline Gf.Turgen $-K_{3}$ & 70 & 200 & 0,35 \\
\hline Gf. Žalauly-2 - K2 & 36 & 57 & 0,63 \\
\hline Gf. Žalauly-2 - K3 & I5 & 25 & 0,60 \\
\hline Gf. Žalauly-2 - K14 & 20 & 23 & 0,87 \\
\hline Gf. Žalauly-2 - K15 & 9 & I5 & 0,60 \\
\hline Gf. Žalauly-2 - K19 & 43 & 57 & 0,75 \\
\hline Gf. Žalauly-2 - K56 & I9 & 24 & 0,79 \\
\hline Gf. Ž ${ }^{\prime} l y s a j-1-K 8$ & 37 & $5 \mathrm{I}$ & 0,73 \\
\hline
\end{tabular}

Die Steinkreise wurden aus rötlichen 367 bzw. hellgrauen bis weißgrauen 368 , mittelgroßen Steinen $(60-20 \times 40-10 \times 30-5 \mathrm{~cm})$ gebaut. Die hellgrauen bis weißgrauen Flusskieselsteine wurden in einem Kreis angeordnet, die rötlichen Steine meist senkrecht aufgestellt.

367 Z. B.: Gf. Aksaj-9, Kurgane 4 oder 7; siehe Katalog, Gf. Aksaj-9, Kurgancharakteristik.
368 Z. B.: Gf. Turgen, Kurgan 3; siehe Katalog, Gf. Turgen, Kurgancharakteristik. 
Die Steinkreise wiesen einen Durchmesser von I5 m369 bis zu 200 m370 auf. Der Abstand zwischen dem Rand eines Kurgans und dem Steinkreis maß zwischen I,5 m371 und 6I m372.

Das Verhältnis von Kurgandurchmessers zum Durchmesser eines Steinkreises zeigte keine Regelhaftigkeit. Wie die Tabelle 22 zeigt, wurden sogar innerhalb eines Gräberfeldes relativ große Schwankungen festgestellt. Das Verhältnis von Kurgandurchmesser zum Durchmesser des Steinkreises variierte von 2:573 bis zu 4:5774. Die bloße Vermessung der Grabanlage und ihres Umfeldes ließ folgende Fragen: „An welchen Kurganen wurden Steinkreise gebaut?“; „Warum wurden gerade um diese Kurgane herum Steinkreise errichtet?“; „Welche Intention bestimmte die Größe der Steinkreise?“.

Für Steinkreise, die um Großkurgane herum angelegt wurden, lassen sich keine eindeutigen Vergleiche finden. Eine gewisse Ähnlichkeit besteht zu den Steinkreisen der Kleinkurgane auf der IšimEbene Nordkasachstans (Parzinger 2006, 787) und denen im mittleren Dnepr-Gebiet in der Ukraine (Mozolevskij/Polin 2005, 294).

\subsubsection{Sonderfälle}

Auf sechs Nekropolen, die auf alle Verbreitungszonen der früheisenzeitlichen, sakischen Gräberfelder mit großen, fürstlichen Kurganen verteilt sind, wurden insgesamt I3 Kurgane mit verschiedenen, architektonischen Befunden in der Peripherie der Kurgane festgestellt (Tabelle 23; Karte I6). Es handelt sich dabei um Kurgan 6 der Nekropole Asy Saga375, der an der Nordseite eine Art „Vorsprung“ aus Erde und Steinschutt aufwies376, um acht Kurgane des Gräberfeldes Boroldaj377 und Kurgan 3 der Nekropole Ulžan $37^{8}$, die von einem Erdwall umgeben waren. Kurgan 2 des Gräberfeldes auf dem Plateau Kegen war auf einer viereckigen Plattform erbaut worden, deren Seiten an den vier Haupthimmelsrichtungen ausgerichtet worden waren. Von dieser Plattform gehen strahlenförmig angeordnete Stege ab, die als Überreste der Baurampen interpretiert werden können. Ein weiteres Phänomen stellte ein Steinwall dar, der Kurgan 7 der Nekropole Turgen umgab. Der Flachkurgan 8 des Gräberfeldes Žalauly- 8 ist lediglich durch einen doppelten Steinkreis heute noch sichtbar.

Von diesen I3 Kurganen können elf als Groß- (4\% aller untersuchten Großkurgane) und einer als Kleinkurgan (o,2\% aller untersuchten Kleinkurgane) bezeichnet werden. Zudem kam noch ein Flachkurgan zum Vorschein. Die Großkurgane dieser Art sind den zwei größeren Kategorien von Kurgangrößen zuzuordnen. Es handelte sich um neun größere Kurgane (Höhe 4-8 m) und zwei sehr große Kurgane (Höhe über $8 \mathrm{~m}$ ). Die großen Kurgane, deren Höhe zwischen $2 \mathrm{~m}$ und $4 \mathrm{~m}$ maß, wiesen gar keine Sonderfälle in ihrer Peripherie auf. Zehn Kurgane (77\% aller Kurgane mit Sonderfällen in der Peripherie) hatten drei steile und einen sanften Abhang. Zwei weitere Tumuli wiesen diese Merkmale

369 Kleinster Steinkreis - Gf. Žalauly-2, KI5; siehe Tabelle 2I, vgl. Katalog, Gf. Žalauly-2, Kurgancharakteristik.

370 Größter Steinkreis - Gf. Turgen, K3; siehe Tabelle 2I, vgl. Katalog, Gf. Turgen, Kurgancharakteristik.

37 Gf. Žalauly-2, Ki4 und Nekropole des archäologischen Komplexes Sarytau, K70, K72, K73 und K82; siehe Katalog, Gf. Žalauly-2 und Nekropole des Komplexes Sarytau, Kurgancharakteristik.

372 Gf. Turgen, K3; siehe Katalog, Gf. Turgen, Kurgancharakteristik.

373 Gf. Turgen, K3; siehe Tabelle 22, vgl. Katalog, Gf. Turgen, Kurgancharakteristik.
374 Gf. Ereul, K2o und Gf. Žalauly-2, KI4 und K56; siehe Tabelle 22, vgl. Katalog, Gf.r Ereul und Gf. Žalauly-2, Kurgancharakteristik.

375 Siehe Katalog, Gf. Asy Saga, Kurgancharakteristik.

376 Da die Peripherie des Kurgans zum Teil überpflügt wurde, ist nicht auszuschließen, dass es sich hier ursprünglich um eine Plattform gehandelt hat, auf der dieser Kurgan gestanden hat, bevor sie später durch den Pflug zerstört wurde.

377 Kurgane 5, 8-9, I2-I3, 26 und 28; siehe Katalog, Gf. Boroldaj, Kurgancharakteristik.

378 Siehe Katalog, Gf. Ulžan, Kurgancharakteristik. 
Tabelle 23 | Kurgane mit Sonderfällen in ihrer Peripherie

\begin{tabular}{|c|c|c|c|c|c|c|c|}
\hline$\frac{\overrightarrow{0}}{0}$ & $\frac{\frac{\pi}{d}}{\frac{\pi}{0}}$ & 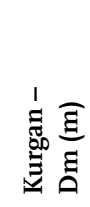 & 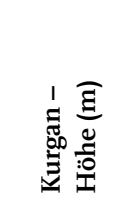 & 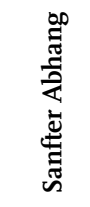 & 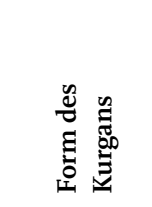 & 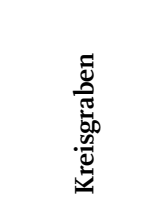 & 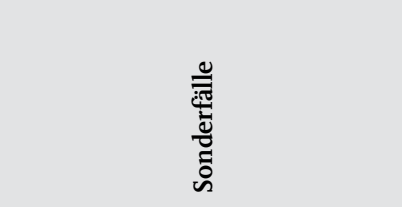 \\
\hline Gf. Asy Saga & K6 & 68 & 5 & SW & abgerundet & & $\begin{array}{l}\text { an der Nordseite ist eine Art } \\
\text { „Vorsprung“ aus Erde und Stein- } \\
\text { schutt gebaut; } \mathrm{B}=22 \mathrm{~m} \text {; } \mathrm{L}=52 \mathrm{~m} \text {; } \\
\mathrm{H}=0,4-0,5 \mathrm{~m}\end{array}$ \\
\hline \multirow[t]{8}{*}{ Gf. Boroldaj } & $\mathrm{K}_{5}$ & 83 & 9,4 & keine & abgerundet & $\begin{array}{l}\mathrm{Dm}=85 \mathrm{~m} ; \\
\mathrm{B} \text { I,om; } \mathrm{T} \text { ?; } \\
\mathrm{W}+\mathrm{SW} \\
\text { zwei Ein- } \\
\text { gangsberei- } \\
\text { che }\end{array}$ & ein Erdwall \\
\hline & K8 & 30 & $\mathrm{I}, 6$ & $\mathrm{~S}$ & abgerundet & ja, B ?; T ? & ein Erdwall \\
\hline & K9 & 37,8 & 4 & SO & abgerundet & ja, B ?; T ? & ein Erdwall \\
\hline & Kio & 44,8 & 5 & SO & abgerundet & $\begin{array}{l}\text { ja, 2x, } \\
\text { Graben- } \\
\text { Wall- } \\
\text { Graben; } \\
\text { B I,5m; } \\
\text { T o,5-I,om }\end{array}$ & ein Erdwall \\
\hline & KI2 & 42,6 & 4,8 & $\mathrm{~S}$ & abgerundet & & ein Erdwall \\
\hline & $\mathrm{K}_{3} 3$ & 43,4 & 4,5 & $\mathrm{~S}$ & abgerundet & & ein Erdwall \\
\hline & $\mathrm{K} 26$ & $5^{\mathrm{I}, 5}$ & 4,7 & W & abgerundet & ja, B ?; T ? & ein Erdwall \\
\hline & $\mathrm{K} 28$ & 46,2 & $?$ & $\mathrm{~S}$ & abgerundet & ja, B ?; T ? & ein Erdwall \\
\hline $\begin{array}{l}\text { Gf. auf dem } \\
\text { Plateau } \\
\text { Kegen }\end{array}$ & $\mathrm{K}_{2}$ & I45 & I3 & $\mathrm{S}$ & abgerundet & & $\begin{array}{l}\text { auf viereckiger Plattform (Seiten- } \\
\text { länge } \mathrm{I} 64 \mathrm{~m} \text {; Durchmesser zu- } \\
\text { sammen mit Baurampen } 330 \mathrm{~m} \text { ), } \\
\text { Plattform an den Haupthimmels- } \\
\text { richtungen orientiert }\end{array}$ \\
\hline Gf. Turgen & $\mathrm{K}_{7}$ & 54 & 5 & $\mathrm{~S}$ & abgerundet & & $\begin{array}{l}\text { ursprünglich ein Steinwall } \\
\mathrm{Dm}=85 \mathrm{~m} ; \mathrm{B}=3 \mathrm{~m} ; \mathrm{H}=0, \mathrm{Im}\end{array}$ \\
\hline Gf. Ulžan & $\mathrm{K}_{3}$ & 36 & 4 & $?$ & abgerundet & & $\begin{array}{l}\text { ein Erdwall, direkt am Kurgan- } \\
\text { rand, } B=4,2 \mathrm{~m} ; \mathrm{H}=2,3 \mathrm{~m}\end{array}$ \\
\hline Gf. Žalauly-8 & K8 & 5 & $\begin{array}{l}\text { Flach- } \\
\text { kurgan }\end{array}$ & & abgerundet & & doppelter Steinkreis \\
\hline
\end{tabular}

nicht auf, und bei einem Kurgan konnten aufgrund des hohen Zerstörungsgrades der Aufschüttung diesbezüglich keine Aussagen getroffen werden. Bei Kurganen mit einem sanften Abhang wurde dieser in sieben Fällen exakt nach Süden (54\% aller Kurgane mit Sonderfällen in der Peripherie), in zwei Fällen nach Südosten (I5\%) und in einem Fall exakt nach Westen ausgerichtet. Alle Kurgane hatten eine abgerundete Form mit abgeflachter Kuppe.

Die häufigsten Konstruktionen in der Peripherie der Kurgane sind Erdwälle. Alle neun Beispiele wurden unweit voneinander entfernt, auf zwei Nekropolen im nordwestlichen Stadtbereich Almatys, festgestellt. Die Entfernung zwischen den beiden Gräberfeldern Boroldaj und Ulžan, auf denen sich einige Kurgane mit Erdwällen befinden, misst ca. 2,0-2,5 km. Die Erdwälle wurden direkt am Kurgan- 
rand errichtet. Bei fünf Kurganen (siehe Tabelle 23) wurde zuerst ein Kreisgraben ausgehoben und mit dem Aushub zugleich ein äußerer Wall aufgeschüttet. Aus diesem Grund bestimmte die Masse des Aushubes das Ausmaß des Walles. Am Kurgan ro derselben Nekropole befand sich der Erdwall ebenso direkt am Rand des Kurgans, allerdings wurde er von zwei Kreisgräben flankiert. So entstand um den Kurgan herum ein komplexes Graben-Erdwall-Graben-System. Am Rande der Kurgane i2 und I3 des Gräberfeldes Boroldaj und um Kurgan 3 der Nekropole Ulžan war nur ein Erdwall angelegt. Hier muss man davon ausgehen, dass das Erdreich für die Wälle nicht aus einem Kreisgraben stammte, sondern andernorts entnommen wurde.

Aus dem skytho-sakischen Kulturkreis sind Analogien für Wälle, die einen Kurgan umgeben, außer aus dem südöstlichen Siebenstromland auch von der Išim-Ebene in Nordkasachstan - Kurgan Kara-Oba (Chabdulina I994, 24, Taf. 30,A) - und aus dem Gebiet am mittleren und unteren Dnepr in der Ukraine bekannt - Aleksandropol'-Kurgan, Kurgane Babina Mogila, Slonovskaja Bliznica, Geremesov, Oguz und Solocha (Aleksandropol'skij kurgan I866, I; Bliznicy Slonovskie I872, 64; Geremesovy kurgany I872, 30; Fialko I994, I38; Mozolevskij/Polin 2005, 107, 298, Taf. 23,I; Boltrik/Fialko 2007, 268-269, Abb. I; Polin/Daragan 2008, I55-156). Weitere Beispiele finden sich um die Kurgane der nördlichen Waldsteppenzone der Ukraine - Kurgan Ostraja Mogila (OAK I889, 29-3I; Mozolevskij/Polin 2005, 298) - sowie im nördlichen Kaukasus - Kurgan 4 beim Dorf Gojty und ein Großkurgan der Nekropole Gorgippija (Markovin I965, I66; Kruglikova I980, 7; Mozolevskij/Polin 2005, 298).

Ein weiterer, allerdings aus Steinen erbauter Wall (siehe Tabelle 23), ist vom Kurgan 7 des Gräberfeldes Turgen bekannt. Der Steinwall befand sich I4-I5 m vom Rand des Kurgans entfernt und hatte eine Breite von $3 \mathrm{~m}$ sowie eine noch messbare Höhe von o,I m. Mit dem Steinwall um den Kurgan belief sich der Gesamtdurchmesser des Ensembles auf $85 \mathrm{~m}$. Momentan sind keine Parallelen für solche Steinwälle bekannt379.

An der Nordseite des Kurgans 6 der Nekropole Asy Saga wurde eine Art „Vorsprung“ festgestellt (siehe Tabelle 23), der aus Erde und Steinschutt gebaut worden ist. An der modernen Oberfläche war er dank einer noch erhaltenen Höhe von $0,4-0,5 \mathrm{~m}$ gut sichtbar. Der „Vorsprung“ hatte ein Ausmaß von $52 \mathrm{~m}$ Länge und $22 \mathrm{~m}$ Breite (vgl. Gass 20IIa, 67, Fig. 22; Gass 20IIb, 2I5, Abb. I4). Die anderen Seiten des Kurgans waren durch den Pflug gestört. Es ist möglich, dass es sich hier um die Überreste einer Plattform handelt, auf der der Kurgan einst stand.

Auf dem Plateau Kegen befand sich der größte Kurgan mit Sonderfällen in der Peripherie. Kurgan 2 des Gräberfeldes war auf einer optisch erfassten viereckigen Plattform mit i $64 \mathrm{~m}$ Seitenlänge und einer noch erhaltenen Höhe von ca. I m erbaut. Die Seiten waren nach den vier Haupthimmelsrichtungen orientiert (siehe Tabelle 23). Der Kurgan hatte einen Durchmesser von I45 m und eine noch erhaltene Höhe von I2 m. Zusammen mit der Plattform erreichte der Tumulus eine Gesamthöhe von I3 m. Vom nördlichen und westlichen Rand gingen strahlenförmig fünf 6-Io $\mathrm{m}$ breite Stege von der Plattform ab (Abb. 76).

Im Sommer 2009 wurden diese Strukturen durch die Kasachisch-Deutsche Archäologische Expedition untersucht380. Es stellte sich heraus, dass es sich hier um die Überreste von Baurampen handelt,

379 Ein Steinwall um den Kurgan I des Gräberfeldes Bajkara im Nordkasachstan soll hier lediglich erwähnt werden. Dieser Steinwall ist als eine der Bauphasen zu verstehen (Parzinger u.a. 2003, 44-48, I02, Abb. 45-50, 80-8I, 84). Im Zuge der weiteren Errichtung der Kurganaufschüttung wurde dieses Element eingebettet. Der Stein- wall von Turgen dagegen blieb als separates Element bestehen.

380 Für die Information bezüglich der Grabungskampagne 2009 im Siebenstromland möchte ich mich bei Prof. Dr. H. Parzinger, Dr. A. Nagler, Dr. M. Nawroth, PD Dr. Z. Samašev und M.-R. Boroffka bedanken. 
Abb. 76 | Gräberfeld auf dem Plateau Kegen. Kurgan 2. 3D-Modell von M. Dörre und Ch. Voll (Beuth-Hochschule für Technik Berlin)

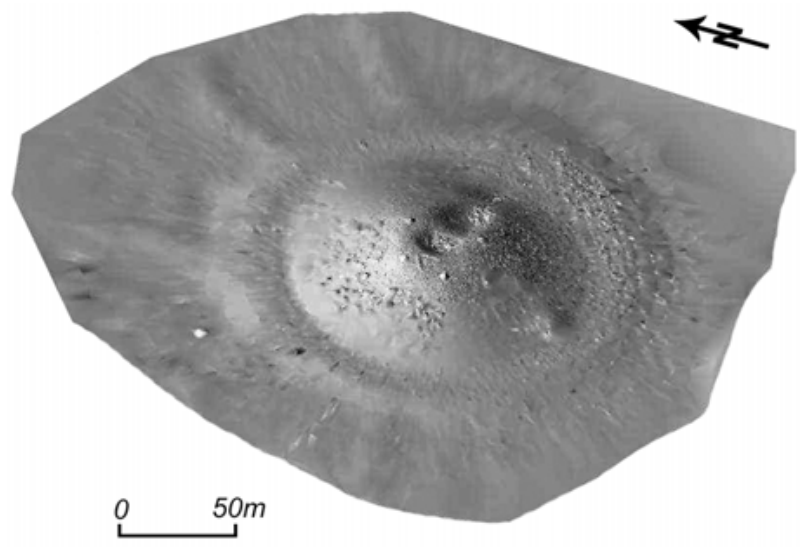

die zur Plattform führten. Insgesamt konnten fünf solche Rampen erfasst werden, die sich nördlich und westlich vom Kurgan befanden. Da die modernen landwirtschaftlichen Aktivitäten besonders die Südhälfte des Kurganumfeldes beschädigt hatten, waren hier die erwähnten Rampen nicht mehr deutlich erkennbar. Ausgehend von der Vermutung, dass die Erbauer des Grabhügels eine symmetrische Struktur schaffen wollten, kann man sich südlich und östlich vom Kurgan weitere Baurampen vorstellen. Insgesamt könnten so um den ganzen Kurgan neun oder zehn solcher Rampen stehen.

Um diese einzigartige radiale Bauweise zu verstehen, wurden im nordwestlichen Peripheriebereich des Kurgans drei Flächen freigelegt (Abb. 77).

Fläche I $(34 \times 27 \mathrm{~m})$ befand sich in einer Absenkung, im rechten Winkel zum Kurgan und schnitt sowohl die Plattform bis zum Kurganrand als auch eine Baurampe an (Abb. 77-78,I.4). Fläche 2 (20,5× $2 \mathrm{~m}$ ) lag quer zu einer Baurampe (Abb. 77-78,2) und die Fläche $3(20,5 \times 2 \mathrm{~m})$ erfasste den Außenrand der Kurganperipherie (Abb. 77-78,3). Die Untersuchungen aller drei Flächen ermöglichten die obengenannten Schlussfolgerungen. Die Stratigraphie der Fläche I unterschied sich von der Stratigraphie der Flächen 2 und 3 (Abb. 79).

Am Kurganrand, südöstliche Hälfte der Fläche I, lag auf dem gewachsenen Boden, der aus einer Sand-Kies-Mischung bestand, eine bis $0,5 \mathrm{~m}$ mächtige Lehmschicht, die im nördlichen Verlauf stetig nachließ. Der Lehm wurde mit einer bis zu o,3 $\mathrm{m}$ starken Mischschicht aus Humus und Lehm bedeckt. In der Nordecke der Fläche befand sich direkt unter dieser Schicht die Kulturschicht, die sich als eine bis zu o,2 m starke, dunkelgraue, feinsandige Schicht mit Holzkohlenpartickeln, Tierknochen ${ }^{88}$ und wenigen handgefertigten, eisenzeitlichen Scherben 382 erwies. Die wenigen hier geborgenen Funde ließen keine eindeutigen Schlussfolgerungen bezüglich der sakralen oder profanen Bedeutung dieser Schicht zu. Jedoch wurde aus den Tierknochen das Material für die ${ }^{14 C}$-Analyse gewonnen, die diese Schicht in das 5. Jh. v. Chr. datieren ließ383. Ebenso lag in der Nordecke der Fläche zwischen der Kulturschicht und dem gewachsenen Boden eine dünne, max. bis zu o,2 m Lehmschicht mit höherem Sandund Kiesanteil.

38I Zur archäozoologischen Bestimmung der Fläche I der Peripherie des Kurgans 2 in der Nekropole auf dem Plateau Kegen siehe Katalog, Gf. auf dem Plateau Kegen, Kurgancharakteristick, Kurgan 2.

382 Zur Keramik der Fläche I der Peripherie des Kurgans 2 von der Nekropole auf dem Plateau Kegen siehe Katalog,
Gf. auf dem Plateau Kegen, Kurgancharakteristik, Kurgan 2.

383 Genaue Nachweise zur Datierung siehe im Katalog, Gf. auf dem Plateau Kegen, Kurgan 2, Kurgancharakteristik sowie im Appendix I Nr. I3. 


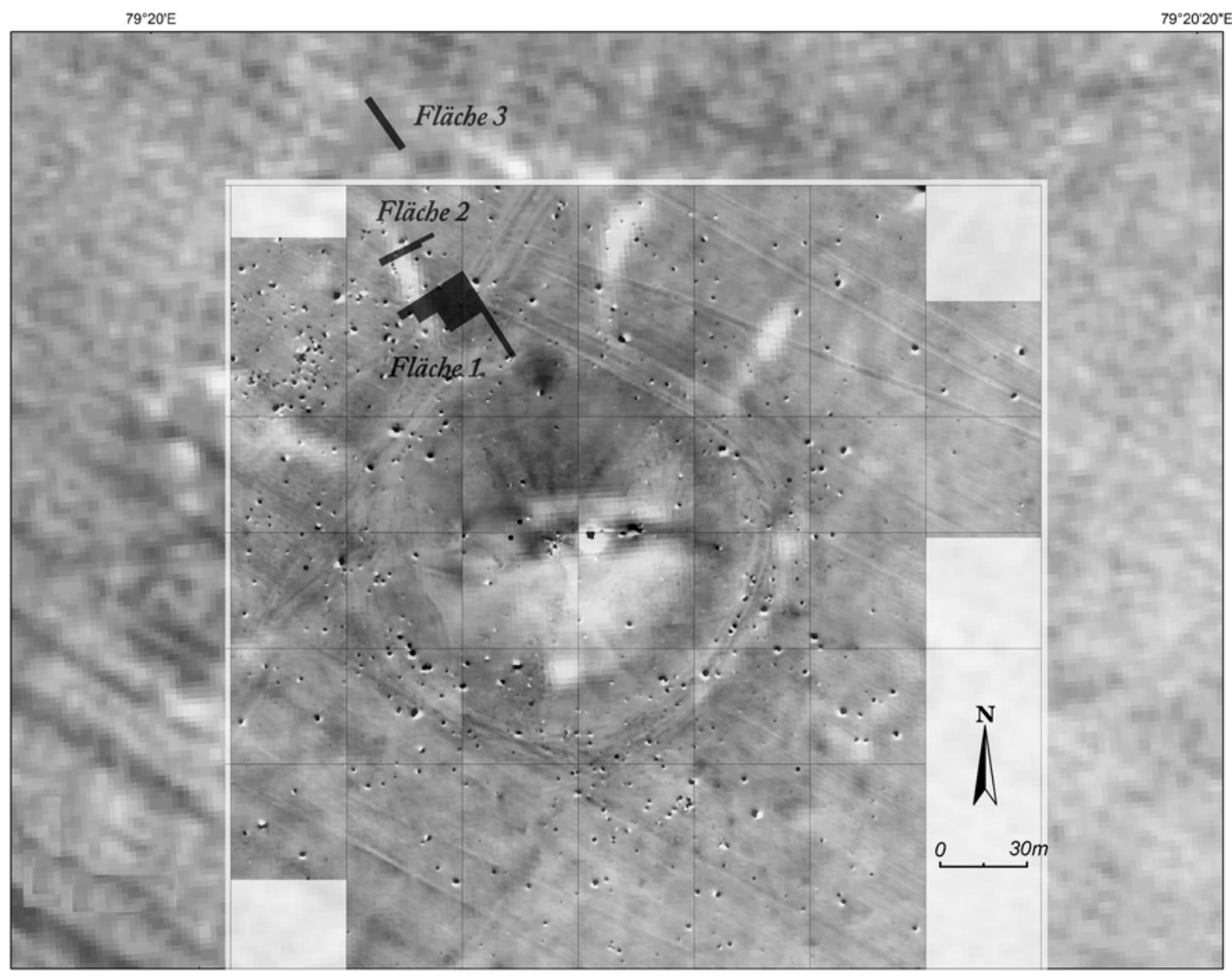

Abb. 77 | Gräberfeld auf dem Plateau Kegen. Kurgan 2. Die Lokalisation der Grabungsflächen der Kasachisch-Deutschen Archäologischen Expedition 2009. Kombination der SPOT Aufnahme 2,5 $\mathrm{m}$ und der geomagnetischen Prospektion von J. Faßbinder und T. Gorka - BLfD, München

Die stratigraphische Situation der Fläche 2 ähnelte der Fläche 3 (Abb. 79). Hier lag auf dem gewachsen Boden eine $0,25-0,7 \mathrm{~m}$ mächtige Lehmschicht, die mit einer Humus-Lehm-Mischschicht überdeckt wurde. Auf den Flächen 2 und 3 wurden keine weiteren Funde oder Befunde festgestellt.

Die untersuchten Flächen, vor allem Fläche I mit dem Profil I, zeigten, dass für den Großkurgan keine eigene Plattform errichtet werden musste: Die Träger der sakischen Kultur hatten eine natürliche Erhebung für die Baufläche ausgesucht und durch das Ausheben der Segmente an den Abhängen dieser Erhebung eine Plattform erzeugt, wobei die stehen gelassenen Stege als Baurampen dienten.

Mit den Baurampen besaß die Konstruktion einen Gesamtdurchmesser von 330 m. Die Erde aus dem Bereich zwischen den Rampen wurde als Baumaterial für die Kurganaufschüttung verwendet. Die Baurampen wurden nach dem Bau des Kurgans nicht beseitigt, so dass sie als zusätzliche architektonische Elemente der Anlage angesprochen werden können, die ihr noch mehr Monumentalität verlieh.

Während solche Plattformen, die unter früheisenzeitlichen Fürstenkurganen errichtet wurden, außer aus dem südöstlichen Siebenstromland noch aus Ostkasachstan bekannt sind (Kurgane des Gräber- 


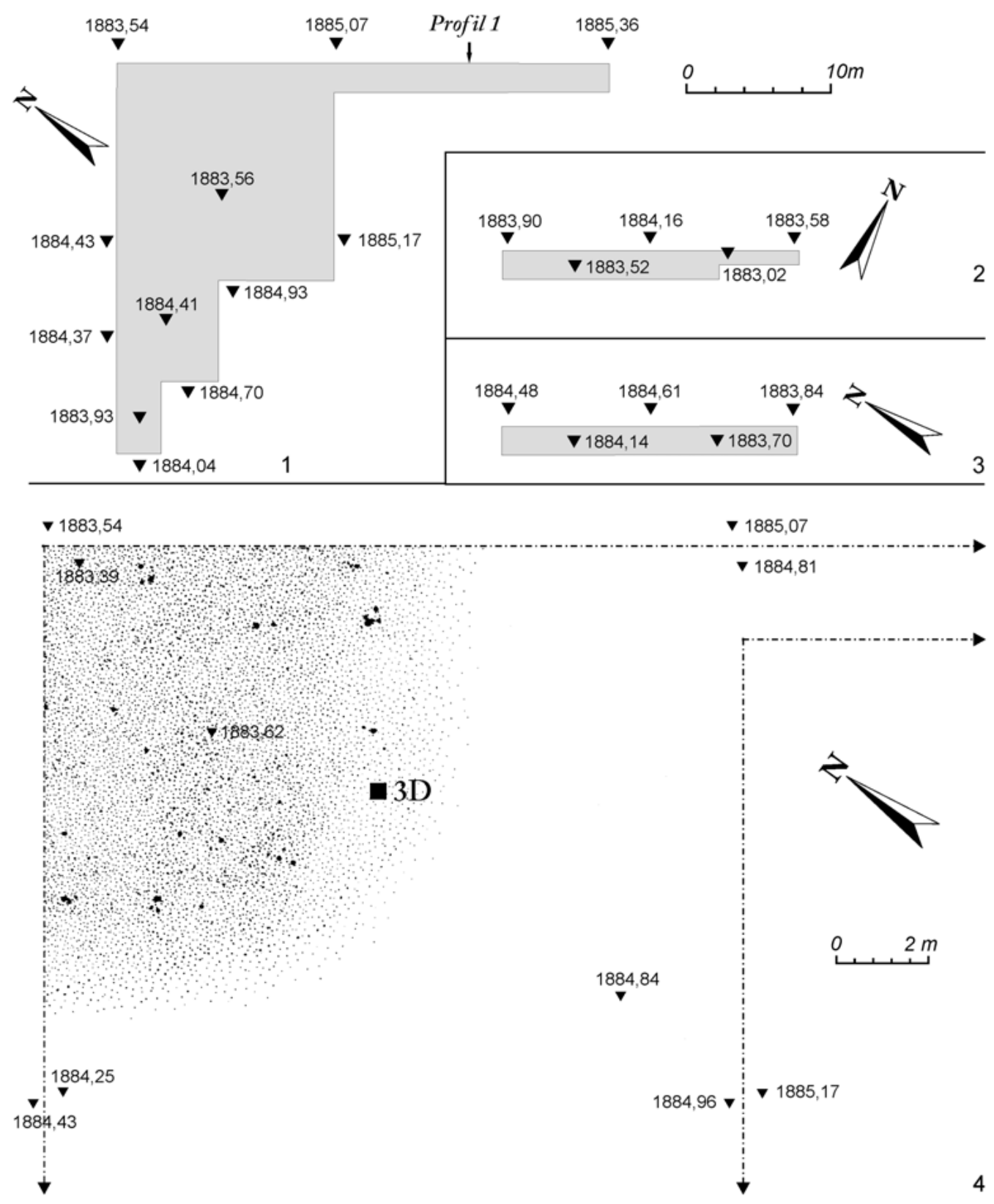

Abb. 78 | Gräberfeld auf dem Plateau Kegen. Kurgan 2. Schematische Darstellung der Untersuchungsflächen (I. Fläche I; 2. Fläche 2; 3. Fläche 3; 4. Fragment des Planums von der Fläche I mit der Kulturschicht)

feldes Aksuat - Nagler 2010, 387-388; Nagler/Samašev 20Io, 47-48; Samašev u.a. 2010, 94-95), stellen die Baurampen in der Peripherie des Kurgans 2 der Nekropole auf dem Plateau Kegen für diesen Kulturraum zunächst eine einmalige Besonderheit dar.

Der Kurgan 8 der Nekropole Žalauly- 8 erwies sich als ein Flachkurgan, den ein noch sichtbarer, doppelter Steinkreis markierte (siehe Tabelle 23). Mit einem Durchmesser von nur $5 \mathrm{~m}$ kann er als Kleinkurgan angesprochen werden. Der Abstand zwischen den Steinkreisen betrug I $\mathrm{m}$. 


\section{$1 \square 2 \square 3 \square 4 \square+5$}

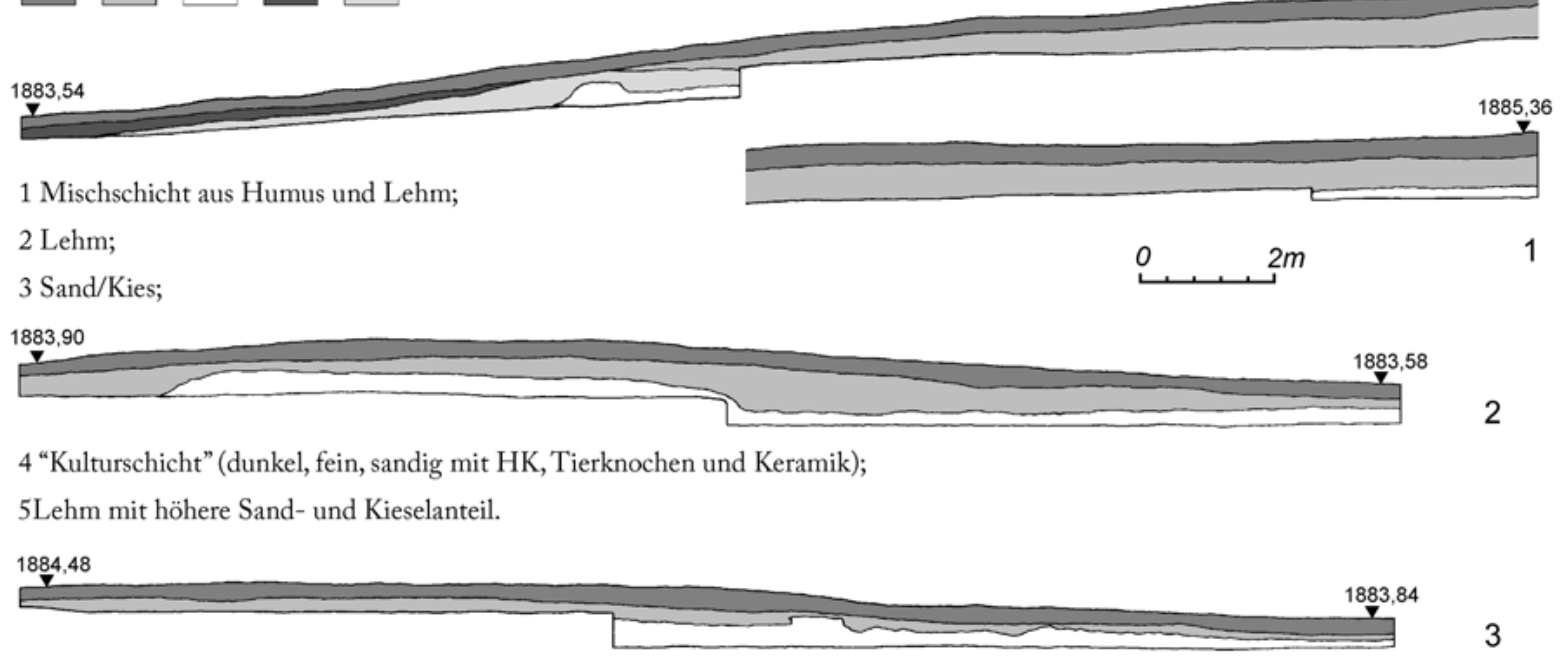

Abb. 79 | Gräberfeld auf dem Plateau Kegen. Kurgan 2. Profile der untersuchten Flächen (ז. Profil I /NW-SO/ der Fläche I; 2. Profil I /SW-NO/ der Fläche 2; 3. Profil I /NW-SO/ der Fläche 3)

\subsubsection{Viereckige Kurgane}

I6 Kurgane384 von zehn untersuchten Gräberfeldern (Tabelle 24; Karte I7) wiesen statt einer abgerundeten Form mit abgeflachter Kuppe eine viereckige pyramidenähnliche Form mit einer abgeflachten Kuppe auf (Abb. 28).

Bisher waren solche viereckigen Konstruktionen innerhalb des früheisenzeitlichen skytho-sakischen Kulturkreises nur für die Tagar-Kultur Südsibiriens belegt (Paul's 200I, I26; vgl. Mozolevskij/Polin 2005, 295). Ein weiteres Beispiel stellt ein viereckiger Graben um den Kurgan 22 beim Dorf Volčansk im Gebiet nördlich des Asowschen Meeres dar. Da in diesem Fall die Kurganaufschüttung sehr stark vom Pflug beschädigt worden ist, konnten keine Aussagen bezüglich der ursprünglichen Form des Kurgans gemacht werden (Polin/ Kubyšev I997, I9, Abb. I6; Mozolevskij/Polin 2005, 295).

Die Kurgane des südöstlichen Siebenstromlandes mit einer viereckigen Form der Aufschüttung zeigen, dass Grabhügel dieser Form nicht nur in Südsibirien, sondern auch in davon westlich gelegenen Gebieten während der Früheisenzeit vereinzelt im Bereich des skytho-sakischen Kulturkreises vorkommen. Offensichtlich hatte die äußere Form der Kurgane eine bestimmte Bedeutung, die uns jedoch beim gegenwärtigen Kenntnisstand verschlossen bleibt.

Viereckige Kurgane traten in allen Verbreitungszonen auf. Von I6 Kurganen konnten zwölf als Groß- (4\% aller untersuchten Großkurgane) und fünf als Kleinkurgane (0,8\% aller untersuchten Kleinkurgane) bezeichnet werden. Viereckige Großkurgane sind für die zwei kleineren Kategorien von Kurgangrößen belegt. Man fand fünf große $(2-4 \mathrm{~m})^{385}$ und sechs größere Kurgane $(4-8 \mathrm{~m})^{386}$. Sehr

$3842 \%$ aller untersuchten Kurgane im südöstlichen Siebenstromland.
$38531 \%$ aller viereckigen Kurgane.

$38638 \%$ aller viereckigen Kurgane. 
Tabelle 24 | Viereckige Kurgane

\begin{tabular}{|c|c|c|c|c|c|c|c|c|}
\hline 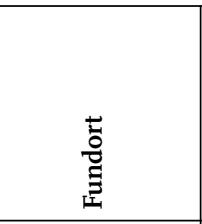 & $\frac{\pi}{\frac{\pi}{2}}$ & 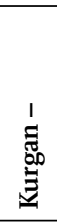 & 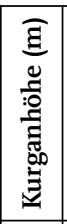 & 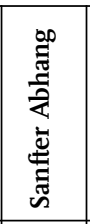 & 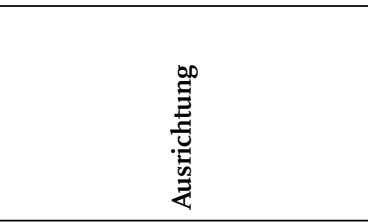 & 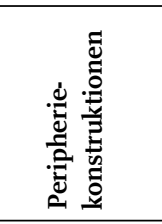 & 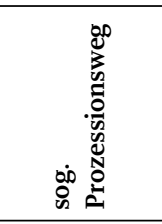 & 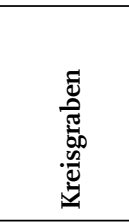 \\
\hline $\begin{array}{l}\text { Ein Kurgan } \\
5 \mathrm{~km} \text { südwest- } \\
\text { lich vom Dorf } \\
\text { Žalauly }\end{array}$ & $\mathrm{IK}_{5} \mathrm{SW}$ & 28 & 3 & keine & $\begin{array}{l}\text { Seiten an den vier Haupt- } \\
\text { himmelsrichtungen orientiert }\end{array}$ & $\begin{array}{l}\text { Kleinkurgan } \\
\text { innerhalb } \\
\text { des Kreis- } \\
\text { grabens }\end{array}$ & & $\begin{array}{l}\text { Dm=73m; } \\
\text { B=Iom; } \\
\mathrm{T}=\mathrm{Im} ; \\
\text { W-Hälfte } \\
\text { Eingangs- } \\
\text { bereich }\end{array}$ \\
\hline Gf. Asy Saga & $\mathrm{K}_{3}$ & 56 & 4 & S & $\begin{array}{l}\text { Seiten an den vier Haupt- } \\
\text { himmelsrichtungen orientiert }\end{array}$ & & $\begin{array}{l}\text { Seitenlänge: } \\
95 \mathrm{~m} ; \\
\mathrm{B}=2,5 \mathrm{~m}\end{array}$ & \\
\hline $\begin{array}{l}\text { Gf. am } \\
\text { Bartogaj-Stausee }\end{array}$ & $\begin{array}{l}\text { Nordost- } \\
\text { ufer - K6 }\end{array}$ & 20 & 0,7 & keine & $\begin{array}{l}\text { Seiten an den vier Haupt- } \\
\text { himmelsrichtungen orientiert }\end{array}$ & & $\begin{array}{l}\text { Seitenlänge } \\
38,5 \mathrm{~m} ; \\
\mathrm{B}=\mathrm{I}, \mathrm{O}-\mathrm{I}, 5 \mathrm{~m}\end{array}$ & \\
\hline Gf. Bes Tobe & $\mathrm{K} 2$ & 33 & 3 & S & $\begin{array}{l}\text { Seiten an den vier Haupt- } \\
\text { himmelsrichtungen orientiert }\end{array}$ & & & \\
\hline $\begin{array}{l}\text { Gf. } \\
\text { Kyzplšaryk }\end{array}$ & $\mathrm{K}_{2}$ & 36 & 4,5 & keine & $\begin{array}{l}\text { Seiten an den vier Haupt- } \\
\text { himmelsrichtungen orientiert }\end{array}$ & & & \\
\hline \multirow[t]{5}{*}{$\begin{array}{l}\text { Gf. } \\
\text { Novoalekseevka }\end{array}$} & $\mathrm{K}_{4}$ & 68 & 6 & keine & $\begin{array}{l}\text { Seiten an den vier Haupt- } \\
\text { himmelsrichtungen orientiert }\end{array}$ & & & \\
\hline & Kio & 67 & 6 & keine & $\begin{array}{l}\text { Seiten an den vier Haupt- } \\
\text { himmelsrichtungen orientiert }\end{array}$ & & & \\
\hline & $\begin{array}{l}\text { „kleines Gräber- } \\
\text { feld“ - K №2 }\end{array}$ & I7 & 0,6 & keine & $\begin{array}{l}\text { Seiten an den vier Haupt- } \\
\text { himmelsrichtungen orientiert }\end{array}$ & & & \\
\hline & $\begin{array}{l}\text { „kleines Gräber- } \\
\text { feld“ - K №3 }\end{array}$ & $?$ & $?$ & keine & $\begin{array}{l}\text { Seiten an den vier Haupt- } \\
\text { himmelsrichtungen orientiert }\end{array}$ & & & \\
\hline & $\begin{array}{l}\text { „kleines Gräber- } \\
\text { feld“ - K №7 }\end{array}$ & IO & 0,2 & keine & $\begin{array}{l}\text { Seiten an den vier Haupt- } \\
\text { himmelsrichtungen orientiert }\end{array}$ & & & \\
\hline Gf. Taučilik-6 & KI & 43 & 4 & S & $\begin{array}{l}\text { Seiten an den vier Haupt- } \\
\text { himmelsrichtungen orientiert }\end{array}$ & & & \\
\hline Gf. Turgen & Kı2 & 42 & 3,5 & S & $\begin{array}{l}\text { Seiten an den vier Haupt- } \\
\text { himmelsrichtungen orientiert }\end{array}$ & & & \\
\hline \multirow[t]{3}{*}{ Gf. Žoan Tobe } & $\mathrm{K} 2$ & 60 & 5,7 & S & $\begin{array}{l}\text { Seiten an den vier Haupt- } \\
\text { himmelsrichtungen orientiert }\end{array}$ & & & \\
\hline & K6 & 52 & 3,7 & S & $\begin{array}{l}\text { Seiten an den vier Haupt- } \\
\text { himmelsrichtungen orientiert }\end{array}$ & & & \\
\hline & K8 & $2 \mathrm{I}$ & $I, 2$ & S & $\begin{array}{l}\text { Seiten an den vier Haupt- } \\
\text { himmelsrichtungen orientiert }\end{array}$ & & & \\
\hline Gf. Žylysaj-1 & K6 & 28 & 3 & S & $\begin{array}{l}\text { Seiten an den vier Haupt- } \\
\text { himmelsrichtungen orientiert }\end{array}$ & & $\begin{array}{l}\text { Seitenlänge: } \\
45 \mathrm{~m} ; \mathrm{B}=\mathrm{Im}\end{array}$ & \\
\hline
\end{tabular}


große Kurgane (von mehr als 8 m Höhe) mit viereckiger, pyramidenähnlicher Form wurden bisher nicht festgestellt.

Das Ausmaß der Kurgane schwankte zwischen o,2 $\mathrm{m}^{387}$ und 6,0 m ${ }^{388}$ Höhe und von Io $\mathrm{m}^{389}$ bis 68 m390 Seitenlänge.

Die Hälfte aller viereckigen Kurgane wies trotz der pyramidenartigen Form drei steile und einen sanften Abhang auf, der in allen Fällen nach Süden ausgerichtet war. Die anderen Kurgane dieser Kategorie hatten gleichmäßig geneigte Abhänge.

Die pyramidenförmigen Kurgane wurden so errichtet, dass ihre Seiten grob an den vier Haupthimmelsrichtungen orientiert waren.

Drei viereckige Kurgane waren von sog. Prozessionswegen umgeben (Tabelle 24), die sich in ihrem Verlauf der viereckigen Form anpassten.

Um einen viereckigen Kurgan, 5 km südwestlich vom Dorf Žalauly (Tabelle 24), wurde ein Kreisgraben mit einem Eingangsbereich an der Westseite ausgegraben. Im Bereich zwischen dem Kurganrand und dem Graben, südsüdöstlich des Kurgans, wurde ein Kleinkurgan festgestellt, der zur Kategorie der Peripheriekonstruktionen gehörte.

Die Kurgane № 2 und № 7 des „kleinen Gräberfeldes“ der Nekropole Novoalekseevka (Kopylov I956, 3-7, I2-2I, Taf. I, 5; Kopylov I958; Archeologičeskaja karta Kazachstana I960, 308, Nr. 4274) und der Kurgan 8 des Gräberfeldes Žoan Tobe (Nagler 2009, 406, Abb. 74-75; Nagler u.a. 20ıo, 50; Samašev u.a. 2009, 351-352) wurden ausgegraben39. Trotz einer Untersuchung der Kurgane konnten keine Unterschiede zu denen mit einer abgerundeten Form der Kurganaufschüttung festgestellt werden. Die Frage nach der Ursache für die ungewöhnliche Form bleibt zunächst offen.

Zusammenfassend lässt sich sagen, dass in zwei Verbreitungszonen des südöstlichen Siebenstromlandes Konzentrationen von Gräberfeldern mit sakischen, fürstlichen Großkurganen vorkommen. Die untersuchten Großkurgane lassen sich in drei Größenkategorien unterteilen. Tumuli der Kategorie „sehr große Kurgane“ (Höhe über $8 \mathrm{~m}$ ) treten in beiden Zonen nicht besonders häufig auf. Grabhügel der Kategorien "größere Kurgane“ (4-8 m) und „große Kurgane“ (2-4 m) sind im nördlich des Transili-Alatau gelegenen Flachland (erste Verbreitungszone) mit fast gleicher Zahl vertreten. Dafür treten große Kurgane (2-4 $\mathrm{m}$ ) besonders häufig in der zweiten Verbreitungszone auf (Hochplateau Kegen sowie im Gebiet nördlich des Kuluktau-Gebirges). Die Analyse des Verhältnisses von Höhe zu Kurgandurchmesser in den Nekropolen beider Verbreitungszonen zeigt, dass sich die Anlagen in dieser Hinsicht nicht unterscheiden. Ebenso ist fast kein Unterschied in beiden Verbreitungszonen im Bezug auf den Anteil von Groß- und Kleinkurganen innerhalb einer Nekropole festzustellen. Dies gilt jedoch nicht für die Anteiligkeit von unterschiedlichen Größenkategorien der Großkurgane innerhalb eines Gräberfeldes. Das nördlich des Transili-Alatau gelegene Flachland wies im Verhältnis mehr höhere Kurgane auf als

387 Gf. Novoalekseevka „kleines Gräberfeld“, K № 7; siehe Tabelle 24, vgl. Katalog, Gf. Novoalekseevka, Forschungsgeschichte.

388 Gf. Novoalekseevka, K4 und KIo, siehe Tabelle 24, vgl. Katalog, Gf. Novoalekseevka, Kurgancharakteristik.

389 Gf. Novoalekseevka „kleines Gräberfeld“, K № 7, siehe Tabelle 24, vgl. Katalog, Gf. Novoalekseevka, Forschungsgeschichte.

390 Gf. Novoalekseevka, K4, siehe Tabelle 24, vgl. Katalog, Gf. Novoalekseevka, Kurgancharakteristik.
39I Das Gf. Novoalekseevka wurde im Jahre I955 zum Teil im Zuge einer Lehrgrabung der Staatlichen Pädagogischen Hochschule „Abaj“ (Almaty) unter der Leitung von I. I. Kopylov untersucht. Die Nekropole Žoan Tobe wurde im Sommer 2008 durch eine Kasachisch-Deutsche Archäologischen Expedition unter der Leitung von H. Parzinger, Z. Samašev, A. Nagler und M. Nawroth zum Teil untersucht. 
das Plateau Kegen und seine Umgebung. Dies betrifft sowohl die Kategorie „sehr große Kurgane“ (mit einer Höhe von über $8 \mathrm{~m}$ ) als auch "größere (4-8 m) und große“ (2-4 m) Kurgane, falls sie mehr als die Hälfte aller Großkurgane eines Gräberfeldes ausmachten.

Es bleibt folglich festzuhalten, dass aufgrund der relativ gleichen Verteilung aller Größenkategorien von Kurganen sowie ganz ähnlicher Größenverhältnisse innerhalb beider Verbreitungszonen ein vergleichbares Bild für beide Zonen entsteht. In der ersten Verbreitungszone jedoch ist der Anteil der größeren Anlagen einer Nekropole wesentlich höher. Folgt man der These, dass die Größe der Kurgane das gesellschaftliche Ansehen der Bestatteten wiederspiegelt (siehe oben; vgl. Akišev/Kušaev i963, 86; vgl. Grač I975, I58-I74; vgl. Akišev K.A. I978, 56; vgl. Mozolevs'kij I979, I56; vgl. Mozolevskij/Polin 2005, 299), kommt man zu dem Schluss, dass im nördlich des Transili-Alatau gelegenen Flachland die Großkurgane auf den Nekropolen eher für die obere Schicht der Elite der bestattenden Gemeinschaft gebaut worden sind. Freilich sind beide Verbreitungszonen für die früheisenzeitlichen Nomadenvölker des südöstlichen Siebenstromlandes von gleich großer Bedeutung gewesen. Davon zeugt die räumliche Dichte der Gräberfelder sowie das Vorkommen sehr großer Kurgane in beiden Zonen („Kurgane-Giganten“ im Sinne Mozolevskijs und Polins: Mozolevskij/Polin 2005, 299).

Über die Hälfte aller Großkurgane wies drei steile und einen sanften Abhang auf. Damit erhielten solche Kurgane zumindest äußerlich Ähnlichkeit mit den skythischen Ares-Tempeln, wie sie von Herodot (Herodot [4-62], 30I-302) beschrieben wurden. Der sanfte Abhang wurde meistens nach Süden ausgerichtet.

Es gibt kein einziges sakisches Gräberfeld mit Großkurganen, das vollständig ausgegraben ist. Einige wenige erforschte Großkurgane des südöstlichen Siebenstromlandes lassen nur durch den Vergleich mit anderen Fürstenkurganen des skytho-sakischen Kulturkreises einige Aspekte zur Kultur der sakischen Elite zu. Die Kleinkurgane dieser Nekropolen, die für das „einfache“ Volk errichtet worden sind, fanden noch weniger Aufmerksamkeit bei den Archäologen. Die geringen Kenntnisse über die Kleinkurgane erlauben momentan nicht, detaillierte Aussagen über die darin Bestatteten zu treffen. Aus diesen Gründen fällt es bislang schwer, Information zur gesamten sozialen Struktur der Saken anhand der Gräber zu erhalten392, da sich die wenigen Untersuchungen bisher fast ausschließlich auf die Großkurgane konzentrierten.

Die Peripherie vieler Kurgane wurde durch moderne Landwirtschaft entweder komplett zerstört oder stark beschädigt. Dem fielen wahrscheinlich gerade solche Konstruktionen zum Opfer, die sich an den Rändern der Grabhügel befanden. Es handelte sich dabei um sog. Prozessionswege, Kreisgräben, Steinkreise, Sonderfälle in der Peripherie der Kurgane oder um die Peripheriekonstruktionen, die in allen Verbreitungszonen der untersuchten, früheisenzeitlichen Gräberfelder mit großen fürstlichen Kurganen der Saken zu finden sind. Kleinkurgane stellen die häufigsten Peripheriekonstruktionen dar. Sie wurden südlich oder östlich vom Großkurgan in einem Abstand von bis zu 37 m Entfernung vom Kurganrand bzw. direkt am Rand des Grabhügels errichtet. Die Anlage von sog. Prozessionswegen ist dagegen nur für das südöstliche Siebenstromland belegt393. Breite und Durchmesser der Wege variieren. Das Verhältnis von Kurgandurchmesser zum Durchmesser des sog. Prozessionsweges bleibt jedoch immer konstant, nämlich 3:5. Eine solche Konstante konnte für das Verhältnis von Kurgandurchmesser zu Kreisgraben nicht festgestellt werden. Dieser Fakt konnte jedoch lediglich innerhalb eines Gräberfeldes beobachtet werden. Von Nekropole zu Nekropole bestehen diesbezüglich jedoch Unterschiede.

392 Eine ähnliche Meinung bezüglich des Forschungsstandes zu den Kurganen der Mozdok-Steppe in Nordkaukasien vertritt auch A. Nagler (Nagler I996, 66).
393 Die „Korridore“ um einige Kurgane im Bergaltai weisen evtl. gewisse Parallelen zu den rituellen Handlungen, jedoch nicht direkt zu der Bautradition auf. 
Solch ein mehr oder weniger konstantes Verhältnis von Kurgandurchmesser zum Durchmesser eines Steinkreises konnte in keinem Fall festgestellt werden. Offensichtlich galten beim Bau solcher Konstruktionen in der Peripherie eines Kurgans andere Regeln. Steinkreise wurden in der Regel als äußerster Bestandteil der Grabanlage errichtet. Zudem traten sie nie im Zusammenhang mit sehr großen Kurganen (Höhe über $8 \mathrm{~m}$ ) auf. Weitere Konstruktionen in der Peripherie der Kurgane sind vor allem Erdund Steinwälle sowie Plattformen und noch erhaltene Rampen, die vom Bau des Grabhügels zeugen. Obwohl solche Konstruktionen in beiden Verbreitungszonen vorkommen, muss ein Unterschied hervorgehoben werden: Die Wälle, die sowohl aus Erde als auch aus Steinen gebaut wurden, sind ausschließlich in der ersten Verbreitungszone, entlang der Nordseite des Transili-Alatau, anzutreffen. Plattformen, auf denen Kurgane errichtet worden sind (vorbehaltlich der Tatsache, dass es sich bei dem „Vorsprung“ am Kurgan 6 des Gräberfeldes Asy Saga tatsächlich um den Überrest einer solchen Plattform handelt), kamen dagegen in beiden Verbreitungszonen zum Vorschein.

Vergleicht man die Peripherie der sakischen Kurgane des südöstlichen Siebenstromlandes mit der Peripherie zeitgleicher Kurgane in anderen Gebieten des skytho-sakischen Kulturkreises, ist festzustellen, dass sog. Prozessionswege, Steinwälle und Baurampen heute nur im südöstlichen Siebenstromland vorkommen.

Das Begrenzen der Peripherie der Kurgane durch sog. Prozessionswege, Kreisgräben, Steinkreise oder Wälle sollte die Welt der Lebenden und der Toten voneinander trennen. Es ist vorstellbar, dass die sakralen Räume, die durch diese Konstruktionen entstanden, tabuisiert waren oder durch den so entstandenen rituellen Kreis die Lebenden vor den Toten beschützen sollten (Rapport/Trudnovskaja I979, I56; Grač I980, 70; Belov/Ljaško I99I, 29; Mozolevskij/Polin 2005, 294). Mit hoher Wahrscheinlichkeit können solche Konstruktionen in der Peripherie der Kurgane des skytho-sakischen Kulturkreises als eine Begrenzung des sakralen Raumes um den Kurgan herum angesprochen werden.

Eine bestimmte, momentan nicht greifbare Bedeutung haben die viereckigen, pyramidenförmigen Kurgane mit abgeflachter Kuppe, die im gesamten südöstlichen Siebenstromland auftreten. Solche Kurgane kommen in den Kategorien Groß- und Kleinkurgane vor. Sehr große Kurgane mit einer pyramidenähnlichen Form, deren Höhe $8 \mathrm{~m}$ übersteigt, wurden allerdings bisher nicht festgestellt. Die Kenntnis von pyramidenförmigen Kurganen im Untersuchungsgebiet verändert zwar das Bild, dass viereckige Grabhügel der frühen Eisenzeit im skytho-sakischen Kulturkreis nur in Südsibirien zu finden sind, allerdings erlaubt der mangelnde Forschungsstand394 nicht, weitere Schlüsse zu ziehen. Auf diese Weise bleiben viele Fragen zur Bedeutung der pyramidenförmigen Kurgane des südöstlichen Siebenstromlandes offen.

\subsection{Die räumliche Struktur der Nekropolen}

Fast alle Kurgane der untersuchten Gräberfelder bilden Kurganketten. Da der Kurgan 5 km südwestlich vom Dorf Žalauly einzeln steht und das Gräberfeld am Bartogaj-Stausee sowie die Nekropole des archäologischen Komplexes Sarytau nur aus Kleinkurganen bestehen, werden sie in diesem Rahmen

394 Bis heute gibt es nur drei viereckige Kurgane, die untersucht wurden (K № 2 und K № 7 des „kleinen Gräberfeldes" der Nekropole Novoalekseevka und K8 des Gf. Žoan Tobe). Alle diese Kurgane sind Kleinkurgane. Zudem wiesen Kurgan № 2 des „kleinen Gräberfeldes“ der
Nekropole Novoalekseevka und Kurgan 8 des Gf. Žoan Tobe durch die Beraubung starke Spuren von Zerstörung auf. In Kurgan № 7 des „kleinen Gräberfeldes“ der Nekropole Novoalekseevka fand man kein Grab. Es handelt sich wohl um einen Kenotaph. 
nicht in die Analyse einbezogen. Bei den folgenden Auswertungen wurden nur diejenigen früheisenzeitlichen Kurgane betrachtet, deren Lage innerhalb eines Gräberfeldes bekannt war395.

\subsubsection{Kettenmuster}

Wie bereits erwähnt wurde, bilden fast alle Kurgane der erforschten Nekropolen Ketten (730 Grabhügeln; $98 \%$ aller untersuchten Fälle), die aus zwei bis 38 Kurganen bestehen und nach unterschiedlichen Himmelsrichtungen ausgerichtet wurden. Ein Gräberfeld kann aus einer oder bis zu elf Ketten bestehen. Falls die Kurganketten Bezug aufeinander nehmen und auf einem bestimmten Raum konzentriert waren, werden sie als zu einem Gräberfeld zugehörig angesprochen.

Das Ausmaß des Gräberfeldes wird von der Zahl der Kurgane bzw. der Ketten bestimmt. Die flächenmäßig kleinsten Gräberfelder mit Großkurganen im südöstlichen Siebenstromland, z. B. zwei Kurgane 400 m westlich vom Dorf Žalauly396 oder die Kurgankette Krasnyj Vostok397, haben eine Größe von II $8 \times 60 \mathrm{~m}$ bzw. I02 $\times$ I60 m, also eine Fläche von $7080 \mathrm{~m}^{2}$ bzw. I6 $320 \mathrm{~m}^{2}$. Zudem gibt es eine Vielzahl von Gräberfeldern, die nur aus einer Kette bestehen und trotz der relativ bescheidenen Anzahl der Grabhügel große Flächen beanspruchen $3^{8}$. Ferner kamen im Untersuchungsgebiet auch gigantische Nekropolen vor, die ein Ausmaß von 8,6 × 10 km (Gf. Ereul399) oder 16,2 × 16,6 km (Gräberfeld auf dem Plateau Kegen400) erreichten und die somit eine Fläche von $86000000 \mathrm{~m}^{2}\left(86 \mathrm{~km}^{2}\right)$ bzw. $268920000 \mathrm{~m}^{2}$ (ca. $267 \mathrm{~km}^{2}$ ) einnahmen.

Mehr als eine Kette wiesen I9 untersuchte Gräberfelder im Forschungsgebiet auf. Der Abstand zwischen den einzelnen Ketten ist unterschiedlich und variiert zwischen 3-I3 m (Gf. Žylysaj-I40I) und 3,0-I2, O km (Gf. auf dem Plateau Kegen ${ }^{402}$ ). Die durchschnittliche Entfernung zwischen den einzelnen Ketten innerhalb einer Nekropole beträgt I, $3 \mathrm{~km}$. In der Regel stehen die Tumuli jedoch zwischen Io० $\mathrm{m}$ und $500 \mathrm{~m}$ voneinander entfernt403. Wie das Balkendiagramm (Abb. 80) zeigt, kommen am häufigsten die Gräberfelder vor, die nur aus einer Kurgankette bestehen. Etwas seltener trifft man auf einem Gräberfeld zwei oder fünf Ketten an. Es folgen die Nekropolen mit drei und vier Ketten. In je einem Falle sind Gräberfelder mit sieben bzw. elf Ketten bekannt.

Hierbei muss bemerkt werden, dass die Nekropole Taučilik-6 aus zwei Großkurganen, die eine Kette bildeten, und aus I44 Kleinkurganen, die die restlichen zehn Ketten ausmachen, besteht. Als das Gräberfeld mit der größten Anzahl an Kurganketten mit Großkurganen ist die Nekropole Issyk hervorzuheben. Sie besteht aus sieben Ketten.

Eine Kette kann zwischen zwei404 und 38 Kurganen 405 aufweisen und unterschiedlich ausgerichtet worden sein. Das Diagramm auf Abbildung 8I zeigt, dass die Ausrichtung der Ketten in einer Nord-

Es handelte sich dabei um 29 Gräberfelder, die insgesamt 743 Kurgane aufwiesen, welche sich auf 85 Kurganketten verteilen.

396 Siehe Katalog, Abb. Io8.

397 Siehe Katalog, Abb. I59.

398 z. B.: Gf. Ulžan misst $640 \times 80 \mathrm{~m}$. Die gesamte Fläche der Nekropole beträgt $5^{1200 ~ m^{2}}$ (siehe Katalog, Abb. I94).

399 Siehe Katalog, Abb. I36-I39.

400 Siehe Katalog, Abb. I48.

4 OI Siehe Katalog, Abb. 210.
402 Siehe Katalog, Abb. I48.

403 Gräberfelder Bes Tobe (siehe Katalog, Abb. I28), Bestobe-3 (siehe Katalog, Abb. 132), Boroldaj (siehe Katalog, Abb. I35), Issyk (siehe Katalog, Abb. I43), Taučilik-6 (siehe Katalog, Abb. I79), Žalauly-2 (siehe Katalog, Abb. I96) und Žoan Tobe (siehe Katalog, Abb. 202) sowie die Nekropole des archäologischen Komplexes Kiikpaj (siehe Katalog, Abb. 2I7).

404 Zum Beispiel Gf. Bestobe-3 (siehe Katalog, Abb. 132) oder Gf. Žoan Tobe (siehe Katalog, Abb. 202).

405 Zum Beispiel Gf. Žalauly-2 (siehe Katalog, Abb. I96). 


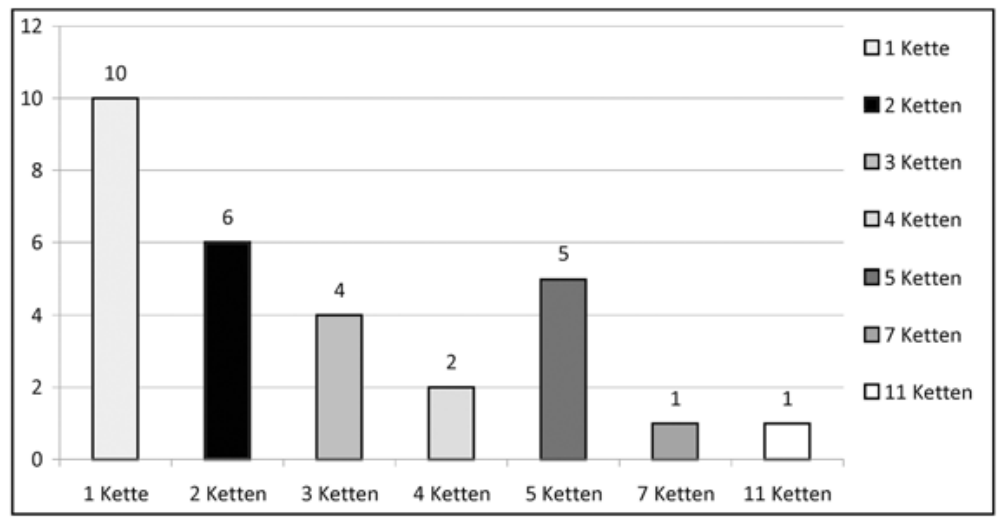

Abb. 8o | Anzahl der Kurganketten pro

Gräberfeld

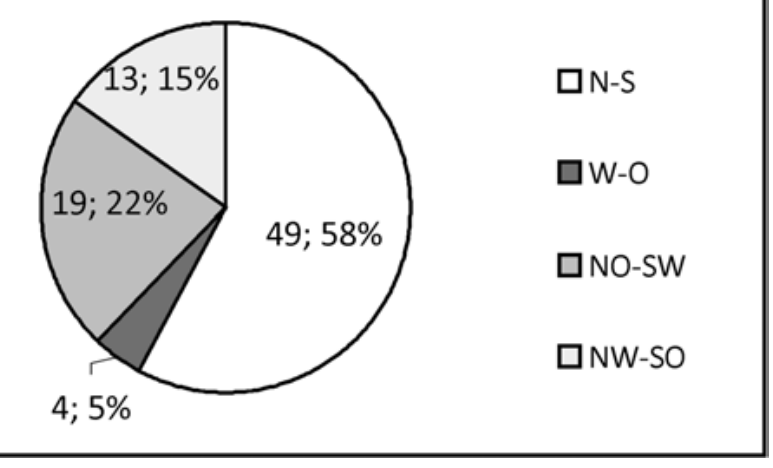

Abb. 81 | Die Ausrichtung der Kurganketten im

Untersuchungsgebiet

Süd-Achse mit $58 \%$ aller untersuchten Fälle (49 Kurganenketten) dominiert. Zudem treten die Nordost-Südwest- (22\%; I9 Ketten) und die Nordwest-Südost-Ausrichtung (15\%; I3 Fälle) auf. Am seltensten ist die Ausrichtung der Ketten in einer West-Ost-Achse, nämlich nur viermal406.

Die Ausrichtung der Ketten war vor allem von der Landschaftsbeschaffenheit abhängig. Da fast alle sakischen Gräberfelder mit Großkurganen auf Flussuferterrassen und die übrigen auf hochgelegenen Riedelkanten errichtet worden waren, richtete sich die Ausrichtung im Grunde nach einem bestimmten Geländeverlauf. Am besten wird diese Feststellung durch die Lage der Nekropole Turgen 407 unterstützt, die entlang des Flussufers angelegt ist und deren Ketten sogar den Verlauf der Flusswindungen wiederholen. Da alle Flüsse der nördlich von Transili-Alatau gelegenen Flachlandebene und auch einige auf dem Plateau Kegen von Süden nach Norden fließen, sind dementsprechend auch die Ketten der Gräberfelder ausgerichtet408. Die Nekropolen auf der Nordhälfte des Plateaus Kegen - Žalauly-2409 und Ža-

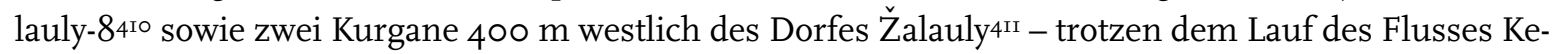
gen, der an dieser Stelle von Osten nach Westen fließt und wurden auf den von Norden nach Süden verlaufenden Riedelkannten, wie die meisten Gräberfelder des Forschungsgebietes, angelegt. Im Čilik-

406 Zwei Ketten auf dem Gf. Issyk (siehe Katalog, Abb. I43), eine Kette auf der Nekropole des Plateaus Kegen (siehe Katalog, Abb. I48, I5I) und die Kurgangruppe Krasnyj Vostok (siehe Katalog, Abb. I59).

407 Siehe Katalog, Abb. I84.
408 Welcher Kurgan als erster in der Kette errichtet worden ist, konnte anhand der Oberflächenuntersuchungen der Konstruktionen nicht geklärt werden.

409 Siehe Karte ir Nr. 62 und Katalog, Abb. I96.

4IO Siehe Karte ir Nr. 60 und Katalog, Abb. 200-20I.

4II Siehe Karte ir Nr. 64 und Katalog, Abb. Io8. 
Tal wiesen drei Kurgane, 7 km westlich vom Dorf Saty4² , eine Nord-Süd-Achse auf, die quer zum Flusslauf des Čilik verläuft. Die Ausrichtung der Ketten an der Nordost-Südwest- und der Nordwest-SüdostAchse entsprechen dem Verlauf der nahegelegenen Flüsse.

In allen vier Fällen von westöstlich orientierten Ketten wurden die Kurgane quer zum Flusslauf positioniert. Folglich stellen die zwei Nordketten des Gräberfeldes Issyk, die Kurgangruppe Krasnyj Vostok und die mittlere Kette auf dem Plateau Kegen eine Ausnahme dar. Andere Kurganketten des Gräberfeldes Issyk und des Plateaus Kegen vollziehen den „klassischen Verlauf“ von Norden nach Süden mit gewissen Abweichungen entlang eines Flusses nach. Es ist jedoch zu bedenken, dass diese drei Fundorte stark durch den Pflug beschädigt wurden. Zudem ist die Nekropole Issyk zum Teil modern bebaut, so dass fast alle Kleinkurgane dieser Gräberfelder komplett zerstört sind. Daher ist nicht sicher zu sagen, ob die westöstlich ausgerichtete Kurgankette nicht ursprünglich mehrere nordsüdlich verlaufende Ketten darstellte, die aus einem heute erhaltenem Großkurgan und mehreren zerstörten Kleinkurganen bestand.

Außer innerhalb der Kurganketten befanden sich in sieben Nekropolen zusätzlich einzeln stehende Großkurgane4³. Die Gräberfelder mit solchen Kurganen sind über das gesamte Untersuchungsgebiet verteilt. Es handelte sich dabei um ein bis vier Kurgane, die räumlich separiert von den Ketten gebaut worden waren. Auf den Gräberfeldern Kyzylšaryk414, Saty415 und der Nekropole auf dem Plateau Kegen ${ }^{4}{ }^{6}$ waren diese allein stehenden Kurgane gleichzeitig die größten Grabhügel des Fundortes. Die Lage solcher Kurgane im Bereich eines Gräberfeldes war unterschiedlich. Nur zwei Kurgane4r7 befanden sich im südwestlichen Bereich, am äußersten Rande des Gräberfeldes. Alle anderen Lagevarianten kamen nur jeweils einmal vor - nämlich in der Nord- bzw. Osthälfte der Nekropole sowie im nordwestlichen, südöstlichen oder nordöstlichen Bereich des Gräberfeldes.

\subsubsection{Lage der größten Kurgane innerhalb einer Nekropole und innerhalb einer Kette}

Die Lage der größten Kurgane auf den verschiedenen Gräberfeldern scheint nicht standardisiert gewesen zu sein. Wie die Abbildung 82 zeigt, wurden die größten Grabhügel in den meisten Fällen auf der Nord- (zehn Fälle4ז8; $35 \%$ aller untersuchten Gräberfelder) oder der Südhälfte (fünf Fälle4r9; I7\%) aber auch im Zentrum der Nekropole (sechs Fälle420; 2I \%) errichtet. Hervorzuheben ist, dass auf dem Plateau Kegen, das zur zweiten Verbreitungszone zählt, der größte Kurgan nur ein einziges Mal im Zen-

4I2 Siehe Karte II Nr. 43 und Katalog, Abb. IIo-III.

4 I3 Gf. Akšoky-4 KI (siehe Karte II Nr. 58 und Katalog, Abb. II6); Gf. Ereul K7-Kio (siehe Karte in Nr. 69 und Katalog, Abb. I38-I39); Gf. auf dem Plateau Kegen KI und K2 (siehe Karte iI Nr. 68 und Katalog, Abb. I48-I49); Gf. Kyzylšaryk Kio (siehe Karte iı Nr. 28 und Katalog, Abb. I64,I); Gf. Nordwestlicher Friedhof K4 (siehe Karte II Nr. I und Katalog, Abb. I66,I); Gf. Novoalekseevka KooI-003 (siehe Karte II Nr. Io und Katalog, Abb. I67); Gf. Saty K5 (siehe Karte II Nr. 45 und Katalog, Abb. I74).

4I4 Siehe Katalog, Abb. I64,I.

4I5 Siehe Katalog, Abb. I74.

4I6 Siehe Katalog, Abb. I48-I49.

4I7 K5 Gf. Saty und Kio Gf. Kyzylšaryk.
4I8 Drei Kurgane $7 \mathrm{~km}$ westlich vom Dorf Saty (Karte II Nr. 43), Gräberfelder Aksaj-9 (Karte in Nr. 56), Asy Saga (Karte II Nr. 29), Bes Tobe (Karte II Nr. 22), Bestobe-3 (Karte II Nr. 59), Ereul (Karte II Nr. 69), Issyk (Karte II Nr. I4), Nordwestlicher Friedhof (Karte II Nr. I), Novoalekseevka (Karte II Nr. Iо) und Togyzbulak (Karte II Nr. 46).

4I9 Zwei Kurgane $400 \mathrm{~m}$ westlich vom Dorf Žalauly (Karte II Nr. 64), Nekropole des archäologischen Komplexes Kiikpaj (Karte II Nr. 24), Gräberfelder Turgen (Karte II Nr. I9), Ulžan (Karte II Nr. 3) und Žylysaj-I (Karte II Nr. 50).

420 Gräberfelder Akšoky-4 (Karte II Nr. 58), Kokpijaz (Karte II Nr. 66), Sarytau „A“ (Karte II Nr. 70), Šyrganak-2 (Karte II Nr. 67), Žalauly-2 (Karte II Nr. 62) und Žalauly-8 (Karte II Nr. 6o). 


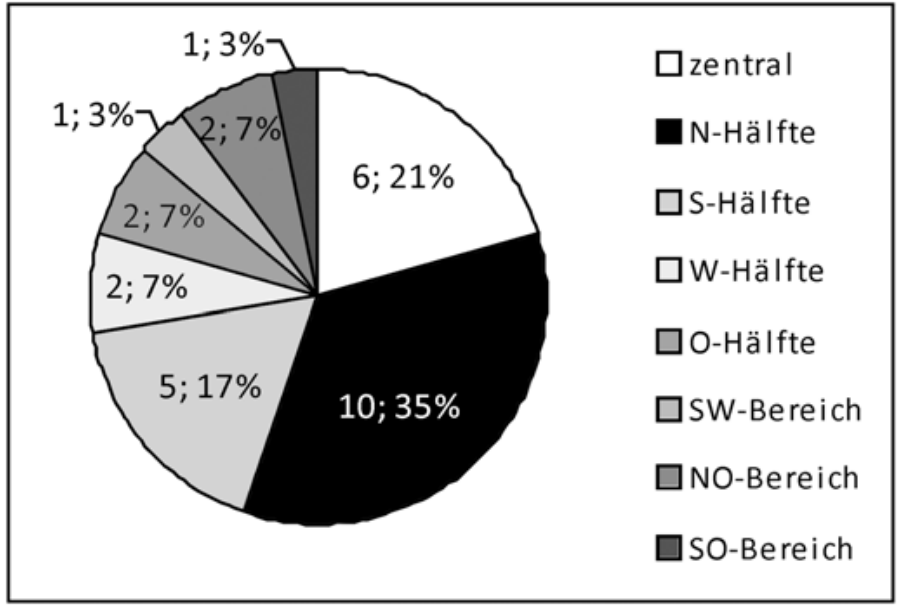

Abb. 82 | Lage des größten Kurgans innerhalb einer Nekropole

trum einer Nekropole nachgewiesen wurde. Hingegen ist die Lage in der nördlichen und südlichen Hälfte der Nekropole für das gesamte Untersuchungsgebiet belegt. Dies könnte damit erklärt werden, dass die meisten Ketten in einer Nord-Süd-Achse verlaufen.

Es konnte festgestellt werden, dass sich der größte Kurgan in sechs Fällen ${ }^{42 \mathrm{I}}$ auf insgesamt zehn Gräberfeldern in der Nordhälfte befand und zudem eine periphere Lage auf der Nekropole einnahm. Auf fünf Gräberfeldern wurde der größte Kurgan in der Südhälfte errichtet, befand sich allerdings nur in drei Fällen am äußersten Rande des Friedhofes422.

Die Lage des größten Kurgans in der Osthälfte der Nekropole wurde auf den Gräberfeldern Aktasty-3 (KI, siehe Karte II Nr. 74 und Katalog, Abb. II8) und auf dem Plateau Kegen (K2, siehe Karte II Nr. 68 und Katalog, Abb. I48) festgestellt (7\%). Auf dem Plateau Kegen war der größte Kurgan auch der am äußersten Rand gelegene. Bei zwei weiteren Nekropolen (7\%) trat der größte Kurgan in der Westhälfte auf (Kurgangruppe Krasnyj Vostok, KI ${ }^{423}$ und Gf. Taučilik-6, KI'24). Der Kurgan I der Kurgangruppe Krasnyj Vostok nahm zudem die äußerste Position auf der Nekropole ein. Es soll jedoch bemerkt werden, dass es sich bei beiden Gräberfeldern jeweils nur um zwei Großkurgane handelte. Daher scheint die Platzierung des größten Kurgans in der westlichen Hälfte der Nekropole nicht aussagekräftig zu sein. Zwei weitere Gräberfelder $(7 \%)$ wiesen den größten Kurgan der Nekropole im nordöstlichen Bereich auf (Gf. Boroldaj, Ki6425 und Gf. Žoan Tobe, KI ${ }^{426}$ ). Jeweils einmal trat der größte Kurgan im südöstlichen (Gf. Saty, K5 ${ }^{427}$ ) bzw. südwestlichen Bereich (Gf. Kyzylšaryk, KIO428) des Gräberfeldes auf.

Die Großkurgane der Nekropole, deren Höhe über $2 \mathrm{~m}$ reichte, wurden ebenso wie die größten Kurgane des Fundortes unterschiedlich platziert. Die Lagevarianten der Großkurgane wiederholen sich bei den größeren Kurganen im gesamten Untersuchungsgebiet. Lageregeln für Mikroregionen lassen sich nicht erkennen. Da es sich dabei um einmalige oder zweimalige Erscheinungen handelte, sind sie

42I Kurgan I der drei Kurgane 7 km westlich vom Dorf Saty (siehe Katalog, Abb. IIo); Gf. Asy Saga, Ki (siehe Katalog, Abb. I22); Gf. Bes Tobe, K47 (siehe Katalog, Abb. I28); Gf. Bestobe-3, K6 (siehe Katalog, Abb. I32); Gf. Nordwestlicher Friedhof, KI (siehe Katalog, Abb. I66,I) und Gf. Togyzbulak, Ki (siehe Katalog, Abb. I8I).

422 Kurgan 2 der Zwei Kurgane 400 m westlich vom Dorf Žalauly (siehe Katalog, Abb. Io8); Nekropole des ar- chäologischen Komplexes Kiikpaj, K9 (siehe Katalog, Abb. 2I7) und Gf. Ulžan, Ki (siehe Katalog, Abb. I94).

423 Siehe Karte II Nr. I5 und Katalog, Abb. I59.

424 Siehe Karte II Nr. 42 und Katalog, Abb. I79.

425 Siehe Karte ir Nr. 2 und Katalog, Abb. 135,2.

426 Siehe Karte II Nr. 27 und Katalog, Abb. 202.

427 Siehe Karte II Nr. 45 und Katalog, Abb. I74.

428 Siehe Karte II Nr. 28 und Katalog, Abb. I64,I. 
Abb. 83 | Lage der Großkurgane innerhalb einerNekropole

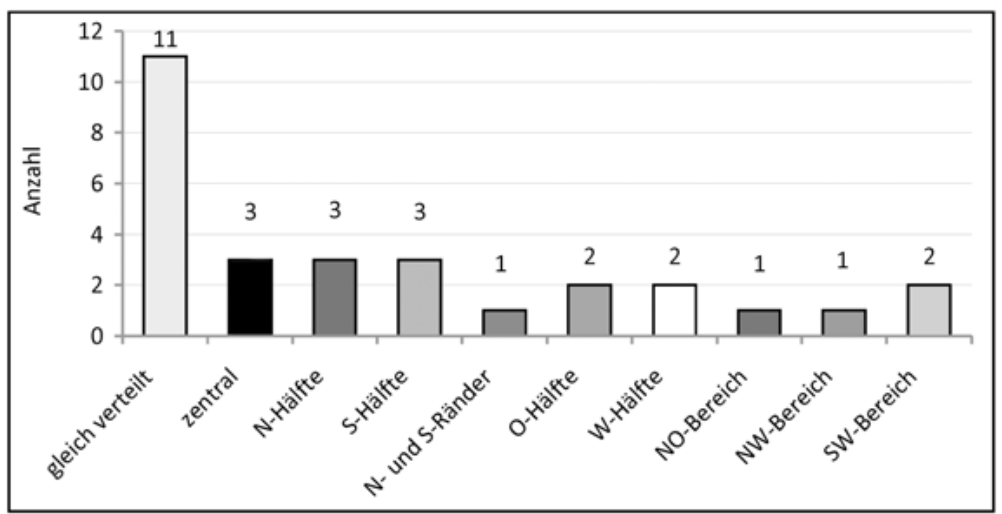

für die Analyse nicht repräsentativ. Wie das Diagramm in Abbildung 83 zeigt, befinden sich die Großkurgane meistens über das ganze Gräberfeld gleichmäßig verteilt (in elf Nekropolen429). Bei drei Gräberfeldern standen die Großkurgane jeweils einmal im Zentrum430, in der Nord-43 bzw. in der Südhälfte432 der Nekropole. Auf dem Gräberfeld Žoan Tobe befanden sich die Großkurgane nicht nur im mittleren Bereich, sondern auch in der Osthälfte. Ein weiterer Sonderfall stellte das Gräberfeld Bes Tobe dar433. Hier wurden die Großkurgane am südlichen und am nördlichen Rand errichtet. Der mittlere Bereich wies jedoch nur Kleinkurgane auf. Die Anlage von Großkurganen in der Ost-434 bzw. Westhälfte435 sowie im südwestlichen Bereich 436 des Gräberfeldes wurden bei jeweils zwei Nekropolen festgestellt. In der Nekropole Aktasty-3 bestand die ganze Ostkette aus den größten Kurganen des Gräberfeldes. Es gibt zudem wenige Beispiele, wo Großkurgane im nordöstlichen 437 und nordwestlichen 438 Bereich des Gräberfeldes zum Vorschein kamen.

Da die Ketten nicht aus exakt gleich großen Kurganen bestand, konnte in jeder Kette der größte Kurgan lokalisiert werden. Interessanterweise wiederholt sich die Lage des größten Kurgans innerhalb einer Kette in der Lage der größten Kurgane innerhalb eines Gräberfeldes (siehe Abb. 84; vgl. Abb. 82). So befindet sich beispielsweise auf dem Gräberfeld Žoan Tobe439 in der ersten Verbreitungszone der größte Kurgan der Nekropole auf der nördlichsten Position, ebenso finden sich die größten Tumuli in

429 Zwei Kurgane $400 \mathrm{~m}$ westlich vom Dorf Žalauly (siehe Karte II Nr. 64 und Katalog, Abb. Io8), drei Kurgane $7 \mathrm{~km}$ westlich vom Dorf Saty (siehe Karte in Nr. 43 und Katalog, Abb. IIo), Gräberfelder Asy Saga (siehe Karte II Nr. 29 und Katalog, Abb. I22), Bestobe-3 (siehe Karte II Nr. 59 und Katalog, Abb. 132), Kurgangruppe Krasnyj Vostok (siehe Karte II Nr. I5 und Katalog, Abb. I59), Gräberfelder Kyzylšaryk (siehe Karte II Nr. 28 und Katalog, Abb. I6I-I62), Novoalekseevka (siehe Karte II Nr. Io und Katalog, Abb. I67), Sarytau „A“ (siehe Karte II Nr. 70 und Katalog, Abb. I72), Šyrganak-2 (siehe Karte II Nr. 67 und Katalog, Abb. I76), Turgen (siehe Karte II Nr. I9 und Katalog, Abb. I84) und Ulžan (siehe Karte II Nr. 3 und Katalog, Abb. I94).

430 Gräberfelder Žalauly-2 (siehe Karte II Nr. 62 und Katalog, Abb. I96), Žoan Tobe (siehe Karte II Nr. 27 und Katalog, Abb. 202) und Žylysaj-I (siehe Karte II Nr. 50 und Katalog, Abb. 210).
43I Gräberfelder Ereul (siehe Karte II Nr. 69 und Katalog, Abb. I38-I39), Nordwestlicher Friedhof (siehe Karte II Nr. I und Katalog, Abb. I66,I) und Togyzbulak (siehe Karte iI Nr. 46 und Katalog, Abb. I8I).

432 Gräberfelder Boroldaj (siehe Karte II Nr. 2 und Katalog, Abb. I35), auf dem Plateau Kegen (siehe Karte II Nr. 68 und Katalog, Abb. I48) und Zalauly-8 (siehe Karte II Nr. 60 und Katalog, Abb. 200).

433 Siehe Katalog, Abb. I28.

434 Gräberfelder Akšoky-4 (siehe Katalog, Abb. Iı6) und Aktasty-3 (siehe Katalog, Abb. Iı8).

435 Nekropole des archäologischen Komplexes Kiikpaj (siehe Katalog, Abb. 2I7) und Gf. Kokpijaz (siehe Katalog, Abb. I6I,2).

436 Gf. Saty (siehe Katalog, Abb. I74) und Gf. Taučilik-6 (siehe Katalog, Abb. I79).

437 Gf. Issyk (siehe Katalog, Abb. I43).

438 Gf. Aksaj-9 (siehe Katalog, Abb. II2).

439 Siehe Karte II Nr. 27 und Katalog, Abb. 202. 


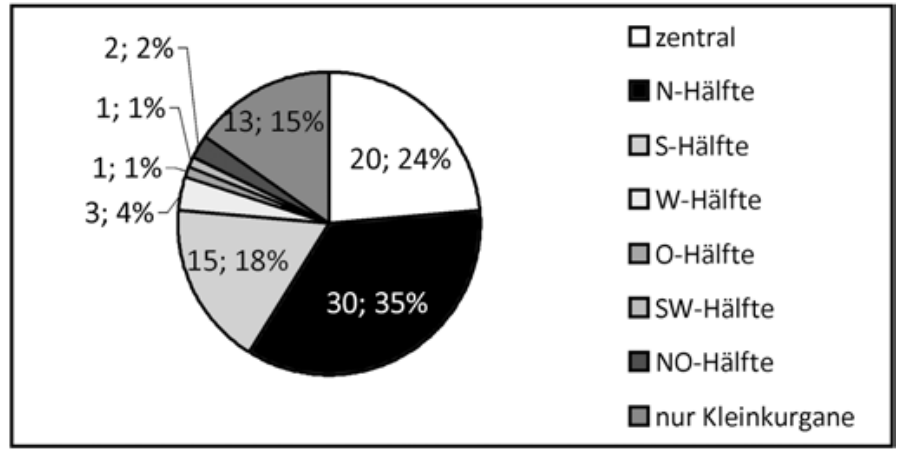

Abb. 84 | Lage des größten Kurgans innerhalb einer Kette
Abb. 85 | Lage der Großkurgane innerhalb einer Kette

allen drei Ketten am jeweils nördlichsten Rand. Auf dem Gräberfeld Žalauly-2440 in der zweiten Verbreitungszone steht der größte Kurgan im Zentrum der Nekropole und gleichzeitig im Zentrum der jeweiligen Kette.

Der größte Kurgan einer Kette konnte meistens in der Nord-44ז bzw. in der Südhälfte442 sowie im Zentrum443 lokalisiert werden. In sieben von 20 untersuchten Fällen, in denen sich der Kurgan im mittleren Bereich der Kette befand, stand der Kurgan exakt in der Mitte der Kette. In 30 Kurganketten wurde der größte Kurgan in der Nordhälfte errichtet; davon befand er sich in I8 Fällen am äußersten Ende der Kette. Bei elf Beispielen bildete der größte Kurgan das äußerste südliche Kettenende, obwohl in I5 Fällen der größte Kurgan innerhalb der Südhälfte der Kette angelegt wurde.

In drei Ketten wurde der größte Kurgan in der Westhälfte errichtet, in zwei weiteren Fällen in der nordöstlichen Hälfte. Jeweils einmal wurde der größte Kurgan der Kette in der Ost- bzw. Südwesthälfte festgestellt. In allen sieben Fällen nahm der größte Kurgan die äußerste Position in der Kette ein.

Falls sich mehrere Großkurgane innerhalb einer Kette befanden, wurden sie in der Regel gleichmäßig über die ganze Kette verteilt444 (Abb. 85). Wesentlich seltener traf man solche Kurgane in der Mitte der Kette oder in ihrer Nordhälfte an (in jeweils sieben Fällen445). Nur dreimal wurden die Großkurgane in der Südhälfte der Kette errichtet und einmal in der Osthälfte. Zusammenfassend lässt sich

440 Siehe Karte ir Nr. 62 und Katalog, Abb. I96.

44I 30 Kurganenketten; 35\% aller untersuchten Fälle.

442 I5 Kurganenketten; I8\% aller untersuchten Fälle.
44320 Kurganenketten; $24 \%$ aller untersuchten Fälle.

444 4I Kurganketten; $48 \%$ aller untersuchten Fälle.

445 In $7 \%$ aller untersuchten Fälle. 
Abb. 86| Lage der viereckigen Kurgane innerhalb einer Nekropole

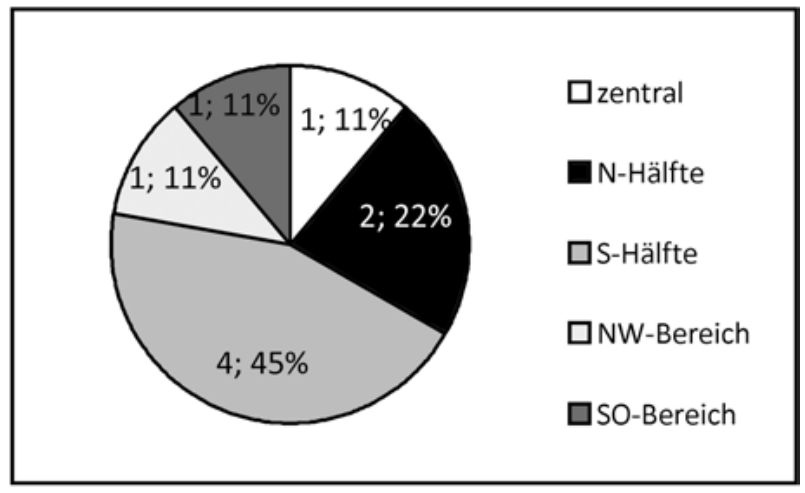

feststellen, dass die Lage der Großkurgane innerhalb einer Kette im Unterschied zu den größten Kurganen offensichtlich keine besondere Bedeutung besaß.

\subsubsection{Lage der viereckigen Kurgane}

Von I6 viereckigen, pyramidenförmigen Kurganen (Tabelle 24; Karte I7) konnten nur elf Kurgane von neun Gräberfeldern bezüglich ihrer Lage auf der Nekropole ausgewertet werden446. Der einzeln stehende Kurgan $5 \mathrm{~km}$ südwestlich des Dorfes Žalauly erbrachte diesbezüglich keine Information. Der Kurgan 6 in der Nekropole am Stausee Bartogaj befindet sich am Nordostufer und gehört zu einer noch sichtbaren Kette von Kleinkurganen. Da diese Kette aber nur einen Teil der Nekropole darstellt, kann auch dieses Beispiel nicht in die Analyse einbezogen werden. Die Lage von drei Kleinkurganen (K № 2, K № 3 und K № 7) des „kleinen Gräberfeldes“ der Nekropole Novoalekseevka (Kopylov I956, 3-5, II-2I, Taf. I, 5) kann heutzutage nicht mehr nachvollzogen werden.

Die Abbildung 86 zeigt, dass fast in der Hälfte der Fälle die viereckigen pyramidenförmigen Kurgane des gesamten Untersuchungsgebietes in der Südhälfte der Nekropole errichtet wurden (vier Gräberfelder447; $45 \%$ aller untersuchten Fälle). In der Nekropole Taučilik- 6448 befand sich ein solcher Kurgan auf der äußersten, südlichen Position. Auf zwei Gräberfeldern449 (22\%) wurden die viereckigen Kurgane in der Nordhälfte festgestellt. Der Kurgan 4 des Gräberfeldes Novoalekseevka erwies sich als der am nördlichsten gelegene Grabhügel. Jeweils in einem Fall traf man einen pyramidenförmigen Kurgan in der Mitte der Nekropole450 oder im nordwestlichen45 bzw. südöstlichen452 Bereich an.

Hervorzuheben ist, dass die viereckigen Kurgane nie die größten Kurgane eines Gräberfeldes darstellen. Dennoch gehören sie zur Kategorie „Großkurgane“.

446 Bei der Auswertung der viereckigen Kurgane innerhalb einer Nekropole wurde nicht die Anzahl der Kurgane, sondern die Häufigkeit des Vorkommens der Gräberfelder mit solchen Kurganen untersucht.

447 Gf. Bes Tobe, K2 (siehe Karte II Nr. 22 und Katalog, Abb. I28); Gf. Taučilik-6, KI (siehe Karte II Nr. 42 und Katalog, Abb. I79); Gf. Žoan Tobe, K2, K6 und K8 (siehe Karte II Nr. 27 und Katalog, Abb. 202) und Gf. Žylysaj-I, K6 (siehe Karte II Nr. 50 und Katalog, Abb. 210).

448 Siehe Katalog, Abb. I79.
449 Gf. Asy Saga, K3 (siehe Karte II Nr. 29 und Katalog, Abb. I22) und Gf. Novoalekseevka, K4 (siehe Karte II Nr. Io und Katalog, Abb. I67).

450 Gf. Novoalekseevka, K Io (siehe Karte ir Nr. Io und Katalog, Abb. I67).

45I Gf. Turgen, Ki2 (siehe Karte II Nr. I9 und Katalog, Abb. I84).

452 Gf. Kyzylšaryk, K2 (siehe Karte II Nr. 28 und Katalog, Abb. I6r). 
Ein pyramidenförmiger Kurgan war nur einmal Bestandteil einer Kette453. Eine Nekropole kann allerdings mehrere solche Kurgane aufweisen. Auf dem Gräberfeld Novoalekseevka, das heute aus zwei Ketten besteht, nahmen die viereckigen Kurgane (K4 und Kıo)454 sogar die gleiche Position innerhalb der beiden Ketten ein, nämlich die äußerste nördliche.

Innerhalb einer Kurgankette befanden sich die viereckigen Kurgane zu gleichen Teilen (46\%) entweder in der Süd- (auf fünf Gräberfeldern) oder in der Nordhälfte (auf fünf Gräberfeldern). Dabei stand in drei Fällen ein solcher Kurgan als äußerster, nördlicher und in zwei Fällen als äußerster, südlicher. Kurgan 6 des Gräberfeldes Žylysaj-I wurde im mittleren Bereich der Kette errichtet.

Ein regionales Verteilungsmuster der viereckigen Kurgane innerhalb einer Nekropole wurde nicht festgestellt.

\subsubsection{Lage der Kurgane mit Peripheriekonstruktionen}

Im gesamten Untersuchungsgebiet wurden 22 Kurgane auf elf Gräberfeldern mit Peripheriekonstruktionen festgestellt (Tabelle I6; Karte I2). Mit einer Ausnahme, nämlich dem einzeln stehenden Kurgan $5 \mathrm{~km}$ südwestlich des Dorfes Žalauly, wurden solche Anlagen in Bezug auf ihre Lage innerhalb eines Gräberfeldes ausgewertet455.

Fast in der Hälfte der Fälle (46\%; auf fünf Gräberfeldern ${ }^{46}$ ) befanden sich solche Kurgane im mittleren Bereich der Nekropole (Abb. 87). Die äußerste, nördliche Position hingegen nahm Kurgan I des Gräberfeldes Asy Saga mit Peripheriekonstruktionen ein. Jeweils auf zwei Gräberfeldern (I8\%) wurden solche Kurgane in der Osthälfte457 bzw. im nordöstlichen Bereich $45^{8}$ des Gräberfeldes festgestellt. Ferner standen die Kurgane II, I4 und I5 des Gräberfeldes Kyzylšaryk459 im südwestlichen Bereich.

Bezüglich der Lage der Kurgane mit Peripheriekonstruktionen innerhalb einer Kette konnte festgestellt werden, dass sich das Verteilungsmuster innerhalb einer Kette von dem Verteilungsmuster innerhalb einer Nekropole nicht wesentlich unterscheidet. Als Hauptpositionierung für solche Kurgane kann die Mitte (fünf Fälle; $42 \%$ aller untersuchten Ketten mit den Kurganen mit Peripheriekonstruktionen) bzw. die Nordhälfte (vier Fälle; $33 \%$ ) der Kette bezeichnet werden. Nur in zwei Fällen (I7\%) wurden solche Grabhügel in der Südhälfte und in einem Fall in der Osthälfte der Kette errichtet. In den Fällen, in denen sich die Kurgane in der Nord- und Osthälfte einer Kette befanden, standen sie an der äußersten Position. Innerhalb einer Kurgankette wurden ein 460 bis drei Kurgane mit Peripheriekonstruktionen46r festgestellt.

453 Siehe z. B. Gf. Žoan Tobe (Katalog, Abb. 202).

454 Siehe Katalog, Abb. I67.

455 Bei der Auswertung der Kurgane mit Peripheriekonstruktionen innerhalb einer Nekropole wurde nicht die Anzahl der Kurgane, sondern die Häufigkeit des Vorkommens der Gräberfelder mit solchen Kurganen untersucht.

456 Gf. Aksaj-9, K8, Kio (siehe Karte ir Nr. 56 und Katalog, Abb. II2); Gf. Asy Saga, K4 (siehe Karte II Nr. 29 und Katalog, Abb. I22); Gf. Issyk, K9, K2I, K22, K30 und K3I (siehe Karte Ir Nr. 45 und Katalog, Abb. I43); Gf. Turgen, $\mathrm{K}_{3}$ und $\mathrm{K}_{4}$ (siehe Karte II Nr. I9 und Katalog, Abb. I84) und Gf. Žylysaj-I, K6, K7 und K9 (siehe Karte II Nr. 50 und Katalog, Abb. 210).
457 Gf. Aktasty-3, K4 (siehe Karte II Nr. 74 und Katalog, Abb. II8) und Gf. Žalauly-2, K6I (siehe Karte II Nr. 62 und Katalog, Abb. I96).

458 Gf. Boroldaj, Ki6 (siehe Karte II Nr. 2 und Katalog, Abb. 135,2) und Gf. Žoan Tobe, KI (siehe Karte II Nr. 27 und Katalog, Abb. 202).

459 Siehe Karte ir Nr. 28 und Katalog, Abb. I62.

460 z.B.: Gf. Žalauly-2, K6I (siehe Katalog, Abb. I96) oder Gf. Žoan Tobe, KI (siehe Katalog, Abb. 202).

46I z.B.: Gf. Žylysaj-I, K6, K7 und K9 (siehe Katalog, Abb. 2IO). 
Abb. 87| Lage der Kurgane mit Peripheriekonstruktionen innerhalb einer Nekropole

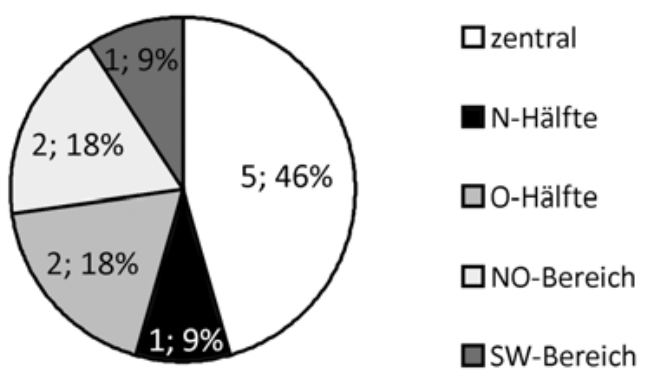

Peripheriekonstruktionen kommen sowohl innerhalb der Kette als auch innerhalb der Nekropole überwiegend im mittleren Bereich vor462. Zudem baute man sie um die größten Kurgane des Gräberfeldes. Folglich wurden die Peripheriekonstruktionen entweder um die größten Grabhügel herum oder um die in der Mitte des Gräberfeldes bzw. einer Kette gebauten Kurgane errichtet463.

Kurgane mit Peripheriekonstruktionen wurden im gesamten Untersuchungsgebiet festgestellt. Ein regionales Verteilungsmuster solcher Kurgane innerhalb einer Nekropole konnte nicht herausgearbeitet werden.

\subsubsection{Lage der Kurgane mit einem sog. Prozessionsweg}

Auf neun Gräberfeldern wurden ig Kurgane mit einem sog. Prozessionsweg festgestellt (Tabelle I7; Karte 13). Diese Grabhügel konnten, mit einer Ausnahme (Kurgan 6 vom Nordostufer der Nekropole am Stausee Bartogaj), bezüglich ihrer Lage in der Nekropole ausgewertet werden464. Da der Kurgan 6 des Gräberfeldes am Stausee Bartogaj nur zu einem nicht vollständigen Teil der Kette gehört, kann diese Kette aufgrund der fehlenden Informationen nicht in die Analyse einbezogen werden.

Wie die Abbildung 88 zeigt, gibt es keine eindeutig dominierende Lage solcher Kurgane auf den untersuchten Gräberfeldern. Am häufigsten treten Kurgane mit einem sog. Prozessionsweg im mittleren Bereich des Gräberfeldes auf (auf drei Nekropolen465; 37\% aller untersuchten Fälle). Jeweils auf zwei Gräberfeldern (je 25\%) wurden Kurgane mit einem sog. Prozessionsweg in der Ost-466 bzw. in der Nordhälfte467 der Nekropole festgestellt. Kurgan 4 des Gräberfeldes Aktasty-3 nahm den äußersten, östlichen Platz in der Nekropole ein. Die Kurgane I der Gräberfelder Asy Saga und Žoan Tobe beanspruch-

462 Fast die Hälfte aller untersuchten Fälle innerhalb einer Nekropole, nämlich $46 \%$, und $42 \%$ innerhalb einer Kette.

463 Als ein Beispiel für dieses Muster kann das Gf. Asy Saga erwähnt werden (siehe Katalog, Abb. I22). Hier befanden sich Peripheriekonstruktionen um den größten Kurgan des Gräberfeldes, KI, der auf der äußersten, nördlichen Position des Gräberfeldes stand. Kurgan 4 mit Peripheriekonstruktionen wurde im mittleren Bereich des Gräberfeldes lokalisiert.

464 Bei der Auswertung der Kurgane mit einem sog. Prozessionsweg innerhalb einer Nekropole wurde nicht die Anzahl der Kurgane, sondern die Häufigkeit von Gräberfeldern mit solchen Kurgane untersucht.
465 Gf. Aksaj-9, K8 (siehe Karte II Nr. 56 und Katalog, Abb. II2); Gf. Turgen, $\mathrm{K}_{3}$ und $\mathrm{K}_{4}$ (siehe Karte II Nr. I9 und Katalog, Abb. I84) und Gf. Žylysaj-I, K2, K3, K5, K6, K7, K9 und KII (siehe Karte ir Nr. 50 und Katalog, Abb. 2IO).

466 Gf. Aktasty-3, K4 (siehe Karte II Nr. 74 und Katalog, Abb. II8) und Gf. Žalauly-2, K6I (siehe Karte II Nr. 62 und Katalog, Abb. I96).

467 Gf. Asy Saga, KI, K3 und K4 (siehe Karte II Nr. 29 und Katalog, Abb. I22) und Gf. Žoan Tobe, KI (siehe Karte II Nr. 27 und Katalog, Abb. 202). 


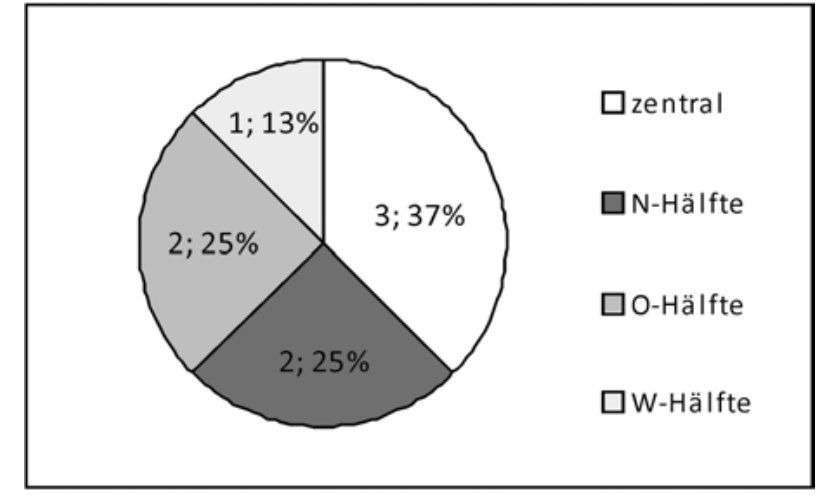

Abb. 88 | Lage der Kurgane mit einem sog. Prozessionsweg innerhalb einer Nekropole

ten die äußerste nördliche Position des Gräberfeldes. Die Kurgane mit einem sog. Prozessionsweg, die die äußerste Position innerhalb einer Nekropole einnahmen, waren auch gleichzeitig die größten Kurgane der jeweiligen Fundorte. Auf dem Gräberfeld Kyzylšaryk468 standen zwei Kurgane (KIo und KI4) mit einem sog. Prozessionsweg in der Westhälfte der Nekropole.

Innerhalb einer Kette traten zwischen einem ${ }^{469}$ und bis zu sieben 470 Kurgane mit einem sog. Prozessionsweg auf. Zu gleichen Teilen (38\%; jeweils auf drei Kurganketten) befanden sich solche Kurgane im mittleren Bereich oder in der Nordhälfte einer Kette. Außerdem wurden noch in zwei weiteren Ketten Kurgane mit einem sog. Prozessionsweg in der Südhälfte der Kette errichtet.

Trotz des Nachweises von Kurganen mit einem sog. Prozessionsweg in der südlichen Hälfte einer Kette treten solche Tumuli nie in der Südhälfte eines Gräberfeldes auf.

Die Kurgane mit einem sog. Prozessionsweg zeigten kein Verteilungsmuster in Bezug auf die Himmelsrichtungen oder die Kurgangröße. Ferner konnte auch kein regionales Verteilungsmuster solcher Kurgane auf den Nekropolen festgestellt werden. Folglich bleibt die Ursache für die Platzwahl der Anlagen mit einem sog. Prozessionsweg fraglich und die Klärung der Frage somit Aufgabe der zukünftigen Forschung.

\subsubsection{Lage der Kurgane mit einem Kreisgraben}

Auf sechs Gräberfeldern wurden insgesamt 28 Kurgane mit einem Kreisgraben festgestellt (Tabelle I9; Karte I4). Mit einer Ausnahme eines einzeln stehenden Kurgans, 5 km südwestlich des Dorfes Žalauly, konnten solche Anlagen bezüglich ihrer Lage innerhalb eines Gräberfeldes ausgewertet werden47I.

Die Abbildung 89 zeigt, dass sich zwei Lagetypen unterscheiden lassen. Die erste Gruppe stellen Kurgane der Nekropole Boroldaj 472 dar, die auf dem gesamten Gräberfeld gleichmäßig verteilt waren. Außer der nicht regelmäßigen Verteilung der Grabhügel auf dem Gräberfeld ist festzustellen, dass die

468 Siehe Karte II Nr. 28 und Katalog, Abb. I62 und Abb. I64,I.

469 z. B.: Gf. Aksaj-9, K8 (siehe Katalog, Abb. Iı2) oder Gf. Žoan Tobe, KI (siehe Katalog, Abb. 202).

470 z. B.: Gf. Žylysaj-I, K2, K3, K5, K6, K7, K9 und KII (siehe Katalog, Abb. 210).
47I Bei der Auswertung der Kurgane mit einem Kreisgraben innerhalb einer Nekropole wurde nicht die Anzahl der Kurgane, sondern die Häufigkeit des Vorkommens der Gräberfelder mit solchen Kurganen untersucht.

472 Insgesamt I7 Kurgane - KI, K5-K8, Kı。, KI5, KI7, Kı8, $\mathrm{K}_{24}-\mathrm{K}_{2} 6, \mathrm{~K}_{2} 8, \mathrm{~K}_{3} \mathrm{O}-\mathrm{K}_{33}$ und $\mathrm{K}_{4} \mathrm{O}$ (siehe Karte II Nr. 2 und Katalog, Abb. I35,2). 
Abb. 89 | Lage der Kurgane mit einem Kreisgraben innerhalb einer Nekropole

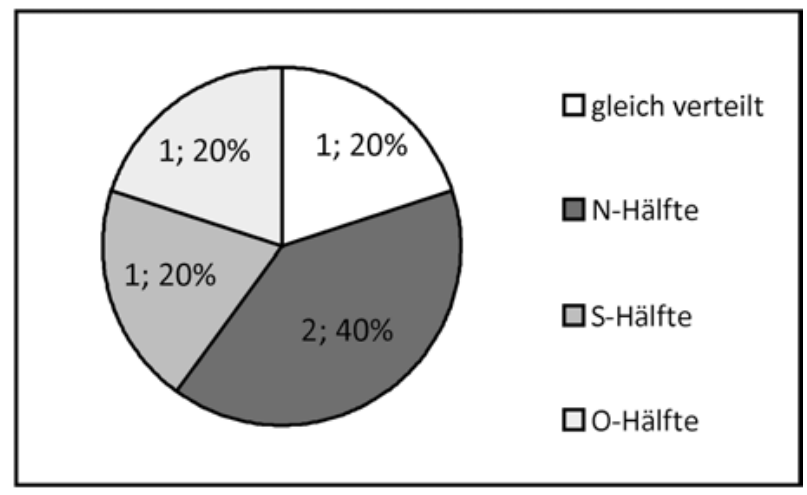

Kurgane der Nordhälfte der Nekropole oft außer einem Kreisgraben noch zusätzlich einen Erdwall aufweisen. In der südlichen Hälfte des Gräberfeldes kommen Kurgane mit einem Kreisgraben sowie solche mit einem Steinkreis in wechselnder Position vor. Diese Fundstelle ist die einzige Nekropole mit Kurganen und Kreisgraben, die in der ersten Verbreitungszone von früheisenzeitlichen Gräberfeldern mit Großkurganen, im nördlich des Transili-Alatau gelegenen Flachland liegt.

In der zweiten Verbreitungszone, in der Umgebung des Hochplateaus Kegen, traten solche Kurgane in bestimmten Bereichen auf, nämlich in der Nord-473, Süd-474 oder Osthälfte475 der Nekropole. Dabei können der Kurgan 35 des Gräberfeldes Ereul als äußerster östlicher Grabhügel und der Kurgan I der Nekropole Togyzbulak als äußerster nördlicher Kurgan betrachtet werden. Alle Kurgane mit einem Kreisgraben in der Nekropole Žalauly-8, die sich in der Nordhälfte des Fundortes befanden, gehörten zur Kategorie der Kleinkurgane.

Dabei spielte es gar keine Rolle, ob die Kreisgräben geschlossen waren oder ein- bzw. zweimal unterbrochen waren.

Innerhalb einer Kette wurden Kurgane mit einem Graben gleich häufig sowohl in der nördlichen als auch in der südlichen Hälfte der Kette platziert (jeweils drei Kurganketten; $28 \%$ aller untersuchten Ketten, in denen Kurgane mit einem Kreisgraben auftraten). Von den Kurganen mit einem Graben, die sich in der Südhälfte befanden, stand ein Kurgan am äußersten Rand der Kette. Zwei weitere Kurgane nahmen die äußerste nördliche Position innerhalb einer Kette ein. In zwei weiteren Ketten wurden die Kurgane mit einem Kreisgraben gleichmäßig über die gesamte Kette verteilt. In einer dieser Ketten wechselten sich die Kurgane mit einem Kreisgraben mit den Anlagen mit einem Steinkreis ab. In einem einzigen Fall befanden sich die Kurgane mit einem Kreisgraben in der Nordosthälfte der Kette.

Grabhügel mit einem Kreisgraben wurden im gesamten Untersuchungsgebiet festgestellt. In der ersten Verbreitungszone sind diese Kurgane gleichmäßig über das ganze Gräberfeld verteilt. In der zweiten Verbreitungszone ist die Anlage der Hügel mit Kreisgraben an eine bestimmte Himmelsrichtung gebunden.

473 Gf. Togyzbulak, Ki (siehe Karte II Nr. 46 und Katalog, Abb. I8I) und Gf. Žalauly-8, K2, K3, K5, K6, K7 und K9 (siehe Karte in Nr. 60 und Katalog, Abb. 200).
474 Gf. auf dem Plateau Kegen, K4 (siehe Karte II Nr. 68 und Katalog, Abb. I53).

475 Gf. Ereul, K35 (siehe Karte II Nr. 69 und Katalog, Abb. 139). 


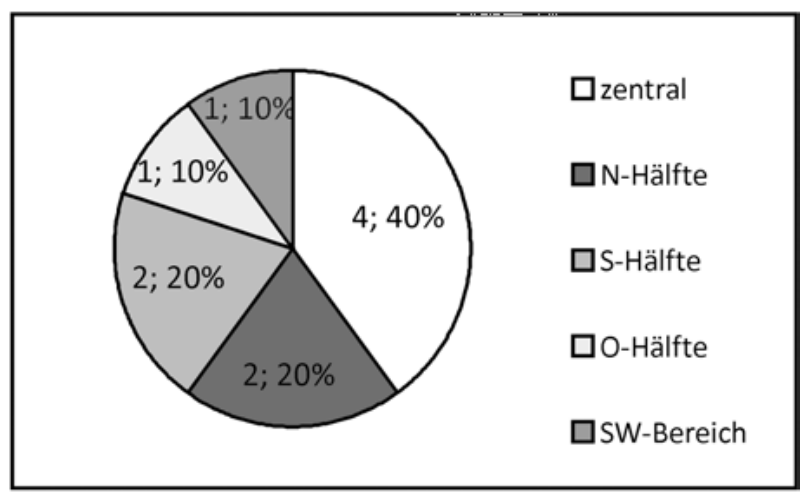

Abb. 9o | Lage der Kurgane mit einem Steinkreis innerhalb einer Nekropole

\subsubsection{Lage der Kurgane mit einem Steinkreis}

Von insgesamt 33 Kurganen mit Steinkreis, die sich auf neun Gräberfelder verteilten (Tabelle 2I; Karte I5), konnten nur 22 Grabhügel von acht Nekropolen ausgewertet werden $47^{6}$. Da das gesamte Gräberfeld von Sarytau, einschließlich der Kurgane mit einem Steinkreis, ausschließlich aus Kleinkurganen bestand, konnte dieser Fundort keinen Hinweis auf die Verteilung der Kurgane innerhalb eines Gräberfeldes mit Großkurganen liefern. Zudem konnte die Stelle des im Jahre I934 von R. Sulejmanov untersuchten „Karakemerskij Kurgans“ auf der Nekropole Turgen (Bernštam/Dublickij I936, 5-6) heute nicht mehr lokalisiert werden.

Die Abbildung 90 zeigt, dass fast die Hälfte der Kurgane mit einem Steinkreis im mittleren Bereich des Gräberfeldes steht (vier Gräberfelder477; 40\% aller untersuchten Fälle). Mit gleicher Zahl folgen dann die Gräberfelder, auf denen sich solche Kurgane entweder in der Nord-478 oder in der Südhälfte479 der Nekropole befinden (je zwei Nekropolen; je 20\%). Jeweils einmal traten Kurgane mit Steinkreis in der Osthälfte480 bzw. dem südwestlichen Bereich48r des Bestattungsplatzes auf.

Innerhalb einer Kette wurden ein ${ }^{42}$ bis fünf 483 Kurgane mit einem Steinkreis errichtet. Gleich häufig (jeweils 30\%; jeweils drei Ketten) traten solche Kurgane im mittleren Bereich oder in der Südhälfte einer Kette auf. Zudem wurden in zwei weiteren Ketten Kurgane mit einem Steinkreis in der Nordhälfte der Kette errichtet. Ferner wurden solche Kurgane jeweils in einer Kette in der Osthälfte, bzw. über die gesamte Kette gleichmäßig verteilt, festgestellt. Die Kurgane 25, 27 und 29 mit Steinkreis, die auf der gesamten Kette des Gräberfeldes Boroldaj gleichmäßig verteilt wurden, wechselten sich mit Kurganen mit Kreisgraben ab.

476 Beim Auswerten der Kurgane mit einem Steinkreis innerhalb einer Nekropole wurde nicht die Anzahl der Kurgane, sondern die Häufigkeit des Vorkommens der Gräberfelder mit solchen Kurganen untersucht.

477 Gf. Aktasty-3, K2I und K30 (siehe Karte in Nr. 74 und Katalog, Abb. II8); Gf. Turgen, K3 (siehe Karte II Nr. I9 und Katalog, Abb. I84); Gf. Žalauly-2, KI4, KI5 und KI9 (siehe Karte ir Nr. 62 und Katalog, Abb. I96) und Gf. Žylysaj-I, K8 (siehe Karte II Nr. 50 und Katalog, Abb. 2Iо).

478 Gf. Aksaj-9, K4, K7 und Kio (siehe Karte is Nr. ${ }_{5} 6$ und Katalog, Abb. II2) und Gf. Issyk, K8 (siehe Karte II Nr. I4 und Katalog, Abb. I43).
479 Gf. Boroldaj, K25, K27 und K29 (siehe Karte II Nr. 2 und Katalog, Abb. I35,2) und Gf. Žalauly-2, K2, $\mathrm{K}_{3}$ und $\mathrm{K}_{5} 6$ (siehe Karte II Nr. 62 und Katalog, Abb. I96).

480 Gf. Aktasty-3, KI, K2, K3 und K5 (siehe Karte II Nr. 74 und Katalog, Abb. II8).

48I Gf. Ereul, K20 (siehe Karte II Nr. 69 und Katalog, Abb. 139).

482 z.B.: Gf. Ereul, K20 (siehe Katalog, Abb. I39).

483 z.B.: Gf. Žalauly-2, K2, K3, KI4, KI5 und Ki9 (siehe Katalog, Abb. I96). 
Die Kurgane mit einem Steinkreis zeigten kein Muster im Bezug auf Orientierung oder Kurgangröße sowie keine regionalen Regeln bezüglich der Lage auf den untersuchten Nekropolen.

\subsubsection{Lage der Kurgane mit Sonderfällen}

Auf sechs Gräberfeldern, die auf das gesamte Untersuchungsgebiet verteilt sind, wurden 13 Kurgane mit verschiedenen Sonderfällen in ihrer Peripherie festgestellt (Tabelle 23; Karte I6). An der Nordseite des Kurgans 6 der Nekropole Asy Saga handelte es sich beispielsweise um eine Art „Vorsprung“. Der Kurgan nahm die südliche äußerste Stelle sowohl des gesamten Gräberfeldes als auch der Kurgankette ein 484 .

Der äußerste östliche Kurgan 2 des Gräberfeldes auf dem Plateau Kegen stand auf einer Plattform, von der noch die Überreste der Baurampen als strahlenförmig sichtbare Stege erhalten waren. Der Kurgan hatte keinen Bezug zu den Ketten der Nekropole485.

Auf zwei Gräberfeldern der ersten Verbreitungszone waren einige Kurgane von Erdwällen umgeben. Die Kurgane 5, 8-IO, I2, I3, 26 und 28 des Gräberfeldes Boroldaj befanden sich sowohl in der Nordhälfte der Nekropole als auch in der Nordhälfte der Ketten486. Dagegen stand der Kurgan 3 des Gräberfeldes Ulžan exakt in der Mitte der Kurgankette, die auch gleichzeitig die ganze Nekropole ausmachte487.

Das einzige Beispiel eines Kurgans mit Steinwall wurde bei Grabhügel 7 des Gräberfeldes Turgen festgestellt. Der Kurgan befand sich sowohl im mittleren Bereich der Nekropole als auch der Kette488.

In der zweiten Verbreitungszone der früheisenzeitlichen sakischen Gräberfelder mit Großkurganen wurde bei Flachkurgan 8 des Gräberfeldes Žalauly- 8 , am Nordrand des Plateaus Kegen, ein doppelter Steinkreis in der Nordhälfte der Kette und damit in der nördlichen Hälfte des Gräberfeldes festgestellt489.

Da es sich außer bei den Kurganen mit Erdwällen um einmalige Erscheinungen handelt, müssen Aussagen bezüglich der Platzwahl von Kurganen mit verschiedenen Sonderfällen in ihrer Peripherie innerhalb einer Kette als nicht aussagekräftig betrachtet werden.

Pro Kette treten zwischen einem490 und bis zu sechs49 Kurgane mit Erdwall auf, die entweder in der Nordhälfte oder in der Mitte einer Kette zu finden sind.

Zusammenfassend kann gesagt werden, dass die Anordnung der Kurgane auf einer Nekropole und deren spezifische äußere Form mehrere Parallelen auf den Gräberfeldern des skytho-sakischen Verbreitungsgebietes besitzt (vgl. Samašev u.a. 2000, Io; vgl. Korol'kova 2006, 25; vgl. Polin 2007, 256-267; vgl. Čugunov u.a. 20I0, I3; vgl. Samašev u.a. 20I0, 94). Die Kurgane bilden eine oder mehrere Kurganketten, die durch den Bezug zueinander als eine Nekropole zu verstehen sind. Die Ketten befinden sich an Flussuferterrassen oder an Riedelkanten und wiederholen in ihrer Anordnung den Reliefverlauf. Die meisten Ketten wurden entlang einer Nord-Süd-Achse ausgerichtet, was mit der Landschaftsbeschaffenheit des Untersuchungsgebiets zusammenhängt.

Außerhalb der Ketten können einzeln stehende Großkurgane vorkommen, die innerhalb der Grenzen eines Gräberfeldes unterschiedlich positioniert wurden.

484 Siehe Karte II Nr. 29 und Katalog, Abb. I22.

485 Siehe Karte ir Nr. 68 und Katalog, Abb. I48-I49.

486 Siehe Karte ir Nr. 2 und Katalog, Abb. I35,2.

487 Siehe Karte II Nr. 3 und Katalog, Abb. I94.

488 Siehe Karte II Nr. I9 und Katalog, Abb. I84.
489 Siehe Karte ir Nr. 6o und Katalog, Abb. 200.

490 z.B.: Gf. Ulžan, K3 (siehe Karte II Nr. 3 und Katalog, Abb. I94).

49I z. B.: Gf. Boroldaj, K5, K8, K9, Kıо, Kı2 und Kı3 (siehe Karte II Nr. 2 und Katalog, Abb. 135,2). 
Die Lage des größten Kurgans auf der Nekropole sowie innerhalb einer Kette deutet an, dass sie von der Ausrichtung der Ketten abhängig war. Bei der Platzwahl dominieren die Nordhälfte und der mittlere Bereich sowohl des Gräberfeldes als auch der Kette, dicht gefolgt von der Lage in der Südhälfte. Alle anderen Platzierungsvarianten stellten seltene oder gar einmalige Erscheinungen dar. Eine ähnliche Analyse zur Platzwahl der Großkurgane ergab, dass die Großkurgane in der Regel gleichmäßig verteilt waren und somit offenbar der Wahl des Platzes bei der Anlage des Hügels nur eine „zweitrangige“ Bedeutung zukam.

Viereckige, pyramidenförmige Kurgane traten in einer Kette nur einmal auf, obgleich manchmal mehrere solcher Kurgane auf einer Nekropole errichtet wurden. Pyramidenförmige Kurgane standen meistens in der Südhälfte der Nekropolen, innerhalb einer Kette konnten sie gleich häufig sowohl in der Nord- als auch in der Südhälfte lokalisiert werden.

Mehrere Kurgane mit Peripheriekonstruktionen wurden innerhalb einer Kette errichtet. Kurgane mit solchen Befunden stellen entweder die größten Kurgane der Nekropole dar, oder liegen im mittleren Bereich des Gräberfeldes bzw. einer Kette.

Ebenso kam vor, dass mehrere Kurgane mit einem sog. Prozessionsweg in einer Kette errichtet wurden. Die Analyse der Lage von solchen Kurganen innerhalb einer Nekropole und innerhalb einer Kurgankette zeigte keine Unterschiede sowohl im Bezug auf die Orientierung und die Kurgangröße als auch auf die regionalen Unterschiede innerhalb des Forschungsgebietes.

Dagegen wurden Kurgane mit einem Kreisgraben, die gleichmäßig über das gesamte Gräberfeld verteilt waren, nur in der ersten Verbreitungszone sakischer Nekropolen mit Großkurganen, nämlich in dem nördlich vom Transili-Alatau gelegenen Flachland, lokalisiert. In der zweiten Verbreitungszone befanden sich solche Kurgane nur in einem bestimmten Bereich der Nekropole, nämlich in der Nord-, Süd- oder Osthälfte.

Die Frage nach der Lage von Kurganen mit einem Steinkreis auf einem Gräberfeld muss zunächst unbeantwortet bleiben. Ähnlich wie bei Kurganen mit einem sog. Prozessionsweg konnten keine Platzierungsmarker festgestellt werden.

Dasselbe negative Ergebnis gilt für Kurgane mit Sonderfällen, da es sich meist nur um Einzelbeispiele handelte.

\subsection{Das Verhältnis der Nekropolen zueinander}

Auf allen untersuchten Gräberfeldern mit früheisenzeitlichen Großkurganen befanden sich meist abgerundete Kurgane, die zu Ketten aneinandergereiht waren. Fast alle Tumuli wurden auf ähnliche Weise gebaut und hatten eine fast identische äußere Form. Obwohl bei der Errichtung eines Grabhügels und der Planung der Nekropole eine gewisse Regelhaftigkeit zu erkennen ist, sind doch einige Besonderheiten zu beobachten.

Die Kurgane eines Gräberfeldes waren von unterschiedlicher Größe. Die statistische Analyse der Verteilung der Kurgane nach ihrer Größe erbrachte kein repräsentatives Ergebnis (siehe Kap. 5.I.I Groß- und Kleinkurgane). Ebenso ergebnislos blieb die im Streudiagramm dargestellte Größenanalyse aller Gräberfelder492 (Abb. 9I).

492 Bezüglich der Verteilung der Kurgane auf dem Gräberfeld wurden nur diejenigen Fundorte ausgewertet, die mehr als zwei Kurgane aufwiesen oder nicht nur aus
Kleinkurganen bestanden. So wurde der einzelne Kurgan, $5 \mathrm{~km}$ südwestlich vom Dorf Žalauly, zwei Kurgane, $400 \mathrm{~m}$ westlich vom Dorf Žalauly, das Gräberfeld am 


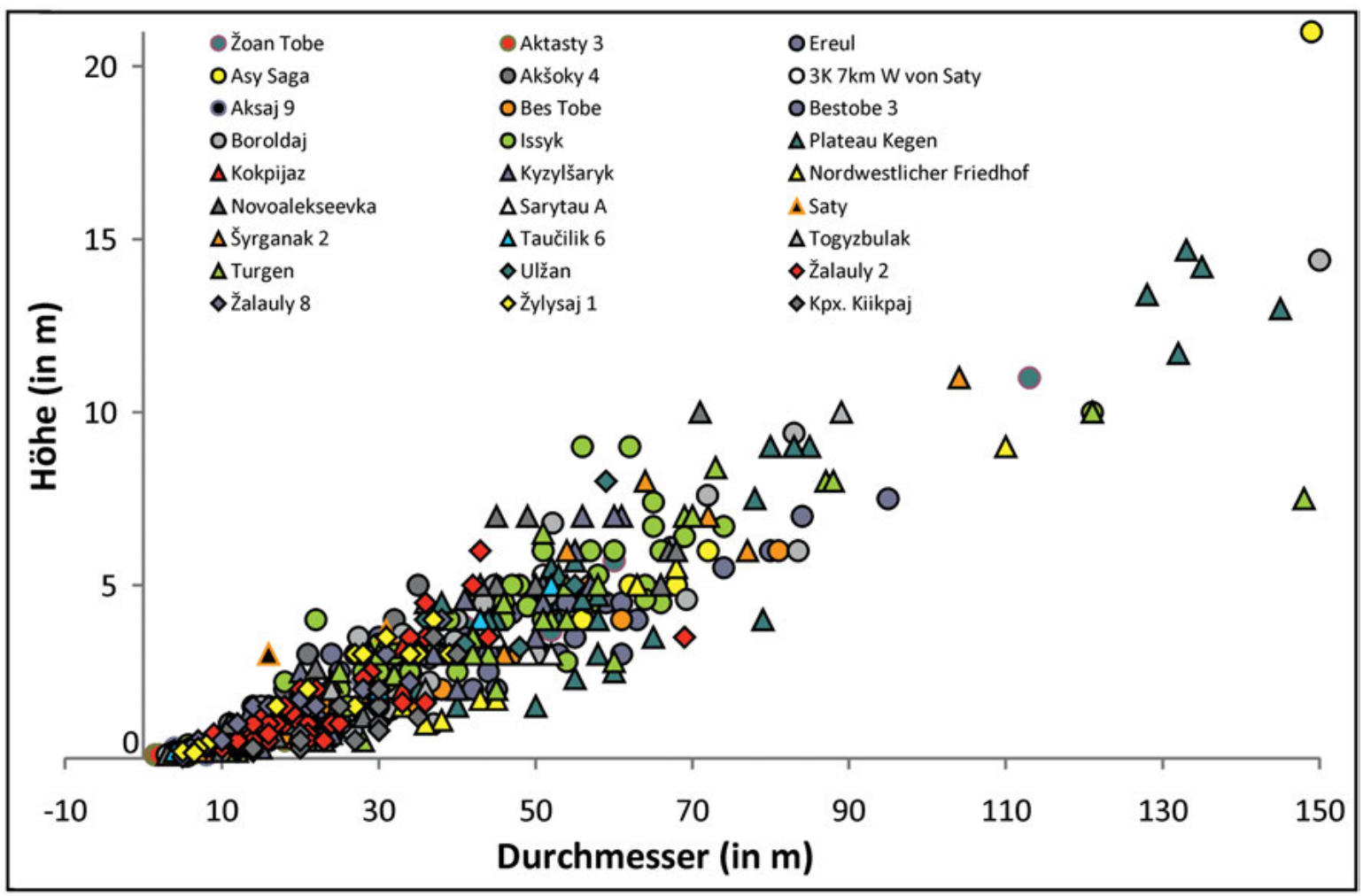

Abb. 91 | Gesamte statistische Verteilung aller Kurgane bezüglich ihrer Größe

Die Verteilung der Kurgane nach ihrer Größe auf die beiden Verbreitungszonen erbrachte ein Negativergebnis (Abb. 92-93).

Auch die statistische Verteilung der Größengruppen von Kurganen wurde für einige Gräberfelder einzeln erhoben und die Ergebnisse untereinander verglichen. Es stellte sich heraus, dass auch der Vergleich zwischen den Nekropolen kein Muster erkennen lässt (Abb. 94; Karte 3).

Die in der Abbildung 94 aufgeführten Gräberfelder im Gebiet zwischen dem Nordwestlichen Friedhof und der Fundstelle Žoan Tobe zählen zur ersten Verbreitungszone der sakischen Nekropolen mit Großkurganen. Die Gräberfelder auf der Graphik, die sich im Čilik-Tal zwischen den Fundstellen Taučilik- 6 und Saty befanden, wurden im Raum zwischen den beiden Verbreitungszonen erbaut. Die Nekropolen im Gebiet zwischen Togyzbulak493 und Aktasty-3 gehören der zweiten Verbreitungszone an.

Es gibt Gräberfelder, auf denen die Grabhügel eine nahezu gleiche Größe haben494, wobei die Ausmaße der Tumuli von Gräberfeld zu Gräberfeld unterschiedlich waren. Es wurden sechs Nekropolen mit fast gleichgroßen Kurganen festgestellt, was $22 \%$ aller untersuchten Gräberfelder ausmachte. Solche Nekropolen traten im gesamten Untersuchungsgebiet auf.

Stausee Bartogaj, die Kurgangruppe Krasnyj Vostok und die Nekropole des archäologischen Komplexes Sarytau nicht berücksichtigt.

493 Da die Gräberfelder Tugyzbulak, Žylysaj-I und Aksaj-9 sich außerhalb des Plateaus Kegen befinden, sind sie zuerst, von Westen nach Osten gegliedert, aufgeführt. Dann folgen die Nekropolen vom Plateau Kegen von Westen nach Osten.

494 Gräberfelder Ulžan, drei Kurgane 7 km westlich vom Dorf Saty, Saty, Bestobe, Kokpijaz und Sarytau „A“. 


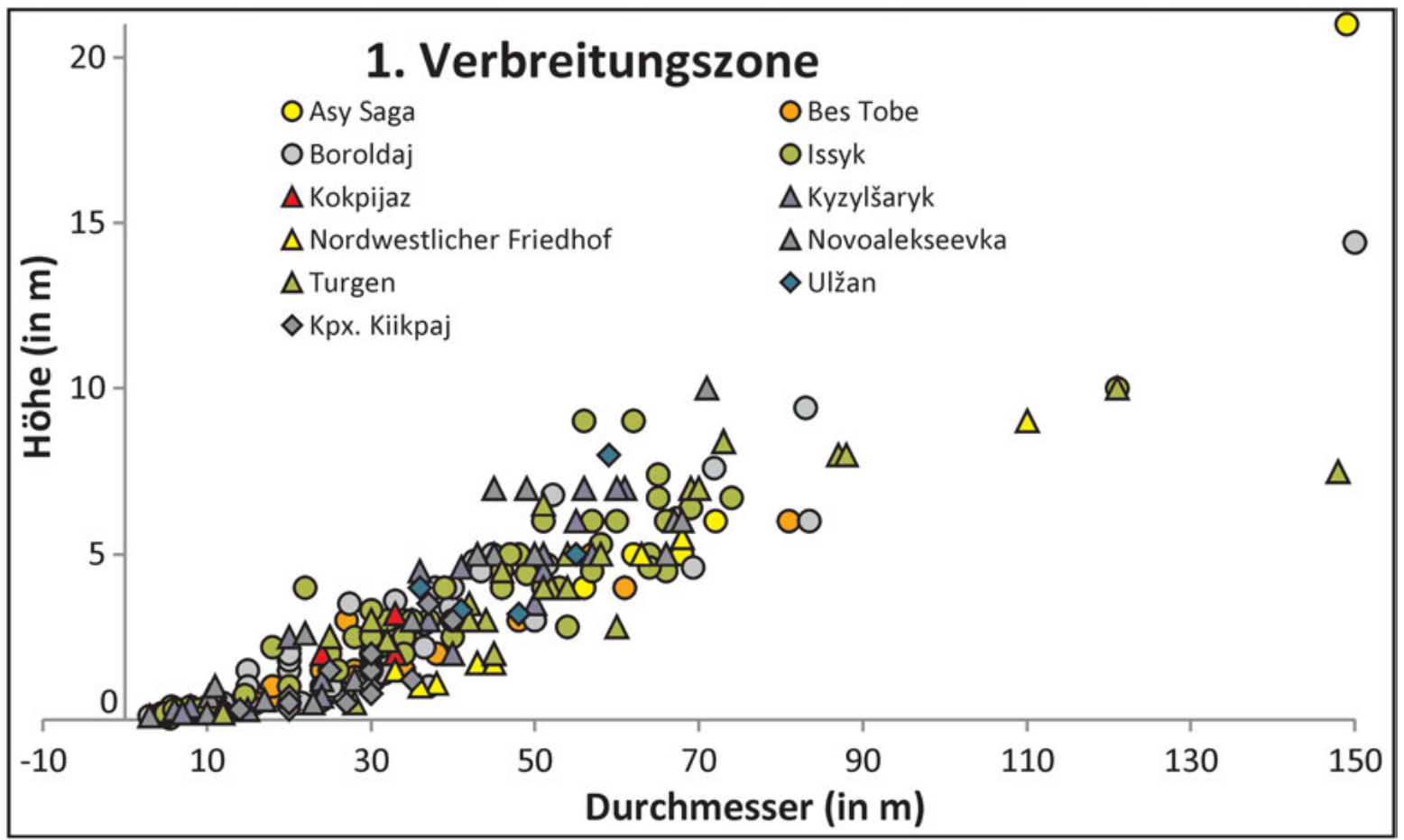

Abb. 92 | Gesamte statistische Verteilung aller Kurgane der ersten Verbreitungszone nach ihrer Größe

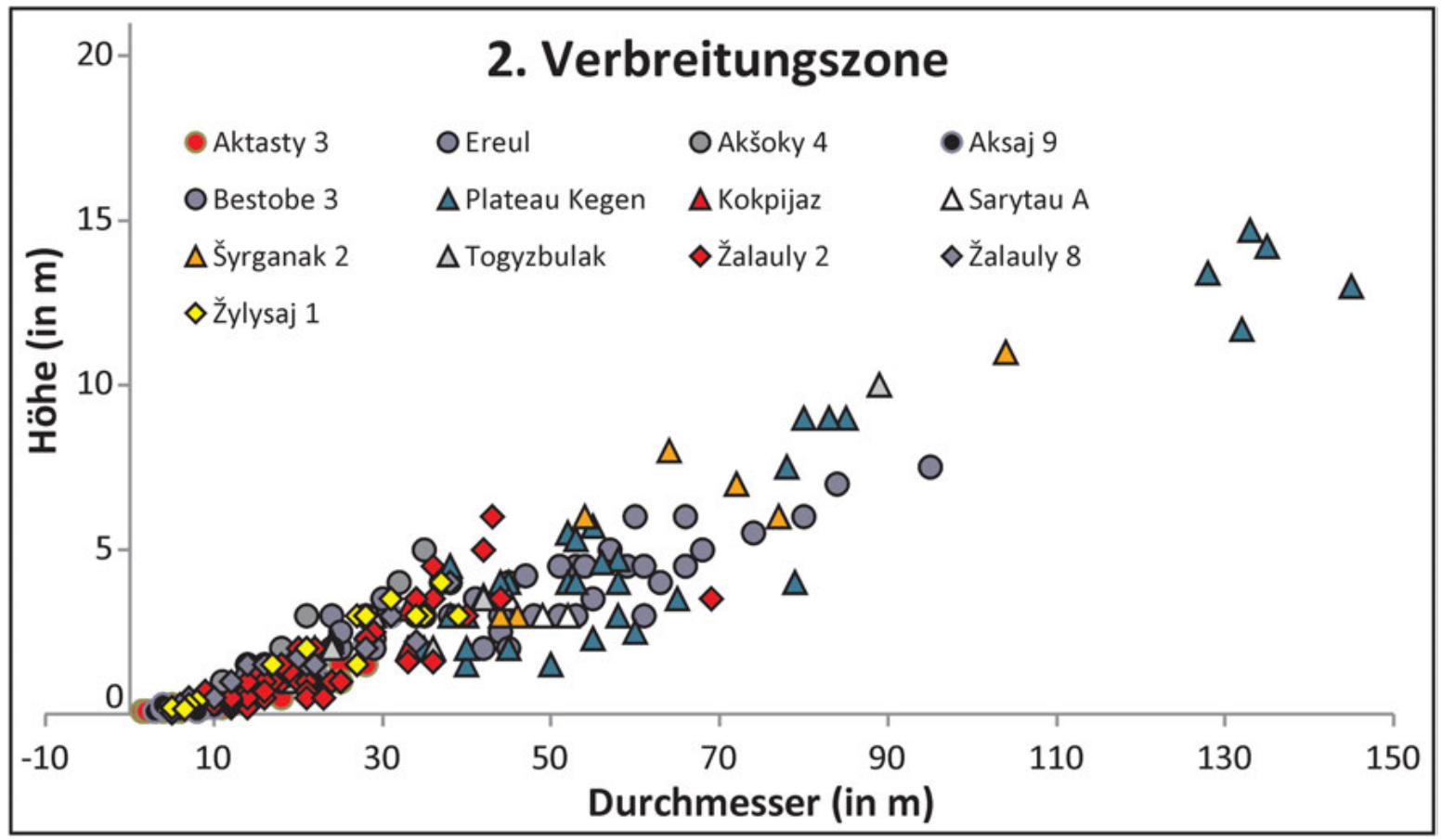

Abb. 93 | Gesamte statistische Verteilung aller Kurgane der zweiten Verbreitungszone nach ihrer Größe 

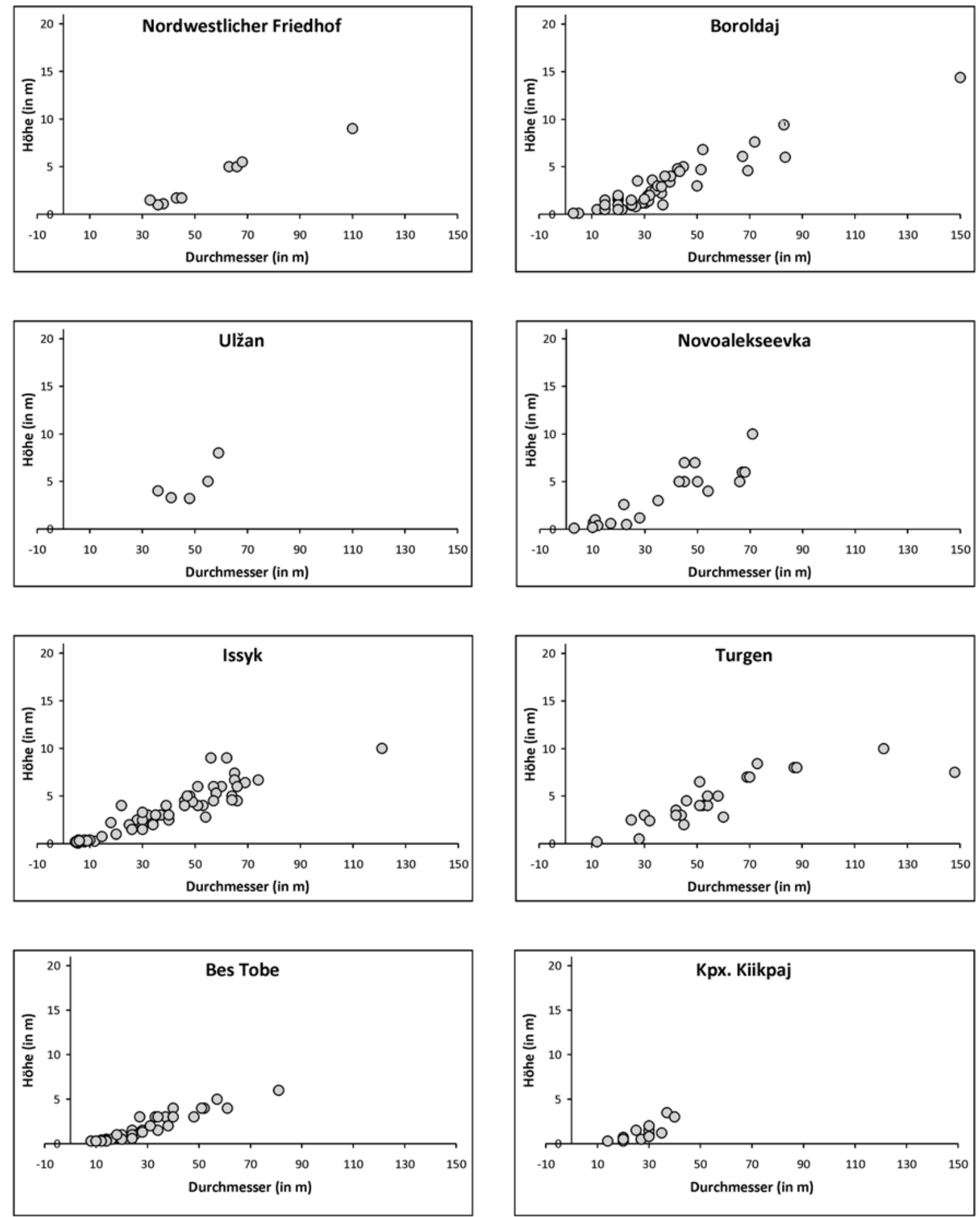

Abb. 94 | Statistische Verteilung der Kurgane nach ihrer Größe (Höhe und Durchmesser) auf einem bestimmten Gräberfeld. Fundstellen geographisch von Westen nach Osten geordnet 

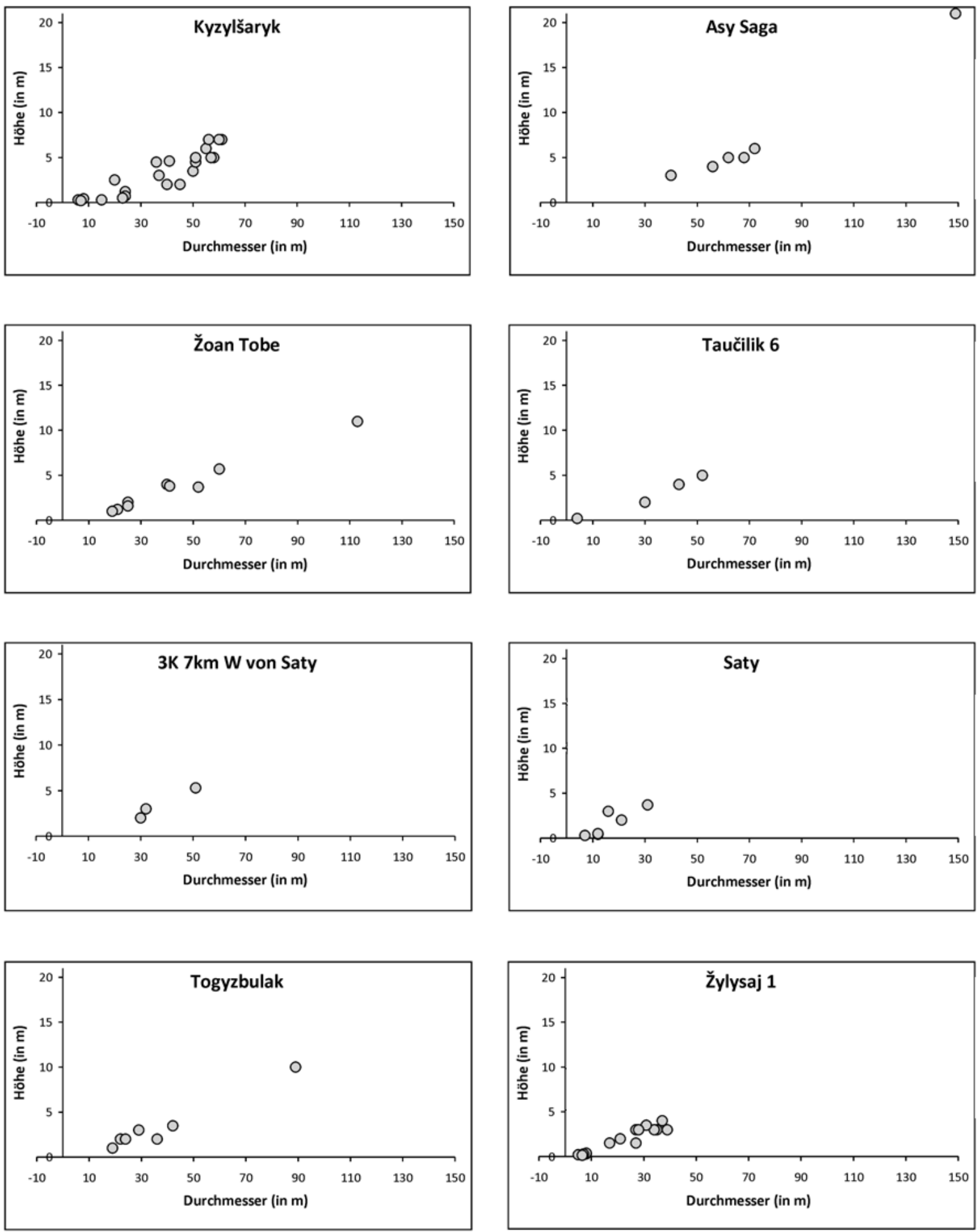

Abb. 94 | (Fortsetzung) 

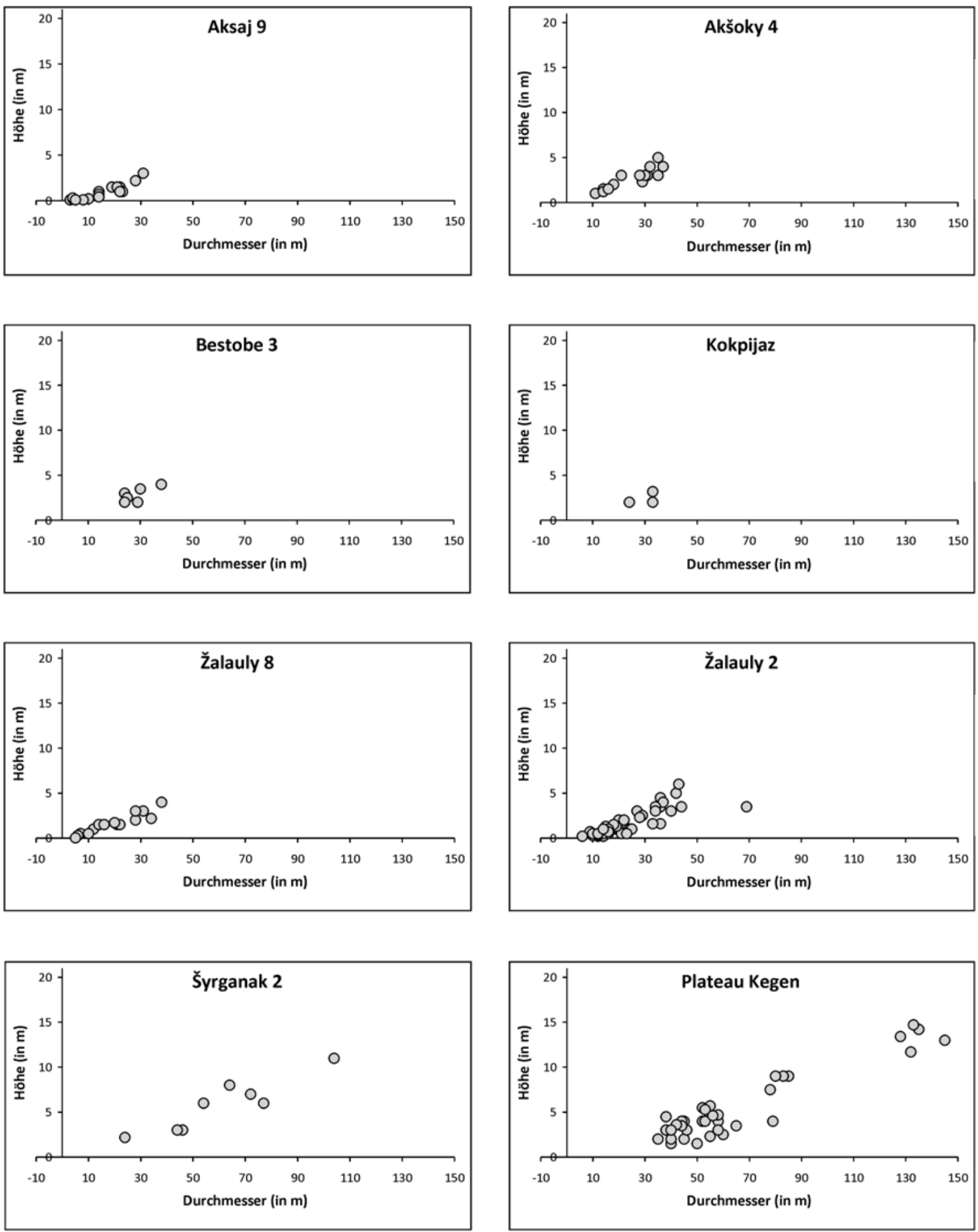

Abb. 94 | (Fortsetzung) 

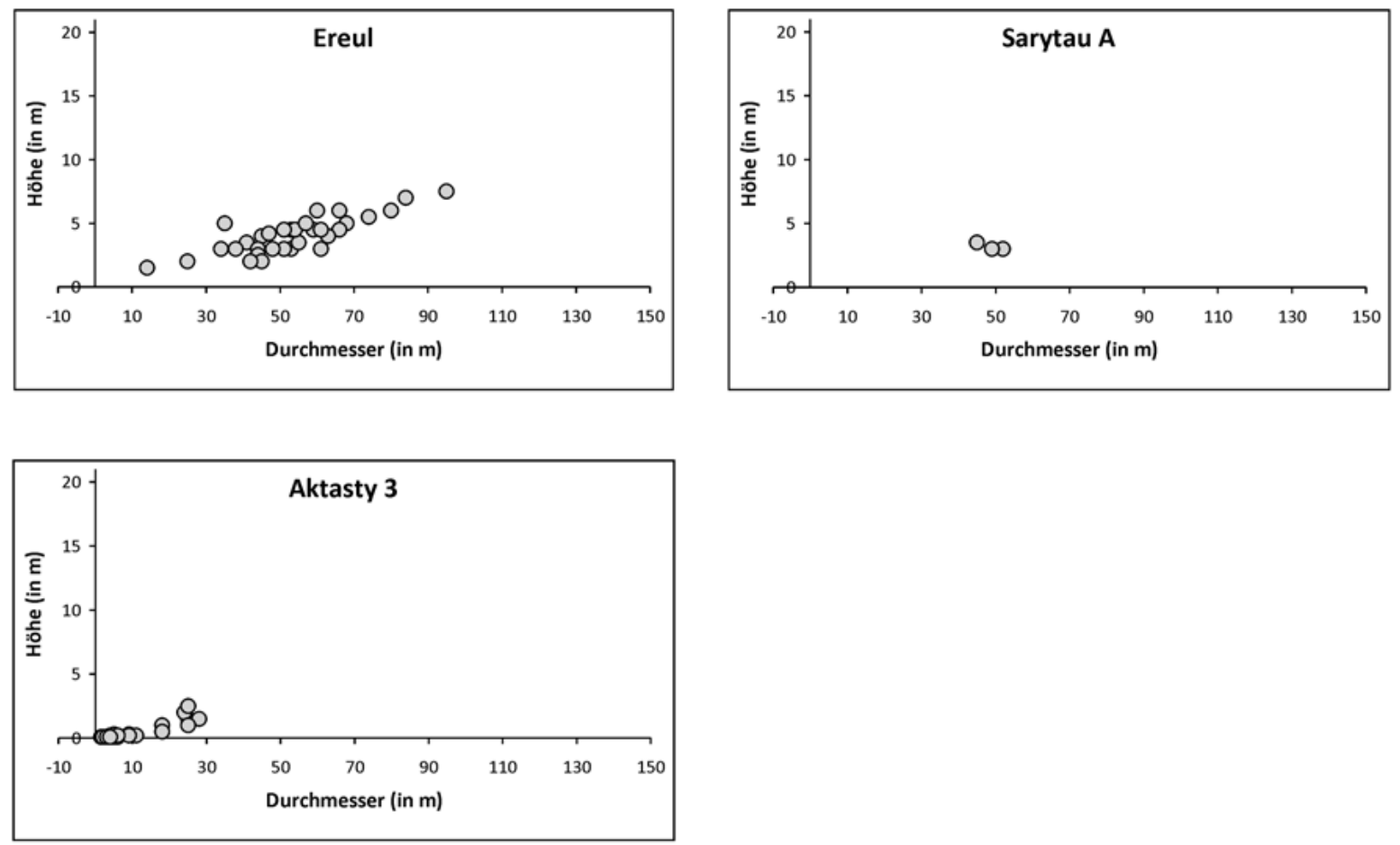

Abb. 94 | (Fortsetzung)

Zudem wurde bei fünf Gräberfeldern festgestellt, dass die Kurgane zwei Gruppen unterschiedlicher Größe bildeten495. Insgesamt stellten diese Nekropolen I8 \% aller untersuchten Gräberfelder dar. Vergleichbare Maße, die für mehrere Nekropolen galten, konnten jedoch nicht festgestellt werden. Gräberfelder mit Kurganen aus zwei Größengruppen wurden sowohl in der ersten als auch in der zweiten Verbreitungszone lokalisiert.

Bei acht Gräberfeldern, die über das gesamte Untersuchungsgebiet verteilt waren, bildeten die Kurgane je drei Gruppen unterschiedlicher Größen ${ }^{49}$. Der Anteil solcher Nekropolen macht $30 \%$ aus. Die Größe aller drei Gruppen scheint für jedes Gräberfeld individuell zu sein.

Zudem wurden vier Gruppen nach der Kurgangröße für Tumuli von verschiedenen Nekropolen zusammengefasst. Ähnlich wie bei den Gräberfeldern mit drei Gruppen von Kurgangrößen machte diese Gruppe ebenso 30\% der erforschten Nekropolen aus497. Solche Gräberfelder fanden sich in beiden Verbreitungszonen der sakischen Nekropolen mit Großkurganen des südöstlichen Siebenstromlandes. Die Größe dieser vier Gruppen war bei jedem einzelnen Gräberfeld verschieden.

In dieser Hinsicht scheint jede Nekropole individuell zu sein, wohingegen sowohl die Form der Kurgane als auch die Anordnung in Ketten überall das gleiche Prinzip zeigte. Jeweils ein Drittel aller untersuchten Gräberfelder bestand aus drei oder vier Gruppen von Kurgangrößen. Nur selten hatten

495 Nekropole des archäologischen Komplexes Kiikpaj, die Gräberfelder Taučilik-6, Žylysaj-I, Akšoky-4 und Žalauly-8.

496 Gräberfelder Kyzylšaryk, Asy Saga, Žoan Tobe, Togyzbulak, Aksaj-9, Žalauly-2, Ereul und Aktasty-3. lekseevka, Issyk, Turgen, Bes Tobe, Šyrganak-2 und die Nekropole auf dem Plateau Kegen. 
Abb. 95

Verhältnis der aus einfachen Kurganen bestehenden Gräberfelder und der Nekropolen mit verschiedenen Konstruktionen ihrer Peripherie

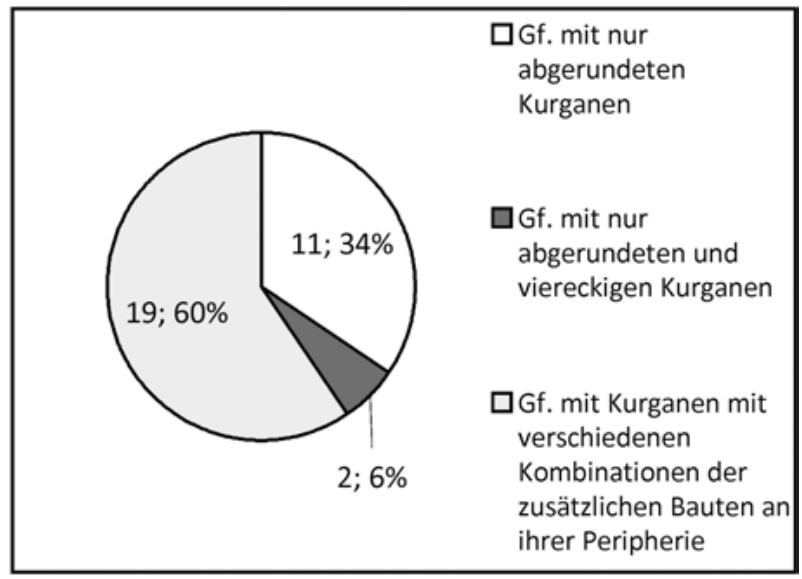

alle Kurgane eines Gräberfeldes ungefähr die gleiche Größe. In den wenigsten Fällen wurden zwei Größengruppen auf einem Gräberfeld beobachtet.

Knapp ein Drittel aller untersuchten Nekropolen (35\% - II Nekropolen; Abb. 95) bestanden ausschließlich aus gleichartigen Kurganen ohne jegliche Besonderheiten sowohl bezüglich der Kurgankonstruktion als auch der Peripherie. Auf zwei Gräberfeldern (6\%) befanden sich Kurgane von abgerundeter und viereckiger, pyramidenähnlicher Form, jedoch ohne weitere Konstruktionen in der Peripherie. Die überwiegende Zahl der Nekropolen (I9 Fundorte; 59\%) bestand aus Kurganen, die nicht nur eine unterschiedliche äußere Form zeigten, sondern auch verschiedene Kombinationen von zusätzlichen Konstruktionen in ihrer Peripherie aufwiesen. Außer um Kurgane mit einer abgerundeten oder viereckigen Form handelte es sich um Tumuli, die von Peripheriekonstruktionen, sog. Prozessionswegen, einem Kreisgraben, einem Steinkreis oder Sonderfällen flankiert werden.

Die Häufigkeitsanalyse zeigte (Abb. 96), dass sich auf 3I von 32 untersuchten sakischen Gräberfeldern (also in $97 \%$ der Fälle) abgerundete Kurgane befanden498. Jede dritte Nekropole bestand zum Teil aus viereckigen Kurganen (zehn Gräberfelder; 3I\%) oder aus Kurganen mit Peripheriekonstruktionen (zehn Gräberfelder; 31\%), bzw. aus Grabanlagen, die von einem sog. Prozessionsweg (zehn Gräberfelder; 31 \%) oder einem Steinkreis (elf Gräberfelder; $34 \%$ ) umgeben waren. Selten traten Gräberfelder auf, die in der Peripherie einiger Kurgane sog. Sonderfälle zeigten (sieben Nekropolen; 22 \%). Noch seltener kamen Nekropolen vor, auf denen sich Kurgane mit einem Kreisgraben befanden (sechs Gräberfelder; I9\%).

Auf I5 von I9 Nekropolen (Abb. 95) hatten die Kurgane nicht nur eine unterschiedliche äußere Form, sondern traten im Zusammenhang mit mehreren verschiedenen Kombinationen von Befunden im näheren Umfeld des Grabhügels auf. Auf den restlichen vier Gräberfeldern trat jeweils nur eine Art von weiterem Befund neben dem Kurgan auf und zwar bemerkenswerterweise immer bei abgerundeten (Tabelle 25). Es handelt sich um Gräberfelder mit Kurganen, die jeweils entweder von einem Kreisgraben oder von einem Steinkreis umgeben waren. Ferner kamen Gräberfelder zum Vorschein, auf denen die Kurgane ausschließlich Sonderfälle in ihrer Peripherie aufwiesen.

Wie die Tabelle 25 zeigt, traten jeweils in drei Nekropolen (je $9 \%$ aller untersuchten Gräberfelder) folgende Kombinationen auf: I. abgerundete Kurgane - Kurgane mit Peripheriekonstruktionen - Kur-

498 Noch ein Fundort bestand aus einzeln stehenden, viereckigen Großkurganen - ein Kurgan 5 km südwestlich des Dorfes Žalauly. 


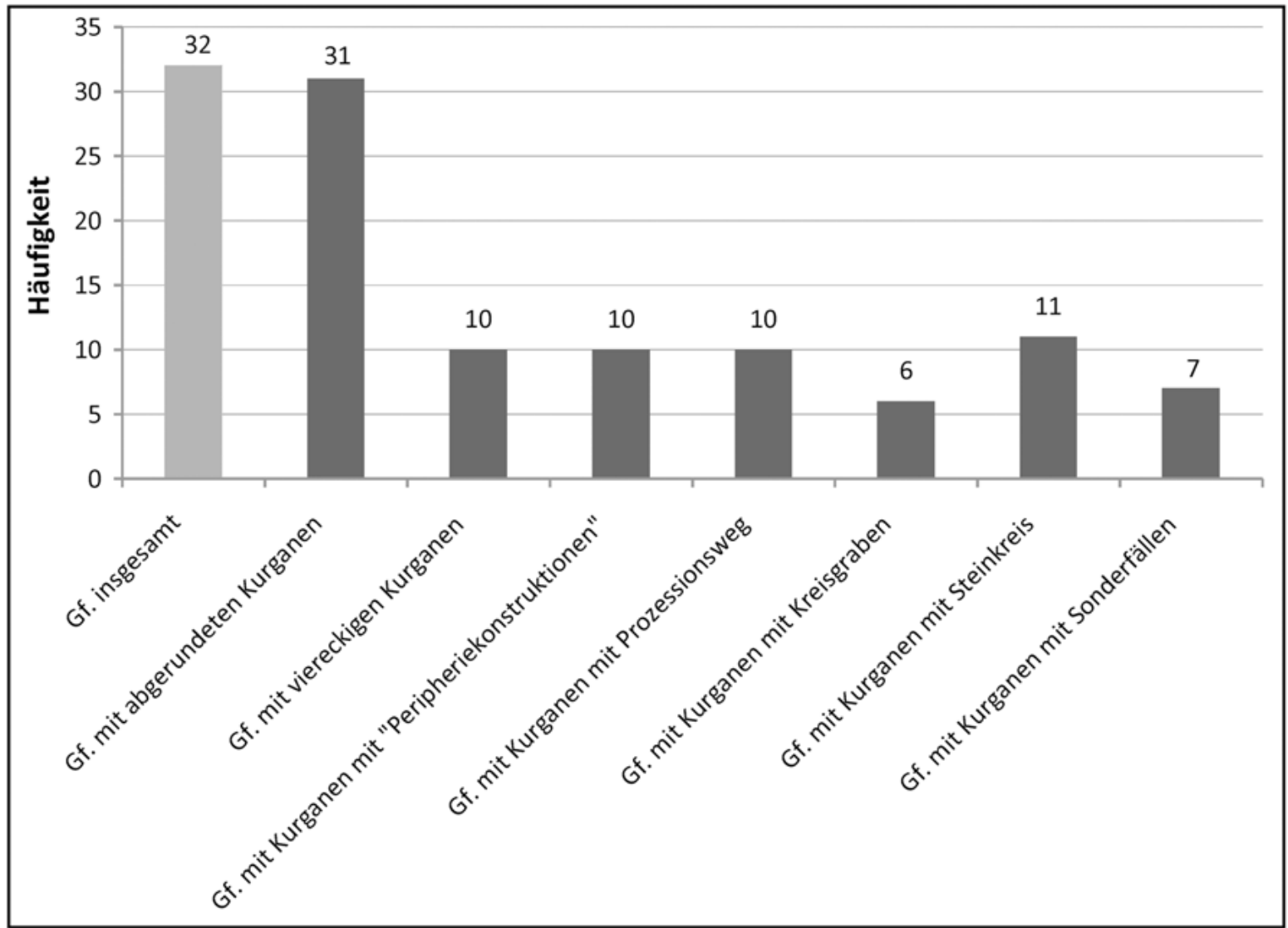

Abb. $96 \mid$

Häufigkeit des Vorkommens von Nekropolen mit Kurganen, die verschiedene Konstruktionen in ihrer Peripherie aufwiesen

gane mit einem sog. Prozessionsweg - Kurgane mit einem Steinkreis499 und 2. abgerundete Kurgane Kurgane mit einem Kreisgraben - Kurgane mit Sonderfällen in der Peripherie (Erdwälle bzw. doppelte Steinkreise sowie Plattformen und Baurampen)500.

Auf jeweils zwei Gräberfeldern (je 6\%) wurden folgende Kombinationen festgestellt: I. abgerundete Kurgane und Kurgane mit einem Steinkreis5or und 2. abgerundete und viereckige Kurgane - Kurgane mit Peripheriekonstruktionen - Kurgane mit einem sog. Prozessionsweg ${ }^{502}$. Beide Gräberfelder mit der Kombination „abgerundete und viereckige Kurgane - Kurgane mit Peripheriekonstruktionen Kurgane mit einem sog. Prozessionsweg“ lagen in der ersten Verbreitungszone, I7 km voneinander entfernt.

Alle anderen Kombinationen von Konstruktionen in der Peripherie kamen einmalig vor. Dabei wurden folgende Kombinationen festgestellt:

499 Gf. Aksaj-9 (Karte II Nr. 56), Gf. Aktasty-3 (Karte II Nr. 74) und Gf. Žalauly-2 (Karte II Nr. 62) - zweite Verbreitungszone.

500 Gf. Boroldaj (Karte II Nr. 2), Gf. auf dem Plateau Kegen (Karte II Nr. 68) und Gf. Žalauly-8 (Karte II Nr. 60) beide Verbreitungszonen.
50I Gf. Issyk (Karte II Nr. I4), Gf. Ereul (Karte in Nr. 69) und die Nekropole des archäologischen Komplexes Sarytau (Karte I5 Nr. 72) - beide Verbreitungszonen.

502 Gf. Kyzylšaryk (Karte II Nr. 28) und Gf. Žoan Tobe (Karte II Nr. 27). 
Tabelle 25 | Auflistung der architektonischen Merkmale der Kurgane (geographisch von Westen nach Osten und nach den Verbreitungszonen geordnet) /grau - erste Verbreitungszone, weiß - zweite Verbreitungszone; hellgrau - Raum zwischen den Zonen (Bartogaj- und Čilik-Tal)

\begin{tabular}{|c|c|c|c|c|c|c|c|c|}
\hline 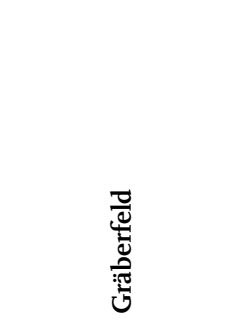 & 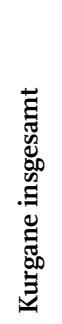 & 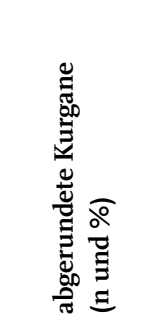 & 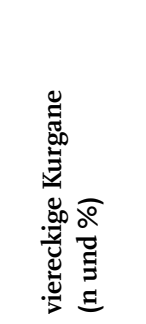 & 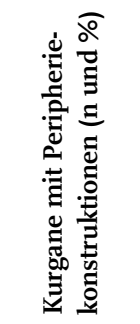 & 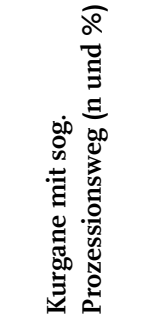 & 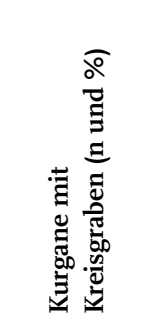 & 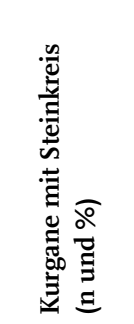 & 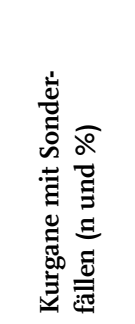 \\
\hline $\begin{array}{l}\text { Nordwestlicher } \\
\text { Friedhof }\end{array}$ & 9 & 9 (100\%) & & & & & & \\
\hline Boroldaj & 52 & 52 (100\%) & & 6 (II, $5 \%)$ & $2(3,8 \%)$ & I8 $(34,6 \%)$ & $3(5,8 \%)$ & $8(15,4 \%)$ \\
\hline Ulžan & 5 & 5 (100\%) & & & & & & I $(20 \%)$ \\
\hline Novoalekseevka & $2 I$ & I6 (76,2\%) & $5(23,8 \%)$ & & & & I $(4,8 \%)$ & \\
\hline Krasnyj Vostok & 2 & 2 (100\%) & & & & & & \\
\hline Issyk & 83 & 83 (100\%) & & & & & I (I,2\%) & \\
\hline Turgen & 36 & $35(97,2 \%)$ & I $(2,8 \%)$ & $2(5,6 \%)$ & $2(5,6 \%)$ & & $2(5,6 \%)$ & I $(2,8 \%)$ \\
\hline Bes Tobe & 47 & $46(97,9 \%)$ & I $(2, \mathrm{I} \%)$ & & & & & \\
\hline Komplex Kiikpaj & 15 & I5 (100\%) & & & & & & \\
\hline Kyzylšaryk & 37 & $36(97,3 \%)$ & I $(2,7 \%)$ & $3(8, \mathrm{I} \%)$ & $2(5,4 \%)$ & & & \\
\hline Asy Saga & 6 & $5(83,3 \%)$ & I (I6,7\%) & $2(33,3 \%)$ & $3(50 \%)$ & & & I (I6,7\%) \\
\hline Žoan Tobe & $3 I$ & $28(90,3 \%)$ & $3(9,7 \%)$ & I $(3,2 \%)$ & I $(3,2 \%)$ & & & \\
\hline Togyzbulak & 7 & 7 (100\%) & & & & I (I4,3\%) & & \\
\hline ŽYlysaj-1 & I8 & I7 $(94,4 \%)$ & I $(5,6 \%)$ & $\begin{array}{l}3 \\
(\mathrm{I} 6,7 \%)\end{array}$ & $7(38,9 \%)$ & & I $(5,6 \%)$ & \\
\hline Aksaj-9 & 30 & $30(100 \%)$ & & $2(6,7 \%)$ & I $(3,3 \%)$ & & 3 (10\%) & \\
\hline Akšoky-4 & I4 & I4 (I00\%) & & & & & & \\
\hline Bestobe-3 & 6 & 6 (100\%) & & & & & & \\
\hline Kokpijaz & 7 & 7 (I00\%) & & & & & & \\
\hline Žalauly-8 & I6 & I6 (I00\%) & & & & $6(37,5 \%)$ & & I $(6,3 \%)$ \\
\hline $\begin{array}{l}1 \mathrm{~K} 5 \mathrm{~km} S W \text { von } \\
\text { Žalauly }\end{array}$ & I & & I (I0०\%) & I (IO०\%) & & I (I00\%) & & \\
\hline Šyrganak-2 & 8 & 8 (100\%) & & & & & & \\
\hline Žalauly-2 & 68 & 58 (100\%) & & I $(\mathrm{I}, 5 \%)$ & I $(\mathrm{I}, 5 \%)$ & & $6(8,8 \%)$ & \\
\hline $\begin{array}{l}{ }_{2}^{2 K} 400 m \text { W von } \\
\text { Žalauly }\end{array}$ & 2 & 2 (I00\%) & & & & & & \\
\hline Plateau Kegen & 37 & 37 (100\%) & & & & I $(2,7 \%)$ & & I $(2,7 \%)$ \\
\hline Ereul & 35 & 35 (100\%) & & & & I $(2,9 \%)$ & I $(2,9 \%)$ & \\
\hline Sarytau „A“ & 3 & 3 (100\%) & & & & & & \\
\hline Komplex Sarytau & 103 & I03 (I00\%) & & & & & IO (9,7\%) & \\
\hline Aktasty-3 & 43 & 43 (100\%) & & I $(2,3 \%)$ & I $(2,3 \%)$ & & 6 (I4\%) & \\
\hline $\begin{array}{l}\text { Am Stausee } \\
\text { Bar-togaj }\end{array}$ & 39 & $38(97,4 \%)$ & I $(2,6 \%)$ & & $3(7,7 \%)$ & & $2(5, \mathrm{I} \%)$ & $3(7,7 \%)$ \\
\hline Taućilik-6 & 146 & I45 (99,3\%) & I $(0,7 \%)$ & & & & & \\
\hline${ }_{3} \mathrm{~K} 7 \mathrm{~km} \mathrm{~W}$ von Saty & 3 & 3 (100\%) & & & & & & \\
\hline Saty & 25 & 25 (100\%) & & & & & & \\
\hline Insgesamt: & 955 & 939 (98,3\%) & I6 (I,7\%) & $22(2,3 \%)$ & $23(2,4 \%)$ & $28(2,9 \%)$ & $27(2,8 \%)$ & I6 (I,7\%) \\
\hline
\end{tabular}


-abgerundete und viereckige Kurgane - Kurgane mit Peripheriekonstruktionen - Kurgane mit einem sog. Prozessionsweg - Kurgane mit einem Steinkreis - Kurgane mit Sonderfällen (Steinwall)503;

- $\quad$ abgerundete Kurgane - Kurgane mit Peripheriekonstruktionen - Kurgane mit einem sog. Prozessionsweg - Kurgane mit einem Kreisgraben - Kurgane mit einem Steinkreis - Kurgane mit Sonderfällen (zwei Erdwälle)504;

- $\quad$ abgerundete und viereckige Kurgane - Kurgane mit Peripheriekonstruktionen - Kurgane mit einem sog. Prozessionsweg - Kurgane mit Sonderfällen (Steinwall)505;

- $\quad$ abgerundete und viereckige Kurgane - Kurgane mit einem sog. Prozessionsweg - Kurgane mit einem Steinkreis - Kurgane mit Sonderfällen (steinkistenähnliche Konstruktionen)506;

- $\quad$ abgerundete und viereckige Kurgane - Kurgane mit Peripheriekonstruktionen - Kurgane mit einem sog. Prozessionsweg - Kurgane mit einem Steinkreis507;

- $\quad$ abgerundete und viereckige Kurgane - Kurgane mit einem Steinkreis508;

- $\quad$ viereckiger Kurgan - Kurgan mit Peripheriekonstruktionen - Kurgane mit einem Kreisgraben509;

- $\quad$ abgerundete Kurgane - Kurgane mit einem Kreisgraben - Kurgane mit einem Steinkreis5 ${ }^{\text {Io; }}$

- abgerundete Kurgane - Kurgane mit einem Kreisgraben5г;

- $\quad$ abgerundete Kurgane - Kurgane mit Sonderfällen (Erdwall) ${ }^{512}$.

Es bleibt zu vermerken, dass die sog. Prozessionswege um die Kurgane nie als einziges besonderes Element auf sakischen Gräberfelder auftraten. In Nekropolen, in denen sog. Prozessionswege vorkommen, wurden auch viereckige Kurgane, Kurgane mit Peripheriekonstruktionen, Kurgane mit Steinkreisen oder Kurgane mit Sonderformen festgestellt.

Eine regionalspezifische Kombination der oben erwähnten Merkmale konnte nicht festgestellt werden.

Zusammenfassend lässt sich sagen, dass die untersuchten Gräberfelder des südöstlichen Siebenstromlandes in Bezug auf die äußere Form der Kurgane, auf ihre Baumerkmale und auf die Anordnung der Kurgane in Ketten einem bestimmten Muster folgen. Jedoch scheint die Verteilung der Kurgane unterschiedlicher Größe in den einzelnen Nekropolen individuellen Regeln unterworfen zu sein. Ein Drittel aller untersuchten Gräberfelder bestand aus drei Gruppen von Kurgangrößen. Ein weiteres Drittel der Gräberfelder zeigte vier Größengruppen. Gräberfelder, die aus Kurganen von relativ gleicher Größe bestanden, kamen selten vor. Noch seltener wurden nur zwei Größengruppen von Kurganen beobachtet.

Auf jedem dritten Gräberfeld mit Kurganen der sakischen Elite (3I-34\%) im südöstlichen Siebenstromland standen viereckige Grabhügel, Kurgane mit Peripheriekonstruktionen, Kurgane mit sog. Prozessionswegen oder Kurgane mit einem Steinkreis.

Nur knapp ein Drittel der Gräberfelder wies identisch gebaute Kurgane ohne nennenswerte Unterschiede sowohl in der Kurgankonstruktion als auch der Peripherie auf. Es handelte sich hier um einfache abgerundete Kurgane ohne weitere Konstruktionen in ihrer Peripherie. Mehr als die Hälfte der untersuchten Nekropolen bestand aus Kurganen, die nicht nur eine unterschiedliche äußere Form aufwiesen, sondern auch verschiedene Kombinationen von zusätzlichen Konstruktionen in ihrer Pe-

503 Gf. Turgen (Karte in Nr. I9).

504 Gf. Boroldaj (Karte in Nr. 2).

505 Gf. Asy Saga (Karte II Nr. 29).

506 Gf. am Bartogaj-Stausee (Karte I3 Nr. 39).

507 Gf. Žylysaj-I (Karte II Nr. 50).

508 Gf. Novoalekseevka (Karte II Nr. Iо).
509 Ein Kurgan 5 km südwestlich vom Dorf Žalauly (Karte II Nr. 6I).

5I0 Gf. Ereul (Karte in Nr. 69).

5II Gf. Togyzbulak (Karte in Nr. 46).

5 I2 Gf. Ulžan (Karte in Nr. 3). 
ripherie zeigten. Allerdings kamen solche Kombinationen meist einzeln vor. Die Kombinationen, die sich zwei- bzw. dreimal wiederholten, konnten nicht als Spezifika eines bestimmten Gebietes definiert werden.

Diese Vielfalt der Konstruktionselemente, von denen abgerundete oder viereckige Kurgane umgeben waren, ist mit der Größe der Kurgane und nicht mit der Anzahl der Grabhügel in einer Nekropole zu erklären. Die unterschiedlichen Konstruktionselemente in der Peripherie markierten bestimmte sakrale Bereiche und stehen möglicherweise im Zusammenhang mit dem sozialen Status der bestatteten Individuen. Wozu jedes einzelne Konstruktionselement diente und welche Bedeutung es hatte, kann zum jetzigen Stand der Forschung nicht geklärt werden, da zur Beantwortung dieser Fragen eine vollständige Untersuchung der Anlagen erfolgen müsste.

Zur Geschichte der Kurgane der frühen Eisenzeit in der Region Transili-Alatau gibt es keine schriftlichen Überlieferungen. Über den nordpontischen Raum allerdings berichteten antike Autoren, wie z.B. Herodot (Parzinger 2004, 7-18; Mozolevskij/Polin 2005, I7-I8). Einerseits, ähneln die Kurgane äußerlich den skythischen Heiligtümern (Herodot [4-62], 30I-302), andererseits stellten die Nekropolen der Elite Orte des kollektiven Gedächtnisses dar, die für Skythen / Saken eine bedeutende Rolle spielten (Herodot [4-7I, 4-I27] 305, 327-328). Im Bewusstsein der früheisenzeitlichen Reiternomaden waren die Nekropolen nicht nur Bestattungsorte, sondern auch zentrale Sakralbereiche (Gass 20IIb, 223).

\subsection{Der Bezug der Gräberfelder zum Gelände}

Im südöstlichen Siebenstromland sind zwei Verbreitungszonen mit Gräberfeldern der sakischen Elite zu unterscheiden (siehe Anfang des Kap. 5. Sakische Nekropolen mit Fürstenkurganen als Zentralorte der Nomaden). Drei Gräberfelder befinden sich zwischen diesen beiden Zonen, nämlich im Čilik-Tal (Karte II).

Die erste Verbreitungszone wurde an der Nordseite des Transili-Alatau, der den nördlichen TienShan bildet, lokalisiert. Die Gräberfelder wurden am Rande des nördlichen Lösshügellandes des Transili-Alatau angelegt. Sie befanden sich bereits auf dem Schwemmfächer (800-900 $\mathrm{m}$ ü NN) und sind im Durchschnitt I,5-3,0 km vom Lösshügelland entfernt (Karte I8). Das gesamte Gebiet befindet sich in der Zone des Kontinentalklimas (Karte 5) und weist Tschernosem- und Kastanozem-Böden auf (siehe Kap. 2. Geographische Lage und klimatische Verhältnisse des Untersuchungsgebietes).

Die Gräberfelder kommen stets in der Nähe von meridianal fließenden Gewässern vor (Tabelle 26) und zwar auf deren zweiter bzw. dritter Flussuferterrasse und wiederholten meist den Verlauf des Flusses, sogar im Falle von Flussbiegungen. Alle Flüsse der ersten Verbreitungszone entspringen im südlich gelegenen Gebirge Transili-Alatau und fließen nach Norden, bis sie als linke südliche Nebenflüsse in den Fluss Ili münden. Der Abstand zwischen Gräberfeld und Ufer beträgt o,2 km553 bis 8 km514. Die durchschnittliche Entfernung einer Nekropole vom Fluss misst I,I km. Gliedert man die Entfernungen der Nekropolen vom Ufer in drei Kategorien (unter I km, I-3 km und über $3 \mathrm{~km}$ ) ${ }^{55}$, ergibt sich Folgen-

5I3 Gf. Ulžan.

5I4 Gf. Žoan Tobe.
5I5 Da die Häufigkeitsanalyse keine eindeutigen Gruppen erbrachte, wurden die Entfernungskategorien künstlich festgelegt. 
Tabelle 26 | Verhältnis der sakischen Gräberfelder der ersten Verbreitungszone zu den nächstgelegenen fließenden Gewässern (Fundorte geographisch von Westen nach Osten aufgeführt)

\begin{tabular}{|l|l|l|l|l|}
\hline Gräberfeld & Gewässer & Uferseite & Ufer & Entfernung (km) \\
\hline Nordwestlicher Friedhof & Fluss Boroldaj & linke & W & 2,5 \\
\hline Boroldaj & $\begin{array}{l}\text { zwischen Flüssen Boroldaj / Bol'šaja } \\
\text { Almatinka }\end{array}$ & linke / rechte & W / O & I,3/ O,5 \\
\hline Ulžan & Fluss Bol'šaja Almatinka & rechte & O & 0,2 \\
\hline Novoalekseevka & Fluss Talgar & linke & W & 2,5 \\
\hline Kurgangruppe Krasnyj Vostok & Bergfluss & rechte & O & 0,2 \\
\hline Issyk & Fluss Esik & linke & W & I,5 \\
\hline Turgen & Fluss Turgen & linke / rechte & W / O & $0,6 / 0,8$ \\
\hline Bes Tobe & Fluss Kiikbaj & linke & W & 2,0 \\
\hline Kiikpaj & Fluss Kiikbaj & linke & W & 0,5 \\
\hline Kyzylšaryk & Fluss Asysr6 & linke & W & 7,0 \\
\hline Asy Saga & Fluss Asy & linke & W & 2,8 \\
\hline Žoan Tobe & Fluss Kuržilik & rechte & O & 8,0 \\
\hline
\end{tabular}

des: In der ersten Verbreitungszone befanden sich fünf von zwölf früheisenzeitlichen Nekropolen mit Kurganen der sakischen Elite (also $42 \%$ aller untersuchten Gräberfelder erster Verbreitungszone), die weniger als einen Kilometer vom nahe gelegenen Fluss entfernt waren. Weitere fünf Nekropolen (42\%) lagen zwischen I bis zu $3 \mathrm{~km}$ vom nächstgelegenen Gewässer entfernt. Einen Abstand über $3 \mathrm{~km}$ wiesen nur zwei Gräberfelder auf.

Offensichtlich spielte die Uferseite eine Rolle, allerdings nicht immer (Abb. 97). Gegenüber sieben Gräberfeldern (59\%), die am linken, westlichen Ufer errichtet wurden, treten drei Nekropolen (25\%) auf dem rechten, östlichen auf. Das Gräberfeld Boroldaj liegt auf einem Plateau, das wie eine „Insel“ an beiden Seiten von Flüssen flankiert wurde. Die Nekropole Turgen erstreckt sich auf beiden Ufern des gleichnamigen Flusses.

In der ersten Verbreitungszone konnte folglich die Lage der Nekropolen auf einem linken, westlichen Flussufer als dominant bezeichnet werden. Es gibt jedoch auch Beispiele, wo die Gräberfelder auch auf der rechten, östlichen Uferseite lokalisiert wurden. Als Einzelfälle gelten Gräberfelder, die sich zwischen zwei Flüssen bzw. auf beiden Uferseiten befanden.

Die zweite Verbreitungszone der früheisenzeitlichen Nekropolen mit großen Fürstenkurganen wurde auf dem Hochplateau Kegen (I853-2055 m ü NN) und jenseits der Kuluktau-Gebirge (Nordseite; I428-I460 $\mathrm{m}$ ü NN) festgestellt. Die Gräberfelder befanden sich im mittleren Bereich des Plateaus und auf dessen Nordhälfte sowie an der Nordseite des Kuluktau-Gebirges. Auf dem Plateau Kegen lagen die untersuchten Nekropolen entweder im Flachland, entlang der das Plateau durchfließenden Flüsse, oder auf den Riedelkanten der südlichen Ausläufer des Kuluktau-Gebirges. Die Lagemerkmale der Gräberfelder an der Nordseite des Kuluktau-Gebirges ähnelten denen der untersuchten Nekropolen der ersten Verbreitungszone. Der einzige Unterschied bestand darin, dass die Gräberfelder I,O-2,5 km von 
Abb. 97 | Verhältnis der Lage der sakischen Gräberfelder der ersten Verbreitungszone bezüglich des Flussverlaufs und der Himmelsrichtungen

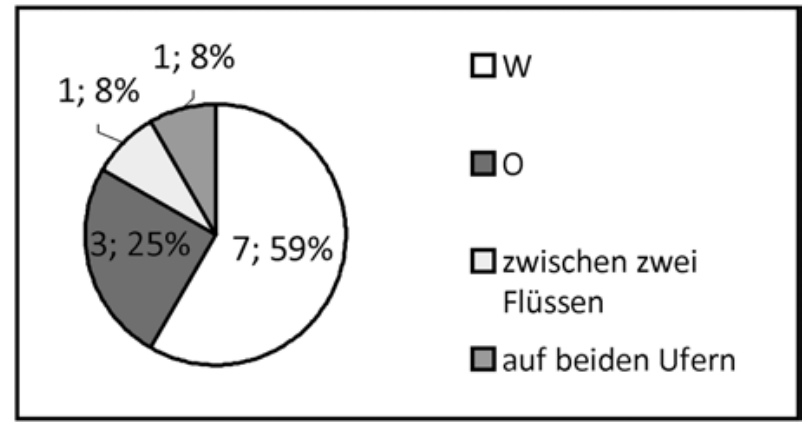

dem Lösshügelland entfernt waren. Eine weitere, kleine Konzentration von Nekropolen5r7 befand sich in den Bergtälern des Kuluktau-Gebirges (I903-I997 m ü NN). Die zweite Verbreitungszone befindet sich im Bereich des nivalen Klimas (Karte 5) und weist Tschernosem- und Kastanozem-Böden auf (siehe Kap. 2. Geographische Lage und klimatische Verhältnisse des Untersuchungsgebietes).

Fast alle Gräberfelder liegen in der Nähe von fließenden Gewässern, die entweder von Süden nach Norden oder von Osten nach Westen fließen (Tabelle 27). In einigen Fällen wurden die Gräberfelder auf Riedelkanten in Flussnähe errichtet. Alle Flüsse des Plateaus Kegen münden in den gleichnamigen, größten Fluss des Plateaus, der das Plateau zuerst von Südosten nach Nordwesten und am Nordrand seine Fließrichtung ändernd von Osten nach Westen durchfließt. Nachdem der Kegen das Plateau in seiner nordöstlichen Ecke verlässt, fließt er durch den Čaryn Canyon nach Nordnordost und wird hier als Fluss Šaryn bezeichnet. Folglich münden die Flüsse und Bäche an der Nordseite des Kuluktau-Gebirges in den Šaryn.

Wie die Tabelle 27 zeigt, befinden sich neun Gräberfelder (60\% von I5 Nekropolen der zweiten Verbreitungszone) auf der Ebene. Sechs weitere Nekropolen wurden auf den Riedelkanten errichtet. Ein Bezug zu fließenden Gewässern konnte für I4 Gräberfelder festgestellt werden. Eine Ausnahme stellt die Nekropole Bestobe-3 dar, die in einem Bergtal des Kuluktau-Gebirges liegt.

Von den I4 Gräberfeldern befinden sich fünf (33\% aller Nekropolen der zweiten Verbreitungszone) zwischen Flüssen bzw. Bächen. Sechs weitere Gräberfelder (40\%) wurden auf dem rechten Flussufer errichtet. Drei Nekropolen (20\%) wurden auf dem linken Flussufer lokalisiert.

Der Abstand zwischen der Nekropole und dem Ufer schwankte zwischen o,I km518 und 3,2 km519. Die durchschnittliche Entfernung zwischen Fluss und Gräberfeld betrug I,3 km. Gliedert man die Nekropolen nach der Entfernung zum Ufer eines Flusses, wie es bereits für die erste Verbreitungszone erfolgte, in drei Kategorien (unter I km, I-3 km und über $3 \mathrm{~km}$ ) 520 , erhält man folgendes Ergebnis: Fünf Gräberfelder (33\%) liegen weniger als einen Kilometer entfernt, acht Nekropolen (53\%) zwischen I km und $3 \mathrm{~km}$. Das Gräberfeld Ereul befindet sich über $3 \mathrm{~km}$ vom Fluss Karkara weg, und zwar von dessen Hauptarm. Das Gräberfeld Bestobe-3 steht nicht im Bezug zu fließenden Gewässern.

Falls ein Gräberfeld nicht zwischen Flüssen bzw. Bächen platziert worden war, spielte, im Unterschied zur Uferseite, die Orientierung fast keine Rolle. Wie die Abbildung 98 zeigt, wurden die Nekropolen meist entweder auf dem westlichen oder nördlichen Ufer lokalisiert (jeweils drei Gräberfelder; $20 \%$ aller untersuchten Nekropolen der zweiten Verbreitungszone). In einigen Fällen traten sie auch

5 I7 Gf. Akšoky-4 und Gf. Bestobe-3.

5I8 Gf. Žalauly-8.

5I9 Gf. Ereul.
520 Da die Häufigkeitsanalyse keine sichtbaren Gruppen zum Ergebnis hatte, wurden die Entfernungskategorien künstlich festgelegt. 
Tabelle 27 | Verhältnis der sakischen Gräberfelder der zweiten Verbreitungszone zu den fließenden Gewässern (Fundorte geographisch von Westen nach Osten aufgeführt)

\begin{tabular}{|c|c|c|c|c|c|}
\hline Gräberfeld & Riedelkannte & Gewässer & Uferseite & Ufer & Entfernung (km) \\
\hline Togyzbulak & & Fluss Šet-Merke & linke & $\mathrm{W}$ & 2,3 \\
\hline Žylysaj-I & & $\begin{array}{l}\text { zwischen ausgetrockne- } \\
\text { ten Bachbetten }\end{array}$ & rechte / linke & $\mathrm{O} / \mathrm{W}$ & $0,6 / 0,6$ \\
\hline Aksaj-9 $9^{521}$ & ja & $\begin{array}{l}\text { zwischen periodisch-sai- } \\
\text { sonalen Bächen }\end{array}$ & rechte / linke & $\mathrm{O} / \mathrm{W}$ & $0,2 / 0,4$ \\
\hline Akšoky-4 & ja & Fluss Šybyšy & rechte & $\mathrm{W}$ & $\mathrm{I}, 4$ \\
\hline Bestobe- 3 & & keine - liegt im Bergtal & $-1-$ & $-1-$ & $-1-$ \\
\hline Kokpijaz & & Fluss Žinsajbulak & linke & $\mathrm{W}$ & 0,9 \\
\hline Žalauly-8 & ja & Fluss Kegen & rechte & $\mathrm{N}$ & $\mathrm{O}, \mathrm{I}$ \\
\hline $\begin{array}{l}\text { Ein Kurgan } 5 \text { km süd- } \\
\text { westlich vom Dorf Ža- } \\
\text { lauly }\end{array}$ & ja & Fluss Kegen & rechte & NW & $\mathrm{I}, \mathrm{O}$ \\
\hline Šyrganak-2 & & $\begin{array}{l}\text { zwischen den Flüssen } \\
\text { Žarganak und Karkara }\end{array}$ & rechte / linke & $\mathrm{O} / \mathrm{W}$ & $\mathrm{I}, 4 / 3, \mathrm{O}$ \\
\hline Žalauly-2 & ja & Fluss Kegen & rechte & $\mathrm{N}$ & 0,4 \\
\hline $\begin{array}{l}\text { Zwei Kurgane } 400 \text { m } \\
\text { westlich vom Dorf Ža- } \\
\text { lauly }\end{array}$ & ja & Fluss Kegen & rechte & $\mathrm{N}$ & $\mathrm{I}, 2$ \\
\hline $\begin{array}{l}\text { Gf. auf dem Plateau Ke- } \\
\text { gen }\end{array}$ & & $\begin{array}{l}\text { an beiden Ufern des } \\
\text { Flusses Karkara und am } \\
\text { Fluss Kegen }\end{array}$ & linke / rechte / linke & $\mathrm{W} / \mathrm{O} / \mathrm{W}$ & $\mathrm{I}, 7 / 2,6 / 2,4$ \\
\hline Ereul & & $\begin{array}{l}\text { zwischen kleinen Neben- } \\
\text { flüssen des Flusses Kar- } \\
\text { kara; Fluss Karkara }\end{array}$ & rechte $^{522}$ & $\mathrm{O} 523$ & $3,2^{524}$ \\
\hline Sarytau „A“ & & Fluss Kegen & rechte & $\mathrm{O}$ & 2,2 \\
\hline Aktasty-3 & & Fluss Aktasty & linke & $\mathrm{O}$ & 0,3 \\
\hline
\end{tabular}

auf dem östlichen (zwei Gräberfelder; I3\%) oder auf dem nordwestlichen (eine Nekropole; $7 \%$ ) Ufer auf.

Drei Gräberfelder525, die im schmalen Čilik-Tal (I602-I486 m ü NN), an der Nordseite des KungejAlatau liegen und sich zwischen den beiden Verbreitungszonen befinden, wurden an beiden Ufern des Flusses Čilik errichtet. Der Čilik fließt hier von Westen nach Osten und im Tal herrscht nivales Klima (Karte 5). Zwei Gräberfelder ${ }^{26} 6$ wurden auf dem linken nördlichen und ein weiteres ${ }^{527}$ auf dem rechten südlichen Ufer lokalisiert. Der Abstand zwischen beiden Nekropolen am nördlichen Ufer beträgt ca. Io km. Drei Kurgane $7 \mathrm{~km}$ westlich des Dorfes Saty, die sich am südlichen Ufer des Čilik befinden, liegen relativ mittig zwischen beiden Gräberfeldern des nördlichen Ufers, ca. $5 \mathrm{~km}$ von beiden Gräberfeldern entfernt.

52I Die Gräberfelder, die sich nicht auf dem Plateau Kegen befinden, Tugyzbulak, Žylysaj-I und Aksaj-9, sind als erste nach ihrer geographischen Lage von Westen nach Osten aufgeführt und anschließend diejenigen auf dem Plateau von Westen nach Osten.

522 Im Bezug zum Karkara-Fluss.
523 Siehe Anm. 520.

524 Siehe Anm. 520.

525 Gf. Taucilik-6 und Gf. Saty sowie drei Kurgane 7 km westlich vom Dorf Saty (Karte II Nr. 42, 45 und 43).

526 Gf.Taučilik-6 und Gf. Saty.

527 Drei Kurgane, $7 \mathrm{~km}$ westlich vom Dorf Saty. 
Abb. 98 | Verhältnis der Lage der sakischen Gräberfelder zweiter Verbreitungszone bezüglich des Flussverlaufs und der Himmelsrichtungen

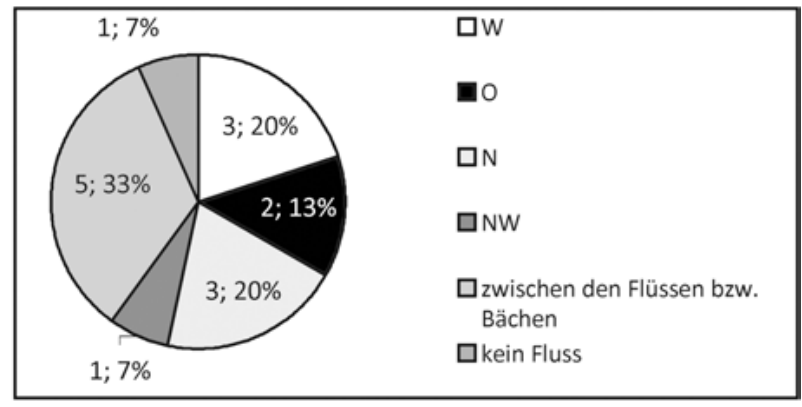

Zusammenfassend kann festgestellt werden, dass die Gräberfelder der zweiten Verbreitungszone am häufigsten zwischen zwei fließenden Gewässern errichtet wurden oder sich überwiegend auf dem rechten nördlichen, auf dem rechten nordöstlichen oder auf dem rechten östlichen Ufer des Flusses bzw. Baches befanden. Im Unterschied zu den Gräberfeldern in der ersten Verbreitungszone, wo die Gräberfelder besonders häufig am linken westlichen Ufer angelegt worden waren, spielte in der zweiten Verbreitungszone die Himmelsrichtung offensichtlich nicht so eine wichtige Rolle, da die Flüsse auf dem Plateau Kegen relativ oft ihren Verlauf ändern. Die Lage des Gräberfeldes innerhalb der Landschaftseinheiten spielte offensichtlich eine wichtige Rolle. Die Gräberfelder der zweiten Verbreitungszone wurden entweder am rechten Ufer oder zwischen den fließenden Gewässern errichtet.

Im Gegensatz zur eben beschriebenen Situation fließen die Gewässer in der ersten Verbreitungszone ausnahmslos meridianal von Süden nach Norden. Die Gräberfelder mit Kurganen der sakischen Elite befanden sich hier meist auf dem linken westlichen Ufer. Die Gewässer selbst stellten möglicherweise eine natürliche Grenze zwischen den sakralen Bereichen der Nekropolen dar.

Fazit:

Fasst man die Ergebnisse zu den Untersuchungen der Nekropolen mit Kurganen der sakischen Elite im südöstlichen Siebenstromland zusammen, lassen sich folgende Aussagen treffen:

- Im Arbeitsgebiet konnte eine hohe Konzentration früheisenzeitlicher Kurgane festgestellt werden, die sich auf einfache Gräberfelder, einzeln stehende Kurgane und auf Nekropolen, die in direkter Verbindung zu einer Siedlungsstelle stehen, sog. „archäologische Komplexe“, verteilen.

- Die untersuchten Gräberfelder konzentrierten sich in zwei Bereichen, die ähnliche Landschaftsbedingungen aufweisen.

- $\quad$ Die erste Verbreitungszone umfasst die nördliche Seite des Tien-Shan, den Übergang des Lösshügellandes in den Schwemmfächer. Die Nekropolen liegen auf den Schwemmfächern, I,5-3,0 km vom Lösshügelland entfernt.

- $\quad$ Die zweite Verbreitungszone erstreckt sich über das Hochplateau Kegen bis zur Nordseite des Kuluktau-Gebirges.

- Das Čilik-Tal an der Nordseite des Kungej-Alatau bildet einen Zwischenraum zwischen den zwei Verbreitungszonen. Hier befinden sich einige Gräberfelder.

- Die Größe der Kurgane auf einem Gräberfeld variiert. Sie reicht von einigen wenigen Metern bis zu über hundert Meter im Durchmesser und von einigen Zentimetern bis zu über zehn Meter in der Höhe.

- Als Kurgane der Elite wurden die Anlagen angesprochen, die einen Durchmesser von über $30 \mathrm{~m}$ und eine Höhe von über $2 \mathrm{~m}$ aufweisen. 
- Die Kurgane der früheisenzeitlichen sakischen Elite wurden in drei Größenkategorien unterteilt: a) sehr große Kurgane (Höhe über $8 \mathrm{~m}$ ), b) größere Kurgane $(4-8 \mathrm{~m})$ und c) große Kurgane $(2-4 \mathrm{~m})$.

- Die Größenkategorien sind relativ gleichmäßig über beide Verbreitungszonen verteilt. Allerdings ist der Anteil der größeren Bauten auf einer Nekropole in der ersten Verbreitungszone wesentlich höher.

- Die einzelnen Kurgane sind zu Ketten aneinandergereiht. Ein Gräberfeld kann von einer bis zu elf Ketten aufweisen. Die Ketten sind meist nordsüdlich ausgerichtet und befinden sich an den Flussuferterrassen oder auf den Riedelkanten, abhängig vom Landschaftsrelief des Untersuchungsgebietes.

- Innerhalb einer Kette sowie innerhalb einer Nekropole wurden die größten Kurgane jeweils in der Nordhälfte lokalisiert. In einigen Fällen jedoch wurden Konzentrationen von sehr großen Kurganen im mittleren Bereich oder in der Südhälfte der Kette und zugleich des Gräberfeldes festgestellt.

- Außerhalb der Ketten wurden außerdem einzeln stehende Großkurgane festgestellt. Ihre Lage in den Nekropolen variierte.

- Die meisten sakischen Großkurgane stellten abgerundete, plattformähnliche Konstruktionen mit einer abgeflachten Kuppe dar. Im Gegensatz zu den Kleinkurganen wiesen 86\% der Großkurgane drei steile und einen sanften Abhang auf. Als die häufigste Ausrichtung des sanften Abhanges konnte die Südseite festgestellt werden. Es trat aber auch die westliche, östliche, südöstliche, südwestliche und nordwestliche Ausrichtung auf. Die unterschiedlichen Ausrichtungen des sanften Abhanges kamen nur im Bereich der ersten Verbreitungszone vor. In der zweiten Verbreitungszone wurden die Kurgane nur in südlicher Richtung, mit kleinen Abweichungen, orientiert.

- Da innerhalb einiger Ketten verschieden orientierte Kurgane auftraten, kann man davon ausgehen, dass die Ausrichtung bereits ganz bewusst bei der Errichtung festgelegt und nicht erst später durch Verwitterungsprozesse beeinflusst wurde.

- Die asymmetrische Form der Kurgane im südöstlichen Siebenstromland findet mehrere Parallelen bei den Kurganen des Steppen- und Waldsteppengürtels Sibiriens. Weitere Analogien sind mit den Grabhügeln der europäischen Skythen zu nennen, die zudem durch die schriftlichen Quellen, besonders die Herodots, bekannt sind. Herodot zufolge wurden für die skythischen Könige riesige Kurgane errichtet. Zur äußeren Form der Grabanlagen äußerte sich der Historiker allerdings kaum. Die Heiligtümer des Ares wurden dagegen sehr genau beschrieben. In dieser Beschreibung finden sich sehr viele Ähnlichkeiten mit der äußeren Form eines Kurgans. Diese erstaunliche Übereinstimmung der sakischen Elitenkurgane mit den skythischen Heiligtümern des Ares lässt eine gewisse Intension vermuten.

- Jede dritte untersuchte Nekropole wies ein bis drei viereckige, pyramidenähnliche Kurgane mit abgeflachter Kuppe auf, die meist in der Südhälfte des Gräberfeldes standen. Solche Kurgane traten, außer bei den sehr großen Kurganen, in allen vorkommenden Größenkategorien auf und besaßen drei steile und einen sanften Abhang. In einer Kette befand sich nur je ein pyramidenähnlicher Kurgan, wobei insgesamt auf einem Gräberfeld mehrere viereckige Kurgane vorkamen. Innerhalb einer Kette konnten solche Kurgane sowohl in der Nord- als auch in der Südhälfte lokalisiert werden.

- Die Bedeutung der viereckigen, pyramidenähnlichen Kurgane kann momentan nicht geklärt werden. Die wenigen Versuche zur Erforschung solcher Kurgane lieferten aufgrund der völligen Beraubung und des daraus resultierenden schlechten Zustandes der Gräber kaum Informationen. 
Dementsprechend ist die Problematik der pyramidenähnlichen Kurgane des südöstlichen Siebenstromlandes als Desiderat der Forschung zu betrachten.

- Alle untersuchten Gräberfelder folgen bezüglich der äußeren Form der Kurgane, der Bauweise und der Anordnung in Ketten einem Muster. Allerdings scheint jede Nekropole hinsichtlich Konzentration und Verteilung der Kurgane unterschiedlicher Größe ein eigenes Prinzip zu haben.

- $\quad$ Trotz der Zerstörung der Peripherie mehrerer Kurgane konnten in diesem Randbereich zusätzliche Konstruktionen, wie Peripheriekonstruktionen, sog. Prozessionswege, Kreisgräben, Steinkreise oder sog. Sonderfälle, festgestellt werden. Kurgane mit solchen zusätzlichen Befunden kamen im gesamten Untersuchungsgebiet zum Vorschein.

- Peripheriekonstruktionen wurden überwiegend durch Kleinkurgane repräsentiert. Zudem kamen rechteckige, kistenähnliche Steinkonstruktionen oder viereckige bis abgerundete Eintiefungen vor. Diese Objekte lagen jeweils südlich oder östlich des Großkurgans.

- In jeder dritten Nekropole konnten solche Kurgane mit Peripheriekonstruktionen festgestellt werden. Innerhalb einer Kette wurden sogar mehrere Kurgane mit solchen zusätzlichen Befunden dokumentiert. Solche Ensembles befanden sich meistens im Zentrum des Gräberfeldes und zugleich im mittleren Bereich einer Kette. Peripheriekonstruktionen wurden meist im Umfeld der größten Kurgane des Gräberfeldes festgestellt. Die Lage solcher Kurgane innerhalb der Nekropole spielte dabei keine Rolle.

- Jede dritte Nekropole mit großen fürstlichen Kurganen der Saken wies sog. Prozessionswege auf. Solche „Straßen“ sind momentan nur im südöstlichen Siebenstromland bekannt. Breite und Durchmesser der sog. Prozessionswege sind unterschiedlich. Das Verhältnis von Kurgandurchmesser zum Durchmesser des sog. Prozessionsweges bleibt jedoch immer gleich, nämlich 3:5. Sowohl in einer Nekropole als auch in einer Kette traten mehrere Kurgane mit einem sog. Prozessionsweg auf. Bezüglich der Lage der Kurgane mit sog. Prozessionsweg konnte keine bestimmte Regel festgestellt werden.

- Auf jedem fünften Gräberfeld kamen Kurgane mit einem durchgehenden oder unterbrochenen Kreisgraben zum Vorschein. Solche Unterbrechungen konnten als „Eingänge“ in den sakralen Bereich eines Kurgans angesprochen werden. Sie befanden sich überwiegend in der Ost- bzw. Westhälfte der Anlage. Es konnten auf der Nekropole ein oder mehrere Kurgane mit einem Kreisgraben festgestellt werden. Es wurde kein konstantes Verhältnis von Kurgandurchmesser zum Durchmesser des Kreisgrabens festgestellt, was für das gesamte Untersuchungsgebiet zutraf. Innerhalb eines Gräberfeldes wurden allerdings bestimmte Proportionen beim Errichten der Kurgane mit Kreisgraben eingehalten.

- $\quad$ Um einige Kurgane herum wurden Steinkreise festgestellt. Solche Befunde kamen auf jedem dritten Gräberfeld zum Vorschein. Steinkreise wurden um Großkurgane herum errichtet. Sie fehlen jedoch in der Kategorie der sehr großen Kurgane komplett. Kurgane mit einem Steinkreis befanden sich in beiden Verbreitungszonen und waren auf den Gräberfeldern unregelmäßig verteilt. Ein bestimmtes Verhältnis zwischen Kurgandurchmesser und dem Durchmesser eines Steinkreises konnte nicht festgestellt werden. Steinkreise bildeten jedoch in allen Fällen die äußere Begrenzung der Kurgananlage.

- Zuletzt bleiben unter den Befunden in der Peripherie eines Kurgans noch die Sonderfälle zu erwähnen. Es handelt sich dabei um Erd- oder Steinwälle, doppelte Steinkreise, Plattformen oder Baurampen. Die Aussagekraft der Informationen zu diesen Bauelementen war wenig repräsentativ, da es sich um selten vorkommende Befunde handelte. 
- Fast alle Befunde im näheren Umfeld der Kurgane des südöstlichen Siebenstromlandes finden mehrere Parallelen in anderen Gebieten des skytho-sakischen Kulturkreises. Jedoch ist das Errichten der sog. Prozessionswege, der Steinwälle und der Baurampen eine Besonderheit des Forschungsgebietes.

- Möglicherweise dienten die sog. Prozessionswege, Kreisgräben, Steinkreise oder Wälle als eine Begrenzung des sakralen Raumes um den Grabhügel.

- Auf nur einem Drittel aller erforschten Gräberfelder befanden sich ausschließlich einfache, abgerundete Kurgane ohne weitere besondere Merkmale.

- Über die Hälfte aller Nekropolen wies Kurgane unterschiedlicher Form auf. Belegt sind abgerundete und viereckige Grabhügel. Die Peripherie der Kurgane kann frei von weiteren Befunden sein, oder in einigen Fällen von weiteren Befunden flankiert sein. Dabei treten in beiden Verbreitungszonen verschiedenste Kombination auf, die in den meisten Fällen allerdings einmalig sind.

- Ausschlaggebend für den Bau eines solchen Befundes in der Peripherie der Kurgane war wahrscheinlich die Größe des Kurgans. Diese Elemente in der Peripherie der Kurgane markieren bestimmte sakrale Bereiche und spiegeln eventuell zudem den sozialen Status des Bestatteten wider.

- Die Bedeutung jedes einzelnen Elementes in der Peripherie sowie Fragen zur Kombination unterschiedlicher Befunde um einen Kurgan herum konnten anhand der Untersuchungen an der Oberfläche nicht geklärt werden. Hierzu sind zukünftige Untersuchungen notwendig, die natürlich zunächst mit dem vollständigen Ausgraben der Kurgane verbunden sind.

- Es gibt einige wenige früheisenzeitliche Kurgane im südöstlichen Siebenstromland, die komplett oder zum Teil ausgegraben sind.

- Kein einziges Gräberfeld wurde vollständig erforscht.

- Die untersuchten Großkurgane wiesen in ihrer Aufschüttung drei bis vier Schichten auf, die abwechselnd aus einer Schicht lehmigen Sandes oder Kieselsandes und einer Steinpackung bestanden. Im Zentrum der Kurgane baute man eine Halbkugel aus Lehm. Diese Halbkugel wurde mit Lehmmörtel verschmiert und anschließend mit einer Steinpackung abgedeckt. Die darauf folgenden Schichten aus Steinen, gestampftem Lehm oder lehmigem Sand verliehen der Konstruktion eine plattformähnliche bzw. pyramidenähnliche Form mit abgeflachter Kuppe. Drei steile und ein sanfter Abhang wurden bereits in dieser Bauphase angelegt. Als letzte Schicht folgte eine Steinpackung, die entweder die komplette Oberfläche oder nur die untere Hälfte des Kurgans bedeckte.

- In einigen Fällen wurden gestampfte Lehmziegel zur Errichtung des Kurgans verwendet. Sie wurden durch Lehmmörtel miteinander verbunden.

- Meist im Zentrum der Kurgane kam eine westöstlich ausgerichtete, rechteckige Grabgrube mit abgerundeten Ecken oder eine quadratische Grabgrube mit einer Einzelbestattung eines erwachsenen Individuums zutage.

- $\quad$ Die Bestatteten ruhten in Rückenstreckerlage mit dem Kopf nach Westen oder Südwesten.

- Zum Beigabenrepertoire gehörten Waffen, wie Dolche / Akinakai und Schwerter, Trachtgegenstände und Schmuck. Außerdem wurden Gefäße aus verschiedenen Materialien wie Ton, Bronze und Silber sowie Silberlöffel, Holzkellen und -tabletts, beigegeben.

- Die Gräberfelder der ersten Verbreitungszone befanden sich ausnahmslos in Bezug zu fließenden Gewässern, überwiegend am linken westlichen Flussufer.

- In der zweiten Verbreitungszone wurden die Gräberfelder auf dem rechten Ufer oder zwischen zwei fließenden Gewässern angelegt. Dabei spielte die Flussseite offensichtlich eine weniger wich- 
tige Rolle als bei den Gräberfeldern der ersten Verbreitungszone. Außerdem ist die Lage von Nekropolen auf Riedelkanten unweit von Flüssen oder in Bergtälern belegt.

- Flüsse und Bäche stellten möglicherweise eine natürliche Grenze zwischen den sakralen Bereichen der jeweiligen Nekropolen dar.

- Nach der Analyse der äußeren Merkmale der Kurgane im Zusammenhang mit den Beschreibungen Herodots wurde, unter Vorbehalt, festgestellt, dass die Kurgane der sakischen Elite den skythischen Heiligtümern des Ares ähneln. Gräberfelder mit Kurganen dieser besonderen Art stellen wahrscheinlich nicht nur Bestattungsplätze im eigentlichen Sinne dar, sondern Orte des kollektiven Gedächtnisses und der Selbstidentifikation der Saken. 